\title{
LETTING GO \\ OF THE DICHOTOMY BETWEEN PSORIASIS \\ AND PSORIATIC ARTHRITIS
}

\section{EMMERIKLEUJTEN}





\section{Letting go of the dichotomy between psoriasis and psoriatic arthritis}

Emmerik F.A. Leijten 
ISBN: $\quad$ 978-94-6416-619-4

Cover design: Publiss | www.publiss.nl

Lay-out \& Print: Ridderprint | www.ridderprint.nl

(c) Copyright 2021: Emmerik F.A. Leijten, Utrecht, The Netherlands All rights reserved. No part of this publication may be reproduced, stored in a retrieval system, or transmitted in any form or by any means, electronic, mechanical, by photocopying, recording, or otherwise, without the prior written permission of the author.

Printing of this thesis was partially financially supported by Infection \& Immunity Utrecht. 


\title{
Letting go of the dichotomy between psoriasis and psoriatic arthritis
}

\section{Psoriasis en artritis psoriatica: voorbij de dichotomie}

(met een samenvatting in het Nederlands)

\section{Proefschrift}

\author{
ter verkrijging van de graad van doctor aan de \\ Universiteit Utrecht \\ op gezag van de \\ rector magnificus, prof.dr. H.R.B.M. Kummeling, \\ ingevolge het besluit van het college voor promoties \\ in het openbaar te verdedigen op
}

dinsdag 29 juni 2021 des middags te 4.15 uur

door

Emmerik Frederick Adriaan Leijten

geboren op 19 januari 1984

te Miami, Verenigde Staten 


\section{Promotoren:}

Prof. dr. T.R.D.J. Radstake

Dr. M.L. Boes 


\section{Content}

$\begin{array}{lll}\text { CHAPTER } 1 \text { General introduction } & 7\end{array}$

CHAPTER 2 Screening for psoriatic arthritis using the Psoriasis 21

Epidemiology Screening Tool questionnaire:

examining the optimal cut-off

CHAPTER 3 First-line csDMARD monotherapy drug retention

in psoriatic arthritis: methotrexate outperforms

sulfasalazine

CHAPTER 4 Enrichment of Activated Group 3 Innate Lymphoid Cells in

Psoriatic Arthritis Synovial Fluid

CHAPTER 5 Innate lymphoid cells: sparking inflammatory rheumatic

disease?

CHAPTER 6 Tissue-resident memory CD8+ T cells from skin

differentiate psoriatic arthritis from psoriasis

CHAPTER 7 Broad proteomic screen reveals shared serum proteomic

signature in patients with psoriatic arthritis and psoriasis without arthritis

CHAPTER 8 Limits of traditional evidence-based medicine methodologies exemplified by the novel era in psoriatic arthritis drug development

CHAPTER 9 General Discussion

List of publications

Summary in Dutch (Nederlandse vertaling)

Acknowledgements (Dankwoord)

Curriculum vitae 


$$
1
$$




\section{CHAPTER 1}

General introduction 
In the introduction to this thesis we first provide the reader with an overview of the clinical features of psoriasis and psoriatic arthritis (PsA). Next, a historical approach is taken to clarify to the readers why PsA is deemed fitting within the umbrella of spondyloarthritis-type diseases, which provides the starting point of our current concept of the immunopathology of PSA. Lastly, we discuss caveats in our clinical and immunological knowledge that are tackled in each of the chapters of the thesis.

\section{Psoriasis and psoriatic arthritis}

Why do some patients with psoriasis develop psoriatic arthritis? This seemingly simple question, concerning the distinction between psoriasis and PsA is the main theme of this thesis. When teaching about PsA to medical students, many rheumatologists might define PsA as the coincidence of psoriasis with inflammatory musculoskeletal disease, in which the musculoskeletal disease is less likely to be explained by another cause. This simple " $1+1=2$ " concept stooled on clinical reasoning provides a solid starting point to be kept in mind, even if there are exceptions to the rule. There are well-established classification criteria available for PsA which aid in defining homogenous patient populations for the sake of research purposes, but it is important to reiterate that classification criteria are not used for making a clinical diagnosis of PsA(1).

Psoriasis is a relatively common auto-immune disease of the skin, with a prevalence of approximately two percent of the global population(2). From the larger pool of patients affected by psoriasis, a minority will develop inflammatory musculoskeletal disease that can clinically be diagnosed as PsA by the treating physician. It is unknown why some patients with psoriasis will develop PsA, whereas other patients with psoriasis will never develop musculoskeletal manifestations. In the general population, the prevalence of PsA is estimated at $0.02 \%$ to $0.25 \%$ (3). In patients with psoriasis, the cited prevalence of PsA depends on the study with lower estimates at 5\% and higher estimates at 40\%(4-9). The large variation in prevalence reported is partly explained by the clinical heterogeneity of the disease, further specified in the next section.

\section{Psoriatic disease is clinically heterogeneous}

Both psoriasis and PsA are clinically heterogeneous. Psoriasis typically manifests as scaly, itchy, red, raised plaques located on extensor surfaces of the body. Psoriasis can present in different forms and the most common form is plaque psoriasis (psoriasis vulgaris). Other forms include guttate psoriasis (coin-shaped lesions) and pustular psoriasis (pustules typically found on the soles of feet and palms of hands). A single patient can present with multiple forms of psoriasis. 
The severity of the disease shows considerable variation: from a minor skin lesion that is barely noticed by the patient, to very large areas covering many parts of the body surface. In terms of the anatomic location affected, the extensor surfaces of elbows and knees are prototypical sites. Other common anatomical sites affected include the scalp ("psoriasis capitis"), the groin ("inverse psoriasis"), genital region, between the buttocks, in the belly button, and the inner ears.

A typical manifestation of psoriasis is the presence of psoriatic nail changes, occurring in roughly half of the patients, which manifests as nail pitting, onycholysis, subungual hyperkeratosis and/or the oil drop sign(2).

The musculoskeletal manifestations of PsA are similarly heterogeneous. The most typical manifestation is arthritis in a patient with known history of psoriasis. On average, PsA is diagnosed 10 years after the first onset of psoriasis $(9,10)$. However, sometimes PsA is diagnosed in the absence of psoriasis. These patients are diagnosed with "PsA sine psoriasis" and may or may not develop psoriasis later on in life(10). Some clinical features have been associated with an increased risk of developing PSA in the larger pool of psoriasis patients. The presence of psoriatic nail changes in patients with psoriasis is a risk factor for developing arthritis in many studies $(9,10)$. Some studies have found that the anatomical site affected by psoriasis (e.g. of the scalp) and greater severity of psoriasis could be risk factors for developing PsA $(9,11)$. Uncovering these risk factors contributes to our understanding of the pathophysiology of PsA, but do not directly translate into clinically meaningful tools to monitor subpopulations within the larger pool of psoriasis patients(12). For example, patients with very mild psoriatic skin disease can develop severe joint disease and most patients with severe psoriasis will never develop musculoskeletal manifestations in their lifetime(12). To summarize, there is no strong clinical correlate between the severity of cutaneous manifestations and the presence or severity of musculoskeletal manifestations.

Other musculoskeletal manifestations of PsA including dactylitis ("sausage digit"), enthesitis and sacroiliitis/spondylitis. Inflammatory bowel disease and anterior uveitis are extra-articular manifestations much more commonly seen in patients with psoriasis or PsA as compared to the general population(13). 
Figure 1 illustrates the clinical heterogeneity of PsA. Most patients with psoriasis do not have PsA (A). The classic presentation of PsA would be concomitant psoriasis and arthritis (B). However, in the absence of the classic presentation there are other combinations of clinical manifestations that can sometimes lead the treating physician to diagnose PsA, even in the absence of psoriasis (C-D)(10,14).

A

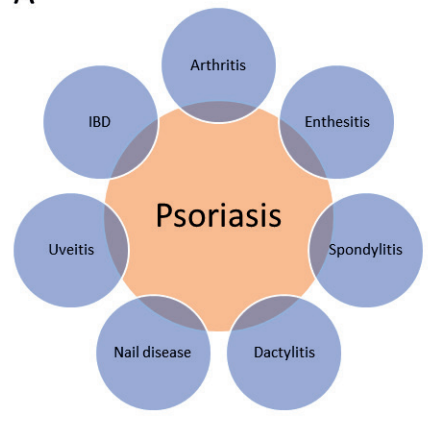

C

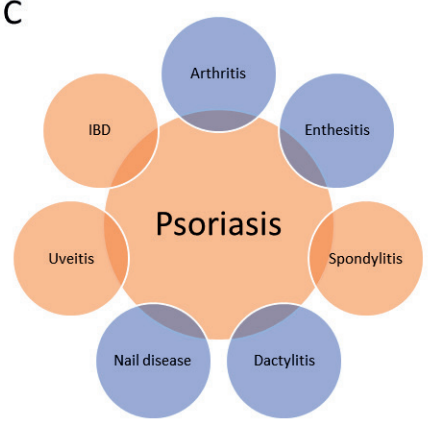

B
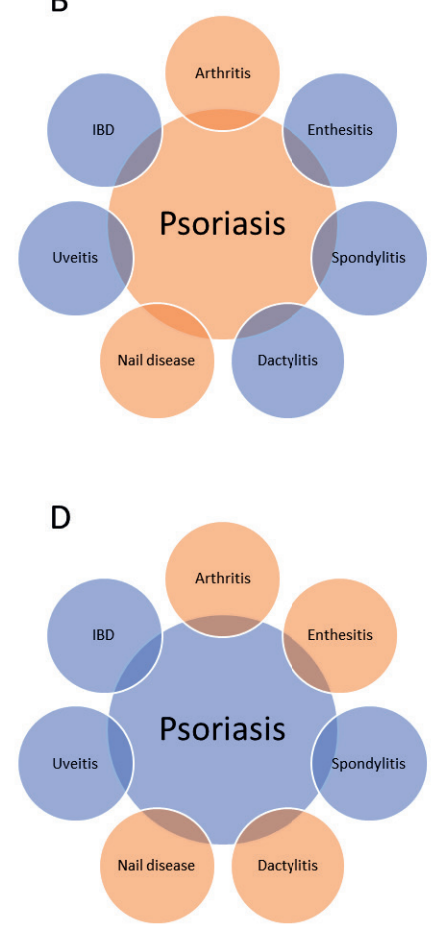

Figure 1. Clinical features of psoriasis and psoriatic arthritis. Some examples of different clinical phenotypes are presented: psoriasis only (A), "classic" psoriatic arthritis (B), psoriasis with axial disease and extra-articular manifestations (C), and psoriatic arthritis sine psoriasis (D). Spondylitis refers to the so-called axial disease presentation with sacro-iliitis. Uveitis refers to anterior uveitis. Abbreviations: IBD = inflammatory bowel disease.

In order to help understand why these seemingly heterogeneous features are often viewed in context of underlying psoriasis / PsA, we will now provide background on the concept of spondyloarthritis. A selection of historical timepoints is taken to clarify the prevailing clinical and pathophysiological perspective of PSA at the onset of this thesis. 


\section{Historical overview}

\section{4: Psoriasis associated with arthritis}

In 1934 the sixth edition of The Merck Manual was published containing a dedicated section on psoriasis and a dedicated section on arthritis(15). The prevalence and clinical description of psoriasis presented in this work remains conserved to this day. The treatment options mentioned in the book include sea voyages and ultraviolet light therapy, the latter still a viable treatment option today. Specific lifestyle modifications were also recommended at the time, including a lowprotein or vegetarian diet and avoidance of alcohol, coffee and other stimulants.

In the 1934 edition the term psoriatic arthritis was never mentioned. Nonetheless, in the section on psoriasis it is stated that patients with psoriasis can have "rheumatic or gouty tendencies". Furthermore, the section on Arthritis Deformans lists under the potential symptoms that "psoriasis may develop". Other historical accounts at the end of the $19^{\text {th }}$ century have similarly described the association between psoriasis and arthritis(16-18). In summary, at the time the association between psoriasis and arthritis was noted, but PsA was not considered a separate entity.

\section{3: PsA distinct from RA}

In 1973, Professor Verna Wright and colleagues published a comprehensive characterization of PsA using data from several distinct, large cohorts (17-20). At that time, the argument was made to stop considering PsA as a variant of rheumatoid arthritis (RA). Instead of "lumping" PsA with RA, PsA was proposed to be part of the so-called "seronegative spondarthritides". This reasoning was based on a number of observations in these patients, including: (i) the absence of rheumatoid factor ("seronegativity"), (ii) the absence of subcutaneous (rheumatoid) nodules, (iii) the increased prevalence of sacro-iliitis, (iv) the clear familial aggregation of "seronegative spondarthritides" and (v) an overlap in clinical features that includes psoriatic skin or nail disease, uveitis and colitis(19).

This clinical reasoning is retrospectively a core starting point for the distinction between RA and PsA, falling within spondyloarthritis group $(17,20)$.

\section{4: PsA treated the same as RA}

In 2004, the Dutch reference textbook for medical students defined PsA as the coincidence of rheumatoid-factor negative arthritis with psoriasis(21). The initial distinction between PsA and RA argued by Verna Wright and others continued to be corroborated: PsA affects a different clinical pattern than seen in RA. The presence of a sausage digit, or dactylitis, was another feature specifically attributed to PsA. 
It was also clear that PSA shared substantial clinical and pathogenic overlap with ankylosing spondylitis, including the propensity to develop sacroiliitis and increased frequency of HLA-B27 allele in patients with PSA(14). Data also confirm the strong familial clustering, with an estimated 40 -fold increase in the development of PSA when a first-degree family member has PsA(22,23).

Histological studies provided additional support for the distinction between PsA and RA. In PSA more prominent changes were noted in arterioles, capillaries and endothelial cells at the synovial tissue(24-28). Another histological features attributed to PSA was the presence of tertiary lymphoid structures and analysis of T cells in the synovial fluid showed that CD8 T cells were more frequent in PsA as compared to RA(29,30).

Despite the well-accepted distinction between PSA and RA diseases/pathology, patients with PsA patients were recommended to follow the exact same treatment strategy as in RA, including conventional disease-modifying anti-rheumatic drugs (CDMARDs) such as methotrexate. Similar to RA, Tumour necrosis factor-a inhibitors were also proven effective in treating psoriasis and $\operatorname{PsA}(26,31)$. Only in the following years would specific treatment options become available that were efficacious in psoriasis/PsA but not RA.

\section{2: Specifying immunopathology in spondyloarthritis}

In 2012, a seminal publication by Sherlock et. al described how overproduction of IL-23 in mice led to clinical features that closely resemble the manifestations of spondyloarthritis in humans(32). Specific parts of this model are worth mentioning. Firstly, in this animal model inflammation of the enthesis-the site where tendon or ligament attaches to bone-occurred prior to the development of arthritis, suggesting that "enthesitis" could be an initial triggering event. Secondly, the model was driven by interleukin (IL)-23-responsive T cells residing in the enthesis, which produced effector molecules such as IL-17 and IL-22. The specific type of $T$ cells in this animal model was not yet clarified at that time point. The overall message was clear: IL-23/IL-17 axis was deemed an important molecular pathway in the initiation of spondyloarthritis. The results from this animal model were in line with the hypothesis previously put forward by McGonagle and colleagues: an inflammatory process in enthesis could help explain the spectrum of clinical phenotypes seen in the overarching group of spondyloarthritis(33-37).

The proof of the pudding-so to speak-was that the cytokines in this pathway could be targeted by monoclonal antibodies and eventually proved efficacious for psoriasis and PsA in clinical trials $(2,38)$. 


\section{Thesis outline}

So far we have described the clinical heterogeneity of psoriasis and PsA. We summarized how advances in basic scientific studies helped bring new therapeutic targets to the market for PsA, which in turn substantiated the clinical reasoning reported in the 1970s. Regardless of these advances, there remain specific clinical and immunologic questions unanswered at the start of this thesis.

\section{Clinical section:}

Both psoriasis and PsA can have a negative impact on quality of life. For psoriasis, this is partly dependent on the amount of body surface area affected and on the anatomical location it affects. For example, more visible areas could impact the quality of life for patients with specific professions. Also, psoriasis affecting the genital region can negatively impact sexuality and quality of life $(39,40)$. PsA can also negatively impact the quality of life, independent of psoriasis(41). Considering the fact that the minority of psoriasis patients will develop PsA, there is a need for practical means by which patients with PsA can be identified. Furthermore, many patients with PsA have irreversible joint damage at first diagnosis(42).

Screening for PsA also allows treatment aimed at musculoskeletal manifestations to be optimized, with the ultimate goal of reducing burden of the disease and preventing irreversible joint damage. In Chapter $\mathbf{2}$ we first determine the prevalence of PsA in psoriasis patients being seen by the dermatologist in our academic medical centre. We next examine whether a proposed screening method-a simple questionnaire to be used by the treating dermatologist-can accurately identify psoriasis patients with and without PsA and serve as a tool to guide which patients should be referred to a rheumatologist(42-44).

In terms of treatment, patients with PsA are often treated for long durations of time and it is not uncommon for multiple drugs to be prescribed-either sequentially or in combination-before a satisfactory balance between efficacy and side-effects is achieved. As mentioned in the historical section, the treatment regimen for PsA has by some been considered a "copy and paste" of RA. The clearest example of this is the use of methotrexate, the cornerstone of treatment in RA, while methotrexate lacks high level evidence studies supporting its use in the treatment in PsA. In Chapter 3 we therefore set out to describe which cDMARDs are used as first-line treatment in patients with PSA in daily clinical practice. We describe and compare the monotherapy drug retention rates of cDMARDs, as a surrogate outcome for treatment efficacy in daily clinical practice. Other factors that negatively impact drug-retention are explored, including side-effects leading to treatment cessation. 


\section{Immunologic section:}

It is unknown whether psoriasis and PsA are distinct diseases or rather fall within a single spectrum of disease(45-47). Why is this so relevant? One important driving factor for uncovering the molecular mechanism unique to PsA is the fact that most therapeutic agents are much more effective at halting psoriasis than they are at halting arthritis $(9,38,48)$. Another important reason to better define the immunological features found in PsA as compared to psoriasis: psoriasis is a strong risk factor for developing rheumatic disease and defining immunological factors that induce or protect from the development of rheumatic disease could bring to light more broadly applicable therapeutic targets. In brief, there is progress to be made at uncovering the specific immunopathology driving PsA which could indirectly provide insight into other immune-mediated rheumatic diseases.

In Chapter $\mathbf{4}$ and Chapter $\mathbf{5}$ we examine the specific role for innate lymphoid cells (ILCS) in the pathogenesis of PSA. This was a hypothesis-driven investigation principally driven (i) by the knowledge that resident immune cells at the enthesis could initiate spondyloarthritis in an animal models of spondyloarthritis (highlighted in the historical section) and (ii) by emerging studies that ILCs were detected in the skin of psoriasis patients with a role in the IL-23/IL-17 axis, without requiring an adaptive immune response $(49,50)$.

In Chapter $\mathbf{6}$ and Chapter $\mathbf{7}$ we take a "less" hypothesis-driven investigation: we compare a broad array of immune cell subsets and inflammatory mediators in the circulation of patients with psoriasis in whom PsA was excluded as compared to patients with PsA. Surprisingly, immunological studies that compare these two groups in a "head-to-head" manner are scarcely reported in the literature yet necessary to understand PsA development in psoriasis(51-54). The objective is to specify immunologic features unique to PsA. The methodological approach in these chapters is to keep the two groups as comparable as possible (e.g. similar level of psoriatic skin disease severity) and study patients not being treated with immunomodulatory drugs that may confound laboratory investigations.

\section{Future directions:}

Lastly, in Chapter $\mathbf{8}$ we reflect upon the immunological heterogeneity of PSA in the context of the rapidly increasing number of therapeutic targets that have already entered the market or will enter the market for PsA $(38,48)$. The celebrated progress in the field indirectly creates challenges for future trial design, making it even more difficult to translate data from clinical trials into daily clinical practice if we adhere to conventional standards. 
In summary, the main questions addressed in the current thesis are:

- "How can we best screen for PsA and how well do first-line cDMARDs work in daily clinical practice?" (Chapters 2-3)

- "Can we identify specific immunological factors that drive the development of PsA in patients with psoriasis?" (Chapters 4-7)

- "What are the major challenges and potential avenues of future trial design to move the field forward?" (Chapter 8) 


\section{References}

1. Taylor W, Gladman D, Helliwell P, Marchesoni A, Mease P, Mielants H. Classification criteria for psoriatic arthritis: development of new criteria from a large international study. Arthritis Rheum. 2006 Aug;54(8):2665-73.

2. Greb JE, Goldminz AM, Elder JT, Lebwohl MG, Gladman DD, Wu J, et al. Psoriasis. Nat Rev Dis Prim. 2016 Dec 22;2(1):16082.

3. Setty AR, Choi HK. Psoriatic arthritis epidemiology. Curr Rheumatol Rep. 2007 Dec 7;9(6):449-54.

4. Alinaghi F, Calov M, Kristensen LE, Gladman DD, Coates LC, Jullien D, et al. Prevalence of psoriatic arthritis in patients with psoriasis: A systematic review and meta-analysis of observational and clinical studies. J Am Acad Dermatol. 2019 Jan;80(1):251-265.e19.

5. Mease PJ, Gladman DD, Papp KA, Khraishi MM, Thaçi D, Behrens F, et al. Prevalence of rheumatologist-diagnosed psoriatic arthritis in patients with psoriasis in European/North American dermatology clinics. J Am Acad Dermatol. 2013 Nov;69(5):729-35.

6. Gelfand JM, Gladman DD, Mease PJ, Smith N, Margolis DJ, Nijsten T, et al. Epidemiology of psoriatic arthritis in the population of the United States. J Am Acad Dermatol. 2005 Oct;53(4):573.e1-573.e13.

7. Karreman MC, Weel AEAM, van der Ven M, Vis M, Tchetverikov I, Nijsten TEC, et al. Prevalence of Psoriatic Arthritis in Primary Care Patients With Psoriasis. Arthritis Rheumatol. 2016 Apr;68(4):924-31.

8. Ogdie A, Weiss P. The Epidemiology of Psoriatic Arthritis. Rheum Dis Clin North Am. 2015 Nov;41(4):545-68.

9. Scher JU, Ogdie A, Merola JF, Ritchlin C. Preventing psoriatic arthritis: focusing on patients with psoriasis at increased risk of transition. Nat Rev Rheumatol. 2019 Mar 11;15(3):153-66.

10. Ritchlin CT, Colbert RA, Gladman DD. Psoriatic Arthritis. N Engl J Med. 2017 Mar 9;376(10):957-70.

11. Eder L, Haddad A, Rosen CF, Lee K-A, Chandran V, Cook R, et al. The Incidence and Risk Factors for Psoriatic Arthritis in Patients With Psoriasis: A Prospective Cohort Study. Arthritis Rheumatol. 2016 Apr;68(4):915-23.

12. Wilson FC, Icen M, Crowson CS, McEvoy MT, Gabriel SE, Kremers HM. Incidence and clinical predictors of psoriatic arthritis in patients with psoriasis: A population-based study. Arthritis Rheum. 2009 Feb 15;61(2):233-9.

13. Turk M, Hayworth J, Nevskaya T, Pope J. The frequency of uveitis in patients with adult versus childhood spondyloarthritis. RMD Open. 2020 Aug 31;6(2):e001196.

14. Feld J, Chandran V, Haroon N, Inman R, Gladman D. Axial disease in psoriatic arthritis and ankylosing spondylitis: a critical comparison. Nat Rev Rheumatol. 2018 Jun 11;14(6):363-71.

15. The Merck Manual. Sixth Edit. Rahway, N.J.: Merck \& CO. Inc.; 1934.

16. The Lancet. Psoriatic arthritis: classification and holistic management. Lancet. 2018 Jun;391(10136):2185.

17. Helliwell PS. Verna Wright: father of the spondyloarthropathies. Ann Rheum Dis. 2019;78(4): 454-5. 
18. Espinoza LR. The History of Psoriatic Arthritis (PsA): From Moll and Wright to Pathway-Specific Therapy. Curr Rheumatol Rep. 2018 Oct 9;20(10):58.

19. Moll JMH, Wright V. Psoriatic arthritis. Semin Arthritis Rheum. 1973 Jan;3(1):55-78.

20. Gladman DD. Psoriatic Arthritis from Wright's Era Until Today. J Rheumatol Suppl. 2009 Aug $1 ; 83: 4-8$.

21. J.W.J. Bijlsma, P.P.M.M. Geusens, C.G.M. Kallenberg, P.P. Tak JWGJ, editor. Reumatologie en klinische immunologie. Houten: Bohn Stafleu van Loghum; 2004.

22. Chandran V, Schentag CT, Brockbank JE, Pellett FJ, Shanmugarajah S, Toloza SMA, et al. Familial aggregation of psoriatic arthritis. Ann Rheum Dis. 2009 May;68(5):664-7.

23. Karason A, Love TJ, Gudbjornsson B. A strong heritability of psoriatic arthritis over four generations--the Reykjavik Psoriatic Arthritis Study. Rheumatology. 2009 Nov 1;48(11):1424-8.

24. Kruithof E, Baeten D, De Rycke L, Vandooren B, Foell D, Roth J, et al. Synovial histopathology of psoriatic arthritis, both oligo- and polyarticular, resembles spondyloarthropathy more than it does rheumatoid arthritis. Arthritis Res Ther. 2005 Jan;7(3):R569-80.

25. Espinoza LR, Vasey FB, Espinoza CG, Bocanegra TS, Germain BF. Vascular changes in psoriatic synovium. a light and electron microscopic study. Arthritis Rheum. 1982 Jun;25(6):677-84.

26. van Kuijk AWR, Reinders-Blankert P, Smeets TJM, Dijkmans BAC, Tak PP. Detailed analysis of the cell infiltrate and the expression of mediators of synovial inflammation and joint destruction in the synovium of patients with psoriatic arthritis: implications for treatment. Ann Rheum Dis. 2006 May 25;65(12):1551-7.

27. Baeten D, Demetter P, Cuvelier C, Van den Bosch F, Kruithof E, Van Damme N, Verbruggen G, Mielants H, Veys EM, De Keyser EF. Comparative study of the synovial histology in rheumatoid arthritis, spondyloarthropathy, and osteoarthritis: influence of disease duration and activity. Ann Rheum Dis. 2000 Dec 1;59(12):945-53.

28. Reece RJ, Canete JD, Parsons WJ, Emery P, Veale DJ. Distinct vascular patterns of early synovitis in psoriatic, reactive, and rheumatoid arthritis. Arthritis Rheum. 1999 Jul;42(7):1481-4.

29. Canete JD, Santiago B, Cantaert T, Sanmarti R, Palacin A, Celis R, et al. Ectopic lymphoid neogenesis in psoriatic arthritis. Ann Rheum Dis. 2007 Jan 12;66(6):720-6.

30. Costello P, Bresnihan B, O'Farrelly C, FitzGerald O. Predominance of CD8+ T lymphocytes in psoriatic arthritis. J Rheumatol. 1999 May;26(5):1117-24.

31. Mease PJ, Goffe BS, Metz J, VanderStoep A, Finck B, Burge DJ. Etanercept in the treatment of psoriatic arthritis and psoriasis: a randomised trial. Lancet. 2000 Jul;356(9227):385-90.

32. Sherlock JP, Joyce-Shaikh B, Turner SP, Chao C-C, Sathe M, Grein J, et al. IL-23 induces spondyloarthropathy by acting on ROR-yt+ CD3+CD4-CD8- entheseal resident $\mathrm{T}$ cells. Nat Med. 2012 Jul;18(7):1069-76.

33. McGonagle D, Marzo-Ortega H, O'Connor P, Gibbon W, Hawkey P, Henshaw K EP. Histological assessment of the early enthesitis lesion in spondyloarthropathy. Ann Rheum Dis. 2002 Jun 1;61(6):534-7.

34. McGonagle D, Gibbon W, Emery P. Classification of inflammatory arthritis by enthesitis. Lancet. 1998 Oct;352(9134):1137-40. 


\section{CHAPTER 1}

35. McGonagle D, Aydin SZ, Gül A, Mahr A, Direskeneli H. 'MHC-I-opathy'-unified concept for spondyloarthritis and Behçet disease. Nat Rev Rheumatol. 2015;11(12):731-40.

36. Blauvelt A, Chiricozzi A. The Immunologic Role of IL-17 in Psoriasis and Psoriatic Arthritis Pathogenesis. Clin Rev Allergy Immunol. 2018 Dec 14;55(3):379-90.

37. Lubberts E. The IL-23-IL-17 axis in inflammatory arthritis. Nat Rev Rheumatol. 2015 Jul 28;11(7):415-29.

38. Baker KF, Isaacs JD. Novel therapies for immune-mediated inflammatory diseases: What can we learn from their use in rheumatoid arthritis, spondyloarthritis, systemic lupus erythematosus, psoriasis, Crohn's disease and ulcerative colitis? Ann Rheum Dis. 2018 Feb;77(2):175-87.

39. da Silva N, Augustin M, Langenbruch A, Mrowietz U, Reich K, Thaçi D, et al. Sex-related impairment and patient needs/benefits in anogenital psoriasis: Difficult-to-communicate topics and their impact on patient-centred care. Zirlik A, editor. PLoS One. 2020 Jul 1;15(7):e0235091.

40. da Silva N, Augustin M, Langenbruch A, Mrowietz U, Reich K, Thaçi D, et al. Disease burden and treatment needs of patients with psoriasis in sexually-sensitive and visible body areas: results from a large-scale survey in routine care. Eur J Dermatology. 2020 Jun;30(3):267-78.

41. Rosen CF, Mussani F, Chandran V, Eder L, Thavaneswaran A, Gladman DD. Patients with psoriatic arthritis have worse quality of life than those with psoriasis alone. Rheumatology. 2012 Mar 1;51(3):571-6.

42. Haroon M, Gallagher P, FitzGerald O. Diagnostic delay of more than 6 months contributes to poor radiographic and functional outcome in psoriatic arthritis. Ann Rheum Dis. 2015 Jun;74(6):1045-50.

43. Gladman DD, Thavaneswaran A, Chandran V, Cook RJ. Do patients with psoriatic arthritis who present early fare better than those presenting later in the disease? Ann Rheum Dis. 2011 Dec 1;70(12):2152-4.

44. Kane D, Stafford L, Bresnihan B, FitzGerald O. A prospective, clinical and radiological study of early psoriatic arthritis: an early synovitis clinic experience. Rheumatology (Oxford). 2003 Dec;42(12):1460-8.

45. Sakkas LI, Bogdanos DP. Are psoriasis and psoriatic arthritis the same disease? The IL-23/IL17 axis data. Autoimmun Rev. 2017 Jan;16(1):10-5.

46. Chimenti MS, Caso F, Alivernini S, De Martino E, Costa L, Tolusso B, et al. Amplifying the concept of psoriatic arthritis: The role of autoimmunity in systemic psoriatic disease. Autoimmun Rev. 2019 Jun; 18(6):565-75.

47. Boehncke W-H. Psoriasis and Psoriatic Arthritis: Flip Sides of the Coin? Acta Derm Venereol. 2016 May;96(4):436-41.

48. Furue $K$, Ito $T$, Furue M. Differential efficacy of biologic treatments targeting the TNF-a/IL-23/ IL-17 axis in psoriasis and psoriatic arthritis. Cytokine. 2018 Aug;111:182-8.

49. Villanova F, Flutter B, Tosi I, Grys K, Sreeneebus H, Perera GK, et al. Characterization of innate lymphoid cells in human skin and blood demonstrates increase of NKp44+ ILC3 in psoriasis. J Invest Dermatol. 2014 Apr;134(4):984-91. 
50. Sherlock JP, Joyce-Shaikh B, Turner SP, Chao C-C, Sathe M, Grein J, et al. IL-23 induces spondyloarthropathy by acting on ROR-yt+ CD3+CD4-CD8- entheseal resident $\mathrm{T}$ cells. Nat Med. 2012 Jul; 18(7):1069-76.

51. Pollock RA, Abji F, Liang K, Chandran V, Pellett FJ, Virtanen C, et al. Gene expression differences between psoriasis patients with and without inflammatory arthritis. J Invest Dermatol. 2015 Feb;135(2):620-3.

52. Abji F, Pollock RA, Liang K, Chandran V, Gladman DD. Brief Report: CXCL10 Is a Possible Biomarker for the Development of Psoriatic Arthritis Among Patients With Psoriasis. Arthritis Rheumatol (Hoboken, NJ). 2016;68(12):2911-6.

53. Benham H, Norris P, Goodall J, Wechalekar MD, Fitzgerald O, Szentpetery A, et al. Th17 and Th22 cells in psoriatic arthritis and psoriasis. Arthritis Res Ther. 2013 Jan;15(5):R136.

54. Chandran V, Cook RJ, Edwin J, Shen H, Pellett FJ, Shanmugarajah S, et al. Soluble biomarkers differentiate patients with psoriatic arthritis from those with psoriasis without arthritis. Rheumatology. 2010;49(7):1399-405. 


$$
2
$$




\section{CHAPTER 2}

\section{Screening for psoriatic arthritis using the Psoriasis Epidemiology Screening Tool questionnaire: examining the optimal cut-off}

Authors:

E.F.A. Leijten ${ }^{1}$, V. Sigurdsson², M.H. Wenink ${ }^{3}$, T.R.D.J. Radstake

Affiliations:

1. Department of Rheumatology and Clinical Immunology, UMC Utrecht, the Netherlands

2. Department of Dermatology, UMC Utrecht, the Netherlands 3. Sint Maartenskliniek, Department of Rheumatology, Nijmegen, the Netherlands

Published in:

British Journal of Dermatology 2017 May; 176(5):1357-1359

doi: $10.1111 /$ bjd. 14953 
Dear Editor, we read the recently published article in this journal by Coates et al. with great interest.(1) Indeed, early detection of psoriatic arthritis (PsA) in patients with psoriasis is necessary to adequately treat concomitant joint disease, which can significantly impact quality of life. $(2,3)$ The Psoriasis Epidemiology Screening Tool (PEST) is a short screening questionnaire that could guide dermatologist decision making, $(4,5)$ and Coates et al. have provided evidence that the PEST may be used in both the primary and secondary care settings. $(1,6)$ One key finding is that they have questioned the original PEST cut-off score that would warrant rheumatologist referral, with their data now suggesting that a lower cut-off point may be more appropriate.(1) Before recommendations can be made on the implementation of alternative cut-off values for the PEST as diagnostic screening tool, it is essential to verify whether this is equally valid and performs similarly in different clinical settings in different places worldwide.

We evaluated the diagnostic efficacy of the PEST questionnaire in patients attending a psoriasis clinic in an academic teaching hospital in the Netherlands, where a translated version of the PEST was routinely administered. As part of a collaborative effort the rheumatologists and dermatologists ran a parallel clinic: all patients with psoriasis - regardless of their PEST score - were invited for a separate clinical evaluation consisting of a thorough medical history, physical examination and, when necessary, laboratory and radiological studies to confirm or exclude PsA. The medical ethical committee approved the study and records from January 2014 to April 2016 were reviewed. The sensitivity, specificity, positive predictive value and negative predictive value were calculated based on the number of subjects meeting CASPAR (Classification criteria for Psoriatic Arthritis) criteria.(7) As a cut-off for a positive or negative PEST score, we analyzed both the originally recommended score of $\geq 3$, as well as the newly proposed score of $\geq 2$. We used the methods by DeLong et al. (using MedCalc version 16.4.3; MedCalc, Ostend, Belgium)(8) to calculate and compare area under the receiver-operator curves (AUC).

Overall, the PEST questionnaire was performed in 134 patients with psoriasis. Thirty-seven patients did not wish to have an evaluation by a rheumatologist, and there were a further 11 in whom a rheumatologist had previously established a diagnosis of PsA (Fig. 1). In the remaining 86 patients, 18 new cases of PsA were identified, yielding a prevalence of $21 \%$ for newly diagnosed PsA. The overall prevalence of PSA was $30 \%(n=29 / 97)$ based on all patients who underwent a clinical evaluation, while the overall prevalence of PSA dropped to a minimum of $22 \%$ ( $n=29 / 134$ ) if we also included the patients that did not undergo clinical evaluation and we assumed none of them had PsA. 
Figure 1.

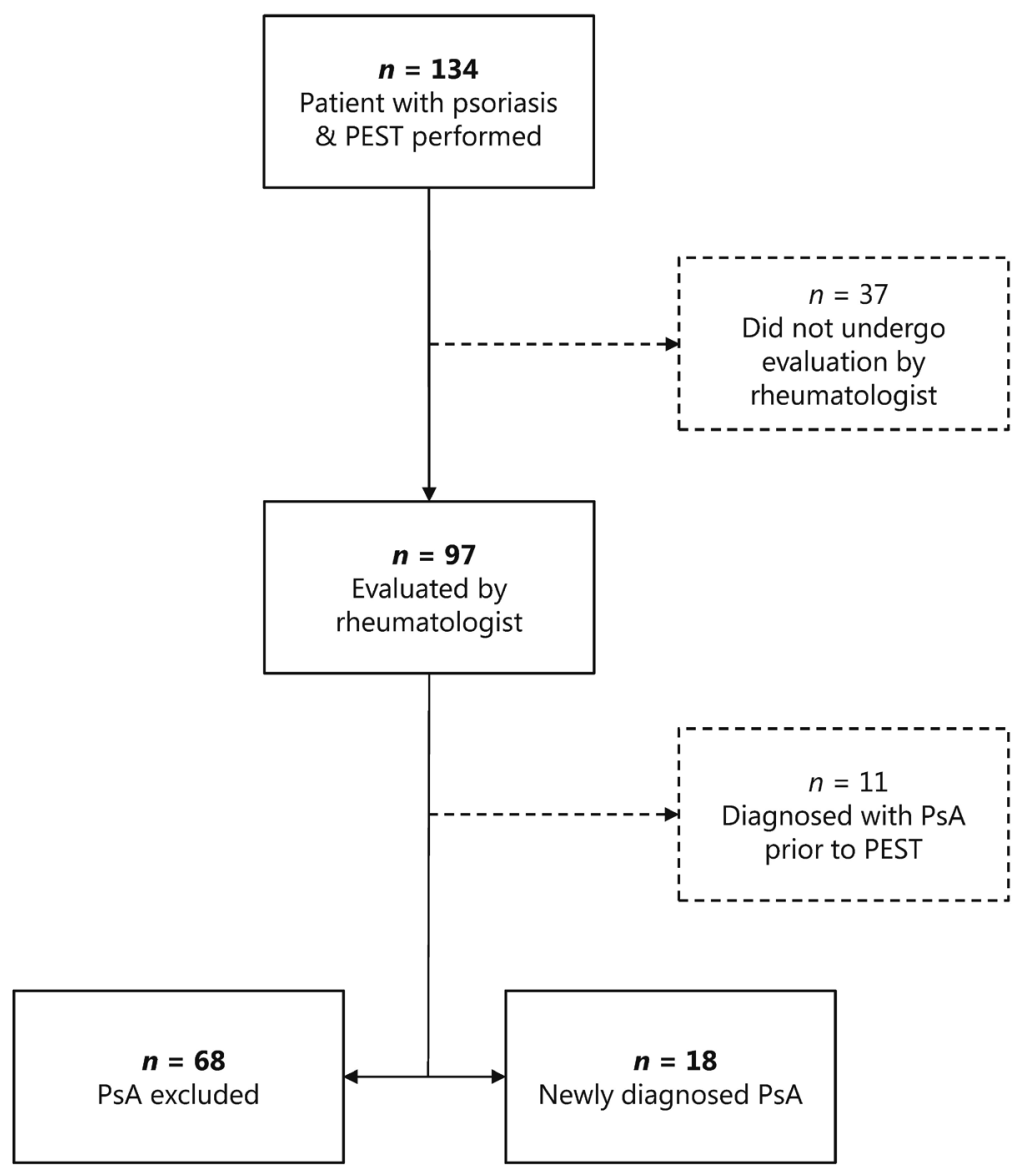

Figure 1. Flow-diagram showing the psoriasis patients that performed a Psoriasis Epidemiology Screening Tool (PEST) questionnaire in the study period reviewed. PSA, psoriatic arthritis. 
The PEST scores and diagnostic efficacy of the test are shown in Table 1. The PEST cut-off of $\geq 3$ points had an AUC of 0.70 [95\% confidence interval (CI) 0.60-0.80] and the PEST cut-off of $\geq 2$ points increased the AUC to 0.76 ( $95 \% \mathrm{CI} 0.66-0.85$ ), but this difference was not statistically significant. Compared to the original PEST cutoff $\geq 3$ points, the alternative PEST cut-off $\geq 2$ points markedly improved the test sensitivity. If the newly proposed lower cutoff score was to be used as a decisionmaking tool for referral, this would result in much fewer cases of PsA being missed, at the cost of more patients needing to be screened by a rheumatologist. The 11 patients with a previously established diagnosis of PSA in whom the PEST was performed had high PEST scores, whereas the 37 patients with psoriasis who opted out of clinical evaluation by a rheumatologist had low PEST scores (Table 1). It is reasonable to assume that those 37 patients had fewer musculoskeletal complaints and lower prevalence of PsA, indicating that our results may have overestimated the true prevalence of newly diagnosed PsA while at the same time underestimating the true test efficacy of the PEST.

Table 1.

\begin{tabular}{lcccc}
\hline PEST score & $\begin{array}{l}\text { Psoriasis: } \\
\text { PsA excluded }\end{array}$ & $\begin{array}{l}\text { PsA: } \\
\text { newly diagnosed }\end{array}$ & $\begin{array}{l}\text { PsA: } \\
\text { previously diagnosed }\end{array}$ & $\begin{array}{c}\text { Unknown: } \\
\text { not evaluated }\end{array}$ \\
\hline $\mathbf{0}$ & 26 & 1 & 0 & 16 \\
$\mathbf{1}$ & 17 & 1 & 2 & 15 \\
$\mathbf{2}$ & 15 & 6 & 1 & 4 \\
$\mathbf{3}$ & 9 & 6 & 1 & 2 \\
$\mathbf{4}$ & 0 & 4 & 5 & 0 \\
$\mathbf{5}$ & 1 & 0 & $\mathbf{1 1}$ & 0 \\
$\begin{array}{l}\text { Total } \\
\text { patients (n) }\end{array}$ & $\mathbf{6 8}$ & $\mathbf{1 8}$ & & $\mathbf{3 7}$ \\
& & & $\mathbf{P P V}$ & $\mathbf{N P V}$ \\
\hline $\begin{array}{l}\text { Cut-off using } \\
\text { score } \geq \mathbf{3}^{\mathrm{b}}\end{array}$ & $\mathbf{5 6 \% ( 1 0 / 1 8 )}$ & $\mathbf{8 5 \% ( 5 8 / 6 8 )}$ & $\mathbf{5 0 \% ( 1 0 / 2 0 )}$ & $\mathbf{8 8 \% ( 5 8 / 6 6 )}$ \\
$\begin{array}{l}\text { Cut-off using } \\
\text { score } \geq \mathbf{2}^{\mathrm{b}}\end{array}$ & $\mathbf{8 9 \% ( 1 6 / 1 8 )}$ & $\mathbf{6 3 \% ( 4 3 / 6 8 )}$ & $\mathbf{3 9 \% ( 1 6 / 4 1 )}$ & $\mathbf{9 6 \% ( 4 3 / 4 5 )}$ \\
\hline
\end{tabular}

Table 1. Evaluation of the Psoriasis Epidemiology Screening Tool (PEST) scores. The results of the PEST score are shown based on the groups shown in Figure 1. For instance, in the patients with psoriasis in whom PSA was excluded, 17 patients had a PEST score result of 1, and 15 had a PEST score results of 2. The sensitivity, specificity, PPV and NPV were calculated based only on the 18 patients with newly

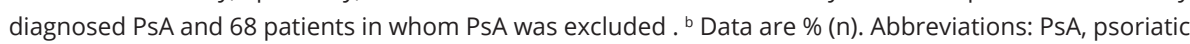
arthritis; PPV, positive predictive value; NPV, negative predictive value. 
Clinical features were not different between patients with newly diagnosed PSA compared with patients in whom PsA was excluded, but there was a trend for more frequent psoriatic nail involvement and longer psoriasis disease duration in patients with newly diagnosed PsA (Table S1; see Supporting Information). Of the patients with newly diagnosed PsA, 15 patients (83\%) had arthritis and three (17\%) already had erosive disease at presentation (Table S1; see Supporting Information).

Of the psoriasis patients with false-positive PEST scores (cut-off $\geq 2$ ), the most common finding was osteoarthritis in nine patients and an additional eight reported a history suspicious for past enthesitis (typically at the Achilles site) or arthritis in whom no inflammatory articular disease could be detected during clinical evaluation. Of the patients with PSA with false-negative PEST scores, one had enthesitis at the lateral epicondyle and the other monoarthritis of the wrist.

Overall, our results are very much in line with the recently published data by Coates et al.,(1) thereby providing additional validity of these observations. Using the PEST as the sole determinant to refer patients may place a greater burden on the capacity of the rheumatologist, but there are ways of overcoming this challenge. Firstly, the PEST should be considered a first step, following which the patient and dermatologist must decide if referral to the rheumatologist is clinically necessary. The future discovery of biomarkers capable of identifying patients with PsA, in particular those that require 'tight control', could enhance the specificity of referrals. Secondly, considering the high prevalence of $\mathrm{PsA}_{2}(9)$ as similarly found in our study, we may need to change the way of providing care to our patients with psoriasis. Rather than approaching them from a rheumatologists' joint or dermatologists' skin perspective, a patient-centred approach may overcome the hurdle of referring patients back and forth, and more effort should be made in finding the optimal method of doing so in clinical practice. $(10,11)$

In conclusion, using the PEST at lower cut-off score will provide the dermatologist with a simple, first screening tool to more confidently exclude PsA. 


\section{References:}

1. Coates LC, Savage L, Waxman R, Moverley AR, Worthington S, Helliwell PS. Comparison of screening questionnaires to identify psoriatic arthritis in a primary care population: a cross sectional study. Br J Dermatol. 2016;175:542-8.

2. Sokoll KB, Helliwell PS. Comparison of disability and quality of life in rheumatoid and psoriatic arthritis. J Rheumatol. 2001 Aug;28(8):1842-6.

3. Helliwell PS, Ruderman EM. Natural History, Prognosis, and Socioeconomic Aspects of Psoriatic Arthritis. Rheum Dis Clin North Am. 2015;41(4):581-91.

4. Mease PJ, Gladman DD, Helliwell P, Khraishi MM, Fuiman J, Bananis E, et al. Comparative performance of psoriatic arthritis screening tools in patients with psoriasis in European/ North American dermatology clinics. J Am Acad Dermatol. 2014;71(4):649-55.

5. Ibrahim GH, Buch MH, Lawson C, Waxman R, Helliwell PS. Evaluation of an existing screening tool for psoriatic arthritis in people with psoriasis and the development of a new instrument: The Psoriasis Epidemiology Screening Tool (PEST) questionnaire. Clin Exp Rheumatol. 2009;27(3):469-74.

6. Coates LC, Aslam T, Al Balushi F, Burden a D, Burden-Teh E, Burden-The E, et al. Comparison of three screening tools to detect psoriatic arthritis in patients with psoriasis (CONTEST study). Br J Dermatol. 2013 Apr;168(4):802-7.

7. Taylor W, Gladman D, Helliwell P, Marchesoni A, Mease P, Mielants H. Classification criteria for psoriatic arthritis: development of new criteria from a large international study. Arthritis Rheum. 2006 Aug;54(8):2665-73.

8. DeLong ER, DeLong DM, Clarke-Pearson DL. Comparing the areas under two or more correlated receiver operating characteristic curves: a nonparametetric approach. Biometrics. 1988;44(3):837-45.

9. Villani AP, Rouzaud M, Sevrain M, Barnetche T, Paul C, Richard M-A, et al. Prevalence of undiagnosed psoriatic arthritis among psoriasis patients: Systematic review and metaanalysis. J Am Acad Dermatol. 2015 Aug;73(2):242-8.

10. Cobo-Ibáñez T, Villaverde V, Seoane-Mato D, Muñoz-Fernández S, Guerra M, Del Campo PD, et al. Multidisciplinary dermatology-rheumatology management for patients with moderate-tosevere psoriasis and psoriatic arthritis: a systematic review. Rheumatol Int. 2015;36(2):221-9.

11. Warren RB, Helliwell PS, Chinoy H. Time for a "joint" approach? Br J Dermatol. 2013;168(4): 683-4. 


\begin{tabular}{lcc}
\hline & $\begin{array}{c}\text { Psoriasis, } \\
\text { PsA excluded }\end{array}$ & $\begin{array}{c}\text { PsA, } \\
\text { newly diagnosed }\end{array}$ \\
\hline Age (years) & $49 \pm 17$ & $48 \pm 11$ \\
Female & $47 \%$ & $33 \%$ \\
Psoriasis duration (years) & $16 \pm 13$ & $23 \pm 13$ \\
Psoriasis severity (PASI) & $4.6(0-19)$ & $2.2(0-19)$ \\
Psoriatic nail involvement & $58 \%$ & $81 \%$ \\
Non-vulgaris skin type & $22 \%$ & $18 \%$ \\
Cutaneous therapy only & $62 \%$ & $56 \%$ \\
UV-therapy & $13 \%$ & $17 \%$ \\
Systemic drug use & $12 \%$ & $22 \%$ \\
Biological drug use & $16 \%$ & $22 \%$ \\
CRP & $2.2(0.50-55)$ & $2.0(0.60-3.2)$ \\
ESR & $6.0(1.0-54)$ & $7.0(2.0-14)$ \\
Erosive at presentation & - & $17 \%$ \\
Arthritis at presentation & - & $83 \%$ \\
Joint count at presentation & - & $2(0-7)$ \\
Enthesitis only at presentation & - & $17 \%$ \\
Dactylitis at presentation & $-11 \%$ \\
Axial involvement & $-1 \% \%$ \\
\hline
\end{tabular}

Supplementary Table 1, Clinical and demographic features: Data are shown as mean \pm standard deviation, median (range), or frequency (\%). The therapies shown represent therapy at the time of the PEST questionnaire. Systemic drug use refers to non-biologicals, such as methotrexate and fumaric acid. There were no significant group differences (at $p<0.05$ ), but the newly diagnosed PsA group had a trend for longer disease duration (t-test, $p=0.07$ ) and more frequent psoriatic nail involvement (Chi square, $p=0.08)$. 


$$
3
$$




\section{CHAPTER 3}

\section{First-line csDMARD monotherapy drug retention in psoriatic arthritis: methotrexate outperforms sulfasalazine}

Authors:

Marleen E. Jacobs ${ }^{1,2}$, Juliëtte N. Pouw ${ }^{1,2}$, Paco Welsing1, Timothy R.D.J. Radstake TRDJ ${ }^{1,2}$, Emmerik F.A. Leijten ${ }^{1,2}$

Affiliations:

1. Department of Rheumatology and Clinical Immunology, UMC Utrecht, the Netherlands 2. Center for Translational Immunology, UMC Utrecht, the Netherlands

Published in:

Rheumatology (Oxford). 2020 Aug. Online ahead of print doi: 10.1093/rheumatology/keaa399 


\section{Abstract}

Background: Conventional synthetic disease modifying anti-rheumatic drugs (csDMARDs) are the first-line treatment for psoriatic arthritis (PsA), but there is conflicting data regarding their efficacy and scarce reports describing the duration of use (drug retention) of CSDMARD in this population. Their position in treatment recommendations is a matter of growing debate due to the availability of alternative treatment options with higher levels of evidence. We aimed to study drug retention and predictors for drug retention among PSA patients receiving first-line csDMARD monotherapy.

Methods: Retrospective cohort study in DMARD-naïve adult PsA patients in whom a first CSDMARD was prescribed as monotherapy primarily to treat PsA-related symptoms. The main outcome was time to failure of the cSDMARD (i.e stopping the cSDMARD or adding another DMARD).

Results: A total of 187 patients were included, who were mainly prescribed methotrexate (MTX) $(n=163)$ or sulfasalazine (SSZ) $(n=21)$. The pooled median drug retention time was 31.8 months (interquartile range 9.04-110). Drug retention was significantly higher in MTX (median 34.5 months; interquartile range 9.60123) as compared to SSZ-treated patients (median 12.0 months; interquartile range 4.80-55.7)( $p=0.016$, log-rank test). In multivariable Cox regression, the use of MTX and older age were associated with increased retention. The main reasons for treatment failure were inefficacy (52\%) and side-effects (28\%). Upon failure, MTX treated patients were more commonly, subsequently treated with a biologic DMARD compared to SSZ $(p<0.05)$.

Conclusion: MTX outperforms SSZ as first-line cSDMARD in DMARD-naïve PSA patients with respect to monotherapy drug retention in daily clinical practice. 


\section{Introduction}

Psoriatic arthritis (PSA) is a chronic, inflammatory musculoskeletal disorder, which develops in approximately one in ten patients with psoriasis and often leads to a decreased quality of life and impaired function (1). Currently, conventional synthetic disease modifying anti-rheumatic drugs (csDMARDs) are the most commonly prescribed drugs as first-line treatment for peripheral arthritis in PSA, as recommended by the international EULAR and GRAPPA guidelines $(2,3)$. These guidelines refer to methotrexate (MTX), sulfasalazine (SSZ), leflunomide (LEF) and ciclosporin A as possible treatment options. However, previous studies found little or no effect of MTX on psoriatic synovitis, and higher effectiveness of tumour necrosis factor (TNF) inhibitors compared to MTX in reducing radiographic progression in PSA patients (4-9). Efficacy of LEF in PSA has been shown in one randomized controlled trial (10). Regarding SSZ, clinical trials have found a modest favourable effect on musculoskeletal symptoms (11-13).

While there is a lack of high level evidence to support the use of cSDMARD efficacy in PSA, CSDMARD drug retention rates can provide indirect evidence. One large study found a two-year retention rate of MTX therapy of $\sim 65 \%$ in both rheumatoid arthritis (RA) and PSA, suggestive of a beneficial effect of MTX in PSA (14). Another comparable study observed mean MTX and LEF drug retention of 13 and 6 years, respectively (15). More indirect evidence comes from the fact that PsA has historically been treated similar to RA, where high-level evidence supports the use of cSDMARDs, and justifies this treatment in PSA patients. Other arguments to consider cSDMARD therapy include well described long-term safety outcomes and low costs; a factor taken into account by some (inter)national guidelines.

Despite these reasons to treat PSA with CSDMARDs, their position is under pressure due to alternative treatment options with higher levels of evidence (1). In line, a recent guideline recommends the use of TNF-inhibitors as a first-line treatment (16). Furthermore, in daily practice csDMARD side effects are commonly reported which negatively impact drug retention.

This study aimed to evaluate the level of indirect evidence for cSDMARD efficacy by describing first-line cSDMARD monotherapy drug retention for treating PSA in daily clinical practice, comparing the retention rate of different csDMARDs and investigating possible predictors of drug retention. 


\section{Methods}

\section{Study design}

This retrospective cohort study was performed at the University Medical Centre Utrecht, the Netherlands, and approved by the local institutional review board. The first selection of eligible patients was performed via electronic search based on diagnosis and diagnosis related groups (DRGs). Manual screening was performed twice to ensure eligibility. Inclusion criteria were (1) clinical diagnosis of PsA and (2) DMARD-naïve (no prior DMARD therapy for any cause, including psoriasis), and (3) initiated DMARD as monotherapy after January $1^{\text {st }} 2000$. Patients were excluded if CSDMARD therapy was primarily initiated for treating extra-articular manifestations (e.g. to treat psoriasis). All patient data was encrypted and saved using the online database CastorEDC.

\section{Outcome measures}

The main outcome was defined as the first-line csDMARD monotherapy drug retention time. The first-line cSDMARD monotherapy cessation date (abbreviated "CSDMARD monotherapy failure") was set at the last recorded date during which the first-line cSDMARD was prescribed as monotherapy. Thus, discontinuation of cSDMARD monotherapy occurred upon (i) cessation of the first-line cSDMARD therapy or (ii) continuing the first-line csDMARD but adding a bDMARD or second CSDMARD. Observations were considered "censored data" if the patient was still on therapy at the last known medical record observation point or if the patient was lost to follow-up.

Using pre-defined categories the research team retrospectively identified the main reason for first-line treatment cessation, as based on the reason recorded by the treating physician in the medical record. Categories included remission, inefficacy, side-effects, (planned) pregnancy and other reasons. We registered the subsequent treatment prescribed within a window of six months after cSDMARD monotherapy failure. A maximum tolerated "drug holiday" of three months was allowed to mimic clinical care. Demographic, clinical and radiographic parameters were collected to identify predictors of treatment response.

\section{Statistical analysis}

Data analyses were performed using SPSS software (version 25.0). Data were represented as mean and standard deviation (SD) for normally distributed data and median and interquartile range (IQR) for non-normally distributed data. Baseline characteristics between MTX and SSZ groups were compared using 
the independent samples T-test (normally distributed data), Mann-Whitney $U$ test (non-normally distributed data) or Chi-square test as appropriate. A P-value of less than 0.05 was considered statistically significant. Drug retention was described using Kaplan-Meier plots and statistically compared using the log-rank test. Potential predictors of drug retention were studied using a multivariable cox model (described in Supplementary Methods).

\section{Results}

\section{Cohort characteristics}

In total, 187 patients with PsA met the inclusion criteria. Main demographics and disease activity characteristics are shown in Supplementary Table S1. The cohort consisted of $68 \%$ males with mean age 48 years (SD 13.3). The duration of disease was 0.4 years (IQR 0.1-1.0) and 7.5 years (IQR 2.1-18.1) for PsA and psoriasis, respectively. The most commonly prescribed first-line csDMARD was MTX (87\%), followed by SSZ (11\%) and LEF (2\%). As compared to SSZ, patients initiating MTX had significantly higher age, body mass index and swollen and tender joint count. Also, there was a trend for erosive disease to be more common in the MTX than SSZ group.

\section{CSDMARD monotherapy drug retention}

In total, 132 patients (71\%) failed their first line therapy during follow-up, while 55 patients $(29 \%)$ had censored observation. The monotherapy drug retention showed a large drop in retention early after treatment initiation. In the entire study population, the median monotherapy drug retention was 31.8 months (95\% Cl 18.9-44.6; IQR 9.04-110). At 12 months after treatment initiation, 70\% of patients were still using the first-line csDMARD as monotherapy.

We next compared the different cSDMARDs initiated, excluding LEF from further analysis due to low numbers. MTX had significantly higher monotherapy drug retention as compared to SSZ ( $p=0.016)$ (Figure 1; Supplementary Figure S1). For MTX the median monotherapy drug retention was 34.5 months ( $95 \% \mathrm{Cl} 22.2-46.8$; IQR 9.60-123). For SSZ the median monotherapy drug retention was 12.0 months (95\% Cl 4.32-19.8; IQR 4.80-55.7). At 12 months, 72\% of patients that initiated MTX were still using MTX as monotherapy, whereas $52 \%$ of patients that initiated SSZ were still using SSZ as monotherapy. 
Based on univariable cox regression analysis the DMARD initiated was significantly associated with DMARD retention, where MTX-initiated patients had better retention as compared to SSZ-initiated patients (HR 0.545 (95\% Cl 0.330-0.899), $\mathrm{p}=0.017)$. In addition, older age increased csDMARD monotherapy retention (HR 0.985 per year age increase $(95 \% \mathrm{Cl} 0.971-0.998), p=0.026)$. When incorporating age and csDMARD initiated into multivariable cox regression model there was a non-significant trend for longer drug retention in MTX group [HR $0.630((\mathrm{Cl} 0.372-$ 1.069), $p=0.087$ ] and older patients [per year age increase HR $0.988(\mathrm{Cl} 0.974$ 1.002), $p=0.095]$. We next screened for potential predictors of drug retention in a multivariable cox model: the final model included csDMARD-initiated and age as the only predictors of drug retention (same HR as above).

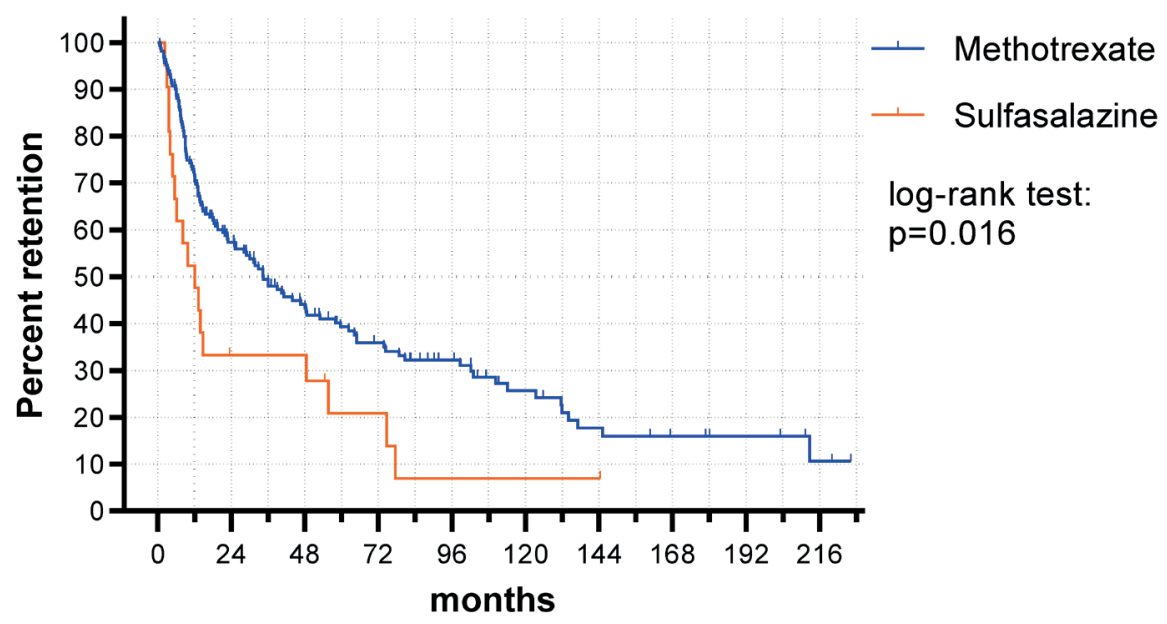

Figure 1. csDMARD monotherapy drug retention in PsA.

Kaplan-Meier plot shows monotherapy drug retention rate of MTX or SSZ prescribed as first-line treatment in DMARD-naïve PsA patients. Ticks indicate censored data. MTX showed significantly higher monotherapy drug retention as compared to SSZ. cSDMARD: conventional synthetic DMARD.

\section{Cause of CSDMARD monotherapy failure}

The main reason for csDMARD monotherapy treatment cessation was treatment inefficacy (52\%), followed by side-effects (28\%) (Figure 2). The main reasons for treatment cessation between MTX and SSZ were slightly different, with more patients that stopped MTX due to (planned) pregnancy and more patients that stopped SSZ due to inefficacy (Supplementary Table S2). Remission occurred in 11 patients that initiated MTX and 2 patients that initiated SSZ. Retention analysis remained similar when remission cases were excluded or considered censored. 
The most important side effects were gastrointestinal complaints (32\%) and general malaise (24\%) (Figure 2). Patients treated with MTX reported more side effects than patients treated with SSZ (Supplementary Table S3 and S4). At time of cSDMARD monotherapy failure, the patients that failed due to inefficacy had more active disease than the patients that failed due to other reasons (Supplementary Table S5).

\section{Main reason for csDMARD monotherapy retention failure}

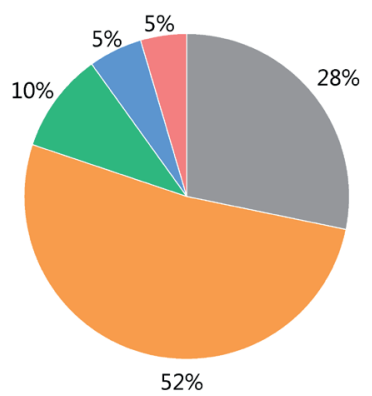

\section{Main side effect leading to csDMARD cessation}

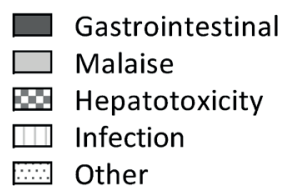

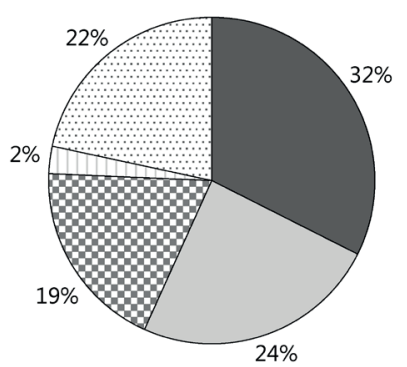

Figure 2. reason for csDMARD monotherapy retention failure Top: Main reasons for csDMARD monotherapy retention failure (non-censored cases, n=132). Bottom: Main side effect reported at stop date for patients in whom the primary reason for cSDMARD cessation was side effects (non-censored data, $n=37$ ). cSDMARD: conventional synthetic DMARD. 


\section{Follow-up treatment upon CSDMARD monotherapy failure}

Upon csDMARD monotherapy failure, the first-line cSDMARD was most commonly switched to a different csDMARD (27\%) or a bDMARD was added (25\%). However, the follow-up treatment regimen was significantly different between the MTX and SSZ groups: MTX treated patients were more commonly prescribed a bDMARD upon failure $(p<0.05)$. In addition, failure due to side effects versus inefficacy resulted in different follow-up treatment strategies: a bDMARD was prescribed in $55 \%$ of patients that failed due to inefficacy as compared to $11 \%$ of patients that failed due to side effects $(p<0.05)$ (Supplementary Figure $\mathbf{2}$ and Supplementary Table S6-S8).

\section{Discussion}

This study shows that MTX as a first-line csDMARD for treating peripheral arthritis in PsA has higher monotherapy drug survival than SSZ. For all csDMARDs, monotherapy drug retention shows a large drop in the first year of treatment. Inefficacy is most commonly seen as reason for drug cessation, followed by side-effects. The results from this study are derived from a real-world setting and display a realistic clinical scenario of the first-line cSDMARD monotherapy retention in cSDMARD-naïve PSA patients.

A limited number of previous studies have evaluated cSDMARD monotherapy drug retention $(14,15,17)$. We found a median csDMARD monotherapy retention of approximately 2.5 years, but witnessed a large drop in drug retention within the first year of treatment. One previous study found a 10-year MTX retention rate of more than $50 \%$, which largely exceeds our 10-year retention rate of around $25 \%$. This difference may be partly explained by the concomitant steroid use (15). Overall, the validity of our results are strengthened by those of other studies that found a similar drug retention rate $(14,17)$. Our data also reveal that-even in the presence of potential efficacy-side effects were reported in more than $50 \%$ of patients at the moment of CSDMARD monotherapy failure. Although not all of these side effects were deemed the principle cause of failure, they may have contributed to the modification of the treatment regimen.

With regards to predictors of csDMARD survival, one study described a larger PsA study cohort treated with MTX, but a shorter follow-up period with a maximum of 2 years. Their regression analysis showed age, disease duration, and patient reported outcomes to be significant predictors of MTX drug retention (14). We also found that older age was a predictor of longer drug retention, but did not identify other clinical parameters to be associated with drug retention. Additionally, we 
didn't find sex or C-reactive protein (CRP) levels to be significant predictors of drug retention, as proposed in earlier cohort studies $(15,18)$.

This study has a number of limitations. An important limitation is the retrospective nature of the study. Another factor that needs to be taken into account is the small number of subjects in the SSZ group compared to the MTX group. Also, drug retention is an assumed indirect measure of treatment efficacy, while drug adherence and treatment modifications are dependent on multiple factors in daily practice. Nonetheless, the use of real-world data also contains advantages over trial data by better portraying the setting in which DMARDs are initiated, as exemplified by the relative low joint count in our study cohort as compared to patients enlisted in PsA trials.

Overall our results support the use of MTX as first-line therapy in treating peripheral arthritis in PSA, as recommended by current EULAR and GRAPPA guidelines(2,3). These data show that, at least compared to SSZ, MTX performs better with respect to monotherapy drug retention. Considering the emergence of numerous novel drugs for treating psoriatic arthritis, prospective studies (e.g. pragmatic randomized clinical trials (19)) are required to further elucidate the differential efficacy of specific csDMARDs as first line treatment in PsA. The future research agenda should continue to focus on treatment challenges faced in the real-world setting, where the largest group of patients with PsA present with early, mono- or oligoarticular disease.

In conclusion, we found that MTX outperforms SSZ as first-line cSDMARD in DMARD-naïve PSA patients with respect to monotherapy drug retention in daily clinical practice. Future prospective studies should further elucidate the efficacy of csDMARDs as first-line treatment for PsA. 


\section{References}

1. Ritchlin CT, Colbert RA, Gladman DD. Psoriatic Arthritis. N Engl J Med. 2017 Mar 9;376(10): 957-70.

2. Gossec L, Smolen JS, Ramiro S, de Wit M, Cutolo M, Dougados M, et al. European League Against Rheumatism (EULAR) recommendations for the management of psoriatic arthritis with pharmacological therapies: 2015 update. Ann Rheum Dis. 2016 Mar;75(3):499-510.

3. Coates LC, Kavanaugh A, Mease PJ, Soriano ER, Laura Acosta-Felquer M, Armstrong AW, et al. Group for Research and Assessment of Psoriasis and Psoriatic Arthritis 2015 Treatment Recommendations for Psoriatic Arthritis. Arthritis Rheumatol. 2016; 68:1060-71

4. Mahmood F, Coates LC, Helliwell PS. Current concepts and unmet needs in psoriatic arthritis. Clin Rheumatol. 2018;37(2):297-305.

5. Eder L, Thavaneswaran A, Chandran V, Gladman DD. Tumour necrosis factor a blockers are more effective than methotrexate in the inhibition of radiographic joint damage progression among patients with psoriatic arthritis. Ann Rheum Dis. 2014 Jun 1;73(6):1007-11.

6. Marchesoni A, Lubrano E, Cauli A, Ricci M, Manara M. Psoriatic Disease: Update on Traditional Disease-modifying Antirheumatic Drugs. J Rheumatol. 2015 Nov;93:61 LP - 64.

7. Kingsley GH, Kowalczyk A, Taylor H, Ibrahim F, Packham JC, McHugh NJ, et al. A randomized placebo-controlled trial of methotrexate in psoriatic arthritis. Rheumatology. 2012 Feb;51(8):1368-77.

8. Mease PJ, Gladman DD, Collier DH, Ritchlin CT, Helliwell PS, Liu L, et al. Etanercept and Methotrexate as Monotherapy or in Combination for Psoriatic Arthritis: Primary Results From a Randomized, Controlled Phase III Trial. Arthritis Rheumatol (Hoboken, NJ). 2019 Jul;71(7):1112-24.

9. Wilsdon TD, Whittle SL, Thynne TR, Mangoni AA. Methotrexate for psoriatic arthritis. Cochrane Database Syst Rev. 2019 Jan;(1).

10. Kaltwasser JP, Nash P, Gladman D, Rosen CF, Behrens F, Jones P, et al. Efficacy and safety of leflunomide in the treatment of psoriatic arthritis and psoriasis: A multinational, doubleblind, randomized, placebo-controlled clinical trial. Arthritis Rheum. 2004 Jun;50(6):1939-50.

11. Clegg DO, Reda DJ, Mejias E, Cannon GW, Weisman MH, Taylor T, et al. Comparison of sulfasalazine and placebo in the treatment of psoriatic arthritis. A Department of Veterans Affairs Cooperative Study. Arthritis Rheum. 1996 Dec;39(12):2013-20.

12. Gupta AK, Grober JS, Hamilton TA, Ellis CN, Siegel MT, Voorhees JJ, et al. Sulfasalazine therapy for psoriatic arthritis: a double blind, placebo controlled trial. J Rheumatol. 1995 May;22(5):894-8.

13. Dougados $M$, vam der Linden $S$, Leirisalo-Repo $M$, Huitfeldt $B$, Juhlin $R$, Veys $E$, et al. Sulfasalazine in the treatment of spondylarthropathy. A randomized, multicenter, doubleblind, placebo-controlled study. Arthritis Rheum. 1995 May;38(5):618-27.

14. Lie E, van der Heijde D, Uhlig T, Heiberg MS, Koldingsnes W, Rødevand E, et al. Effectiveness and retention rates of methotrexate in psoriatic arthritis in comparison with methotrexatetreated patients with rheumatoid arthritis. Ann Rheum Dis. 2010 Apr;69(4):671-6. 
15. Zaffarana CA, Cerda O, Schneeberger EE, Carrillo I, Gonzalez MC, Citera G. Methotrexate and leflunomide survival in patients with psoriatic arthritis. Int J Clin Rheumtol. 2018;13(2):102.

16. Singh JA, Guyatt G, Ogdie A, Gladman DD, Deal C, Deodhar A, et al. 2018 American College of Rheumatology/National Psoriasis Foundation Guideline for the Treatment of Psoriatic Arthritis. Arthritis Care Res (Hoboken). 2018 Nov;

17. Ricci M, De Marco G, Desiati F, Mazzocchi D, Rotunno L, Battafarano N, et al. [Long-term survival of methotrexate in psoriatic arthritis]. Reumatismo. 2009;61(2):125-31.

18. Glintborg B, Østergaard M, Dreyer L, Krogh NS, Tarp U, Hansen MS, et al. Treatment response, drug survival, and predictors thereof in 764 patients with psoriatic arthritis treated with anti-tumor necrosis factor a therapy: Results from the nationwide Danish DANBIO registry. Arthritis Rheum. 2011 Feb;63(2):382-90.

19. Gamerman V, Cai T, Elsäßer A. Pragmatic randomized clinical trials: best practices and statistical guidance. Heal Serv Outcomes Res Methodol. 2019;19(1):23-35. 


\section{Supporting information}

\section{Supplementary methods}

\section{Multivariable cox regression in a multivariable cox model}

As this was not a randomized study, correction for possible confounders influencing the difference in retention between MTX and SSZ was necessary. Therefore, any clinical factor considered potentially different between MTX and SSZ groups and related to drug retention (variables: sex, age, smoker, disease duration, joint count, dactylitis, psoriasis phenotype, erythrocyte sedimentation rate, erosive disease) were evaluated in the multivariable Cox regression. To optimally correct for confounding while keeping a parsimonious model, potential confounders were added to the model one by one. At each step, the necessity to control for the variable was determined. A change in the regression coefficient for treatment effect of more than $10 \%$ led to retention of the variable in the model.

In a separate analysis, we screened for potential predictors of drug retention using a multivariable cox model, by entering blocks of variables, starting with DMARD group, followed by demographic factors, followed by clinical factors. Per block the least significant variables were removed while retaining variables with a nominal $p$ value of $<0.15$. The influence of variables in the final cox models are described as hazard ratio (HR) with 95\% confidence interval $(\mathrm{Cl})$ and $\mathrm{p}$ value.

\section{csDMARD monotherapy drug retention}

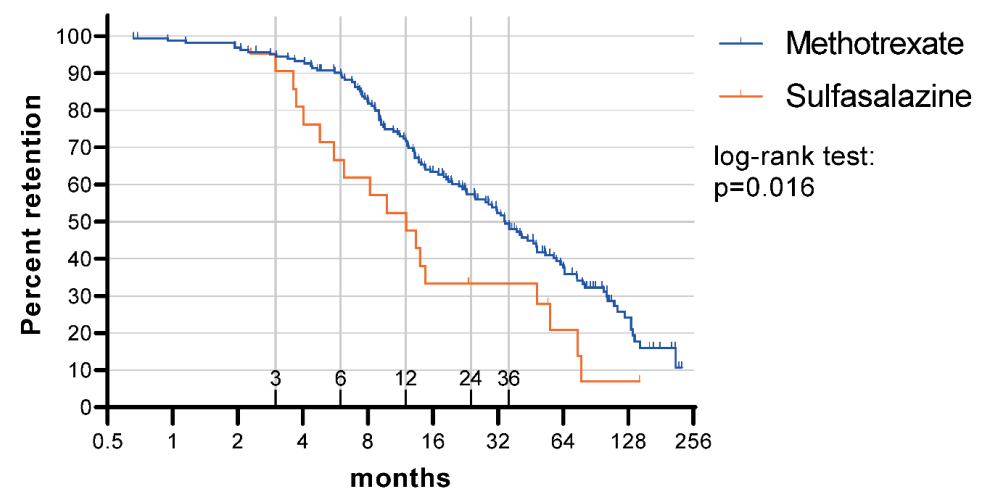

Supplementary Figure S1. csDMARD monotherapy drug retention in PSA.

Same data is shown as in Figure 1, but the graph is displayed with the X-axis as logarithmic scale to visualize the early retention rate. Kaplan-Meier plot is shown for methotrexate (MTX) or sulfasalazine (SSZ) as first-line cSDMARD to treat psoriatic arthritis. Ticks indicate censored data. MTX had significantly higher monotherapy drug survival as compared to SSZ ( $p=0.016$, log-rank test). 


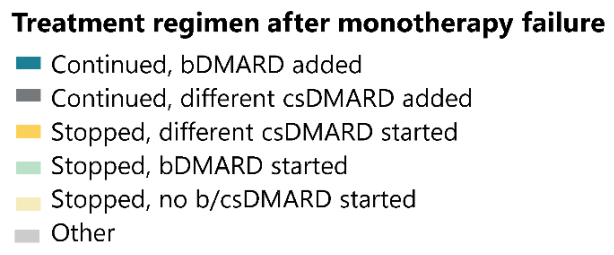

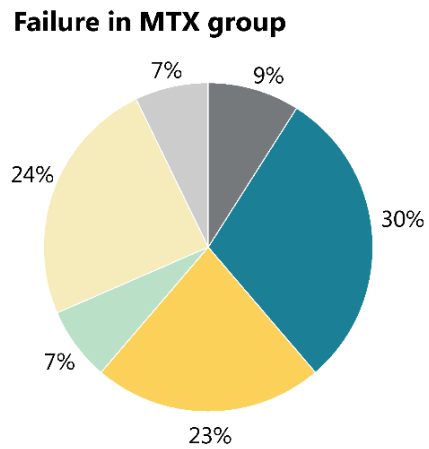

Failure due to inefficacy

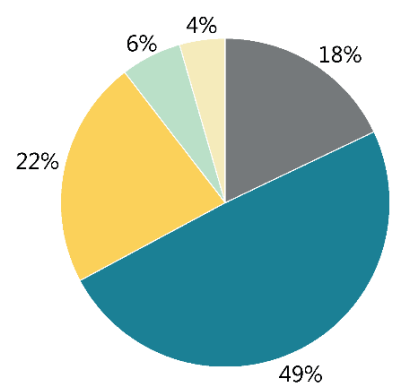

Failure in SSZ group

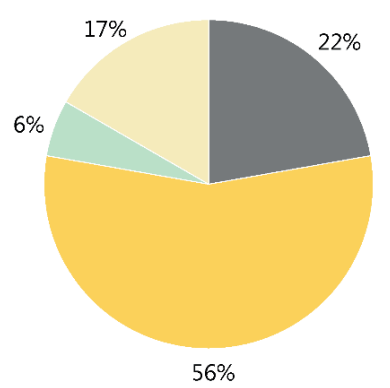

Failure due to side effects

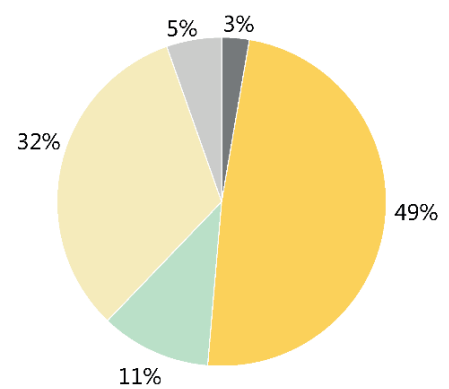

\section{Supplementary Figure S2. Follow-up treatment}

Follow-up treatment regimen the first six months after the monotherapy cSDMARD treatment failure date, in all non-censored cases.

Top Left: In patients that initiated MTX $(n=111)$.

Top Right: In patients that initiated SSZ ( $\mathrm{n}=18)$.

Bottom left: In patients with inefficacy as main treatment failure cause (regardless of treatment) $(n=68)$.

Bottom right: In patients with side effects as main treatment failure cause (regardless of treatment $(n=37)$ 


\begin{tabular}{|c|c|c|c|}
\hline & Total, $\mathbf{N}=187$ & MTX, N = 163 & $S S Z, N=21$ \\
\hline Male, N (\%) & $128(68)$ & $115(71)$ & $12(57)$ \\
\hline Age (years), mean $\pm S D^{*}$ & $48.3 \pm 13.3$ & $49.1 \pm 12.7$ & $39.5 \pm 13.4$ \\
\hline Body mass index, mean $\pm S D *$ & $26.7 \pm 4.5$ & $27.4 \pm 4.4$ & $25.3 \pm 3.5$ \\
\hline Smoker, N (\%) & $34 / 150(18.2)$ & $31 / 129(19.0)$ & $3 / 18(14.3)$ \\
\hline \multicolumn{4}{|l|}{ Disease duration (years), median (IQR) } \\
\hline PsA & $0.4(0.1-3.0)$ & $0.4(0.1-2.9)$ & $0.3(0.1-2.0)$ \\
\hline Psoriasis & $7.5(2.1-18.4)$ & $7.5(2.0-15.7)$ & $\begin{array}{l}10.8(3.1- \\
26.7)\end{array}$ \\
\hline \multicolumn{4}{|l|}{ Psoriasis phenotype, $\mathrm{N}(\%)^{\star}$} \\
\hline Vulgaris only & $130 / 185(69.5)$ & $117 / 161(71.8)$ & $10 / 21(47.6)$ \\
\hline Sine psoriasis & $31 / 185(16.6)$ & 21/161 (12.9) & $10 / 21(47.6)$ \\
\hline Other types ${ }^{1}$ & $24 / 185(12.8)$ & 23/161 (14.1) & $1 / 21(4.8)$ \\
\hline Nail disease present, N (\%) & $81 / 121(43.3)$ & $72 / 104(44.2)$ & 9/17 (42.9) \\
\hline Swollen joint count, median (IQR)* & $4(2-6)$ & $4(2-6)$ & $2(1-3)$ \\
\hline Tender joint count, median (IQR)* & $4(1-6)$ & $4(2-6)$ & $1(0-3)$ \\
\hline Dactylitis present, N (\%) & 18/161 (9.6) & $17 / 142(10.4)$ & $1 / 17(4.8)$ \\
\hline Axial disease present ${ }^{2}, \mathrm{~N}(\%)$ & $27 / 185$ (14.4) & 24/161 (14.7) & $3 / 21(14.3)$ \\
\hline CRP, median (IQR) & $8.0(2.9-16.0)$ & $8.0(3.0-15.0)$ & $\begin{array}{l}10.0(1.0- \\
26.0)\end{array}$ \\
\hline ESR, median (IQR) & $14.0(6.0-27.5)$ & $14.0(7.0-27.0)$ & $\begin{array}{l}16.0(6.0 \text { - } \\
31.0)\end{array}$ \\
\hline Erosive disease, N (\%) & $40 / 156(21.4)$ & $37 / 140(22.7)$ & $2 / 15(9.5)$ \\
\hline
\end{tabular}

Main demographic and clinical characteristics of the study cohort at time of csDMARD therapy initiation. The total cohort $(n=187)$ included 3 patients treated with leflunomide (data not shown separately). Psoriasis area and severity scores were unavailable for most cases and not shown. Descriptive data show the mean \pm SD, median (IQR) or $\mathrm{N}(\%)$.

${ }^{1}$ Other psoriasis types included guttate, palmoplantaris, inverse, and mixed types.

2 Presence/absence of axial disease was based on the clinical diagnosis from the treating physician.

* Clinical parameter is significantly different $(P<0.05)$ between MTX and SSZ

MTX: methotrexate; SSZ: sulfasalazine; CRP: C-reactive protein; ESR: erythrocyte sedimentation rate; PSA: psoriatic arthritis. 
Supplementary Table S2. Main reasons for csDMARD monotherapy retention failure for all non-censored cases.

\begin{tabular}{llll}
\hline & All. $\mathbf{N}=\mathbf{1 3 2}$ & MTX. $\mathbf{N}=\mathbf{1 1 1}$ & SSZ. $\mathbf{~}=\mathbf{1 8}$ \\
\hline Inefficacy, N (\%) & $68(51.5)$ & $55(49.5)$ & $12(66.7)$ \\
Side effects, N (\%) & $37(28.0)$ & $31(27.9)$ & $4(22.2)$ \\
Remission, N (\%) & $13(9.8)$ & $11(9.9)$ & $2(11.1)$ \\
(Planned) Pregnancy, N (\%) & $7(5.3)$ & $7(6.3)$ & $0(0.0)$ \\
Other reason, N (\%) & $7(5.3)$ & $7(6.3)$ & $0(0.0)$ \\
\hline
\end{tabular}

The total cohort $(n=132)$ included cases from 3 patients treated with leflunomide (data not shown separately).

Supplementary Table S3. Major side effect leading to csDMARD monotherapy retention failure.

\begin{tabular}{llll}
\hline & All. $\mathbf{N}=\mathbf{3 7}$ & MTX. N = 31 & SSZ. $\mathbf{N}=\mathbf{4}$ \\
\hline Gastrointestinal, N (\%) & $12(32.4)$ & $11(35.5)$ & $1(25.0)$ \\
Hepatotoxicity, N (\%) & $7(18.9)$ & $7(22.6)$ & $0(0.0)$ \\
Malaise, N (\%) & $9(24.3)$ & $8(25.8)$ & $1(25.0)$ \\
Infection, N (\%) & $1(1.7)$ & $0(0.0)$ & $0(0.0)$ \\
Other or unspecified side effect, N (\%) & $8(21.6)$ & $5(14.1)$ & $2(50.0)$ \\
\hline
\end{tabular}

Major side effect reported at stop date for patients in whom the primary reason for cessation was sideeffects (non-censored cases). The total cohort $(n=37)$ included 2 patients treated with leflunomide (data not shown separately).).

Supplementary Table S4. Major side effect reported at stop date for all non-censored cases

\begin{tabular}{llll}
\hline & All. $\mathbf{N}=\mathbf{1 3 2}$ & $\mathbf{M T X} . \mathbf{N}=\mathbf{1 1 1}$ & $\mathbf{S S Z} \mathbf{~ N ~ = ~ 1 8}$ \\
\hline No side effect, N (\%) & $56(42.4)$ & $45(40.5)$ & $10(55.6)$ \\
Gastrointestinal, N (\%) & $26(19.7)$ & $24(21.6)$ & $2(11.1)$ \\
Hepatotoxicity, N (\%) & $15(11.4)$ & $15(13.5)$ & $0(0.0)$ \\
Malaise, N (\%) & $13(9.8)$ & $11(9.9)$ & $2(11.1)$ \\
Infection, N (\%) & $4(3.0)$ & $3(2.7)$ & $0(0.0)$ \\
Other or unspecified side effect, N (\%) & $11(8.4)$ & $9(8.1)$ & $2(11.1)$ \\
Missing data, N (\%) & $6(4.5)$ & $4(3.6)$ & $2(11.1)$ \\
\hline
\end{tabular}

The total cohort $(n=132$ ) included cases from 3 patients treated with leflunomide (data not shown separately). The major side effects are shown for both patients in whom the primary reason for cessation was side-effects as well as for patients in whom the primary reason for cessation was another reason (e.g. treatment failure due to inefficacy, but where side-effects were also reported at the treatment failure date). 
Supplementary Table S5. Indicators of disease activity at time of csDMARD monotherapy failure date in all non-censored cases.

\begin{tabular}{|c|c|c|}
\hline & \multicolumn{2}{|c|}{$\begin{array}{l}\text { Reason for csDMARD } \\
\text { monotherapy retention failure }\end{array}$} \\
\hline & Inefficacy. $\mathrm{N}=68$ & Other. $N=64$ \\
\hline Swollen joint count, median (IQR)* & $3.00(1.00-5.00)$ & $0.00(0.00-1.50)$ \\
\hline Tender joint count, median (IQR)* & $4.00(0.75-8.00)$ & $0.00(0.00-2.00)$ \\
\hline CRP, median (IQR) & $8.00(3.00-14.00)$ & $3.00(0.80-15.50)$ \\
\hline ESR, median (IQR)* & $8.00(5.00-15.75)$ & $5.00(2.00-10.25)$ \\
\hline
\end{tabular}

Descriptive data show the median (IQR). Data of swollen joint count, tender joint count, CRP and ESR were available for 43 (63\%), 42 (62\%), 27 (40\%), 56 (82\%) patients in the inefficacy group, and for 33 (52\%), 35 (55\%), 13 (20\%) and 42 (66\%) patients in the 'other' group, respectively.

* Clinical parameter is significantly different $(P<0.05)$ between patients that stopped csDMARD treatment because of inefficacy compared to patients that stopped because of other reasons.

Supplementary Table S6. Follow-up treatment regimen the first six months after the monotherapy csDMARD treatment failure date.

\begin{tabular}{llll}
\hline & All. N = 132 & MTX. N = 111 & SSZ. N = 18 \\
\hline Continued, different csDMARD added, N (\%) & $14(10.6)$ & $10(9.0)$ & $4(22.2)$ \\
Continued, bDMARD added, N (\%) & $33(25.0)$ & $33(29.7)$ & $0(0.0)$ \\
Stopped, different csDMARD started, N (\%) & $36(27.3)$ & $25(22.5)$ & $10(55.6)$ \\
Stopped, bDMARD started, N (\%) & $9(6.8)$ & $8(7.2)$ & $1(5.6)$ \\
Stopped, no b/csDMARD started, N (\%) & $32(24.2)$ & $27(24.3)$ & $3(16.7)$ \\
Stopped, no b/csDMARD started, with less than & $4(3.0)$ & $4(3.6)$ & $0(0.0)$ \\
6 months follow up data, N (\%) & & & $0(0.0)$ \\
Other, N (\%) & $2(1.5)$ & $2(1.8)$ & $0(0.0)$ \\
Missing data, N (\%) & $2(1.5)$ & $2(1.8)$ & \\
\hline
\end{tabular}

The total cohort $(n=132$ ) included 3 patients treated with leflunomide (data not shown separately). The rows "continued" refer to the patients in whom the first-line csDMARD monotherapy was continued and an additional DMARD was prescribed as combination therapy. The rows "stopped" refer to the patients in which the first-line cSDMARD was stopped entirely. The follow-up treatment or lack thereof within a window of six months after monotherapy treatment failure date was recorded for non-censored cases. 
Supplementary Table S7. Follow-up treatment regimen upon inefficacy

\begin{tabular}{llll}
\hline & All. N = 68 & MTX. N = 55 & SSZ. N = 12 \\
\hline Continued, different csDMARD added, N (\%) & $12(17.6)$ & $8(14.5)$ & $4(33.3)$ \\
Continued, bDMARD added, N (\%) & $33(48.5)$ & $33(60.0)$ & $0(0.0)$ \\
Stopped, different csDMARD started, N (\%) & $15(22.1)$ & $6(10.9)$ & $8(66.7)$ \\
Stopped, bDMARD started, N (\%) & $4(5.9)$ & $4(7.3)$ & $0(0.0)$ \\
Stopped, no b/csDMARD started, N (\%) & $3(4.4)$ & $3(5.5)$ & $0(0.0)$ \\
Stopped, no b/csDMARD started, with less & $0(0.0)$ & $0(0.0)$ & $0(0.0)$ \\
than 6 months follow up data, N (\%) & & & $0(0.0)$ \\
Missing data, N (\%) & $1(1.5)$ & $1(1.8)$ & \\
\hline
\end{tabular}

Follow-up treatment regimen the first six months after the monotherapy csDMARD treatment failure date, in all non-censored cases with "inefficacy" as main treatment failure cause. The total cohort $(n=68)$ included 1 patient treated with leflunomide (data not shown separately). The rows "continued" refer to the patients in whom the first-line csDMARD monotherapy was continued and an additional DMARD was prescribed as combination therapy. The rows "stopped" refer to the patients in which the first-line CSDMARD was stopped entirely. The follow-up treatment or lack thereof within a window of six months after monotherapy treatment failure date was recorded for non-censored cases.

\section{Supplementary Table S8. Follow-up treatment regimen upon side-effects}

\begin{tabular}{llll}
\hline & All. N = 37 & MTX. N = 31 & SSZ. N = 4 \\
\hline Continued, different csDMARD added, N (\%) & $1(2.7)$ & $1(3.2)$ & $0(0.0)$ \\
Continued, bDMARD added, N (\%) & $0(0.0)$ & $0(0.0)$ & $0(0.0)$ \\
Stopped, different csDMARD started, N (\%) & $18(48.6)$ & $16(51.6)$ & $2(50.0)$ \\
Stopped, bDMARD started, N (\%) & $4(10.8)$ & $3(9.7)$ & $1(25.0)$ \\
Stopped, no b/csDMARD started, N (\%) & $12(32.4)$ & $9(29.0)$ & $1(25.0)$ \\
Stopped, no b/csDMARD started, with less than & $1(2.7)$ & $1(3.2)$ & $0(0.0)$ \\
6 months follow up data, N (\%) & & & \\
Other, N (\%) & $1(2.7)$ & $1(3.2)$ & $0(0.0)$ \\
\hline
\end{tabular}

Follow-up treatment regimen the first six months after the monotherapy CSDMARD treatment failure date, in all non-censored cases with "side-effects" as main treatment failure cause. The total cohort $(n=37)$ included 2 patients treated with leflunomide (data not shown separately). The rows "continued" refer to the patients in whom the first-line CSDMARD monotherapy was continued and an additional DMARD was prescribed as combination therapy. The rows "stopped" refer to the patients in which the first-line csDMARD was stopped entirely. The follow-up treatment or lack thereof within a window of six months after monotherapy treatment failure date was recorded for non-censored cases. 


$$
4
$$




\title{
CHAPTER 4
}

\section{Enrichment of Activated Group 3 Innate Lymphoid Cells in Psoriatic Arthritis Synovial Fluid}

\begin{abstract}
Authors:
Emmerik F.A. Leijten'1, Tessa S. van Kempen', Marianne Boes', Jocea M.R. Michels-van Amelsfort'

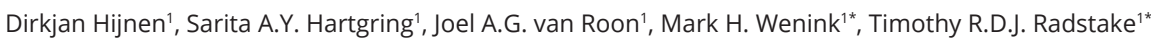
*contributed equally

Affiliation:

1. University Medical Center Utrecht, Utrecht, the Netherlands

Published in:

Arthritis and Rheumatology. 2015 Oct;67(10):2673-8

doi: 10.1002/art.39261
\end{abstract}




\section{Abstract}

Objective: Innate lymphoid cells (ILCS) are a recently discovered group of cells that are essential to epithelial homeostasis and are implicated in psoriasis pathogenesis, yet they have never been reported in psoriatic arthritis (PsA).

Methods: ILC classes and subsets were characterized in the peripheral blood (PB) of healthy controls, patients with psoriasis, and patients with PSA and in the synovial fluid (SF) of patients with PSA and patients with rheumatoid arthritis (RA). Cell surface marker expression and intracellular cytokine production following stimulation were analyzed using flow cytometry.

Results: ILCS were identified in the SF and were 4-fold more abundant in PSA SF than in PSA PB. Fewer CCR6+ ILCS were found in PSA PB than in healthy control PB, while PSA SF was enriched for CCR6+ ILCS compared to PSA PB and RA SF. Natural cytotoxicity receptor NKp44+ group 3 ILCs were rare in PB and RA SF, but abundant in PSA SF. Increased numbers of interleukin-17A (IL-17A)-producing ILCS were present in PSA SF compared to RA SF. CCR6, NKp44, and melanoma cell adhesion molecule (MCAM) were expressed on the cell surface of SF ILCS that produced IL-17A. The number of circulating NKp44+, CCR6+, and MCAM+ ILCs in blood was inversely correlated with PsA disease activity.

Conclusion: Our findings indicate that PSA SF is enriched for group 3 ILCS that express CCR6 and NKp44, which distinguishes the synovial compartment from RA. The increased IL-17A production by SF ILCs indicates a novel role for ILCs in PSA. 


\section{Introduction}

The interleukin-23 (IL-23)/IL-17 cytokine axis is well established in the pathogenesis of both psoriasis and psoriatic arthritis (PsA). The production of IL-17 is mainly considered a T cell phenomenon, although innate lymphoid cells (ILCS) produce it as well. ILCS are a rare immune cell population defined by their lymphoid morphology, lack of T cell receptor, and absence of lineage markers (e.g., markers of dendritic cells, monocytes, stem cells, or mast cells) (1). Conventional natural killer cells are prototypical ILCS, yet more ILCs were recently discovered and are classified into group 1, group 2, or group 3. Based on transcriptional and functional characteristics, these subsets relate to the Th1, Th2, and Th17 T helper cell classifications, respectively (2). For instance, group 1 ILCs and Th1 cells share the transcription factor T-bet and produce interferon- $\gamma$, whereas group 3 ILCs and Th17 cells share the transcription factor retinoic acid receptor-related orphan nuclear receptor $y \mathrm{t}$ and produce IL-17A.

ILCS have gained interest in the field of immunology for their roles in balancing immunity and inflammation in epithelial disease (1). They are an established source of IL-17A in Crohn's disease(3), and an IL-17A-producing subset of ILCS, group 3 ILCS, which express the natural cytotoxicity receptor NKp44, are increased in the skin and blood of patients with psoriasis (4-6). Group 3 ILCs express IL-23 receptor and rely on IL-23 for their full phenotypic development. Although ILCs are a rare subset in peripheral blood (PB), at epithelial sites they are responsible for the first wave of cytokine production and have been shown to be necessary for the development of psoriatic skin lesions $(7,8)$.

We postulate that ILCs migrate not only to skin, but also to the joint, where they may function as rapid innate immune responders that propagate arthritis in PsA. To our knowledge, this is the first study to document the presence, phenotype, and functional capacities of novel ILC groups in the synovial compartment of patients with PsA.

\section{Patients and methods}

\section{Patient population}

PB and synovial fluid (SF) samples were obtained in accordance with local Institutional Review Board approval (Trial ID NTR4626; 13-696-M). All SF samples were collected from the knee joint as part of routine clinical care. Patients with PSA fulfilled Classification of Psoriatic Arthritis Study Group criteria. A rheumatologist (EFAL or JMRM-VA) took the medical history and performed physical examinations of patients with psoriasis in order to exclude concomitant PSA. One subject with PsA donated a matched PB and SF sample; the remainder of samples from patients 
with PsA were unmatched. PB samples were collected from 12 healthy controls (mean \pm SEM age $44 \pm 2.5$ years), 8 patients with psoriasis (age $57 \pm 5.3$ years; disease duration $12 \pm 6.6$ years), and 13 patients with PsA (age $46 \pm 2.8$ years; disease duration $7.7 \pm 2.4$ years). SF samples were collected from 11 patients with PSA (mean \pm SEM age $51 \pm 3.8$ years; mean \pm SEM disease duration $9.5 \pm 3.1$ years) and 12 patients with RA (age $53 \pm 4.2$ years; disease duration $16 \pm 2.6$ years). In patients with PsA, axial involvement was present in 3 patients, enthesitis in 4 patients, and dactylitis in 2 patients. The frequency of erosive disease was lower in the PSA SF group (1 of 11 patients) than in the RA SF group (11 of 12 patients) ( $P$ $<0.05)$. None of the other clinical or demographic features differed between the groups (see Supplementary Table 1, available on the Arthritis \& Rheumatology web site at http://onlinelibrary.wiley.com/doi/10.1002/art.39261/abstract).

\section{Experimental procedure}

PB mononuclear cells and SF mononuclear cells were isolated according to standard Ficoll gradient procedure. Seven million isolated mononuclear cells were stained with the antibody cocktail in the presence of BD Horizon Brilliant stain buffer (catalog no. 563794). ILCs were defined as cells within the lymphocyte gate on the scatter plot that were single cells, lineage negative, CD45 positive, and CD127 positive. See Supplementary Figure 1 for an example of the gating strategy and Supplementary Table 2 (available on the Arthritis \& Rheumatology web site at http://onlinelibrary.wiley.com/doi/10.1002/art.39261/abstract) for the list of antibodies used. ILCs were subsequently classified as group 1 ILCs, group 2 ILCS, or group 3 ILCs based on the markers CD117 (c-Kit) and CD294 (chemoattractant receptor-like molecule expressed on Th2 cells) (Figure 1A). We specifically excluded the population of conventional NKs from our ILC population (using CD94 and CD16, as previously proposed [1]) to focus on the novel subgroups of ILCs, and our data on ILCs should be interpreted as referring to "ILCs minus conventional NKs." In order to detect intracellular cytokine production by ILCS, SF mononuclear cell samples were thawed and stimulated for 4 hours at $37^{\circ} \mathrm{C}$ in culture medium (RPMI 1640 medium [Gibco] with 10\% fetal calf serum, 1\% penicillin/streptomycin, and $1 \%$ glutamine) containing phorbol myristate acetate (PMA; $50 \mathrm{ng} / \mathrm{ml})$, ionomycin ( $1 \mu \mathrm{g} /$ $\mathrm{ml}$ ), and GolgiStop (catalog no. 554724) followed by extracellular and intracellular antibody staining. ILC-specific cytokine production was also compared to the expression of cell surface markers (before and after stimulation). Samples were measured on an LSRFortessa analyzer. Dead cells were excluded using a viability dye. Cutoff values were determined using fluorescence minus one. SF samples were handled and stored the same way, with subsequent experiments being performed pairwise (i.e., PsA samples matched with RA samples) using identical protocols to avoid potential confounders. 


\section{Statistical analysis}

Our primary objective was to compare PB ILCs between healthy controls, patients with psoriasis, and patients with PSA and to compare SF ILCS from patients with PSA to SF ILCs from patients with RA and PB ILCS from patients with PSA. Our secondary objective was to evaluate the correlation between PB ILC classes or subsets and the clinical features of the patients with psoriasis or PsA. Normally distributed data are presented as the mean \pm SEM and were analyzed by Student's t-test and Pearson correlation. Non-normally distributed data are presented as the median (interquartile range [IQR]) and were analyzed by Mann-Whitney $U$ test and Spearman's test. $P$ values less than 0.05 were considered significant. FACSDiva version 7.0 and FlowJo version 10 software were used for fluorescenceactivated cell sorting (FACS) data analysis and FACS graphs. Data were analyzed using GraphPad Prism version 6.

\section{Results}

\section{Composition of ILC classes in PB and SF}

Patients and healthy controls had comparable frequencies of ILCs in PB, which was $\sim 0.1 \%$ of circulating lymphocytes. Subdividing PB ILCs into group 1 ILCs, group 2 ILCs, or group 3 ILCs resulted in an 1:1:1 ratio (Figure 1A), without skewing in patients versus controls (Figures 1B-D). We readily identified ILCS in SF samples using the same gating strategy used to identify PB ILCs (see Supplementary Figure 1, available on the Arthritis \& Rheumatology web site at http://onlinelibrary.wiley. com/doi/10.1002/art.39261/abstract). Moreover, ILCs were 4-fold more abundant in PSA SF (mean \pm SEM $0.55 \pm 0.14 \%)$ than in PSA PB $(0.12 \pm 0.015 \%)(P<0.05)$ (Figure 1B). There was a trend toward a greater percentage of ILCS in PSA SF than in RA SF $(0.27 \pm 0.11 \%)$, but this difference did not reach significance $(P=0.13)$. Compared to PB, group 1 ILCs were expanded in the SF of both PSA and RA (Figure 1B) $(P<0.05)$, whereas group 3 ILCS were specifically expanded in PSA SF (38 \pm $6.6 \%)$ compared to RA SF $(13 \pm 2.7 \%)(P<0.05)$. Group 2 was the least common class of ILCS found in SF, yet group 2 ILCS were more common in PSA SF $(9.2 \pm$ $2.9 \%)$ than in RA SF $(1.9 \pm 0.89 \%)(P<0.05)$ (Figures 1B-D). 
A

\section{Classification of ILCs}

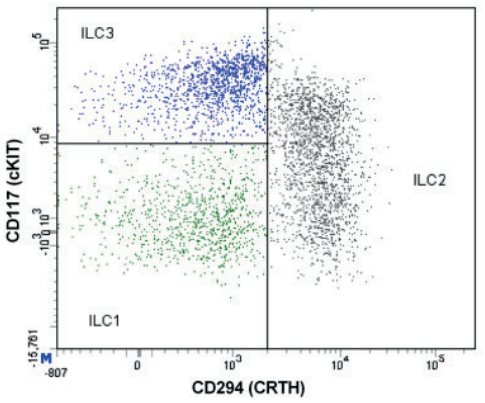

C

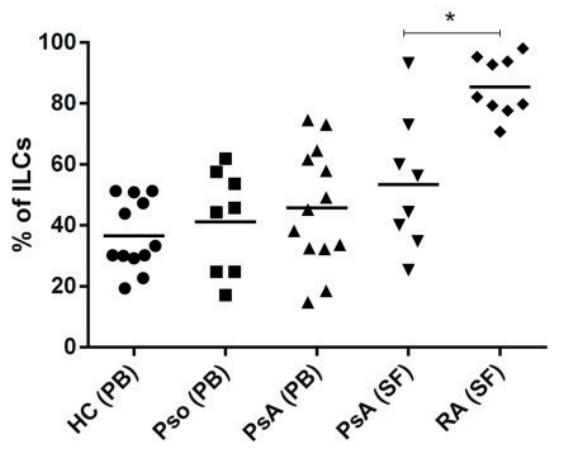

B

Frequency of ILCs

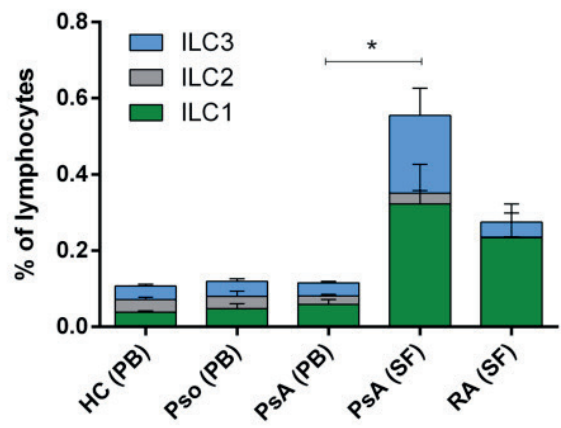

D

Group 3 ILCs

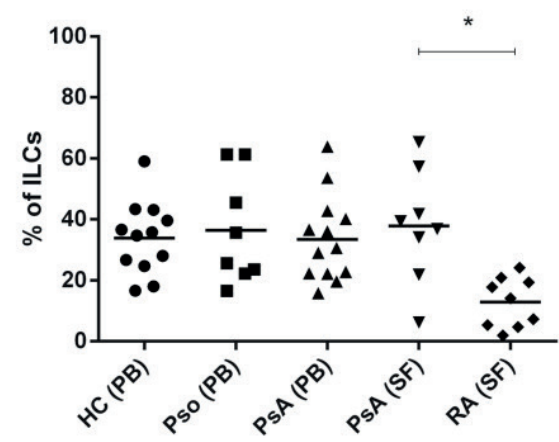

Figure 1

Increased abundance of innate lymphoid cells (ILCS) in psoriatic arthritis (PSA) synovial fluid (SF). A, A representative fluorescence-activated cell sorter plot of a PsA peripheral blood (PB) sample, demonstrating the gating strategy that classified ILCS as either group 1 ILCS (ILC1) (CD117-CD294-), group 2 ILCS (CD294+), or group 3 ILCS (CD117+CD294-). B, Frequency of groups 1, 2, and 3 ILCs in PB from healthy controls (HCS), patients with psoriasis (Pso), and patients with PsA and in SF from patients with PSA and patients with rheumatoid arthritis (RA), presented as a percentage of the total number of lymphocyte events measured. Bars show the mean \pm SEM. C and D, Distribution of ILC classes as a percentage of the entire ILC population. Symbols represent individual patients; horizontal lines show the median. $*=P<0.05$. 


\section{Activation and homing markers on ILCs in PB and SF}

The percentages of CCR6+ ILCS in the PB of patients with PSA (median 24\% [IQR 20-32\%]) were reduced compared to healthy controls (median 41\% [IQR 31-45\%]) $(P<0.05)$. A greater percentage of CCR6+ ILCS was found in PSA SF (median $45 \%$ [IQR 35-62\%]) than in PSA PB or RA SF (median 22\% [IQR 6.9-32\%]) (both $P<0.05$ ) (Figure 2A). NKp44+ group 3 ILCs were rare in PB overall but were more common in the PB of patients with psoriasis (0.90\% [IQR 0.30-1.4\%]) and the PB of patients with PSA $(0.50 \%$ [IQR $0.40-1.2 \%])$ than in the PB of healthy controls $(0.20 \%$ [IQR $0.10-0.40 \%]$ ) (both $P<0.05)$. In contrast, NKp44+ group 3 ILCs represented a large proportion of SF ILCS, specifically in PSA SF (12\% [IQR 3.0-19\%]), but not RA SF (0.99\% [IQR 0.29-2.4\%]) $(P<0.05)$ (Figure 2B). NKp44+ group 3 ILCs uniformly coexpressed CCR6. Analysis of one matched, freshly isolated sample of PB and SF from a patient with PSA showed a shift toward a predominant CCR6+ ILC population in SF, of which a robust proportion express NKp44 (Figure 2C). When examining other activation markers, we found that ILCs in the SF also expressed more melanoma cell adhesion molecule (MCAM) and CD56, but these markers did not differentiate PSA SF from RA SF (see Supplementary Figure 2, available on the Arthritis \& Rheumatology web site at http://onlinelibrary.wiley.com/doi/10.1002/ art.39261/abstract). However, CCR6 was coexpressed on the CD56+ and MCAM+ ILCS specifically in PSA SF (see Supplementary Figure 3, available on the Arthritis \& Rheumatology web site at http://onlinelibrary.wiley.com/doi/10.1002/art.39261/ abstract). There were no differences between groups in PB ILC expression of CXCR3, HLA-DR, or common leukocyte antigen. 
A

CCR6+ ILCs

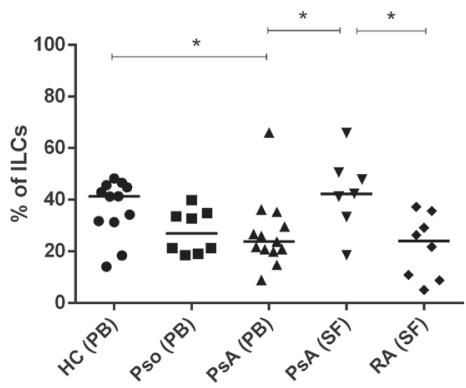

B

$\mathrm{NCR}+$ ILC3

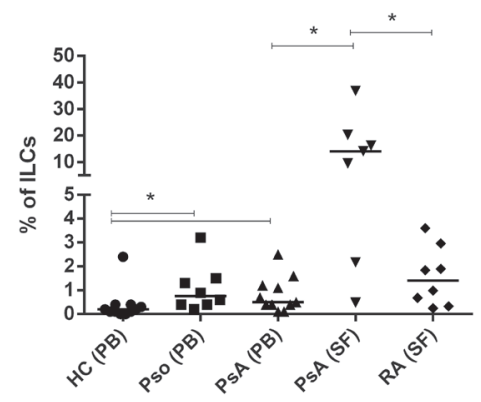

C

PsA PB

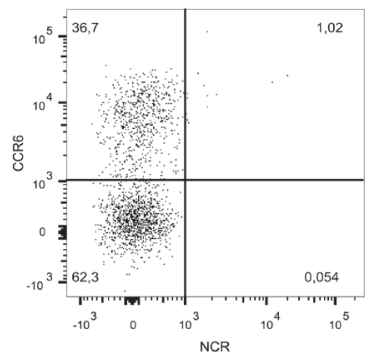

PsA SF

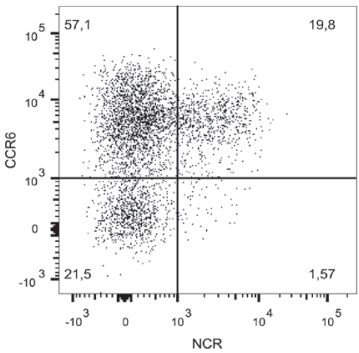

RA SF

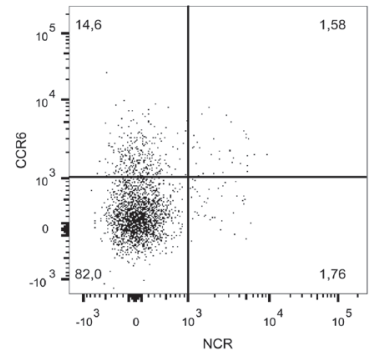

\section{Figure 2}

Increased presence of CCR6- and NKp44-expressing innate lymphoid cells (ILCS) in psoriatic arthritis (PSA) synovial fluid (SF). A and B, Levels of CCR6+ ILCS (A) and NKp44+ group 3 ILCS (ILC3) (B), expressed as a percentage of the total number of ILC events measured. Symbols represent individual patients; horizontal lines show the median. $*=P<0.05$. HC = healthy control; Pso = psoriasis. C, Matched peripheral blood (PB) and SF from a patient with PSA, showing that PSA PB contains relatively few CCR6+ ILCs and that NKp44+ ILCS are very scarce in PSA PB, whereas PSA SF is enriched for CCR6+ ILCS and contains a clear NKp44+ (CCR6+) ILC population. A representative rheumatoid arthritis (RA) SF sample is shown to illustrate that CCR6 and NKp44 expression was specific for PSA SF. 


\section{Production of IL-17A by SF ILCs and relationship of PB ILCs to disease activity}

Upon in vitro stimulation of SF ILCS, IL-17A production by SF ILCS was observed (see Supplementary Figure 4, available on the Arthritis \& Rheumatology web site at http://onlinelibrary.wiley.com/doi/10.1002/art.39261/abstract). Increased percentages of IL-17A-producing SF ILCS were observed in PSA (median 2.4\% [IQR 1.3-2.8\%]) compared to RA (median 0.77\% [IQR 0.40-1.2\%]) $(P<0.05)$ (Figure 3A). There was no difference in IL-22-producing ILCS in PSA SF (median $0.70 \%$ [IQR $0.28-1.7 \%]$ ) versus RA SF (1.4\% [IQR $0.30-3.2 \%]$ ). IL-17A-producing ILCS coexpressed CCR6, NKp44, and MCAM, but did not coexpress CD56 (Figure 3B). Consistent with these findings, the frequency of IL-17A+ ILCS after stimulation was also correlated with the frequency of CCR6+ ILCS ( $r=0.68)$, NKp44+ group 3 ILCS $(r=0.82)$, and MCAM+ ILCs $(r=0.59)$ present in the SF prior to stimulation. There was no cell surface marker that could specifically identify all IL-17A+ ILCs. Upon in vitro stimulation of PB ILCS, no production of IL-17A or IL-22 was observed.

Interestingly, high disease activity was correlated with reduced frequencies of PB ILCs classified as CCR6+ group 3 ILCS ( $r=-0.63$ when disease activity was measured by erythrocyte sedimentation rate [ESR]) (Figure 3C), NKp44+ $(r=-0.81$ when disease activity was measured by Disease Activity Score in 28 joints using the C-reactive protein level [DAS28-CRP]) (Figure 3D), and MCAM+ $(r=-0.79$ when disease activity was measured by DAS28-CRP and $r=-0.90$ when disease activity was measured by Disease Activity for Psoriatic Arthritis) $(P<0.05)$. No correlation between other ILC subsets and disease activity or other clinical features of disease was observed. 
A

IL-17A+

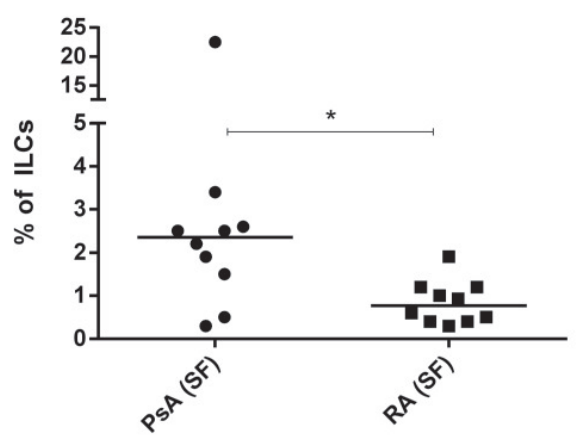

C

CCR6+ ILC3

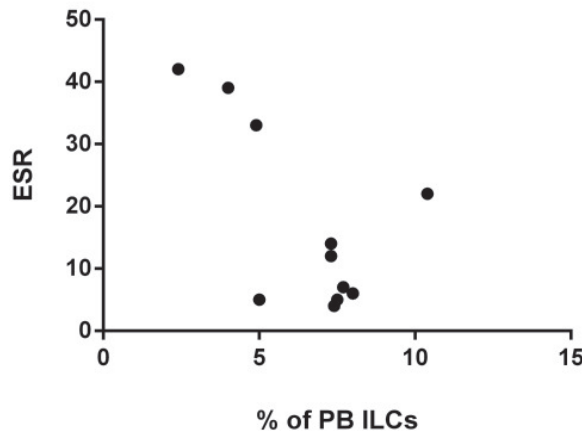

B
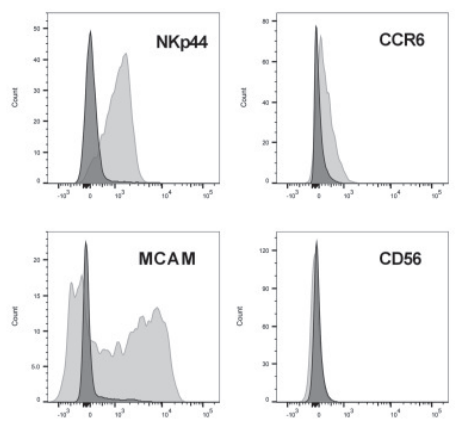

D

NKp44+ ILCs

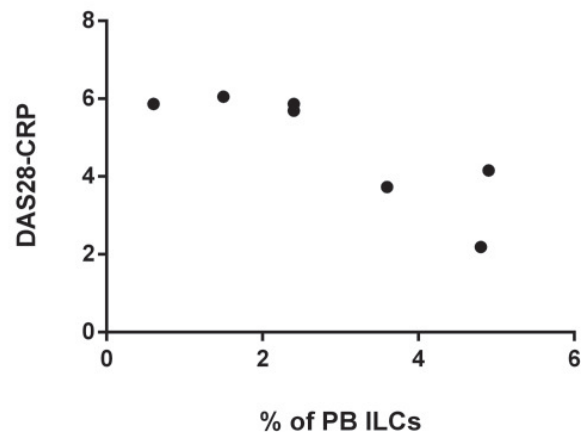

\section{Figure 3}

Increased levels of interleukin-17A (IL-17A)-producing innate lymphoid cells (ILCS) in psoriatic arthritis (PsA) synovial fluid (SF) and inverse relationship between IL-17A-producing subsets in peripheral blood (PB) and disease activity. A, Levels of IL-17A+ ILCS in PsA and rheumatoid arthritis (RA) SF mononuclear cells that were thawed and stimulated for 4 hours in the presence of phorbol myristate acetate/ ionomycin/GolgiStop. The percentage of ILCS that stained positive for IL-17A is shown as a percentage of the total number of ILC events measured. Symbols represent individual patients; horizontal lines show the median. $*=P<0.05$. B, Expression of NKp44, CCR6, melanoma cell adhesion molecule (MCAM), and CD56 in IL-17A+ SF ILCS and IL-17A- SF ILCs from a representative PSA SF sample. C, Inverse relationship between the erythrocyte sedimentation rate (ESR) in patients with PSA and the percentage of PB ILCS classified as CCR6+ group 3 ILCS (ILC3) ( $r=-0.63, P<0.05)$. D, Inverse relationship between the Disease Activity Score in 28 joints using the C-reactive protein level (DAS28-CRP) in patients with PsA and the percentage of PB ILCs classified as NKp44+ $(r=-0.81, P<0.05)$. 


\section{Discussion}

This is the first study to systematically characterize all 3 groups of ILCs in SF and PB and compare 2 different rheumatic diseases. Recent reports indicate that NKp44+ group 3 ILCs are involved in the development of psoriasis. Our study establishes that NKp44+ group 3 ILCs also accumulate in the joints of patients with PsA.

ILCs were significantly increased in PSA SF compared to PB, which is similar to increased numbers of ILCS in psoriatic skin compared to blood $(4,5)$. Our ILC data are best interpreted as the net effect of ILC differentiation, combined with influx/efflux within the compartment analyzed (SF or PB). Environmental factors are known to steer the developmental fate of ILCS, and plasticity between classes has been reported. Of relevance to our results, the presence of IL-1 $\beta$ and IL-23 can direct ILCS from the NKp44- phenotype toward the NKp44+ phenotype, at least under culture conditions $(4,9,10)$. The actual presence of and genetic susceptibility to such a milieu might explain why NKp44+ group 3 ILCs were specifically abundant in PSA SF, but not in RA SF. The present study is exploratory in nature and included relatively few subjects, which restricts interpretation regarding a causal relationship between specific SF ILCS and disease pathogenesis or clinical phenotype. However, a recent study confirmed the presence of NKp44+ ILCs in the SF of patients with ankylosing spondylitis, a disease similarly marked by a strong IL-23/IL-17 pathway (11).

Interestingly, patients with PSA had fewer CCR6+ ILCS in PB. Reciprocally, CCR6 was up-regulated on SF ILCS from patients with PSA, which suggests that this chemokine receptor may contribute to the extravasation of ILCs into the joints of patients with PSA. Our group has previously identified an increase in CCL20, the CCR6 ligand, in PSA SF (see Supplementary Figure 5, available on the Arthritis \& Rheumatology web site at http://online library.wiley.com/doi/10.1002/art.39261/ abstract), but functional studies are needed to determine whether ILC homing to the joint is CCL20/CCR6 mediated. ILCs from the SF of patients with PSA also expressed high levels of MCAM, an integrin linked to IL-17A-producing T cells and expressed in ILCs(12), and high levels of CD56, which is associated with the effector phenotype of ILCS (13).

We showed that IL-17A is produced by ILCs in the joints of patients with PSA and is produced by more than one ILC subset. Our results indicate that the "Th17-like" label of group 3 ILCs is an oversimplification and are consistent not only with what is already known in T cells, but also with the findings of a recent study on the transcriptome of different ILC classes, which found that IL-17A was not group 3 ILC restricted (14). SF samples were obtained at a stage in the disease when other cells have massively homed to the inflamed joint, thus when the absolute contribution of ILC-produced cytokines is relatively low. However, the established function 
of ILCS is their rapid (innate) production of cytokines prior to the production by T cells, and our results point to ILCS as candidate cells capable of initiating an inflammatory cascade in the joint.

Beyond the cytokine effector function of ILCs, little is known about their function in humans, and future studies should evaluate the interaction between ILCS and other cells in the joint (13). It is unclear if the presence of NKp44 on SF ILCs only signifies an activated status or if this receptor itself induces activation of ILCS (13, 15). Tonsil-derived NKp44+ ILCs have been shown to produce tumor necrosis factor following activation of their NKp44 receptor (15). In addition, conventional NKs also express NKp44, which recognizes the NKp44 ligand on normal human chondrocytes (16) and fibroblast-like synoviocytes (17) to directly cause (cytotoxic) activity. It will be of interest to determine whether these ligands can similarly activate NKp44+ SF ILCS in the joint early in the disease course.

We conclude that distinct classes of ILCS are present in the joints of patients with rheumatic diseases. Group 1 ILCs are predominant in RA SF, whereas PsA SF is characterized by CCR6- and NKp44-expressing group 3 ILCS with increased capacity to produce IL-17A. These findings underscore the critical role played by the IL-23/IL-17 pathway in PSA and ILCS as potential instigators of inflammation in the joints of patients with PsA. 


\section{References}

1. Hazenberg MD, Spits H. Human innate lymphoid cells. Blood 2014;124:700-9.

2. Spits H, Artis D, Colonna M, Diefenbach A, Di Santo JP, Eberl G, et al. Innate lymphoid cells-a proposal for uniform nomenclature. Nat Rev Immunol 2013;13:145-9.

3. Geremia A, Arancibia-Carcamo CV, Fleming MP, Rust N, Singh B, Mortensen NJ, et al. IL-23responsive innate lymphoid cells are increased in inflammatory bowel disease. J Exp Med 2011;208:1127-33.

4. Teunissen MB, Munneke JM, Bernink JH, Spuls PI, Res PC, Te Velde A, et al. Composition of innate lymphoid cell subsets in the human skin: enrichment of NCR+ ILC3 in lesional skin and blood of psoriasis patients. J Invest Dermatol 2014;134:2351-60.

5. Villanova F, Flutter B, Tosi I, Grys K, Sreeneebus H, Perera GK, et al. Characterization of innate lymphoid cells in human skin and blood demonstrates increase of NKp44+ ILC3 in psoriasis. The J Invest Dermatol 2014;134:984-91.

6. Dyring-Andersen B, Geisler C, Agerbeck C, Lauritsen JP, Gudjonsdottir SD, Skov L, et al. Increased number and frequency of group 3 innate lymphoid cells in nonlesional psoriatic skin. Br J Dermatol 2014;170:609-16.

7. Basu R, O'Quinn DB, Silberger DJ, Schoeb TR, Fouser L, Ouyang W, et al. Th22 cells are an important source of IL-22 for host protection against enteropathogenic bacteria. Immunity 2012;37:1061-75.

8. Pantelyushin S, Haak S, Ingold B, Kulig P, Heppner FL, Navarini AA, et al. Rorgammat1 innate lymphocytes and gD T cells initiate psoriasiform plaque formation in mice. J Clin Invest 2012;122:2252-6.

9. Cella M, Otero K, Colonna M. Expansion of human NK-22 cells with IL-7, IL-2, and IL-1b reveals intrinsic functional plasticity. Proc Natl Acad Sci U S A 2010;107:10961-6.

10. Bernink JH, Peters CP, Munneke M, te Velde AA, Meijer SL, Weijer K, et al. Human type 1 innate lymphoid cells accumulate in inflamed mucosal tissues. Nat Immunol 2013;14:221-9.

11. Ciccia F, Guggino G, Rizzo A, Saieva L, Peralta S, Giardina A, et al. Type 3 innate lymphoid cells producing IL-17 and IL-22 are expanded in the gut, in the peripheral blood, synovial fluid and bone marrow of patients with ankylosing spondylitis. Ann Rheum Dis 2015. E-pub ahead of print.

12. Montaldo E, Teixeira-Alves LG, Glatzer T, Durek $P$, Stervbo $U$, Hamann $W$, et al. Human RORgt(+)CD34(+) cells are lineagespecified progenitors of group 3 RORgt(+) innate lymphoid cells. Immunity 2014;41:988-1000.

13. Mizuno S, Mikami Y, Kamada N, Handa T, Hayashi A, Sato T, et al. Cross-talk between RORgt+ innate lymphoid cells and intestinal macrophages induces mucosal IL-22 production in Crohn's disease. Inflamm Bowel Dis 2014;20:1426-34.

14. Robinette ML, Fuchs A, Cortez VS, Lee JS, Wang Y, Durum SK, et al. Transcriptional programs define molecular characteristics of innate lymphoid cell classes and subsets. Nat Immunol 2015;16:306-17. 
15. Glatzer T, Killig M, Meisig J, Ommert I, Luetke-Eversloh $M$, Babic $M$, et al. RORgt(+) innate lymphoid cells acquire a proinflammatory program upon engagement of the activating receptor NKp44. Immunity 2013;38:1223-35.

16. Bialoszewska A, Baychelier F, Niderla-Bielinska J, Czop A, Debre P, Vieillard V, et al. Constitutive expression of ligand for natural killer cell NKp44 receptor (NKp44L) by normal human articular chondrocytes. Cell Immunol 2013;285:6-9.

17. Nielsen N, Pascal V, Fasth AE, Sundstrom $Y$, Galsgaard ED, Ahern D, et al. Balance between activating NKG2D, DNAM-1, NKp44 and NKp46 and inhibitory CD94/NKG2A receptors determine natural killer degranulation towards rheumatoid arthritis synovial fibroblasts. Immunol 2014;142:581-93. 


\section{Supplementary information}

Table 1: Demographics and disease activity of study participants

\begin{tabular}{lccccc}
\hline & HC PB & Pso PB & PsA PB & PsA SF & RA SF \\
\hline Samples & 12 & 8 & 13 & 11 & 12 \\
Total samples $(\mathrm{n})$ & 12 & 6 & 13 & 2 & 3 \\
Fresh samples $(\mathrm{n})$ & 0 & 2 & 0 & 9 & 9
\end{tabular}

Demographics

Age (y)
Female
Pso duration (y)
PsA/RA duration (y)

$\begin{array}{ccccc}44 \pm 2.5 & 57 \pm 5.3 & 46 \pm 2.8 & 51 \pm 3.8 & 53 \pm 4.2 \\ 9 / 12 & 4 / 8 & 5 / 13 & 5 / 11 & 4 / 12 \\ . & 12 \pm 6.6 & 20 \pm 4.8 & 12 \pm 4.6 & . \\ . & . & 7.7 \pm 2.4 & 9.5 \pm 3.1 & 16 \pm 2.6\end{array}$

Therapy

Prednisone (oral)
Methotrexate
TNF.inhibitor

$\begin{array}{llll}0 / 8 & 0 / 13 & 2 / 11 & 6 / 12 \\ 1 / 8 & 5 / 13 & 6 / 11 & 5 / 12 \\ 0 / 8 & 0 / 13 & 0 / 11 & 2 / 12\end{array}$

Disease activity

\begin{tabular}{|c|c|c|c|c|}
\hline PASI & $3.3(1.2-6.1)$ & $2.1(0.75-2.9)$ & $1.4(0.3-2.4)$ & . \\
\hline $28 \mathrm{TJC}(\mathrm{n})$ & . & $5.5 \pm 1.4$ & $2.8 \pm 0.75$ & $6.0 \pm 3.0$ \\
\hline $28 \mathrm{SJC}(\mathrm{n})$ & . & $5.3 \pm 1.5$ & $3.0 \pm 0.91$ & $5.6 \pm 2.6$ \\
\hline $\operatorname{ESR}(\mathrm{mm} / \mathrm{hr})$ & $8.5(3.5-15)$ & $7(4.5-28)$ & $\mathrm{m}$ & $\mathrm{m}$ \\
\hline CRP (mg/L) & $3.9 \pm 1.9$ & $10 \pm 2.7$ & $\mathrm{~m}$ & $\mathrm{~m}$ \\
\hline DAS28-ESR score & . & $4.1 \pm 0.61$ & $\mathrm{~m}$ & $\mathrm{~m}$ \\
\hline DAS28-CRP score & . & $4.2 \pm 0.57$ & $\mathrm{~m}$ & $\mathrm{~m}$ \\
\hline DAPSA score & . & $39 \pm 8.5$ & $\mathrm{~m}$ & . \\
\hline Dactylitis present & . & $1 / 13$ & $1 / 11$ & . \\
\hline Axial involvement & . & $3 / 13$ & $0 / 11$ & . \\
\hline Enthesitis present & . & $4 / 13$ & $\mathrm{~m}$ & . \\
\hline Erosive disease & . & $6 / 13$ & $1 / 11$ & $11 / 12 *$ \\
\hline RF/aCCP positive & . & $0 / 13$ & $0 / 11$ & $10 / 12$ \\
\hline
\end{tabular}

Table 1: Data shown as mean \pm SEM or median (IQR). One patient with PSA had donated a matched PB and SFsample, the remainder of samples were unmatched. There were significantly more patients with erosive disease in the RA SF group compared to the PSA SF group (fishers exact test, $\mathrm{p}<0.05$ ). No other variables differed between the study groups. SF samples were collected during synovial fluid aspiration as part of standard care, when other disease activity parameters were typically not assessed by the treating physician resulting in some missing clinical data $(\mathrm{m})$ in the SF groups. 
Table 2: list of antibodies used

\begin{tabular}{|c|c|c|c|c|}
\hline Antibody & Description & Fluorochrome & Company & Catalog \# \\
\hline CD3 & Lineage: T-cell & FITC & BD Horizon & 345763 \\
\hline CD19 & Lineage: B-cell & FITC & BD Horizon & 555412 \\
\hline CD14 & Lineage: Monocyte & FITC & BD Horizon & 555397 \\
\hline CD16 & Lineage: NK & FITC & BD Horizon & 555406 \\
\hline CD94 & Lineage: NK & FITC & eBioscience & $11-0949-41$ \\
\hline CD11c & Lineage: $\mathrm{mDC}$ & FITC & Biolegend & 337214 \\
\hline CD141 & Lineage: $\mathrm{mDC}$ & FITC & Miltenyi & $130-090-513$ \\
\hline CD34 & Lineage: stem cell & FITC & Biolegend & 343603 \\
\hline BDCA2 & Lineage: pDC & FITC & Miltenyi & $130-090-51$ \\
\hline CD123 & Lineage: pDC & FITC & eBioscience & $11-1239-42$ \\
\hline FCeR1 & Lineage: mast cell & FITC & eBioscience & $11-5899-41$ \\
\hline CD127 & IL-7Ra & AF647 & Biolegend & 351318 \\
\hline CD161 & KLRB1 & BV510 & Biolegend & 339921 \\
\hline CD45 & Leucocytes & PE-Cy7 & BD Horizon & 557748 \\
\hline CD294 & CRTH2 & PE-CF594 & BD Horizon & 563501 \\
\hline CD117 & CKIT & BV605 & Biolegend & 313217 \\
\hline CD336 & NKp44 & PerCP-eF710 & eBioscience & $46-3369-41$ \\
\hline CD56 & NCAM & AF700 & BD Horizon & 557919 \\
\hline CD146 & MCAM & BV711 & BD Horizon & 563186 \\
\hline CD196 & CCR6 & PE & BD Horizon & 559562 \\
\hline CD196 & CCR6 & PerCP-Cy5.5 & BD Horizon & 560467 \\
\hline HLA-DR & $\mathrm{MHC} \|$ & BV711 & BD Horizon & 563696 \\
\hline CD183 & CXCR3 & PB & Biolegend & 353723 \\
\hline CLA & Skin homing & PB & Biolegend & 321307 \\
\hline IL-22 & IL-22 & eF450 & eBioscience & $48-7229-41$ \\
\hline IL-17A & IL-17A & $\mathrm{PE}$ & eBioscience & $12-7179-42$ \\
\hline Viability Dye & Live cells & eF506 & eBioscience & $65-0866-14$ \\
\hline
\end{tabular}



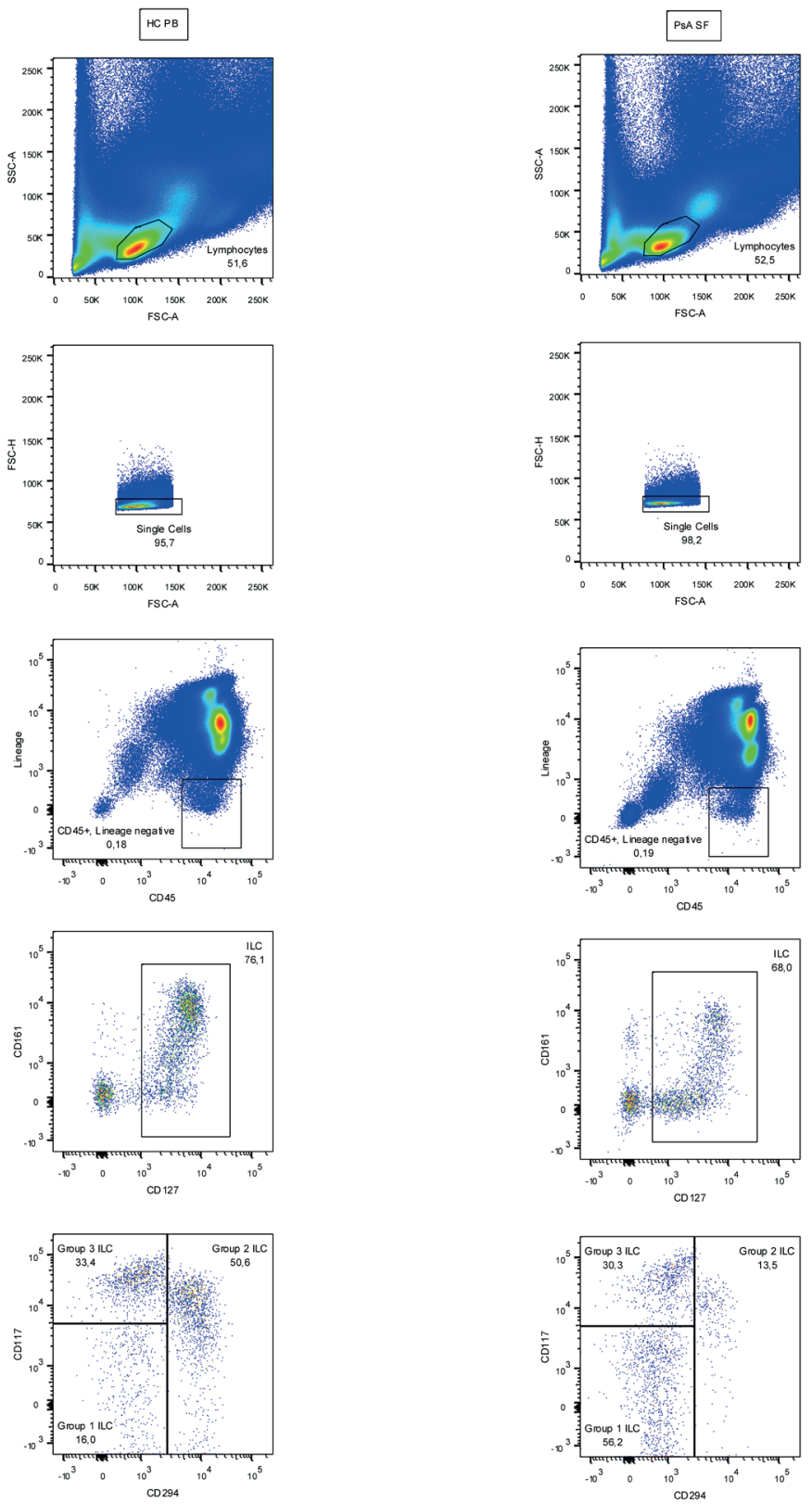

Supplementary figure 1: ILC gating strategy. Representative gating strategy for analysis of ILCs from freshly isolated PBMC (HC) and SFMC (PSA) sample is shown. ILCS were defined as falling within the lymphocyte gate, singles, lineage negative, CD45+, CD127+ (as expected, CD161 was not uniformly expressed by ILCS). ILC1 are identified as CD117- and CD294-; ILC2 as CD294+; ILC3 as CD117+ and CD294- 
$M C A M+I L C S$

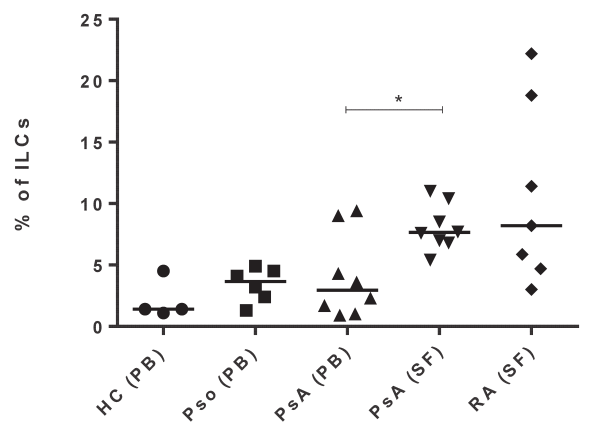

CD $56+$ ILCS

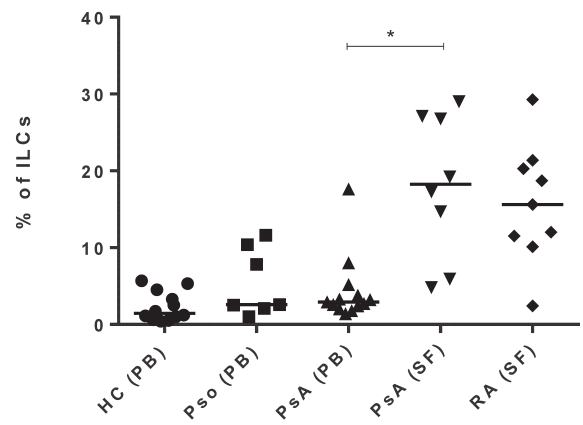

Supplementary figure 2: Increased intra-articular frequencies of MCAM-expressing and CD56expressing ILCS. (A) In PB, there was no difference in MCAM expression between HC, Pso, and PsA. In PSA SF, increased percentages of ILCS expressing MCAM as compared to PSA PB were observed $(p<0.05)$. There was no difference in the expression of MCAM between PSA SF and RA SF. (B) In PB, there was no difference in CD56 expression between HC, Pso, and PSA. In PSA SF, there were more ILCs expressing CD56 than in PSA PB $(p<0.05)$. There was no difference in the expression of CD56 between PSA SF and RA SF. Bars represent the median per group. * indicates $p<0.05$ 

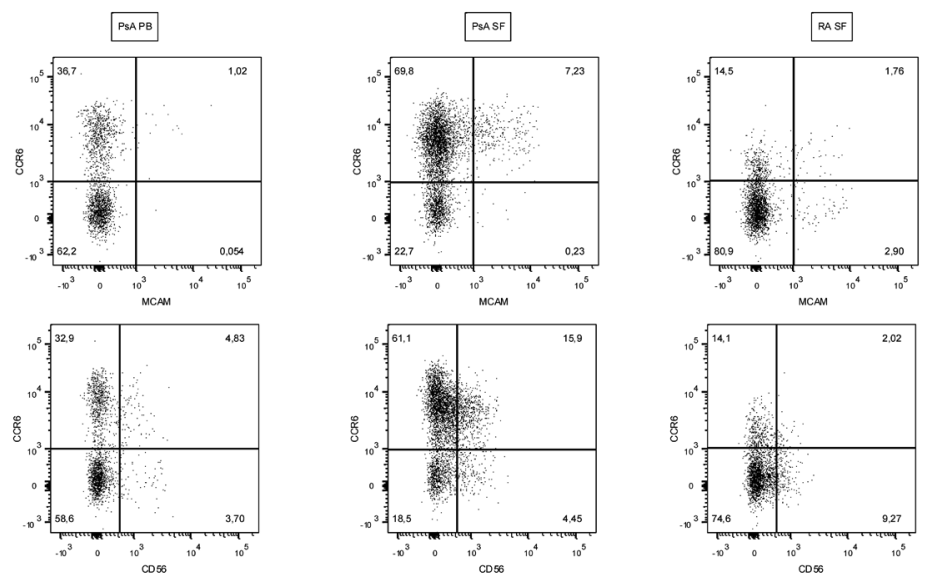

B
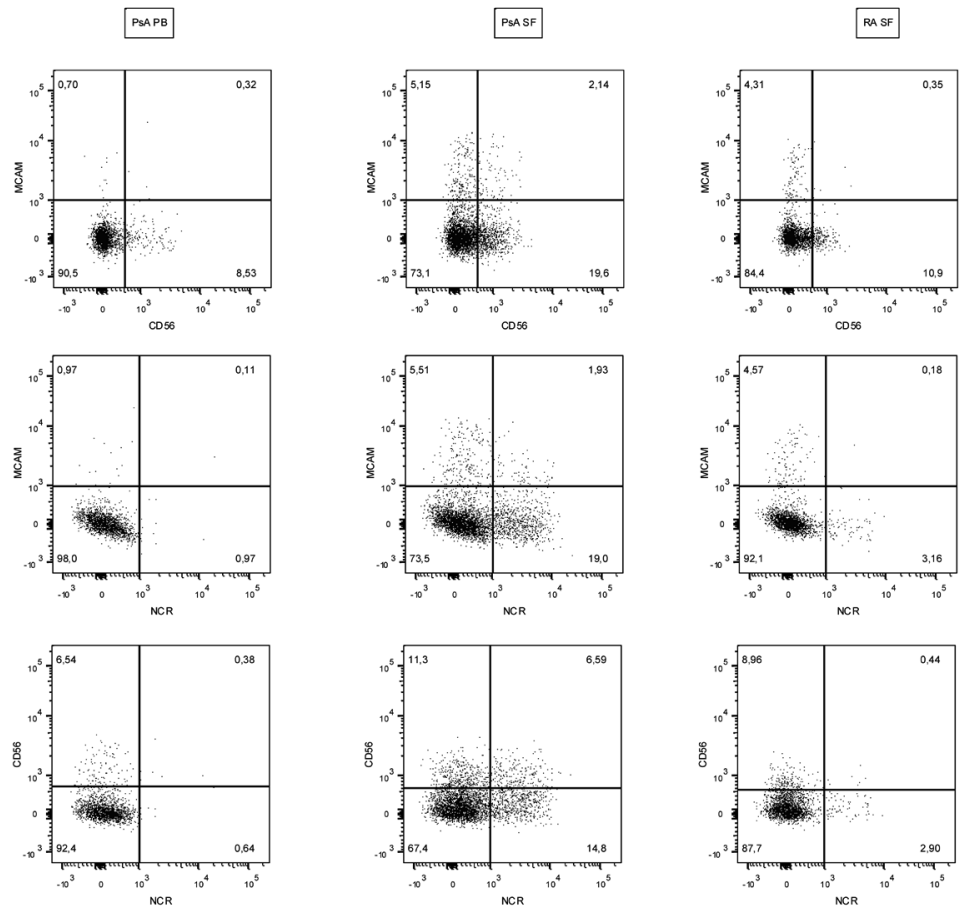

Supplementary figure 3: Representative MCAM and CD56 expression in PSA PB, PSA SF, and RA SF. (A) MCAM+ ILCS and CD56+ ILCS in PSA SF typically co-expressed CCR6, whereas this was not the case for MCAM+ or CD56+ ILCS in RA SF. (B) The co-expression pattern of NKp44 (NCR), MCAM, and CD56 is shown. As can be appreciated, NKp44+, MCAM+, and CD56+ ILCs are distinct ILC subsets: not typically co-expressed on the same ILC. 
PSA SF

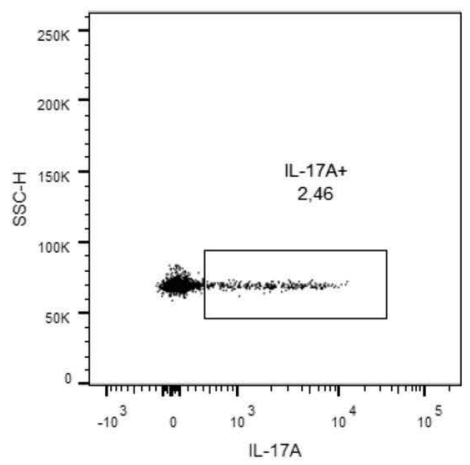

FMO SF

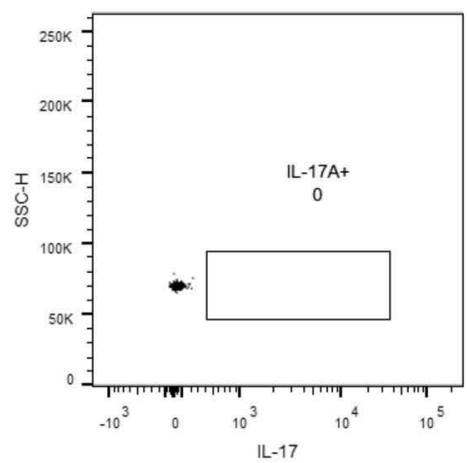

Supplementary figure 4: The gating strategy to identify IL-17A+ ILCS, based on the corresponding negative control of that sample (fluorescence minus one, 'FMO'). Stimulated PB ILCS did not produce IL17A or IL-22, neither in patients or healthy controls (not shown).

CCL20 in SF

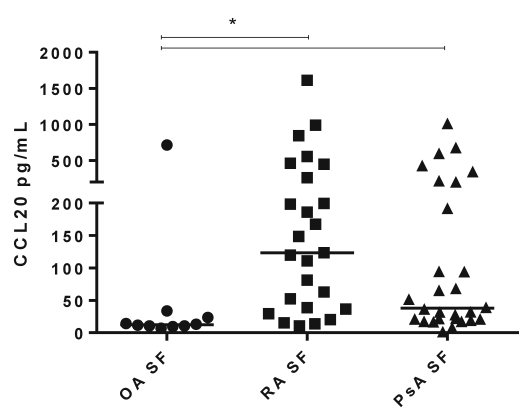

Supplementary figure 5: Increased CCL20 concentrations in joints of patients with RA and PSA. In a previous cohort we have quantified the concentration of CCL20, the known ligand of CCR6, in SF samples collected from patients with osteoarthritis (OA, $n=10)$, rheumatoid arthritis $(R A, n=25)$, and psoriatic arthritis (PsA, n=28). Both RA patients and PSA patients had elevated levels of CCL20 in SF compared to OA, without a difference between RA and PsA. The bars represent median; * $p<0.05$, Mann-Whitney test. 
Innate lymphoid cells in psoriatic arthritis synovial fluid 


$$
5
$$




\section{CHAPTER 5}

\section{Innate lymphoid cells: sparking inflammatory rheumatic disease?}

Authors:

Mark H. Wenink ${ }^{1 *}$, Emmerik F.A. Leijten ${ }^{2 *}$, Tom Cupedo ${ }^{3}$, Timothy R.D.J. Radstake ${ }^{2}$ *authors contributed equally

Affiliation:

1. Sint Maartenskliniek, Nijmegen, The Netherlands

2. University Medical Centre Utrecht, Utrecht, The Netherlands 3. Erasmus University Medical Center, Rotterdam, The Netherlands

Published in:

Arthritis and Rheumatology. 2017 May;69(5):885-897

doi: $10.1002 /$ art.40068 


\section{Introduction}

Immunology research is in a constant state of flux with new cell types being described on a regular basis. While most rheumatologists are familiar with the role that "T cell cytokines" such as tumor necrosis factor (TNF), interferon-y (IFNY), and interleukin-17A (IL-17A) play in the pathogenesis of rheumatic diseases, what many may not know is that an array of innate counterparts to the conventional T cells have recently been discovered that are capable of rapidly producing these same effector cytokines. These innate counterparts include $\gamma / \delta T$ cells, invariant natural killer (iNK) cells, mucosa-associated invariant T (MAIT) cells, and innate lymphoid cells (ILCS). An insightful overview of $\gamma / \delta T$ cells, iNK cells, and MAIT cells in rheumatic disease has recently been published (1); therefore, the current review focuses on ILCs. Strikingly, some of the ILCs have only recently been discovered, in part due to their relative scarcity in peripheral blood, and their presence in numerous organs of the human body has subsequently been described. In this review, we provide the newest insights into the potential role of ILCs in the field of rheumatology and provide recommendations for future ILC research that will help to promote our understanding of rheumatic diseases even further.

\section{Essential background on recently discovered ILCs}

\section{ILCs as innate counterparts to T cells}

Considerable phenotypic and functional overlap exists between ILCS and Th cells, yet there are important differences in activation pathways and tissue localization enabling distinct roles in inflammation. The priming of Th cells requires the recognition of peptide-loaded major histocompatibility complex (MHC) class II molecules provided by professional antigen-presenting cells such as dendritic cells (DCs). In contrast, ILCs do not express rearranged antigen receptors capable of recognizing antigens in a major histocompatibility complex. Activation of ILCS is instead mediated by a wide array of soluble mediators and stress molecules released and expressed by immune and stromal cells. In further contrast to Th cells, ILCS are continuously present at the peripheral barrier tissues in the body, readily able to produce the first waves of cytokine production upon damage or infection and to strongly shape the ensuing immune response (2). From an evolutionary point of view, the comparable role of ILCs and T cells shows apparent redundancy (3), and while ILC depletion in humans may not be critical for survival (4), ILCs can contribute to the pathogenesis of many diseases (for review, see ref. (5)). 
Conventional NK cells fall within the overarching group of ILCs but are genealogically different from the more recently identified "helper-like" ILCS, which are covered in this review. While the major function of conventional NK cells is inducing cytotoxicity, all other ILCS lack classic cytotoxicity-inducing capacity, and therein lies the distinction between "cytotoxic" and "helper-like" ILCs. Helper-like ILCs are defined by the presence of lymphoid morphology, expression of CD127 (IL-7 receptor a-chain [IL-7Ra]), and lack of lineage markers that are present on other cells (for instance, DCs or T cells). ILCS can be further classified into group 1 (ILC1s), group 2 (ILC2s), and group 3 (ILC3s), which to a great extent mimics the nomenclature that is used for the Th cell groups Th1, Th2, and Th17/Th22, respectively (6). An overview of ILCs and their T cell counterparts is shown in Figure 1.

Innate response

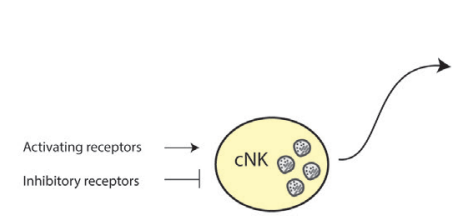

Common effect

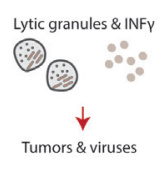

Adaptive response
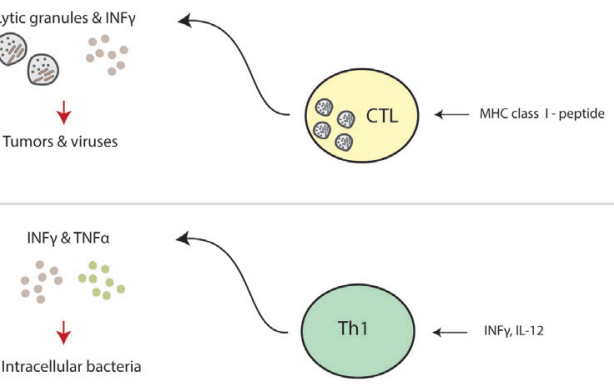

Intracellular bacteria
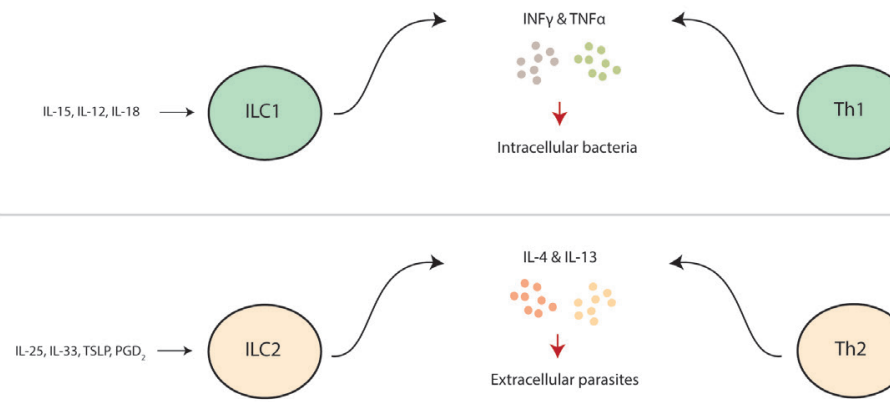

IL-4, IL-25, IL-33, TSLP
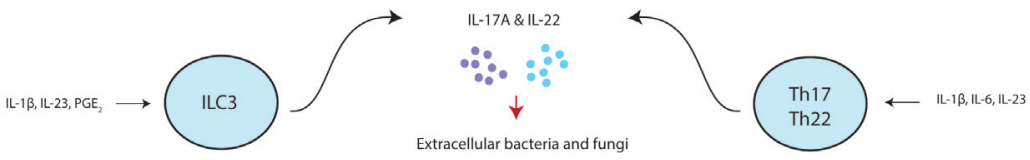

Figure 1

Innate lymphoid cells (ILCS) and their T cell counterparts. Effector functions of ILCs overlap greatly with those of their T cell counterparts. ILCs induce a rapid, innate response (left) that is followed by a slower, adaptive response in T cells (right) targeting the same pathogens. Upstream factors that determine the effector cytokines produced by ILCs and T cells also show considerable overlap. For instance, interleukin-23 (IL-23) is a cytokine involved in the polarization of Th17 cells and is also known to enhance the production of effector cytokines by group 3 ILCS (ILC3s). cNK = conventional natural killer; IFNY = interferon- $\gamma$; CTL = cytotoxic $\mathrm{T}$ lymphocyte; $\mathrm{MHC}=$ major histocompatibility complex; TNF = tumor necrosis factor; TSLP = thymic stromal lymphopoietin; $\mathrm{PGD}_{2}=$ prostaglandin $\mathrm{D}_{2}$. 


\section{ILC development}

Helper-like ILCS are proposed to develop from a "common helper ILC precursor" or CHILP. Various transcription factors are involved in the differentiation of earlier precursors into CHILP. In mice, Id2, NFIL3, and Tox are crucial for the occurrence of all ILCS, while Runx3 is required for the normal development of ILC1s and ILC3s by regulating expression of retinoic acid receptor-related orphan nuclear receptor yt (RORyt) and aryl hydrocarbon receptor (AHR) (7-9). Promyelocytic leukemia zinc-finger protein (PLZF) is a key transcription factor that is required for (most) helper-like ILCs to develop, while PLZF is not needed for conventional NK cell development (10). Studies in mice have also taught us that the transcription factor GATA-3, the prototypical transcription factor of ILC2s, may also be important for the development and function of other ILC groups (11). In mice, GATA-3 is necessary for development of IL-7Ra-expressing ILCS and homeostasis of ILC3s (12). In summary, the balance among the 3 key transcription factors RORyt, GATA3 , and T-bet determines the fate of ILCs in mice $(5,11,13)$. IL-7R signaling not only drives proliferation of ILC3s in mice and humans $(4,14)$, but also stabilizes the ILC3 phenotype in adult mice (15).

The current model of ILC development, depicted in Figure 2, stems from murine data and may not fully mimic the process in humans. A recent review has critically summarized the data on ILC development in humans (16), but a full developmental scheme for ILCs in humans is still lacking. We do know that human CD34+ hematopoietic progenitor cells can, under specific culture conditions, give rise to ILCS (17-19). One group has detected human ILC progenitors in the tonsil and gut lamina propria that developed preferentially into ILC3s when cultured with stem cell factor and AHR signaling, while culture conditions with IL-15 favored the development of conventional NK cells (17). More recently, a similar progenitor population expressing RORyt and Id 2 was identified that in vitro gave rise to all ILC subsets, including conventional NK cells. These ILC progenitors did not develop into other leukocyte lineages and were found exclusively in secondary lymphoid tissues (18). Uncovering the stages of ILC development in humans remains a challenge in the field (16). 


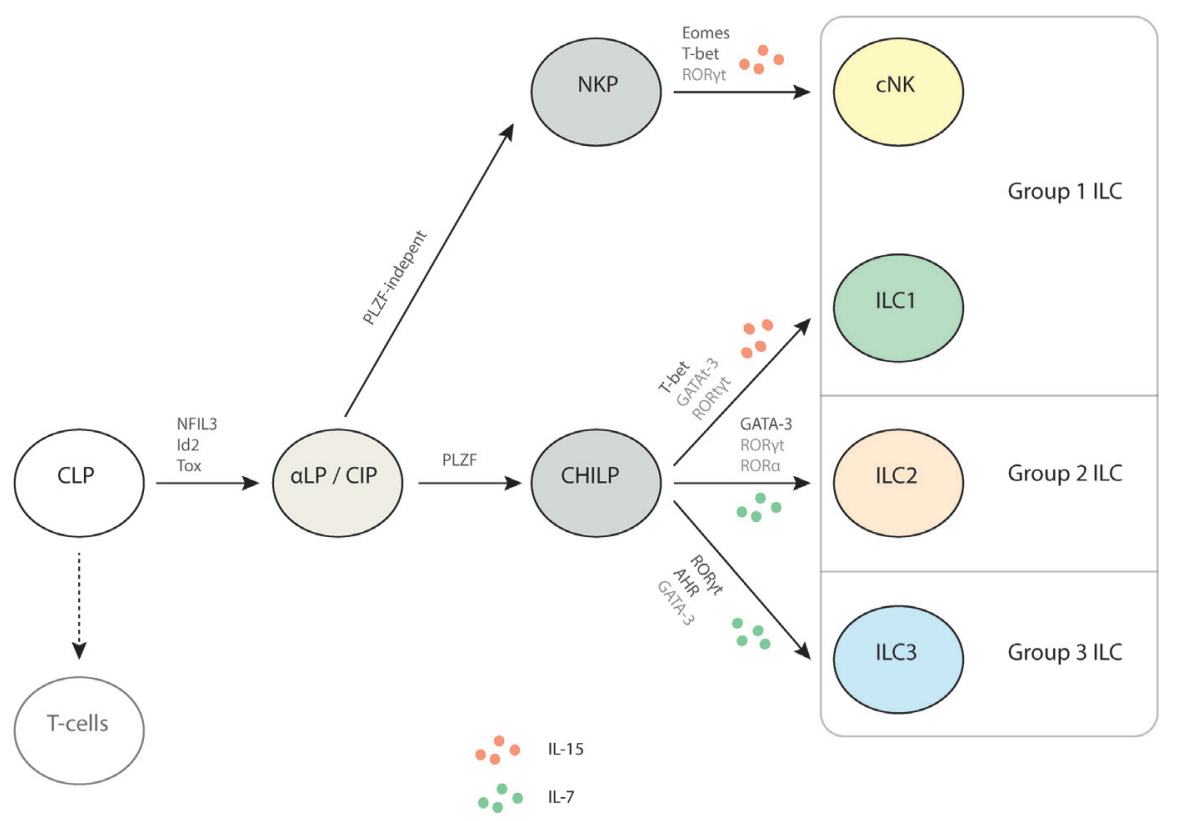

\section{Figure 2}

Development and classification of innate lymphoid cells (ILCS). ILCS develop from the common lymphoid progenitor (CLP), which also gives rise to $T$ cells. The development of the a $4 \beta 7$ integrin-expressing lymphoid progenitor (aLP) is dependent on 3 key transcription factors: NFIL3, Id2, and Tox. After this point, the development splits into natural killer cell precursors (NKP) and a common helper ILC precursor (CHILP), the latter being critically dependent on the transcription factor promyelocytic leukemia zinc finger protein (PLZF). Interleukin-15 (IL-15) is necessary for group 1 ILC (ILC1) development and IL-7 is necessary for ILC2 and ILC3 development. The CHILP gives rise to the 3 helper-like ILCS, each characterized by key transcription factors, namely T-bet, GATA-3, and retinoic acid receptor-related orphan nuclear receptor yt (RORYt) for ILC1s, ILC2s, and ILC3s, respectively. The current model of ILC development, depicted in the figure, stems from murine data. It should be noted that alternate developmental pathways may be possible in humans. For instance, lymphoid tissue inducers, a subset of ILC3s important for lymphoid neogenesis, may not follow the depicted developmental program. In addition, a recent study on ILC progenitors from human secondary lymphoid tissues revealed that a common RORyt-expressing ILC progenitor, probably upstream of the CHILP, was capable of giving rise to all ILCS and that all human ILCs, including conventional natural killer cells (cNK), still express RORyt to some extent (18). Eomes = eomesodermin; AHR = aryl hydrocarbon receptor. 


\section{ILC subsets}

The system of ILC subset classification is based on their developmental pathways, key transcription factors, and function in the form of signature cytokines being produced (6). Briefly, ILC1s express the transcription factor T-bet and produce IFNY; ILC2s express the transcription factor GATA-3 and produce IL-4, IL-5, and IL-13; and ILC3s express the transcription factor RORyt and produce IL-17A and/ or IL-22. Human ILC subsets can be distinguished by the expression of specific cell surface markers (Table 1). Natural cytotoxicity receptors are activating receptors best studied in the context of triggering conventional NK cells, when tumor and virus-infected cells up-regulate their ligands (many of which are still unknown). The natural cytotoxicity receptors include natural killer p30 (NKp30), NKp44 (only expressed in humans), and NKp46, which are also expressed by certain ILCS (20), as will be discussed below.

\begin{tabular}{|c|c|c|c|c|c|}
\hline $\begin{array}{l}\text { Surface } \\
\text { Marker }\end{array}$ & $\begin{array}{l}\text { Conventional } \\
\text { NK cells }\end{array}$ & ILC1s & ILC2s & ILC3s & Interpretation \\
\hline KIR & Yes & no & no & no & Only expressed on conventional NK cells \\
\hline CD94 & yes & no & no & no & $\begin{array}{l}\text { Differentiates between conventional NK } \\
\text { cells and helper-like ILC }\end{array}$ \\
\hline CD56 & yes & some & no & some & $\begin{array}{l}\text { High or moderate expression on } \\
\text { conventional NK cells as well as some } \\
\text { (activated) helper-like ILCs }\end{array}$ \\
\hline $\begin{array}{l}\text { CD127 } \\
\text { (IL-7Ra) }\end{array}$ & no & yes & yes & yes & Expressed on all helper-like ILCs \\
\hline $\begin{array}{l}\text { CD117 } \\
(\mathrm{c}-\mathrm{KIT})\end{array}$ & no & no & some & yes & Expressed by ILC3s and some ILC2s \\
\hline $\begin{array}{l}\text { CD294 } \\
\text { (CRTH) }\end{array}$ & no & no & yes & no & Only expressed on ILC2s \\
\hline NKp44 & yes & some & no & some & $\begin{array}{l}\text { Expressed on conventional NK cells, some } \\
\text { ILC3s and some ILC1s }\end{array}$ \\
\hline
\end{tabular}

Table 1. Cell surface markers used to identify subsets of ILCs in humans

Some of the most common markers used to identify innate lymphoid cells (ILCS) in humans and separate them from conventional natural killer (NK) cells are shown. Within the helper-like ILC group, group 1 ILCS (ILC1s) are defined as CD127+CD117-CD294-; group 2 ILCS are defined as CD127+CD294+; and group 3 ILCS are defined as CD127+CD117+. KIR = killer cell immunoglobulin-like receptor; IL-7Ra = interleukin-7 receptor a-chain; $\mathrm{CRTH} 2$ = chemoattractant receptor-like molecule expressed on Th2 cells; NKp44 = natural killer p44. 


\section{ILC1s}

ILC1s have been proposed to include conventional NK cells and CD127+ and CD103+ ILCS. Although conventional NK cells and ILC1s share many features (e.g., IFNy production), it is becoming clear that these 2 subsets have different progenitors as well as unique tissue distributions and functions (13, 19). ILC1s have been found in intestinal mucosa, salivary gland, liver, and the female reproductive tract; there is a relative paucity of them in blood and lymphoid structures $(21,22)$. On the other hand, conventional NK cells can make up to $15 \%$ of peripheral blood lymphocytes, and substantial numbers of them are present in lymphoid structures. ILC1s are thought to differ from conventional NK cells in that they lack expression of eomesodermin and do not exert cytolytic activity. Overall, ILC1S are a diverse group consisting of subsets that vary depending on their tissue microenvironment and the context of their circumstances (8). For example, small intestine intraepithelial ILC1s have been characterized that did express eomesodermin (23), while mouse ILC1s from salivary glands are capable of TRAILmediated cytotoxicity (24). ILC1s have been suggested to rely on the cytokines IL-15, IL-12, and IL-18, and they produce copious amounts of IFNY and TNF. ILC1S protected mice from infection with Clostridium difficile and Toxoplasma gondii in 2 different infection models, and the ILCs constituted the (early) majority of IFNYproducing cells $(13,25)$. Judging from those studies, the protective role of ILC1S against infections is important. However, exaggerated or insufficient immune responses by ILC1s might lead to immunopathology.

\section{ILC2s}

Consistent with Th2 cells, the transcription factor GATA-3 is the prototypical element required for the development, maintenance, and function of ILC2s. ILC2s are found in increased numbers in the lung, skin, and adipose tissue. They respond to cytokines such as epithelial cell-derived IL-25, IL-33, and thymic stromal lymphopoietin (TSLP) by producing allergy- and fibrosis-related cytokines, such as IL-4, IL-9, and IL-13. Unlike the other ILCs, ILC2s express the prostaglandin $D_{2} \quad\left(P G D_{2}\right)$ receptor chemoattractant receptor-like molecule expressed on Th2 cells (CD294). PGD 2 induces the migration of ILC2s and potentiates their cytokine production (21). For instance, ILC2s express NKp30, which responds to keratinocyte membrane-expressed B7-H6, leading to the production of IL-13, and skin from patients with atopic dermatitis expressed increased levels of B7-H6 (26). ILC2s have diverse functions; they have been shown to promote immunity against extracellular parasites (27), induce tissue repair by producing amphiregulin (28), and promote beige fat and reduce insulin resistance (29). However, deleterious roles of ILC2S have also been described. They are associated with allergies, asthma, and atopic dermatitis. In addition, ILC2s were required for the occurrence of fibrosis in a mouse model of hepatic fibrosis (30). 


\section{ILC3s}

ILC3s are developmentally dependent on expression of the nuclear hormone receptor RORyt $(31,32)$, although in mature ILC3s, this receptor is dispensable for survival and antimicrobial functionality (33). Several subsets of ILC3s have been identified based on function and surface marker expression. The prototypical ILC3s are the lymphoid tissue inducers. These cells are present during fetal development in mice and humans and are responsible for initiation and organization of secondary lymphoid tissues (34-36). Lymphoid tissue inducers interact with stromal and endothelial cells at sites of lymphoid organ development $(37,38)$ and by induction of chemokines and adhesion molecules initiate a cascade of well-described events culminating in the formation of lymph nodes and Peyer's patches (39).

After birth and throughout adulthood, 2 additional ILC3 subsets are present, characterized by the mutually exclusive expression of the natural cytotoxicity receptor NKp46 and the chemokine receptor CCR6 $(40,41)$. Both reside within secondary lymphoid organs as well as in mucosal tissues such as the intestines $(36,42-45)$. Functionally, ILC3s are characterized by the secretion of cytokines associated with Th17 cell immunity, including IL-22, granulocyte-macrophage colony-stimulating factor (GM-CSF), and IL-17A $(36,42-46)$. This cytokine profile and the location of ILC3s at barrier surfaces such as the intestines makes these cells form an integral part of the body's immediate antimicrobial immune response $(40,46-48)$. ILC3s control epithelial production of antimicrobial peptides whose function is to prevent attachment and translocation of intestinal microorganisms (49). In doing so, ILC3s boost the intestines' innate immunity and prevent bacterial dissemination in the wake of maturation of adaptive immunity (50). Taken together, ILC3s are essential for the maintenance of gut homeostasis, for which they use several mechanisms.

Plasticity. It is important to acknowledge the plasticity of ILCs. For example, ILC3s have been demonstrated to differentiate into ILC1-like cells and vice versa under specific inflammatory conditions (51-54). Shifts between other ILCs have also been described (55-57) that help to explain the class, phenotype, and effector function of ILCs detected in diverse tissues under varying environmental circumstances, and plasticity should be taken into account when interpreting the frequency of ILC subsets in rheumatic disease. An overview of the environmental factors that have been shown to steer plasticity between ILC subsets in vitro is shown in Figure 3. 


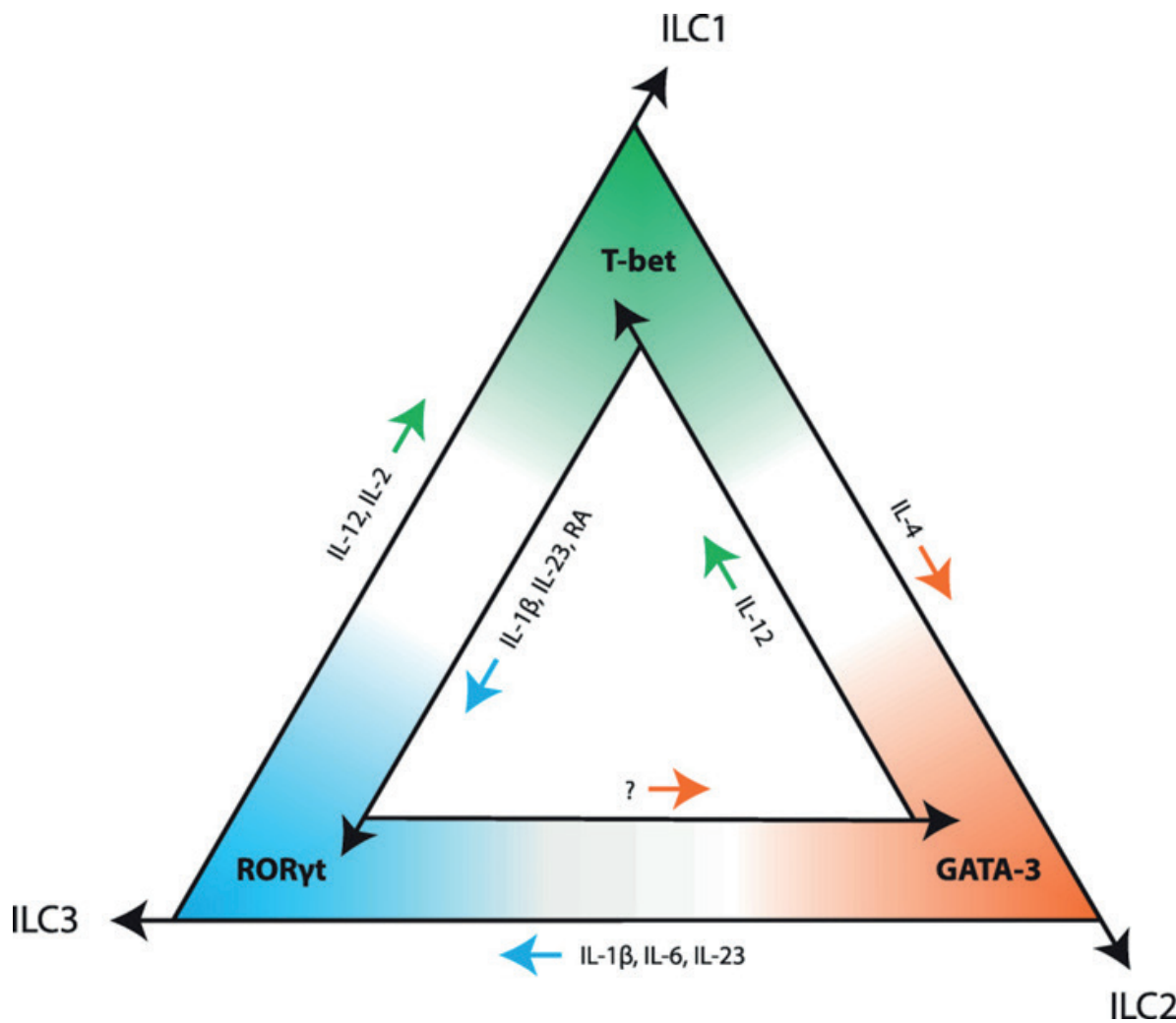

\section{Figure 3}

Plasticity of ILCs. Similar to the known plasticity of Th cells, ILCs can also adapt based on environmental cues, and shifts between ILC groups is a well-established phenomenon. Specific cytokines are depicted that, under culture conditions, have been shown to alter the ILC transcriptional program, cell surface markers, and effector cytokines being produced. Plasticity between ILC1S and ILC3s has been well studied. IL-1 $\beta$, IL-23, and retinoic acid (RA) induce plasticity toward ILC3s, while IL-12 and IL-2 shift the balance toward ILC1S (51-54). The cytokines IL-1 $\beta$, IL-6, and IL-23 have also been shown to induce ILC3-like phenotype and function in ILC2s (56), but it is unclear (?) whether reciprocal plasticity from ILC3s to ILC2s can occur. IL-4 and IL-12 have been shown to balance the plasticity between ILC1s and ILC2s $(55,57)$. See Figure 2 for other definitions. 


\section{Lesson from psoriasis and inflammatory bowel disease (IBD)}

\section{Psoriasis}

IL-22 and IL-17A are key players in the pathogenesis of psoriasis, and it has become evident that conventional $\mathrm{T}$ cells are not the only contributors to the production of these effector cytokines (58). Although the NKp44+ ILC3 population is very scarce in the peripheral blood, these cells represent a major proportion of the ILC population in healthy skin (58-60), and numerous independent studies have shown that NKp44+ ILC3s are even more abundant in skin and blood samples from patients with psoriasis (58-62).

One very interesting finding pops up from studies of ILCs in psoriasis. The frequency of specifically NKp44+ ILC3s in the unaffected skin of psoriasis patients is equal to if not higher than the frequency of NKp44+ ILC3s in affected skin $(58,59)$. This altered composition of ILCs in what would clinically appear to be unaffected skin of psoriasis patients raises the key question: are these NKp44+ ILC3s pathogenic or protective? The most common interpretation is that NKp44+ ILC3s in unaffected skin are prepositioned to initiate an inflammatory cascade $(58-60,62)$. Animal models show that IL-22 causes skin lesions reminiscent of psoriasis, which supports the notion that NKp44+ ILC3s are necessary and critical for this process $(63,64)$. Under culture conditions, the cytokines IL-1 $\beta$ and IL-23 can shift ILC1 and NKp44- ILC3 populations toward NKp44+ ILC3s $(51,62)$. Recent gene expression profiling shows convincingly that "unaffected" psoriatic skin is quite different from skin of healthy individuals, with "already" significantly increased transcripts of IL$1 \beta, \mathrm{IL}-22$, and IL-17 (65). Taken together with the established genetic background of psoriasis patients that includes single-nucleotide polymorphisms (SNPs) in IL23R (66), these findings might explain why ILC composition in unaffected skin of psoriasis patients is altered.

Our knowledge of ILC3 function mainly stems from mouse models or, in humans, from tonsil- or gut-derived ILCs. It is known that NKp44+ ILC3s from psoriatic skin lesions are capable of producing IL-22 to a much greater extent than IL-17A (62), but it would be an oversimplification to view IL-22 as purely pathogenic, since this cytokine has essential regulatory and regenerative functions (for review, see ref. (67)) highlighted below. 


\section{IBD}

ILCS have important functions in homeostasis of the intestinal tract and in establishing immune equilibrium with commensal bacteria (41). It is therefore not surprising that the loss of intestinal homeostasis and subsequent pathologic antimicrobial immunity that drive IBD are associated with alterations in intestinal ILCs (for review, see ref. (68)).

In stark contrast to healthy skin, under homeostatic conditions the healthy gut is characterized by an abundant, protective NKp44+ ILC3 population, which reciprocally shifts toward a predominance of ILC1S in IBD, meaning a significantly increased proportion of ILCs that secrete IFNy $(23,51,52,69)$. Additionally, ILC3s from healthy gut mainly produce the homeostatic cytokine IL-22, while ILC3s in IBD also secrete proinflammatory IL-17A (70). Animal models have also taught us that ILC3s can dampen pathogenic CD4+ T cell responses (48) and that ILC3-derived IL-22 protects against colitis (71) and induces tolerance to commensal bacteria $(47,72)$. The differential phenotype and function of ILCS in psoriasis versus IBD emphasizes that the outcome of ILC function (pathogenic versus protective) is highly dependent on the environment and tissue in which these cells are located.

\section{Studies of ILCs in rheumatic disease}

\section{Psoriatic arthritis (PsA)}

ILCS have been found in increased numbers in synovial fluid from PsA patients (61). Synovial fluid from PsA patients and patients with rheumatoid arthritis (RA) showed an increase in frequency of ILC1s as compared to the frequency of ILC1S in peripheral blood, while the frequency of ILC2s was unaltered. Interestingly, only synovial fluid from PsA patients contained a major increase in NKp44+ ILC3s, which distinguished the synovial compartment in PsA from that in RA. The increased presence of synovial fluid ILC3s also coincided with up-regulated expression of CCR6. In contrast, peripheral blood from PsA patients contained fewer CCR6+ ILCs than did peripheral blood from healthy controls, and the frequency of CCR6+ ILC3s was inversely related to disease activity measures, which could indicate specific targeting of these ILC3s to the joint in patients with PSA. ILC3s in synovial fluid from PsA patients were capable of IL-17A production, implicating their role in PsA pathogenesis (61).

PsA has recently been thought of as a disease that starts at the enthesis with microtrauma and culminates in chronic inflammation. In contrast to mouse models of psoriasis and colitis, the role of ILCs has not been studied in experimental models of PSA. It is of particular interest to determine the relative importance of ILC3s and 
other innate lymphocytes, such as IL-17A-producing $ү / \delta T$ cells, as the latter have been demonstrated to be present in mouse models of enthesitis $(73,74)$. Only one group has described the presence of ILC3s and ILC2s in nonrheumatic human spinal, anterior cruciate ligament, and Achilles tendon entheses, yet these data need to be published to be fully appreciated (75). Future work should evaluate which activating/inhibitory receptors are expressed by ILCS found in the joint and determine how their ligands expressed by tissue resident cells influence disease pathogenesis (76-79), for instance, by affecting production of proinflammatory cytokines by ILCS.

\section{Ankylosing spondylitis (AS)}

AS is characterized by inflammatory back pain and axial new bone formation. Our current view of AS is that the cytokines TNF, IL-17, and IL-22 are critical drivers of its pathogenesis $(73,80,81)$. Animal models have further shown that innate counterparts to $T$ cells can produce these cytokines, mimicking the clinical manifestations of AS $(73,74,81)$. ILC3s have been described in nonrheumatic enthesis (75), and there is evidence of the increased presence of IL-22-producing NKp44+ ILC3s in other tissues from AS patients. Ciccia et al described a cell subset consisting of CD56+NKp44+ cells, possibly ILC3s, to be the main producers of IL22 in the gut of AS patients. In contrast, in Crohn's disease, CD4+ T cells were the main producers of IL-22 (82). In a follow-up study, Ciccia et al found an increased presence of a4 7 integrin-positive IL-17+ and IL-22+ ILC3s in the gut, blood, synovial fluid, and bone marrow (83). The interesting hypothesis was put forward that gut-derived ILC3s might home to inflamed (sacroiliac) joints and contribute to the disease process by producing IL-17 and IL-22 (83). A role for gut-derived immune cells is underscored by the transgenic HLA-B27 rat model of AS, in which the gut microbiome is necessary for the occurrence of disease $(84,85)$. Consistent with PSA, a genetic background could enhance the role of ILC3s in the pathogenesis of AS. IL-23R, RUNX3, and MICA have all been linked to AS susceptibility (86).

A recent study further demonstrated that $P G E_{2}$, via the $\mathrm{EP}_{4}$ receptor, potentiates gut ILC3 proliferation and IL-22 production (87). The effect of nonsteroidal antiinflammatory drugs on ILC3 proliferation and IL-22 production deserves investigation in the context of AS. 


\section{RA}

RA is a disease driven by MHC class II molecules and adaptive immune responses, exemplified by the effectiveness of drugs targeting $B$ cells and the interaction between costimulatory molecules and CD28 (CTLA-4Ig). ILCs have been implicated in supporting antibody production by $B$ cells via diverse pathways, including lymphotoxin, CD40, and BAFF/APRIL signaling (88). Adult ILC3s can sustain CD4+ $T$ cell memory-enhancing germinal center reactions and subsequent antibody production (89). As discussed in the section on ILC3s, these cells are important in the development and homeostasis of secondary lymphoid tissue (82). Under inflammatory conditions, so-called tertiary lymphoid structures can develop, which are germinal center-like structures that arise after birth at sites not originally in need of lymphoid structures.

This prompted a study to investigate if ILCS could be found in tertiary lymphoid structures of RA synovial tissue, but hardly any ILC3s were detected (90). Another research group set out to characterize ILCs in inguinal lymph nodes in patients with preclinical RA (arthralgia patients positive for rheumatoid factor and/or anticitrullinated protein antibodies), patients with established RA, and healthy controls (91). Although ILCs were sparsely present and there were no differences in total ILC numbers, it was found that in the lymph nodes of patients with preclinical RA, the ILC1/2 group (the study was hampered by not distinguishing between ILC1s and ILC2s) made up a larger proportion of the ILCs than in the lymph nodes of healthy controls. Interestingly, there was a shift in the ILC3 population from NKp44- ILC3s to NKp44+ ILC3s in patients with established RA and a clear tendency toward fewer NKp44- ILC3s in patients with preclinical RA. Since NKp44- ILC3s are thought to be involved in maintaining secondary lymphoid tissue homeostasis (lymphoid tissue inducers), it was hypothesized that the loss of these cells may result in an autoimmune disease-prone lymph node microenvironment (91).

The above-described paucity of ILC3s in RA synovial tertiary lymphoid structures (90) is consistent with the relative lack of ILC3s described in RA synovial fluid (61) and makes a role for ILC3s in the local RA disease process less likely. In contrast to ILC3s and ILC2s, ILC1s can readily be found in the RA synovial cavity, and the frequency of ILC1s there is strongly increased compared to the frequency of ILC1s in peripheral blood (61). It appears that if ILCs play a role in local RA pathology, then ILC1s are the subset to look at and could contribute to the production of TNF and IFNY. 


\section{Sjögren's syndrome (SS)}

ILCs have been identified in the salivary glands of mice $(22,24,92)$ and humans (93). The established role of ILCs in producing IL-22 and IL-17A, which are involved in the pathogenesis of primary SS (94), along with their presence at epithelial sites of the body poses ILCS as candidate contributors to the pathogenesis of primary SS. Specifically, it was shown that IL-22-producing NKp44+ ILC3s were more abundant in the salivary glands of patients with primary SS than in the salivary glands of patients with sicca syndrome without SS, and that the frequency of the ILC3s was positively correlated with the lymphocytic focus score (93). Additional studies will be needed to confirm these results and to further evaluate the function of ILC3s in salivary glands of patients with primary SS.

Three key areas deserve subsequent exploration in the setting of primary SS. First, ILCs from mice appear to be "tissue resident" in the salivary gland, meaning that they are barely replenished from circulating (precursor) ILCs that would subsequently migrate into the salivary gland $(22,92)$. Second, the histopathology of SS is marked by the formation of tertiary lymphoid structures in salivary glands, which suggests the involvement of ILCs $(36,95)$. Finally, SNPs in NKp30 that cause decreased expression of this receptor are less common in patients with primary SS, and, indeed, patients with primary SS have increased expression of NKp30 on conventional NK cells (96). Helper-like ILCs can also express this receptor, and it is known that the ligand for NKp30, namely B7-H6, is expressed by salivary gland epithelial cells (96). Taken together, these observations highlight the potential role of ILCs in primary SS and justify future research.

\section{Systemic lupus erythematosus (SLE)}

ILCS have been described in patients with SLE as part of a control cohort for a study on systemic sclerosis (SSc), and the frequency of ILC2s was found to be increased in their circulation compared to that in healthy controls (97). The noted elevated frequency of ILC2s is difficult to reconcile with the established effect of IFNs of decreasing ILC survival and function. SLE is clearly marked by an IFN signature (98), yet studies in mice have shown that ILC3s undergo CD95/FasL-mediated apoptosis following the production of type I IFNs by plasmacytoid DCs (99). Similarly, type I IFNs also increase cell death and decrease cytokine production by ILC2s (100).

Patients with SLE are prone to develop skin lesions (e.g., butterfly rash) following sunlight exposure (ultraviolet B) that induces excessive keratinocyte apoptosis (101). ILCs are important in skin homeostasis, and this function is mediated by production of IL-22, a key cytokine that helps prevent keratinocyte damage (102, 103). In this context, the (protective) role of IL-22-producing ILCS in epithelial homeostasis is an interesting avenue for investigation that could shed light on some of the cutaneous manifestations of the disease (104). 


\section{SSc}

SSc is marked by low-grade inflammation, vascular injury, and subsequent fibrosis of skin and internal organs. In a recent study, ILC2s were present in markedly increased numbers in the skin and blood of SSc patients. Numbers of ILC2s were elevated the most in patients with diffuse cutaneous SSC and correlated with the modified Rodnan skin thickness score $(97,105)$. In light of the IFN signature (which is strongest in early and nonfibrotic SSc (106)) and the suppressive effect of IFN on ILC2s (100), the increased numbers of ILC2s in more advanced stages of the disease is noteworthy (97). The increased presence of ILC2s in SSc was not found in another study that compared 38 clinically undefined SSc patients with matched healthy controls (107). It is unclear why there was such a difference in outcome between the 2 studies, but different methodologies (e.g., different cell markers and gating strategy used) could help explain the discrepancy.

With regard to the occurrence of fibrosis in SSc, the ILC2 subset is of particular interest. In mice, ILC2s were required for the occurrence of hepatic fibrosis, and IL-13 released by ILC2s was sufficient for collagen deposition in a mouse model of pulmonary fibrosis $(30,108)$. ILC2s are activated, start producing cytokines (e.g., IL-13), and proliferate upon exposure to epithelial alarmin cytokines, such as IL-25, IL-33, and TSLP, all of which are found to be elevated in SSC (109). ILC2-derived IL13 induces collagen deposition from fibroblasts and differentiates macrophages toward a profibrotic phenotype (108).

NKp44+ ILC3s are an important source of IL-17A in skin inflammation, and IL-17A has been implicated in the generation of pulmonary and skin fibrosis (110). In patients with SSc, the NKp44+ ILC3 population was slightly increased in blood, while the NKp44- ILC3 population was decreased compared to that in healthy controls (107). In addition, the same study found an increase in CD4+ ILC1s, which were potent producers of TNF and GM-CSF, in the peripheral blood of SSc patients (107). Future studies should examine the presence and function of ILCS at affected sites, such as the skin, to better understand their potential contribution to SSC. 


\section{Conclusions}

Over the past few years, it has become clear that ILCs play a pivotal role in orchestrating the first waves of the immune response in human tissue upon receiving danger signals, thereby contributing toward effective clearance of the initiating event. On the other hand, numerous studies have shown the relevance of ILCs in upholding tissue homeostasis. Clear evidence of this balancing act has been well studied in skin and gut disease, yet an overview of the contribution of ILCS to rheumatic disease has been lacking. In this review, we provide a comprehensive overview of the recent research that has been performed on helper-like ILCs in rheumatic diseases (Table 2). Recent reports have begun to shed light on ILC aberrations in patients with IBD-associated arthritis (111) and antineutrophil cytoplasmic antibody-associated vasculitis (112). Many other rheumatic diseases, such as Behçet's disease and reactive arthritis, await and deserve further exploration into the role of ILCS, and more work is needed on the diseases highlighted in this review.

We believe that future work should focus on 3 key issues. At first, due to the rarity of ILCs, our knowledge of these cells is largely based on flow cytometric phenotyping. Hence, we encourage the ILC field to adhere to the established framework for ILC gating to improve comparability between studies and between different clinical conditions as well as to use standardized measures before and after initiation of therapeutic intervention. Second, the low frequency of ILCs challenges our ability to isolate ILCS from human tissues for the purpose of functional assays. For instance, the culturing of ILCs to focus on their functional roles in health and disease poses challenges that hinder development of drugs to target pathologic processes that they orchestrate. Until now, our current knowledge of ILC development and function has been mainly based on experimental models, which leaves its relevance for humans to be determined. The use of alternative, novel techniques that give an understanding of human ILC function and development (e.g., single-cell sequencing $(113,114)$ ) should therefore be exploited further.

Finally, strategies aimed at targeting ILC pathology by blocking their developmental fate, plasticity, and/or effector function deserve further investigation (115). Do therapies targeting the IL-23/IL-17 axis given to patients with psoriatic skin disease also halt aberrant ILC function in the joint or enthesis, preventing the transition toward concomitant joint disease? If this hypothesis holds true, we may go on to apply similar ways of delivering preventative medicine to patients with other rheumatic diseases, shifting our focus away from targeting the chronic effects of immune-mediated disease and toward disease interception, possibly by tackling the ILCS. 


\begin{tabular}{|c|c|c|}
\hline Disease & Implicated ILCs & Role in pathogenesis (ref.) \\
\hline \multicolumn{3}{|l|}{ Psoriasis } \\
\hline & NKp44+ ILC3s & $\begin{array}{l}\text { Increased frequency in blood and in unaffected and } \\
\text { affected skin (58-62) }\end{array}$ \\
\hline \multicolumn{3}{|l|}{ IBD } \\
\hline & ILC3s & Increased IL-17A production by ILC3s (70) \\
\hline & ILC1s & $\begin{array}{l}\text { Overall shift from ILC3s toward IFNy-producing ILC1s ( } 23 \text {, } \\
\text { 52) }\end{array}$ \\
\hline \multicolumn{3}{|l|}{ PsA } \\
\hline & ILC3s and ILC2s & Present in nonrheumatic enthesis (75) \\
\hline & CCR6+ ILC3S & $\begin{array}{l}\text { Shift from blood to synovial cavity correlates with disease } \\
\text { severity (61) }\end{array}$ \\
\hline & NKp44+ ILC3s & $\begin{array}{l}\text { Highly increased presence in synovial joint in PsA patients } \\
\text { in comparison to blood and in comparison to RA patients } \\
\text { (61) }\end{array}$ \\
\hline \multicolumn{3}{|c|}{ IBD-associated } \\
\hline arthritis & $\begin{array}{l}\text { IFNy+ ILCs and IL-17A+ } \\
\text { ILCs }\end{array}$ & $\begin{array}{l}\text { Increased frequency in blood compared to IBD patients } \\
\text { without arthritis (111) }\end{array}$ \\
\hline \multicolumn{3}{|l|}{ AS } \\
\hline & ILC3s and ILC2s & Present in nonrheumatic enthesis (75) \\
\hline & $\begin{array}{l}\text { a4 } \beta 7 \text { integrin-positive IL- } \\
17+\text { and IL-22+ ILC3s }\end{array}$ & $\begin{array}{l}\text { Increased presence in gut, blood, bone marrow, and } \\
\text { joints in AS (83) }\end{array}$ \\
\hline \multicolumn{3}{|l|}{ RA } \\
\hline & ILC1s & Selectively increased in synovial fluid of RA patients (61) \\
\hline & NCR-ILC3S & Reduced numbers in lymph nodes in (preclinical) RA (91) \\
\hline \multicolumn{3}{|l|}{ SS } \\
\hline & NKp44+ ILC3s & $\begin{array}{l}\text { Increased in salivary gland and correlate with lymphocytic } \\
\text { focus score (93) }\end{array}$ \\
\hline \multicolumn{3}{|l|}{ SLE } \\
\hline & ILC2s & Elevated frequency in blood (97) \\
\hline \multicolumn{3}{|c|}{ ANCA-associated } \\
\hline vasculitis & ILC2s and ILC3s & $\begin{array}{l}\text { Decreased frequency in blood of patients with acute } \\
\text { disease (112) }\end{array}$ \\
\hline \multicolumn{3}{|l|}{ SSc } \\
\hline & ILC2s & $\begin{array}{l}\text { Increased in skin and blood of SSc patients, and numbers } \\
\text { correlated with skin fibrosis and ILD (97); no increased } \\
\text { numbers found in another study (107) }\end{array}$ \\
\hline & $\begin{array}{l}\text { NKp44+ ILC3s and } \\
\text { NKp44- ILC3s }\end{array}$ & $\begin{array}{l}\text { Found increased and decreased, respectively, in blood } \\
\text { (107) }\end{array}$ \\
\hline & CD4+ ILC1S & Increased in blood of SSc patients (107) \\
\hline
\end{tabular}

Table 2. Studies implicating ILCs in rheumatic and related epithelial diseases in humans.

ILCS = innate lymphoid cells; NKp44 = natural killer p44; ILC3s = group 3 ILCs; IBD = inflammatory bowel disease; IL-17A = interleukin-17A; IFNy = interferon-ү; PsA = psoriatic arthritis; RA = rheumatoid arthritis; AS = ankylosing spondylitis; NCR = natural cytotoxicity receptor; SS = Sjögren's syndrome; SLE = systemic lupus erythematosus; ANCA = antineutrophil cytoplasmic antibody; SSC = systemic sclerosis; ILD = interstitial lung disease. 


\section{References}

1. Exley MA, Tsokos GC, Mills KH, Elewaut D, Mulhearn B. What rheumatologists need to know about innate lymphocytes. Nat Rev Rheumatol 2016;12:658-68.

2. Bando JK, Colonna M. Innate lymphoid cell function in the context of adaptive immunity. Nat Immunol 2016;17:783-9.

3. Vivier E, van de Pavert SA, Cooper MD, Belz GT. The evolution of innate lymphoid cells. Nat Immunol 2016;17:790-4.

4. Vély F, Barlogis V, Vallentin B, Neven B, Piperoglou C, Ebbo M, et al. Evidence of innate lymphoid cell redundancy in humans. Nat Immunol 2016;17:1291-9.

5. Klose CS, Artis D. Innate lymphoid cells as regulators of immunity, inflammation and tissue homeostasis. Nat Immunol 2016; 17:765-74.

6. Spits H, Artis D, Colonna M, Diefenbach A, Di Santo JP, Eberl G, et al. Innate lymphoid cells: a proposal for uniform nomenclature. Nat Rev Immunol 2013;13:145-9.

7. Gronke K, Nielsen MK, Diefenbach A. Innate lymphoid cells, precursors and plasticity. Immunol Lett 2016;179:9-18.

8. Fuchs A. ILC1s in tissue inflammation and infection. Front Immunol 2016;7:104.

9. Ebihara T, Song C, Ryu SH, Plougastel-Douglas B, Yang L, Levanon D, et al. Runx3 specifies lineage commitment of innate lymphoid cells. Nat Immunol 2015;16:1124-33.

10. Constantinides MG, McDonald BD, Verhoef PA, Bendelac A. A committed precursor to innate lymphoid cells. Nature 2014; 508:397-401.

11. Zhong C, Cui K, Wilhelm C, Hu G, Mao K, Belkaid Y, et al. Group 3 innate lymphoid cells continuously require the transcription factor GATA-3 after commitment. Nat Immunol 2016; 17:169-78.

12. Yagi R, Zhong C, Northrup DL, Yu F, Bouladoux N, Spencer S, et al. The transcription factor GATA3 is critical for the development of all IL-7Ra-expressing innate lymphoid cells. Immunity 2014;40:378-88.

13. Klose CS, Flach M, M€ohle L, Rogell L, Hoyler T, Ebert K, et al. Differentiation of type 1 ILCS from a common progenitor to all helper-like innate lymphoid cell lineages. Cell 2014;157: 340-56.

14. Schmutz S, Bosco N, Chappaz S, Boyman O, Acha-Orbea H, Ceredig R, et al. Cutting edge: IL-7 regulates the peripheral pool of adult RORg+ lymphoid tissue inducer cells. J Immunol 2009;183:2217-21.

15. Vonarbourg C, Mortha A, Bui VL, Hernandez PP, Kiss EA, Hoyler T, et al. Regulated expression of nuclear receptor RORgt confers distinct functional fates to NK cell receptor expressing RORgt+ innate lymphocytes. Immunity 2010;33:736-51.

16. Juelke K, Romagnani C. Differentiation of human innate lymphoid cells (ILCs). Curr Opin Immunol 2016;38:75-85.

17. Montaldo E, Teixeira-Alves LG, Glatzer T, Durek P, Stervbo U, Hamann W, et al. Human RORgt+CD34+ cells are lineage specified progenitors of group 3 RORgt+ innate lymphoid cells. Immunity 2014;41:988-1000. 
18. Scoville SD, Mundy-Bosse BL, Zhang MH, Chen L, Zhang X, Keller KA, et al. A progenitor cell expressing transcription factor RORgt generates all human innate lymphoid cell subsets. Immunity 2016;44:1140-50.

19. Renoux VM, Zriwil A, Peitzsch C, Micha€elsson J, Friberg D, Soneji S, et al. Identification of a human natural killer cell lineage-restricted progenitor in fetal and adult tissues. Immunity 2015;43:394-407.

20. Kruse PH, Matta J, Ugolini S, Vivier E. Natural cytotoxicity receptors and their ligands. Immunol Cell Biol 2014;92:221-9.

21. Montaldo E, Vacca P, Vitale C, Moretta F, Locatelli F, Mingari MC, et al. Human innate lymphoid cells. Immunol Lett 2016; 179:2-8.

22. Gasteiger G, Fan X, Dikiy S, Lee SY, Rudensky AY. Tissue residency of innate lymphoid cells in lymphoid and nonlymphoid organs. Science 2015;350:981-5.

23. Fuchs A, Vermi W, Lee JS, Lonardi S, Gilfillan S, Newberry RD, et al. Intraepithelial type 1 innate lymphoid cells are a unique subset of IL-12- and IL-15-responsive IFN-g-producing cells. Immunity 2013;38:769-81.

24. Schuster IS, Wikstrom ME, Brizard G, Coudert JD, Estcourt MJ, Manzur M, et al. TRAIL+ NK cells control CD4+ $\mathrm{T}$ cell responses during chronic viral infection to limit autoimmunity. Immunity 2014;41:646-56.

25. Abt MC, Lewis BB, Caballero S, Xiong H, Carter RA, Susac B, et al. Innate immune defenses mediated by two ILC subsets are critical for protection against acute Clostridium difficile infection. Cell Host Microbe 2015;18:27-37.

26. Salimi M, Xue L, Jolin H, Hardman C, Cousins DJ, McKenzie AN, et al. Group 2 innate lymphoid cells express functional NKp30 receptor inducing type 2 cytokine production. J Immunol 2016;196:45-54.

27. Neill DR, Wong SH, Bellosi A, Flynn RJ, Daly M, Langford TK, et al. Nuocytes represent a new innate effector leukocyte that mediates type-2 immunity. Nature 2010;464:1367-70.

28. Turner JE, Morrison PJ, Wilhelm C, Wilson M, Ahlfors H, Renauld JC, et al. IL-9-mediated survival of type 2 innate lymphoid cells promotes damage control in helminth-induced lunginflammation. J Exp Med 2013;210:2951-65.

29. McKenzie AN, Spits H, Eberl G. Innate lymphoid cells in inflammation and immunity. Immunity 2014;41:366-74.

30. McHedlidze T, Waldner M, Zopf S, Walker J, Rankin AL, Schuchmann M, et al. Interleukin-33dependent innate lymphoid cells mediate hepatic fibrosis. Immunity 2013;39:357-71.

31. Eberl G, Marmon S, Sunshine MJ, Rennert PD, Choi Y, Littman DR. An essential function for the nuclear receptor RORgt in the generation of fetal lymphoid tissue inducer cells. Nat Immunol 2004;5:64-73.

32. Sun Z, Unutmaz D, Zou YR, Sunshine MJ, Pierani A, Brenner- Morton S, et al. Requirement for RORg in thymocyte survival and lymphoid organ development. Science 2000;288:2369-73.

33. Withers DR, Hepworth MR, Wang X, Mackley EC, Halford EE, Dutton EE, et al. Transient inhibition of ROR-gt therapeutically limits intestinal inflammation by reducing TH17 cells and preserving group 3 innate lymphoid cells. Nat Med 2016;22:319-23. 


\section{CHAPTER 5}

34. Mebius RE, Rennert P, Weissman IL. Developing lymph nodes collect CD4+CD3- LTb+ cells that can differentiate to APC, NK cells, and follicular cells but not T or B cells. Immunity 1997;7:493-504.

35. Van de Pavert SA, Mebius RE. New insights into the development of lymphoid tissues. Nat Rev Immunol 2010;10:664-74.

36. Cupedo T, Crellin NK, Papazian N, Rombouts EJ, Weijer K, Grogan JL, et al. Human fetal lymphoid tissue-inducer cells are interleukin 17-producing precursors to RORC+CD127+ natural killer-like cells. Nat Immunol 2009;10:66-74.

37. Onder L, Danuser R, Scandella E, Firner S, Chai Q, Hehlgans T, et al. Endothelial cell-specific lymphotoxin-b receptor signaling is critical for lymph node and high endothelial venule formation. J Exp Med 2013;210:465-73.

38. Yoshida H, Naito A, Inoue JI, Satoh M, Santee-Cooper SM, Ware CF, et al. Different cytokines induce surface lymphotoxin-ab on IL-7 receptor-a cells that differentially engender lymph nodes and Peyer's patches. Immunity 2002;17:823-33.

39. Randall TD, Carragher DM, Rangel-Moreno J. Development of secondary lymphoid organs. Annu Rev Immunol 2008;26:627-50.

40. Spits $\mathrm{H}$, Cupedo $\mathrm{T}$. Innate lymphoid cells: emerging insights in development, lineage relationships, and function. Annu Rev Immunol 2012;30:647-75.

41. Artis D, Spits H. The biology of innate lymphoid cells. Nature 2015;517:293-301.

42. Luci C, Reynders A, Ivanov II, Cognet C, Chiche L, Chasson L, et al. Influence of the transcription factor RORgt on the development of NKp46+ cell populations in gut and skin. Nat Immunol 2009;10:75-82.

43. Sanos SL, Bui VL, Mortha A, Oberle K, Heners C, Johner C, et al. RORgt and commensal microflora are required for the differentiation of mucosal interleukin 22-producing NKp46+ cells. Nat Immunol 2009;10:83-91.

44. Satoh-Takayama N, Vosshenrich CA, Lesjean-Pottier S, Sawa S, Lochner M, Rattis F, et al. Microbial flora drives interleukin 22 production in intestinal NKp46+ cells that provide innate mucosal immune defense. Immunity 2008;29:958-70.

45. Cella M, Fuchs A, Vermi W, Facchetti F, Otero K, Lennerz JK, et al. A human natural killer cell subset provides an innate source of IL-22 for mucosal immunity. Nature 2009;457:722-5.

46. Spits H, Di Santo JP. The expanding family of innate lymphoid cells: regulators and effectors of immunity and tissue remodeling. Nat Immunol 2011;12:21-7.

47. Goto Y, Obata T, Kunisawa J, Sato S, Ivanov II, Lamichhane A, et al. Innate lymphoid cells regulate intestinal epithelial cell glycosylation. Science 2014;345:1254009.

48. Hepworth MR, Monticelli LA, Fung TC, Ziegler CG, Grunberg S, Sinha R, et al. Innate lymphoid cells regulate CD4+ T-cell responses to intestinal commensal bacteria. Nature 2013;498:113-7.

49. Sonnenberg GF, Fouser LA, Artis D. Border patrol: regulation of immunity, inflammation and tissue homeostasis at barrier surfaces by IL-22. Nat Immunol 2011;12:383-90.

50. Sonnenberg GF, Monticelli LA, Elloso MM, Fouser LA, Artis D. CD4+ lymphoid tissue-inducer cells promote innate immunity in the gut. Immunity 2011;34:122-34. 
51. Bernink JH, Krabbendam L, Germar K, de Jong E, Gronke K, Kofoed-Nielsen M, et al. Interleukin-12 and -23 control plasticity of $\mathrm{Cd} 127+$ group 1 and group 3 innate lymphoid cells in the intestinal lamina propria. Immunity 2015;43:146-60.

52. Bernink JH, Peters CP, Munneke $M$, te Velde AA, Meijer SL, Weijer K, et al. Human type 1 innate lymphoid cells accumulate in inflamed mucosal tissues. Nat Immunol 2013;14:221-9.

53. Klose CS, Kiss EA, Schwierzeck V, Ebert K, Hoyler T, D'Hargues Y, et al. A T-bet gradient controls the fate and function of CCR62RORgt+ innate lymphoid cells. Nature 2013;494: 261-5.

54. Cella M, Otero K, Colonna M. Expansion of human NK-22 cells with IL-7, IL-2, and IL-1b reveals intrinsic functional plasticity. Proc Natl Acad Sci U S A 2010;107:10961-6.

55. Lim Al, Menegatti S, Bustamante J, Le Bourhis L, Allez M, Rogge L, et al. IL-12 drives functional plasticity of human group 2 innate lymphoid cells. J Exp Med 2016;213:569-83.

56. Huang Y, Guo L, Qiu J, Chen X, Hu-Li J, Siebenlist U, et al. IL-25-responsive, lineage-negative KLRG1 hi cells are multipotential "inflammatory" type 2 innate lymphoid cells. Nat Immunol 2015;16:161-9.

57. Bal SM, Bernink JH, Nagasawa M, Groot J, Shikhagaie MM, Golebski K, et al. IL-1b, IL-4 and IL-12 control the fate of group 2 innate lymphoid cells in human airway inflammation in the lungs. Nat Immunol 2016;17:636-45.

58. Villanova F, Flutter B, Tosi I, Grys K, Sreeneebus H, Perera GK, et al. Characterization of innate lymphoid cells in human skin and blood demonstrates increase of NKp44+ ILC3 in psoriasis. J Invest Dermatol 2014;134:984-91.

59. Dyring-Andersen B, Geisler C, Agerbeck C, Lauritsen JP, G_udjonsdottir SD, Skov L, et al. Increased number and frequency of group 3 innate lymphoid cells in nonlesional psoriatic skin. Br J Dermatol 2014;170:609-16.

60. Bruggen MC, Bauer W, Reininger B, Clim E, Captarencu C, Steiner GE, et al. In situ mapping of innate lymphoid cells in human skin: evidence for remarkable differences between normal and inflamed skin. J Invest Dermatol 2016;136:2396-405.

61. Leijten EF, van Kempen TS, Boes M, Michels-van Amelsfort JM, Hijnen D, Hartgring SA, et al. Enrichment of activated group 3 innate lymphoid cells in psoriatic arthritis synovial fluid. Arthritis Rheumatol 2015;67:2673-8.

62. Teunissen MB, Munneke JM, Bernink JH, Spuls PI, Res PC, te Velde A, et al. Composition of innate lymphoid cell subsets in the human skin: enrichment of NCR+ ILC3 in lesional skin and blood of psoriasis patients. J Invest Dermatol 2014;134:2351-60.

63. Pantelyushin S, Haak S, Ingold B, Kulig P, Heppner FL, Navarini AA, et al. Rorgt1 innate lymphocytes and gd T cells initiate psoriasiform plaque formation in mice. J Clin Invest 2012;122:2252-6.

64. Ma HL, Liang S, Li J, Napierata L, Brown T, Benoit S, et al. IL-22 is required for Th17 cellmediated pathology in a mouse model of psoriasis-like skin inflammation. J Clin Invest 2008; 118:597-607.

65. Chiricozzi A, Su_arez-Fari nas M, Fuentes-Duculan J, Cueto I, Li K, Tian S, et al. Increased expression of interleukin-17 pathway genes in nonlesional skin of moderate-to-severe psoriasis vulgaris. Br J Dermatol 2016;174:136-45. 


\section{CHAPTER 5}

66. Nair RP, Duffin KC, Helms C, Ding J, Stuart PE, Goldgar D, et al. Genome-wide scan reveals association of psoriasis with IL-23 and NF-kB pathways. Nat Genet 2009;41:199-204.

67. Dudakov JA,HanashAM, van den BrinkMR. Interleukin-22: immunobiology and pathology. Annu Rev Immunol 2015;33:747-85.

68. Peters $\mathrm{CP}$, Mj€osberg JM, Bernink JH, Spits $\mathrm{H}$. Innate lymphoid cells in inflammatory bowel diseases. Immunol Lett 2016;172: 124-31.

69. Buonocore S, Ahern PP, Uhlig HH, Ivanov II, Littman DR, Maloy KJ, et al. Innate lymphoid cells drive interleukin-23-dependent innate intestinal pathology. Nature 2010;464:1371-5.

70. Geremia A, Arancibia-C_arcamo CV, Fleming MP, Rust N, Singh B, Mortensen NJ, et al. IL-23responsive innate lymphoid cells are increased in inflammatory bowel disease. J Exp Med $2011 ; 208: 1127-33$.

71. Sawa S, Lochner M, Satoh-Takayama N, Dulauroy S, Berard M, Kleinschek M, et al. RORgt+ innate lymphoid cells regulate intestinal homeostasis by integrating negative signals from the symbiotic microbiota. Nat Immunol 2011;12:320-6.

72. Hepworth MR, Fung TC, Masur SH, Kelsen JR, McConnell FM, Dubrot J, et al. Group 3 innate lymphoid cells mediate intestinal selection of commensal bacteria-specific CD4+ T cells. Science 2015;348:1031-5.

73. Reinhardt A, Yevsa T, Worbs T, Lienenklaus S, Sandrock I, Oberdorfer L, et al. Interleukin-23dependent $\mathrm{g} / \mathrm{d}$ T cells produce interleukin-17 and accumulate in the enthesis, aortic valve, and ciliary body in mice. Arthritis Rheumatol 2016;68:2476-86.

74. Ono T, Okamoto K, Nakashima T, Nitta T, Hori S, Iwakura Y, et al. IL-17-producing gd T cells enhance bone regeneration. Nat Commun 2016;7:10928.

75. Cuthbert R, Fragkakis E, Millner P, Dunsmuir R, El-Sherbiny Y, McGonagle D. A1.26 innate lymphoid cells are present at normal human enthesis providing a potential mechanism for spondyloarthropathy pathogenesis. Ann Rheum Dis 2016;75 Suppl 1:A11.

76. Tang F, Sally B, Ciszewski C, Abadie V, Curran SA, Groh V, et al. Interleukin 15 primes natural killer cells to kill via NKG2D and CPLA2 and this pathway is active in psoriatic arthritis. PLoS One 2013;8:e76292.

77. Nielsen N, Pascal V, Fasth AE, Sundstrom Y, Galsgaard ED, Ahern D, et al. Balance between activating NKG2D, DNAM-1, NKp44 and NKp46 and inhibitory CD94/NKG2A receptors determine natural killer degranulation towards rheumatoid arthritis synovial fibroblasts. Immunology 2014;142:581-93.

78. Carrega P, Loiacono F, Di Carlo E, Scaramuccia A, Mora M, Conte R, et al. NCR1/LC3 concentrate in human lung cancer and associate with intratumoral lymphoid structures. Nat Commun 2015;6:8280.

79. Białoszewska A, Baychelier F, Niderla-Bielinska J, Czop A, Debre P, Vieillard V, et al. Constitutive expression of ligand for natural killer cell NKp44 receptor (NKp44L) by normal human articular chondrocytes. Cell Immunol 2013;285:6-9.

80. Baeten D, Sieper J, Braun J, Baraliakos X, Dougados M, Emery P, et al. Secukinumab, an interleukin-17A inhibitor, in ankylosing spondylitis. N Engl J Med 2015;373:2534-48. 
81. Sherlock JP, Joyce-Shaikh B, Turner SP, Chao CC, Sathe M, Grein J, et al. IL-23 induces spondyloarthropathy by acting on ROR-gt+ CD3+CD4-CD8- entheseal resident $\mathrm{T}$ cells. Nat Med 2012;18:1069-76.

82. Ciccia F, Accardo-Palumbo A, Alessandro R, Rizzo A, Principe S, Peralta S, et al. Interleukin-22 and interleukin-22-producing NKp44+ natural killer cells in subclinical gut inflammation in ankylosing spondylitis. Arthritis Rheum 2012;64:1869-78.

83. Ciccia F, Guggino G, Rizzo A, Saieva L, Peralta S, Giardina A, et al. Type 3 innate lymphoid cells producing IL-17 and IL-22 are expanded in the gut, in the peripheral blood, synovial fluid and bone marrow of patients with ankylosing spondylitis. Ann Rheum Dis 2015;74:1739-47.

84. Taurog JD, Richardson JA, Croft JT, Simmons WA, Zhou M, Fernandez-Sueiro JL, et al. The germfree state prevents development of gut and joint inflammatory disease in HLA-B27 transgenic rats. J Exp Med 1994;180:2359-64.

85. Asquith MJ, Stauffer P, Davin S, Mitchell C, Lin P, Rosenbaum JT. Perturbed mucosal immunity and dysbiosis accompany clinical disease in a rat model of spondyloarthritis. Arthritis Rheumatol 2016;68:2151-62.

86. Zhou X, Wang J, Zou H, Ward MM, Weisman MH, Espitia MG, et al. MICA, a gene contributing strong susceptibility to ankylosing spondylitis. Ann Rheum Dis 2014;73:1552-7.

87. Duffin R, Connor RA, Crittenden S, Forster T, Yu C, Zheng X, et al. Prostaglandin E2 constrains systemic inflammation through an innate lymphoid cell-IL-22 axis. Science 2016;351:1333-8.

88. Magri G, Cerutti A. Role of group 3 innate lymphoid cells in antibody production. Curr Opin Immunol 2015;33:36-42.

89. Withers DR, Gaspal FM, Mackley EC, Marriott CL, Ross EA, Desanti GE, et al. Cutting edge: lymphoid tissue inducer cells maintain memory CD4 T cells within secondary lymphoid tissue. J Immunol 2012;189:2094-8.

90. Noort AR, van Zoest KP, van Baarsen LG, Maracle CX, Helder B, Papazian N, et al. Tertiary lymphoid structures in rheumatoid arthritis: NF-kB-inducing kinase-positive endothelial cells as central players. Am J Pathol 2015;185:1935-43.

91. Rodriguez-Carrio J, Hahnlein JS, Ramwadhdoebe TH, Semmelink JF, Choi IY, van Lienden KP, et al. Altered innate lymphoid cell subsets in human lymph node biopsy specimens obtained during the at-risk and earliest phases of rheumatoid arthritis. Arthritis Rheumatol 2017;69:706.

92. Cortez VS, Cervantes-Barragan L, Robinette ML, Bando JK, Wang Y, Geiger TL, et al. Transforming growth factor-b signaling guides the differentiation of innate lymphoid cells in salivary glands. Immunity 2016;44:1127-39.

93. Ciccia F, Guggino G, Rizzo A, Ferrante A, Raimondo S, Giardina A, et al. Potential involvement of IL-22 and IL-22- producing cells in the inflamed salivary glands of patients with Sjogren's syndrome. Ann Rheum Dis 2012;71:295-301.

94. Nocturne G, Mariette X. Advances in understanding the pathogenesis of primary Sjogren's syndrome. Nat Rev Rheumatol 2013;9:544-56. 


\section{CHAPTER 5}

95. Magri G, Miyajima M, Bascones S, Mortha A, Puga I, Cassis L, et al. Innate lymphoid cells integrate stromal and immunological signals to enhance antibody production by splenic marginal zone B cells. Nat Immunol 2014;15:354-64.

96. Rusakiewicz S, Nocturne G, Lazure T, Semeraro M, Flament C, Caillat-Zucman S, et al. NCR3/NKp30 contributes to pathogenesis in primary Sjogren's syndrome. Sci Transl Med 2013;5:195ra96.

97. Wohlfahrt T, Usherenko S, Englbrecht M, Dees C, Weber S, Beyer C, et al. Type 2 innate lymphoid cell counts are increased in patients with systemic sclerosis and correlate with the extent of fibrosis. Ann Rheum Dis 2016;75:623-6.

98. Reefman E, Kuiper H, Limburg PC, Kallenberg CG, Bijl M. Type I interferons are involved in the development of ultraviolet B-induced inflammatory skin lesions in systemic lupus erythaematosus patients. Ann Rheum Dis 2008;67:11-18.

99. Zhang Z, Cheng L, Zhao J, Li G, Zhang L, Chen W, et al. Plasmacytoid dendritic cells promote HIV-1-induced group 3 innate lymphoid cell depletion. J Clin Invest 2015;125:3692-703.

100. Duerr CU, McCarthy CD, Mindt BC, Rubio M, Meli AP, Pothlichet J, et al. Type I interferon restricts type 2 immunopathology through the regulation of group 2 innate lymphoid cells. Nat Immunol 2016;17:65-75.

101. Bijl M, Kallenberg CG. Ultraviolet light and cutaneous lupus. Lupus 2006;15:724-7.

102. Gimblet C, Loesche MA, Carvalho L, Carvalho EM, Grice EA, Artis D, et al. IL-22 protects against tissue damage during cutaneous Leishmaniasis. PLoS One 2015;10:e0134698.

103. Avitabile S, Odorisio T, Madonna S, Eyerich S, Guerra L, Eyerich K, et al. Interleukin-22 promotes wound repair in diabetes by improving keratinocyte pro-healing functions. J Invest Dermatol 2015;135:2862-70.

104. Mendez-Flores S, Hernandez-Molina G, Enrıquez AB, Faz-Munoz D, Esquivel Y, Pacheco-Molina $C$, et al. Cytokines and effector/regulatory cells characterization in the physiopathology of cutaneous lupus erythematous: a cross-sectional study. Mediators Inflamm 2016;2016:7074829.

105. Clements P, Lachenbruch P, Seibold J, White B, Weiner S, Martin R, et al. Inter and intraobserver variability of total skin thickness score (modified Rodnan TSS) in systemic sclerosis. J Rheumatol 1995;22:1281-5.

106. Brkic $Z$, van Bon $L$, Cossu M, van Helden-Meeuwsen CG, Vonk MC, Knaapen $H$, et al. The interferon type I signature is present in systemic sclerosis before overt fibrosis and might contribute to its pathogenesis through high BAFF gene expression and high collagen synthesis. Ann Rheum Dis 2016;75:1567-73.

107. Roan F, Stoklasek TA, Whalen E, Molitor JA, Bluestone JA, Buckner JH, et al. CD4+ group 1 innate lymphoid cells (ILC) form a functionally distinct ILC subset that is increased in systemic sclerosis. J Immunol 2016;196:2051-62.

108. Hams E, Armstrong ME, Barlow JL, Saunders SP, Schwartz C, Cooke G, et al. IL-25 and type 2 innate lymphoid cells induce pulmonary fibrosis. Proc Natl Acad Sci U S A 2014;111:367-72. 
109. Hams E, Bermingham R, Fallon PG. Macrophage and innate lymphoid cell interplay in the genesis of fibrosis. Front Immunol 2015; 6:597.

110. Lei L, Zhao C, Qin F, He ZY,Wang X, Zhong XN. Th17 cells and IL-17 promote the skin and lung inflammation and fibrosis process in a bleomycin-induced murine model of systemic sclerosis. Clin Exp Rheumatol 2016;34 Suppl 100:14-22.

111. Triggianese P, Conigliaro P, Chimenti MS, Biancone L, Monteleone G, Perricone R, et al. Evidence of IL-17 producing innate lymphoid cells in peripheral blood from patients with enteropathic spondyloarthritis. Clin Exp Rheumatol 2016;34:1085-93.

112. Braudeau C, Amouriaux K, N_eel A, Herbreteau G, Salabert N, Rimbert M, et al. Persistent deficiency of circulating mucosalassociated invariant T (MAIT) cells in ANCA-associated vasculitis. J Autoimmun 2016;70:73-9.

113. Bjorklund AK, Forkel M, Picelli S, Konya V, Theorell J, Friberg D, et al. The heterogeneity of human CD127+ innate lymphoid cells revealed by single-cell RNA sequencing. Nat Immunol 2016;17:451-60.

114. Yu Y, Tsang JC, Wang C, Clare S, Wang J, Chen X, et al. Single-cell RNA-seq identifies a PD-1 hi ILC progenitor and defines its development pathway. Nature 2016;539:102-6.

115. Huang T, Hazen M, Shang Y, Zhou M, Wu X, Yan D, et al. Depletion of major pathogenic cells in asthma by targeting CRTh2. JCl Insight 2016;1:e86689. 


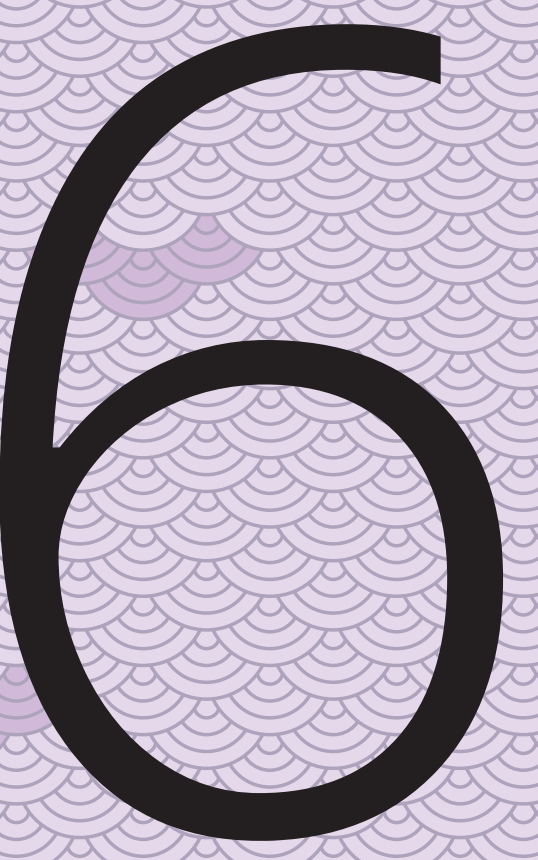




\title{
CHAPTER 6
}

\section{Tissue-resident memory CD8+ T cells from skin differentiate psoriatic arthritis from psoriasis}

\begin{abstract}
Authors:
Emmerik F. Leijten ${ }^{1,2}$ MD, Tessa S. van Kempen ${ }^{1,2}$ PhD, Michel A. Olde Nordkamp² BSc, Juliette N. Pouw ${ }^{1,2}$ MD, Nienke J. Kleinrensink ${ }^{1,3}$ MD, Nanette L. Vincken ${ }^{1,2}$ MD, Jorre Mertens ${ }^{1,2}$ MD PhD, Deepak M.W. Balak MD PhD, Fleurieke H. Verhagen 2,5 MD PhD, Sarita A. Hartgring ${ }^{1,2}$ PhD, Erik Lubberts ${ }^{6,7}$ PhD, Janneke Tekstra' MD PhD, Aridaman Panditi, PhD, Timothy R. Radstake ${ }^{1,2}$ * MD PhD, Marianne Boes ${ }^{2,8}$ * PhD *contributed equally
\end{abstract}

Affiliations:

1. Department of Rheumatology and Clinical Immunology, UMC Utrecht, Utrecht, Netherlands 2. Center for Translational Immunology, UMC Utrecht, Utrecht, Netherlands 3. Department of Radiology, UMC Utrecht, Utrecht, Netherlands 4. Department of Dermatology, UMC Utrecht, Utrecht, Netherlands 5. Department of Ophthalmology, UMC Utrecht, Utrecht, Netherlands 6. Department of Rheumatology, Erasmus Medical Center, University Medical Center, Rotterdam, The Netherlands.

7. Department of Immunology, Erasmus Medical Center, University Medical Center, Rotterdam, The Netherlands

8. Department of Pediatrics, UMC Utrecht, Utrecht, Netherlands

Published in:

Arthritis and Rheumatology. 2021 Jan 16. Online ahead of print doi: 10.1002/art.41652 


\section{Abstract}

Objectives: Studies that compare immune cell phenotype and function in psoriatic arthritis (PsA) versus psoriasis limited to cutaneous involvement (Pso) are scarce, yet critical to understand the pathogenesis of PsA.

Methods: First, in-depth immunophenotyping of different T cell and Dendritic cell subsets was performed in PsA, Pso, axial spondyloarthritis patients and healthy controls. Subsequently, we analyzed cells from peripheral blood, synovial fluid, and skin biopsies using flow cytometry, alongside high-throughput transcriptome analyses and functional assays on the specific cell population differentiating PsA from Pso.

Results: As compared to HC, the peripheral blood of PsA was characterized by an increase of regulatory CD4 T cells and IL-17A \& IL-22 co-producing CD8+ T cells. One population specifically differentiated PSA from Pso: CCR10+ CD8+ T cells, being enriched in PSA. CCR10+ CD8+ T cells were phenotypically DNAM1 ${ }^{\text {hi }}$, effector memory cells that co-expressed skin homing receptors CCR4 and CLA. CCR10+ CD8+ T cells were detected under inflammatory and homeostatic conditions in skin, but were not enriched in synovial fluid. Gene profiling further revealed that CCR10+ CD8+ T cells expressed GATA3, FOXP3, and core transcriptional signature of tissue-resident memory $T$ cells, including CD103. Specific genes (including RORC, IFNAR1, ERAP1) were upregulated in PSA compared to PSO. CCR10+ CD8+ were endowed with Tc2/22-like cytokine profile, lacked cytotoxic potential, and displayed overall regulatory function.

Conclusion: Tissue-resident memory CD8+ T cells derived from the skin are enhanced in the circulation of PsA as compared to Pso. This may indicate aberrances in cutaneous tissue homeostasis contribute to arthritis development. 


\section{Introduction}

New critical insights into the pathogenesis of psoriasis (Pso) and psoriatic arthritis (PsA) have been made in recent years, including the role of the IL-23/IL-17 axis $(1,2)$. This finding further propelled the development of novel drugs with potential to vastly improve psoriasis, but their strength at halting arthritis is less impressive. A clearer understanding of the pathogenesis of PsA could pinpoint the development of therapeutics capable of resolving arthritis.

The prevalence of PsA in patients with Pso is approximately $20 \%(2)$ and in the most simple form presents with arthritis in a patient with a history of psoriasis. However, the relationship between skin and joint manifestations encompass a spectrum: patients can have severe psoriasis without musculoskeletal complaints, while others have minimal psoriasis and severe arthritis.

This raised the question whether these diseases are part of a single spectrum or are separate entities $(3,4)$. More specifically, it is currently unknown whether immunological processes in the skin and the joint are directly related. One possibility is that immunological processes in skin and joint occur in parallel, but independently from each other. Some arguments for this hypothesis are the aforementioned clinical disease heterogeneity of PSA and that some tissuesites respond better to specific treatment targets(2). Also, observations that local tissue damage (e.g. koebner phenomenon) can trigger local inflammation fit this concept(5).

Another possibility is that the pathophysiological processes are directly linked and occur sequentially. Supporting this hypothesis is that Pso itself is a strong risk factor for developing arthritis and that Pso typically precedes the onset of arthritis by many years. In this scenario, immune cells, cytokines and/or other mediators induced by skin inflammation could trigger a second hit at the joint. Indeed, soluble factors have been shown capable of inducing models of spondyloarthritis(6), potentially sparking responses of resident, innate lymphocytes at the musculoskeletal sites(7). While the role of different immune cells in skin and joint has been described(1), there is a scarcity of reports that explicitly compare immune cells between Pso and PsA(8-11). In this study we first performed in-depth immunophenotyping in patients with Pso and PSA (free of DMARDs and matched for skin disease activity measured by psoriasis area and severity index(PASI)), which was followed by phenotypic, transcriptomic and functional investigation of the specific CD8+ T cell subset that best distinguished PsA from Pso. 


\section{Patients and Methods}

\section{Study cohort and samples}

The study was performed at the Department of Rheumatology and Clinical Immunology, University Medical Centre Utrecht (UMCU), in compliance with the Helsinki principles and approved by the institutional review board. All patients signed written informed consent before participation. Pso patients had a dermatologist-confirmed diagnosis of psoriasis. All Pso patients underwent clinical evaluation to exclude concomitant PsA. PsA patients fulfilled CIASsification of Psoriatic ARthritis (CASPAR) criteria(12). Axial Spondyloarthritis (AxSpA) patients met the Assessment of SpondyloArthritis International Society classification criteria(13) without concomitant psoriasis.

In the first phase, the frequency of T cells and dendritic cells/subsets in peripheral blood mononuclear cells (PBMC) from PsA ( $n=21)$ was compared to healthy control $(H C)(n=20)$, Pso $(n=21)$, and Axial Spondyloarthritis (AxSpA, $n=16)$. Pso and PsA were matched for key clinical parameters, including PASI (Sup Table S1). In the second phase, we specifically investigated the properties of CCR10+ CD8+ T cells, for which additional samples were collected: PBMC (HC n=32; Pso $n=17 ;$ PsA n=18), synovial fluid (PsA n=8) and skin biopsies (PsA n=6; Pso $n=8$ ). With the exception of synovial fluid samples, all samples were obtained from patients free of diseasemodifying anti-rheumatic drugs at the time of participation.

\section{Sample collection}

PBMC were isolated according to density centrifugation using Ficoll-Paque Plus (GE Healthcare) from lithium-heparinized venous blood and first stored in liquid nitrogen. Four millimeter punch biopsies from lesional psoriatic skin sites (donor-dependent lesional site) and non-lesional skin sites (always dorsal thorax) were taken and placed in phosphate-buffered saline on ice before further processing. The skin biopsies directly underwent mechanical and tissue digestion according to manufacturer protocol (Whole Skin Dissociation Kit, human, Miltenyi Biotec), after which flow cytometry was performed on the freshly digested skin biopsies. Synovial fluid mononuclear cells were isolated according to density centrifugation using Ficoll-Paque Plus (GE Healthcare) gradient procedure and first stored in liquid nitrogen.

\section{Flow cytometry}

Four different flow cytometry panels were used to identify and enumerate in thawed PBMC the relative frequency of a broad range of $\mathrm{T}$ cell and dendritic cell subsets, as depicted in Fig S1-S4, by using a standardized flow-cytometry 
protocol, as previously described in detail(14). Antibodies used are listed in Table S2. Fixation and permeabilization solution was used according to manufacturer instructions (eBioscience, San Diego, CA, USA) for intra-cellular antibody staining. Fluorescence-minus one was used as negative control for determining manual gating strategy. Flow cytometry data acquisition was done on BD LSR Fortessa ${ }^{\mathrm{TM}}$ Cell analyzer and flow cytometric cell sorting was done on BD FACSAria ${ }^{\mathrm{TM}}$ III (BD Bioscience, San Jose, CA, USA). Different subsets of CD8+ T cells were flow-sorted based on expression of CCR10 and/or CCR4 (Fig S5) for proliferation suppression assays and RNA-sequencing.

\section{Functional assays}

PBMC were re-stimulated for four hours in culture medium (RPMI-1640 with 10\% Fetal calf serum) with Phorbol 12-myristate 13-acetate (PMA), Ionomycin calcium salt and BD GolgiPlug (BD Biosciences, San Jose, CA, USA) at 37 degrees Celsius for quantification of intra-cellular cytokine production by flow cytometry.

Proliferation suppression assays were performed in accordance to established protocols for regulatory T cells(15). For this assay, fresh PBMC were isolated from HC $(n=6)$ from which "regulatory-type" cells and "target cells" were derived. The "regulatory-type" cells were different flow-sorted CD8+ T cells subsets (Fig S5). The target cells were CellTrace ${ }^{\mathrm{TM}}$ Violet (Thermo Fisher) labelled autologous PBMC. The "regulatory-type" cells and target cells were resuspended in culture medium (RPMI-1640 with 10\% Fetal calf serum) and co-cultured at a ratio of 1:2 cells (respectively) in anti-CD3 coated plates for four days. The readout was the percent of proliferated target cells. As reference for the readout, regulatory CD4 T cells (CD3+CD4+ CD25 ${ }^{\mathrm{Hi}}$ CD127-) and effector CD4 T cells (CD3+CD4+ CD25-CD127Hi) were flow-sorted from the same donors and taken along in each experiment, which indicated the suppression assay worked (data not shown).

\section{RNA-sequencing}

From thawed PBMC ( $\mathrm{HC} n=8$; Pso $n=6$; PsA $n=6)$ distinct CD8+ T cell subsets were flow-sorted (Fig S5). Cells were lysed in RLTplus buffer in the presence of beta-mercaptoethanol (final concentration 1\%) and RNA was isolated according to manufacturer protocol (Qiagen Universal Kit). In total, 57 samples were used for analysis (all passed internal quality control checks). RNA sequencing was performed using an Illumina HiSeq 4000 sequencer (Illumina) (Paired-End, 150 bp) at GenomeScan, Leiden using standard manufacturer's protocols. FastQC was used to check the quality of the raw reads obtained from RNAseq (https:// www.bioinformatics.babraham.ac.uk/projects/fastqc/). STAR aligner was used to align the reads to the human genome (GRCh38 build 79)(16,17). HTSeq was used 
to obtain read counts for each annotated gene(18). Differential gene expression (DGEs) was calculated using Bioconductor/R package DESeq2(19). Wald's test was used to identify DGEs between each condition (HC, Pso, and PsA) and cell subset (Fig S5) pair. Variance stabilizing transformation was applied to the raw read count data to obtain normalized gene counts (variance stabilized data or VSD), which were used for subsequent plotting. The heatmap, PCA and violin plots were plotted using R.

\section{Statistical analysis}

Categorical variables were compared using Chi-squared tests and group differences were compared using Mann-Whitney $U$ test or independent samples T-test (based on normality distribution). Group differences were compared using the Wilcoxon signed-rank test for paired samples. Spearman's rank correlation tested correlation between clinical parameters and flow cytometry results.

Flow cytometry data were analyzed using Flowjo software (TreeStar inc. San Carlos, CA, USA). Statistical analysis and visual representation of the data was performed using SPSS version (v25) and Graphpad Prism software (v7.0). Heatmap of flow cytometry results was made using MetaboAnalyst (v4.0) using Ward clustering algorithm and autoscaling of features (20). Venn diagrams were made using Venny (v2.1)(21). Unless otherwise stated, a $p$-value of $<0.05$ was considered statistically significant.

\section{Results}

\section{Higher frequency of circulating CD8+ CD45RO+ CCR10+ T cells in psoriatic arthritis compared to psoriasis}

We compared the frequency of T cell and dendritic cell subsets in peripheral blood in PsA to HC, Pso and AxSpA, using our standardized immunophenotyping panels (Table S1; Fig S1-S4). The results of immunophenotyping shown in a heatmap in Figure 1A support an overall similarity in PBMC subsets of Pso and PsA. The CD8+ CD45RO+ CCR10 + subset was the only cell population significantly different between PsA and Pso, being elevated in patients with PsA $(p<0.05)$ (Fig 1B). As compared to HC, patients with PsA had increased frequency of CCR10+ CD8+ T cells, IL-17A+IL-22+ CD8+ T cells and regulatory CD4+ T cells. Patients with PSA also had reduced frequency of plasmacytoid DCs and CD161+CCR6+ CD8+ T cells (MAIT-like cells) as compared to HC (Fig 1B-F). Considering the scarcity of reports examining CCR10 expression on CD8+ T cells(22-24), we subsequently set out to further characterize the phenotype, origin and function of CCR10-expression on CD8 T cells in general and in relationship to PsA. 
A

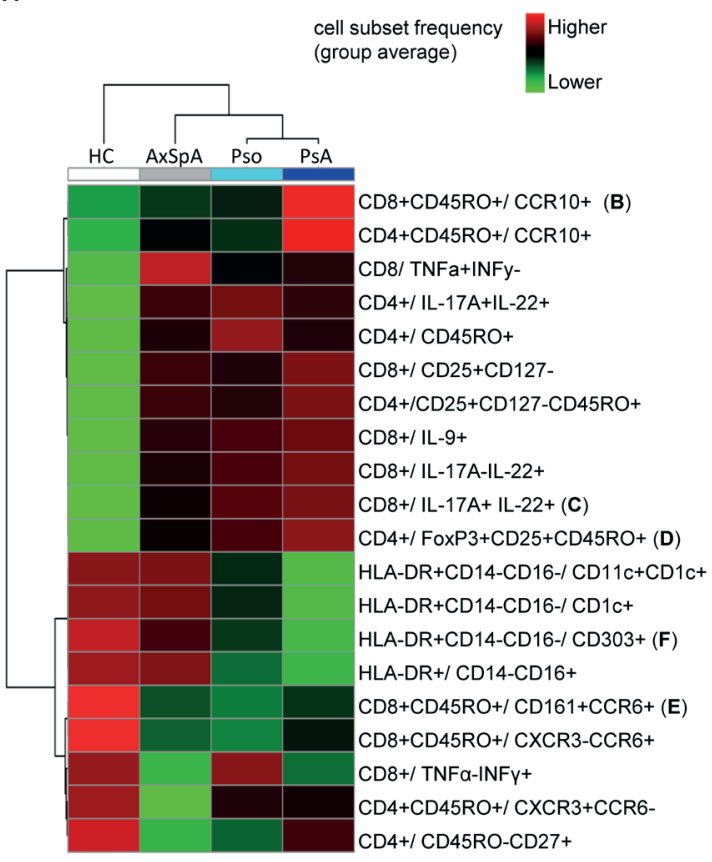

C IL-17A+IL-22+

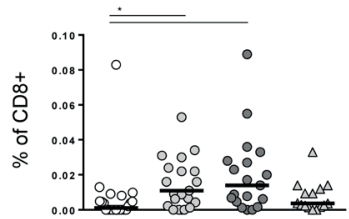

D

FoxP3+CD25+CD45RO+

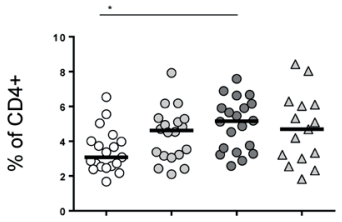

B
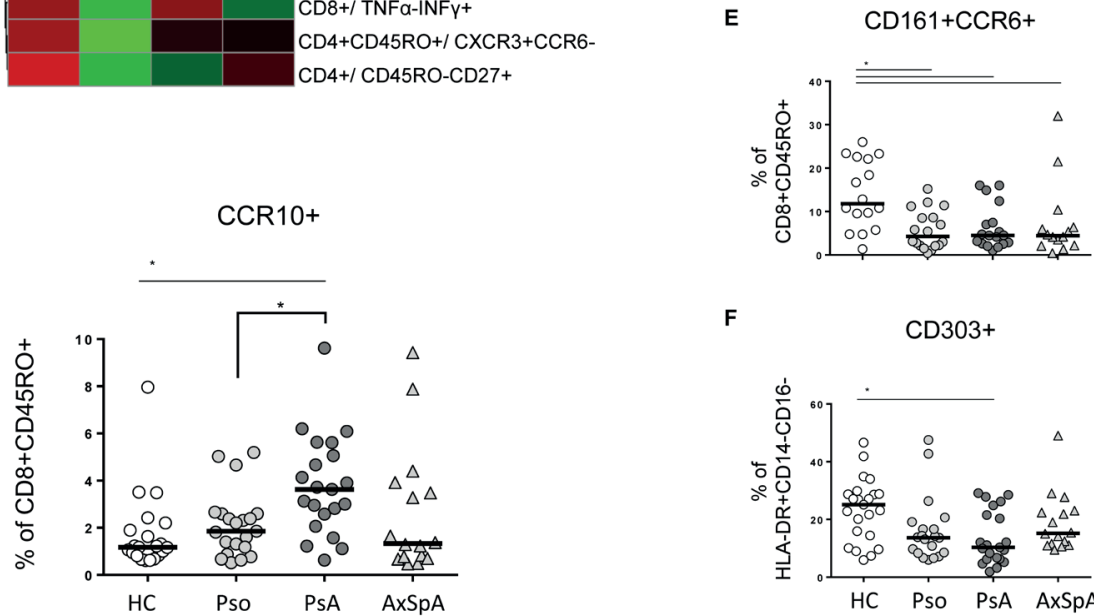

$\mathbf{F}$

CD303+

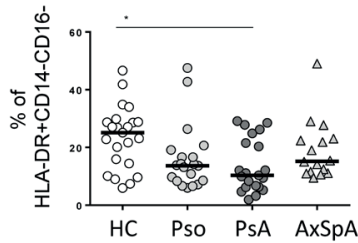

Fig 1. Phenotype and function of circulating immune subsets in psoriatic arthritis

Frequency (\%) of T cell and dendritic cell subsets from peripheral blood mononuclear cells (PBMC): (A) Overview of the flow-cytometry features that best distinguished the different groups (top 20 features as based on ANOVA) is visualized in a heatmap, where cell subset frequency is displayed as group average (autoscaling of features). (B) CCR10+ within CD8+CD45RO+; (C) IL-17A+IL-22+ within CD8+; (D) FoxP3+CD25+CD45RO+ within CD4+; (E) CD161+CCR6+ within CD8+CD45RO+; (F) CD303+ within HLADR+CD14-CD16-. (B) Graphs depict median. Asterisk indicates $\mathrm{p}<0.05$. 


\section{CCR10 expression on CD8+ T cells marks a memory, DNAM-1 ${ }^{\text {high }}$ phenotype}

We first went back to examine the phenotype of CD8+ T cells that expressed CCR10 (not using CD45RO+ as prerequisite). As expected, directly gating on CCR10+ CD8 $T$ cells the majority were either classified as central memory (CM) or effector memory (EM) (Fig S6 panel A-B). CCR10 expression was significantly elevated on both CM and EM subsets in patients with PSA (Fig S6 panel C-D). CCR10+ CD8+ T cells were also enriched for CCR6 co-expression, while the expression of CD45RO, CD27, CCR6, CXCR3 was similar across patient groups with respect to the CCR10+ CD 8 T cells (not shown).

We broadly screened additional CD8 T cell marker and found CCR10+ CD8+ T cells co-expressed DNAX Accessory Molecule-1 (DNAM-1) (Fig 2A). DNAM-1 is an activating receptor and TIGIT an inhibitory receptor on T cells, that compete for binding to CD155, an immunoglobulin-like adhesion molecule. Based on DNAM-1 and TIGIT, distinct co-expression patterns were detected on CD8+ T cells (Fig 2B). Overall, CCR10+ CD8+ T cells were typically DNAM-1 ${ }^{\text {hi }}$ (Fig 2C), but with less TIGIT co-expression in patients with PsA (Fig 2D).

A

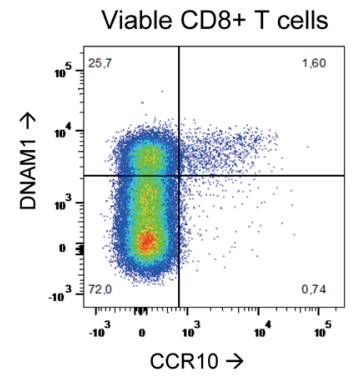

C

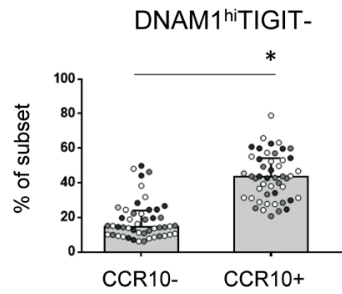

Viable CD8+ T cells

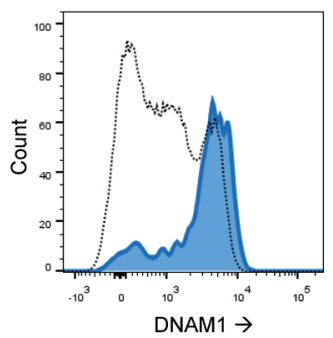

DNAM $1{ }^{\text {hiTIGIT+ }}$

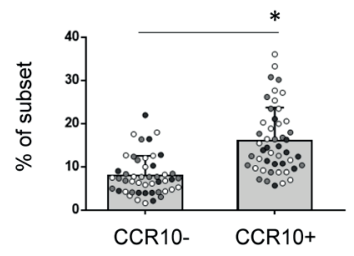

B Viable $\mathrm{CD} 8+\mathrm{T}$ cells

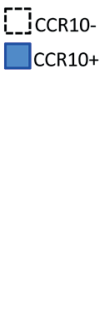

DNAM1-TIGIT+

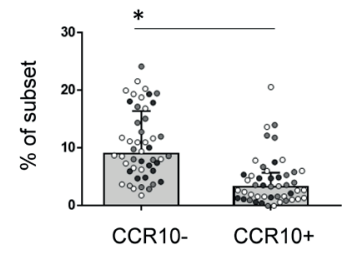


D

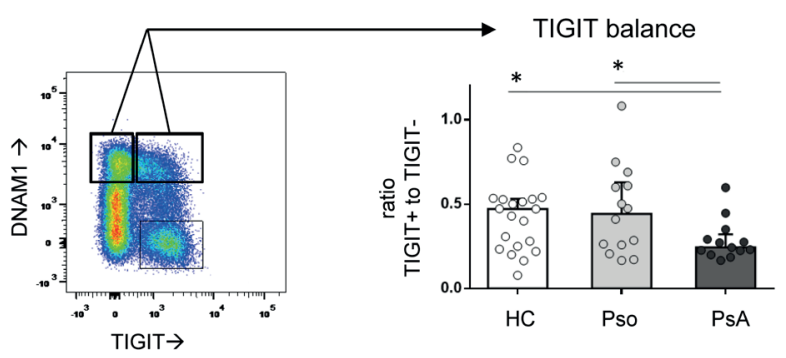

Fig 2. CCR10+ CD8+ T cells are prototypically DNAM-1 high. (A) CCR10+ CD8+ T cells co-expressed high levels of DNAM-1. (B) Distinct population of CD8+ T cells are distinguishable based on DNAM-1 and TIGIT, including DNAM-1 ${ }^{\text {hiTIGIT- (top left), DNAM-1 }}$ hiTIGIT+ (top right) and DNAM-1-TIGIT+ (bottom right). (C) The

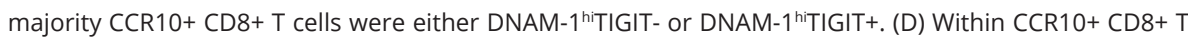
cells with high expression for DNAM-1, the TIGIT co-expression was reduced in patients with PSA. Graphs depict median+IQR. White circles indicate HC, grey circles indicate Pso, black circles indicate PsA. Asterisk indicates $\mathrm{p}<0.05$.

\section{CCR10+ CD8+ T cells are enriched in skin, not joint}

To investigate their tissue origin, we enumerated the frequency of CCR10+ CD8+ T cells in skin biopsies and in synovial fluid mononuclear cells (SFMC). The frequency of CCR10+CD8+ T cells was significantly higher in skin as compared to paired PBMC. In contrast, there was no enrichment of CCR10+ CD8+ T cells in SFMC samples compared to (non-paired) PBMC (Fig 3A; Fig S7). Previous reports indicate CCR10 is a chemokine receptor found on skin-tropic T cells. We confirmed that the majority of circulating CCR10+ CD8+ T cells co-expressed the skin-homing markers CCR4 and CLA (Fig 3B-C). Conversely, CCR10+ CD8+ T cells did not coexpress the integrin $\beta$ 7, a marker associated with gut-homing properties (Fig 3D).

Next, we checked if CCR10+ CD8+ T cell frequency was related to measures of disease activity. The frequency of CCR10+ CD8+ T cells in PBMCs was not related to tender or swollen joint count (not shown). Further, we detected a stable frequency of CCR10+ CD8+ T cells in PBMC obtained from a Pso patient before onset and after onset of PSA. The frequency of CCR10+ CD8+ T cells was not higher in the SFMC compartment at disease onset from this patient (Fig $\mathbf{3 E}$ ). These results indicate the joint compartment is an unlikely source of CCR10+ CD8+ T cells.

As expected, we found that the lesional skin contained a much larger absolute number of CD8+ T cells than non-lesional skin (Fig S7). However, the fraction of CD8+ T cells expressing CCR10 was either similar or lower in lesional skin compared to non-lesional skin (Fig 3A and 3F). The frequency of CCR10+ CD8+ T cells in PBMCs was not related to PASI (not shown). CD103 expression, a marker 
of tissue retention, was significantly elevated on CCR10+ CD8+ T cells from both PBMC and skin (Fig S8). In summary, CCR10 is a marker for skin-tropic CD8+ T cells in circulation and enriched in both lesional and non-lesional psoriatic skin, but not enriched in synovial fluid.

A

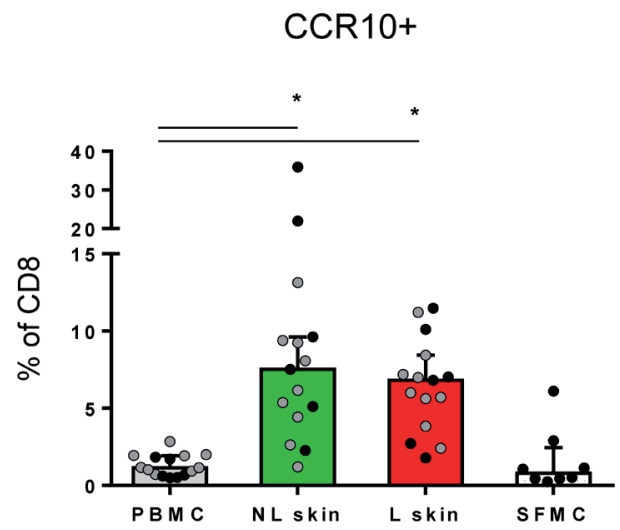

B

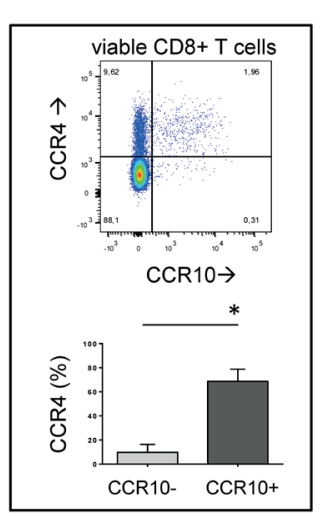

C

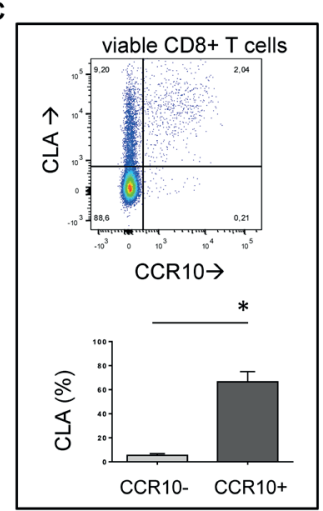

D

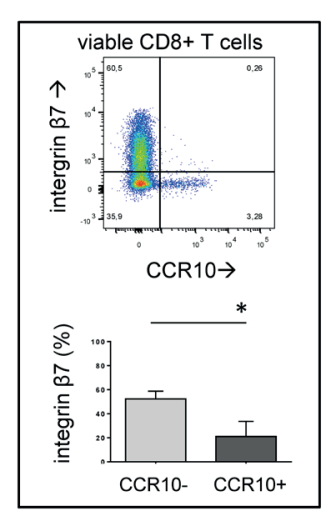


$\mathbf{E}$ Pre-onset-PsA
PBMC sample

First-onset-PsA PBMC sample

First-Onset-PsA SFMC sample viable CD8+ T cells

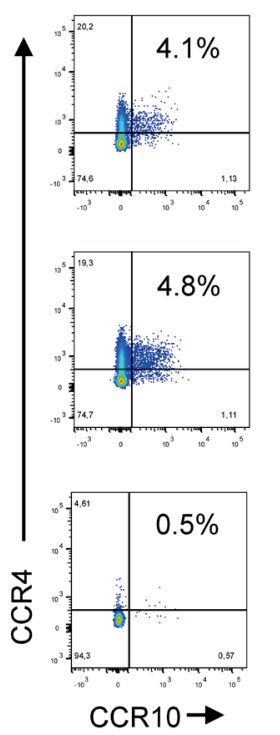

F viable CD8+ T cells

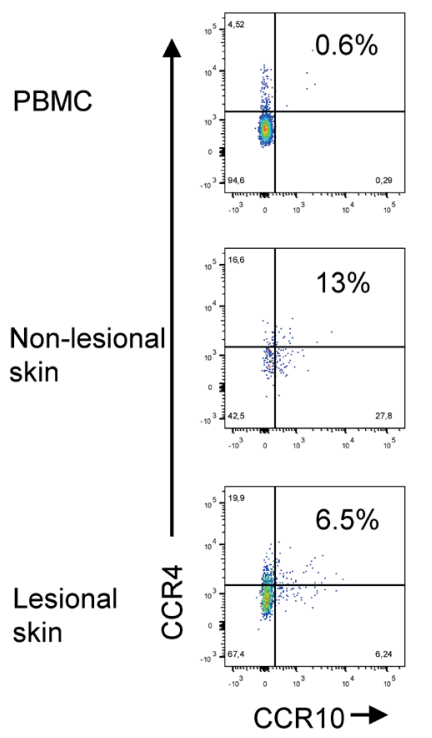

Fig 3. CCR10+ CD8+ T cells are enriched in skin, but not in synovial fluid

(A) CCR10+ CD8+ T cell frequency in paired non-lesional (NL) skin, lesional (L) skin and PBMC, as well as (non-paired) SFMC. Grey circles indicate Pso, black circles indicate PSA. (B-D) CCR4, CLA, and integrin beta-7 expression in CCR10+ versus CCR10- CD8+ T cells. (E) CCR10+ CD8+ T cell frequency in PBMC from one psoriasis patient before onset of PSA and from the same patient in PBMC and SFMC at first onset of PsA. (F) CCR10+ CD8+ T cells frequency in paired NL, L and PBMCs from a PsA patient. Graphs depict median+IQR. Asterisk indicates $\mathrm{p}<0.05$.

\section{CCR10+ CD8+ T cells express GATA3, FOXP3 and tissue- resident memory profile}

We next performed transcriptome analyses of circulating CD8+ T cells from HC, Pso, and PsA patients. Three populations of viable CD8+ T cells were sorted: CCR10+, CCR4+, and CCR10-CCR4-(Fig 4A)(gating depicted in Fig S5). We found that the CCR10+ subset and CCR4+ subset were largely overlapping, while both were very distinct from the CCR10-CCR4- fraction (Fig 4B; Fig 4C). As compared to the CCR10-CCR4- fraction, the CCR10+ subset was different with respect to numerous well-characterized genes, which include the upregulation of GATA3, CCR8, IL4R, and CD44 (Fig S9). In addition, the CCR10+ subset had high expression of FOXP3 and lacked expression of genes associated with cytotoxic potential (GZMB and PRF1) (Fig 4C). Moreover, CCR10+ CD8+ T cells displayed a prototypical gene expression pattern resembling tissue-resident memory T cells (Trm): high expression of ITGAE (CD103), CD69, CCR8, CD44 and low expression of KLRG1 and CX3CR1 (Fig 4D). 
A

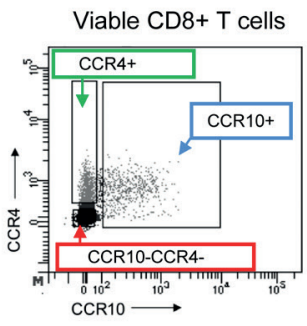

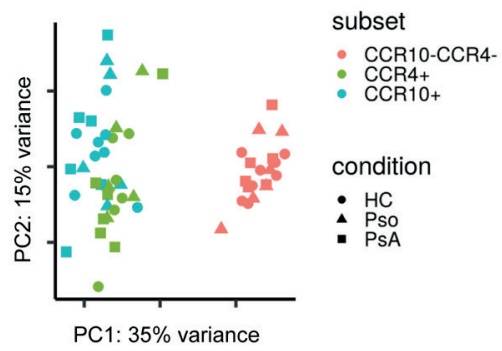

D

CCR10+ subset shares core transcriptional profile of Trm Upregulated in Trm: CD69, ITGAE, IL7R, CCR8, CD44 Downregulated in Trm: CX3CR1, KLRG1, CCR7, SELL
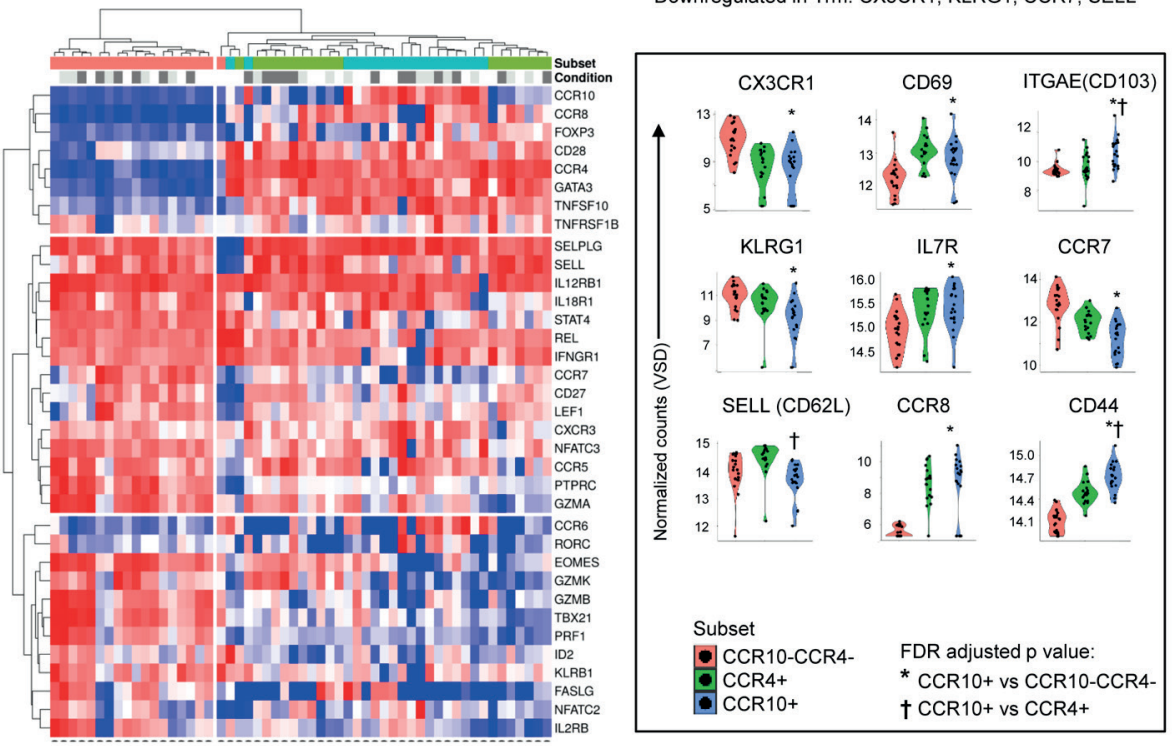

Fig 4. CCR10+ CD8+ T cells express GATA3, FOXP3 and tissue-resident memory profile

(A) Three different subsets of CD8 T cells were flow-sorted based on the presence/absence of CCR10 and CCR4 (full gating strategy Fig S5). (B) Principal components analysis based on pre-selection of 5268 differentially expressed genes (DEGs) between any of the cell subsets (nominal p-value $<0.05$ ). (C) Heatmap based on gene list from literature deemed critical for CD8+ T cells. (D) Violin plots of main transcriptional features attributed to tissue-resident memory $T$ cells ( $T r m$ ): text above the figure indicates whether these genes are prototypically up/down- regulated in Trm. Expression in the CCR10+ subset was compared to CCR10-CCR4- and to CCR4+. 
As expected, the dominant factor determining the overall transcriptomic profile are the cell subsets rather than patient/healthy status (depicted as "condition" in Fig 4C). Exploratory analysis on the CCR10+ CD8+ subset in PsA found 536 DEGs unique to PSA compared to Pso/HC (nominal P value $<0.05$ ), including upregulation of RORC, MYD88, and IFNAR1 (Fig S10). In summary, CCR10+ CD8+ T cells are characterized by high expression of GATA3, FOXP3 and core transcripts defining tissue-resident memory cells.

\section{CCR10+ CD8+ T cells have Tc2/Tc22 cytokine profile with net regulatory function}

In line with the transcriptomic profile, CCR10+ CD8+ T cells produced significantly more IL-17A and IL-22 compared to bulk CD8+ T cells upon ex-vivo re-stimulation. The production of IL-4, IL-13 and IL-10 was also enriched in CCR10+ CD8+ T cells. In contrast, CCR10+CD8+ T cells had reduced overall capacity to produce IFN-gamma and lacked markers (Granzyme B, Perforin) associated with cytotoxic capacity of CD8+ T cells (Fig5A-F) (Fig S11).

CCR10+ CD8+ T cells co-expressed Foxp3, and the CCR10+ CD8+ T cells frequency in PBMC was strongly correlated with the frequency of CD8+ T cells that expressed a "regulatory" phenotype (CD25+Foxp3+ and CD25+CD127-) (Fig 6A-B)(Fig S11). Considering that CCR10+CD8+ T cells had a pleiotropic cytokine-producing profile (Tc2/22-like), we next determined if CCR10+ CD8+ T cells have an overall immune regulatory function. To this end, we performed immunosuppression assays, using sorted CD8+ T cell subsets (selected based on the markers CCR10 and CCR4) and co-culturing them with autologous, cell-trace-violet labelled T cells (Fig $6 \mathbf{C}$ ). As compared to co-culture with bulk CD8+ T cells, the co-culture with CCR10+ CD8+ $T$ cells significantly reduced the proliferation of both CD4 T cells and CD8+ T cells (Fig 6D).

In summary, our functional assays confirms the transcriptome profile data as presented in Fig 4, indicating CCR10+ CD8+ T cells are TC2/22-like cells with overall regulatory function. 
A

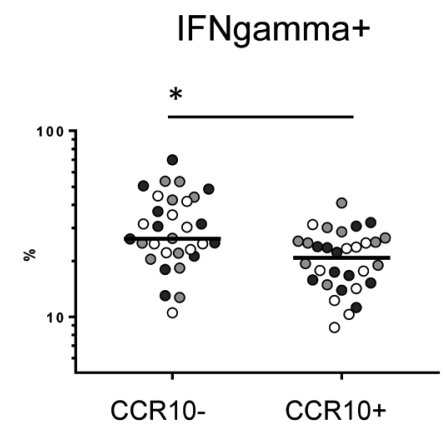

C

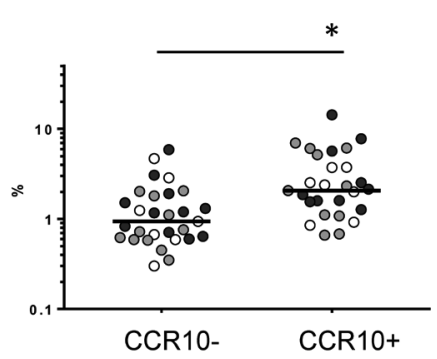

E

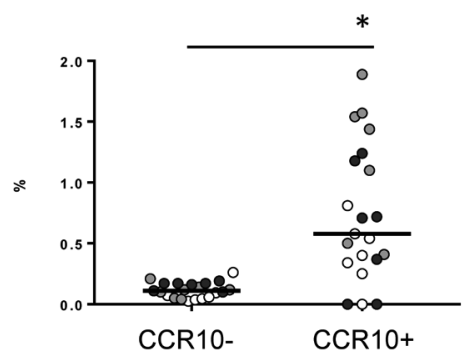

B

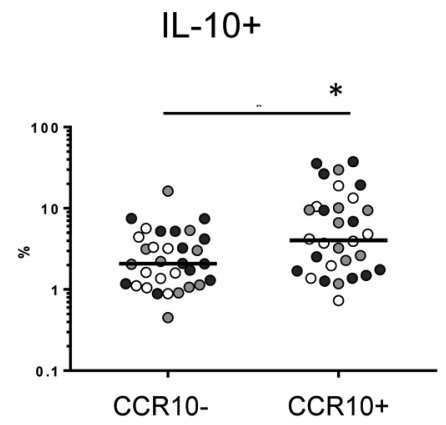

D IL-13+

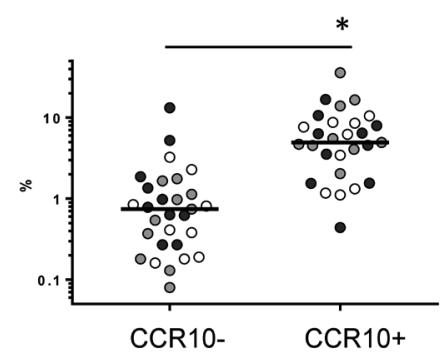

F $\quad$ IL-22+

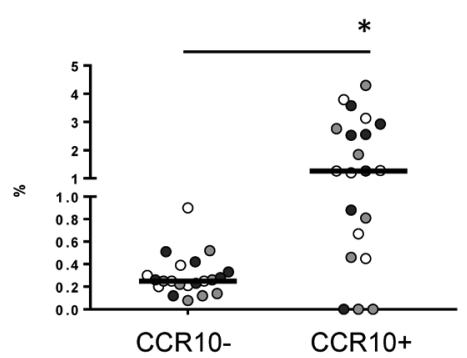

Fig 5. CCR10+ CD8+ T cells have Tc2/Tc22-like cytokine profile

(A-F) PBMC were re-stimulated with PMA/ionomycin and the frequency of intra-cellular cytokine production was compared between CD8+ T cells based on CCR10 expression. White circles indicate HC, grey circles indicate Pso, black circles indicate PsA. Graphs depict median. Asterisk indicates $p<0.05$. 
CD8+ CD45RO+ T cells

A

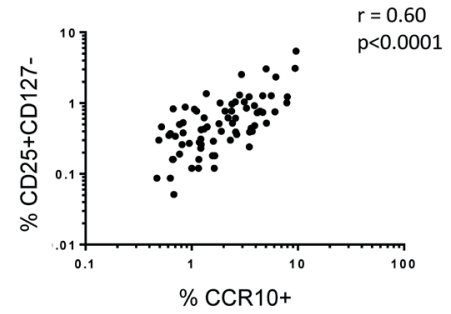

CD8+ CD45RO+ T cells

B

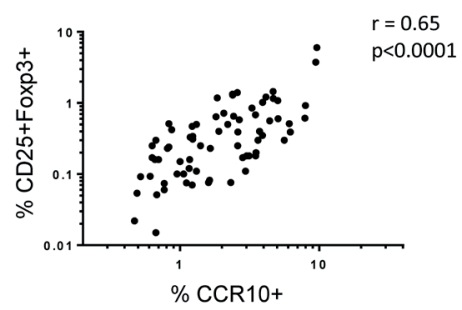

C

PBMC stimulated with anti-CD3 and co-cultured with autologous "regulatory-type" cells.

\section{"regulatory-type"}

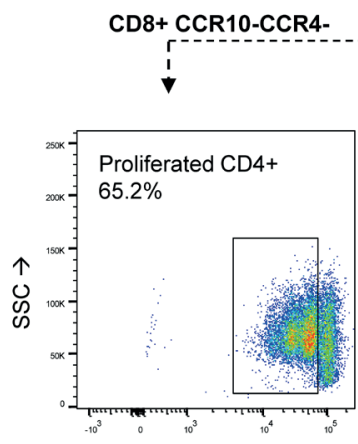

CT-violet $\rightarrow$

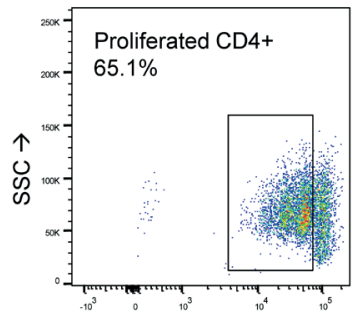

CT-violet $\rightarrow$
CD8 + CCR10+
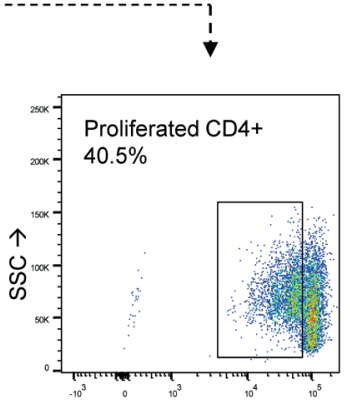

CT-violet $\rightarrow$
D

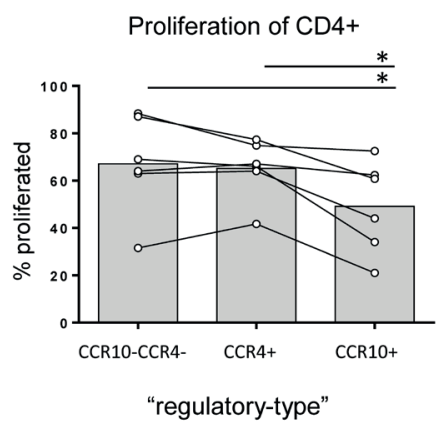

E

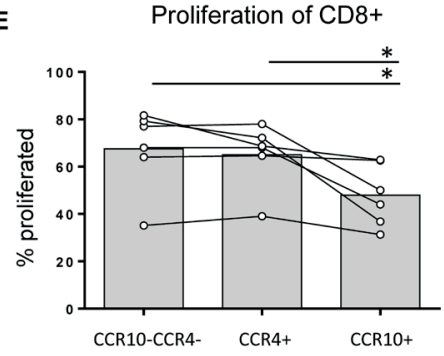

"regulatory-type"

Fig 6. CCR10+ CD8+ $T$ cells have regulatory properties

(A-B) In PBMC, the ex-vivo fraction of memory CD8+ T cells that express CCR10 was strongly correlated to the ex-vivo fraction of CD8+ T cells that express CD25+CD127- and CD25+Foxp3+. Circles indicate pooled data from HC and patients depicted in Table S1. (C) Example of suppression assay. Fresh PBMC $(n=5, H C)$ were incubated with CellTrace ${ }^{\mathrm{TM}}$ Violet and co-cultured with different CD8+ T cell subsets (flow sorting gating strategy Fig S5). (D-E) The suppressive effect on CD4+ and CD8+ T cell proliferation was determined at day four. Graphs depict median. Asterisk indicates $p<0.05$. 


\section{Discussion}

In this study we discovered and investigated in detail, an increase of CCR10+ CD8+ T cells in peripheral blood of patients with PSA as compared to Pso. CCR10+ CD8+ T cells are effector memory cells with Tc2/22-like cytokine profile and regulatory function. This CD8+ T cell subset was further endowed with transcriptomic profile comparable to tissue-resident memory $T$ cells and their origin could be tracked back to the skin, but not the joint.

To our knowledge this is the broadest immunophenotyping study thus far, as performed in a PASI-matched cohort of PSA and Pso patients free from immunomodulatory drugs. Overall, the results underscore the role of memory CD8+ T cells in the pathogenesis of PSA, in line with previous immunophenotyping studies and genetic association studies(1,11,25-27). Specifically, we have identified a novel role for CCR10+ CD8+ T cells which may be important in the pathogenesis of PsA.

The fact that skin-homing markers have previously been described on $T$ cells in synovial fluid/tissue(27-29) and that therapeutics blocking specific integrins can induce arthritis(30,31), has raised the question whether (skin-tropic) T cells could be re-directed into the joint. However, our results do not indicate CCR10+ CD8+ T cells are derived from the synovial fluid, nor do they appear to preferentially migrate to this site. Instead, we found typical co-expression pattern with skin-tropic markers CLA and CCR4, in line with previous work that indicates CCR10 to guide trafficking of $T$ cells towards the skin(22,32). The CCR10+ CD8+ T cells are most likely found in the epidermis(33). Notably, we found that both lesional and nonlesional skin harbours CCR10+CD8+ T cells. There was a trend for fewer regulatory CCR10+ CD8+ T cells in lesional skin, which may contribute to the pathology. We suspect that CCR10+ CD8+ T cells from non-lesional skin contributes to the fraction detected in PBMCs, for a number of reasons including (i) the Pso and PSA had similar PASI score, (ii) there was no relationship to PASI and (iii) even in healthy individuals these cells are detected in circulation(34). Larger studies will be needed to compare the quantity of CCR10+ CD8+ T cells in skin from Pso and PsA, particularly to examine if there are subtle changes between these groups with respect to both lesional and non-lesional sites.

Phenotypically, CCR10+ CD8 + T cells were mostly effector memory as based on classic nomenclature, but we also detected a strong transcriptional overlap to skin-derived, tissue-resident memory T cells(35). In human skin, CD8+ T cells can be classified as those that pass through the tissue and those that remain in the tissue, termed tissue-resident memory (Trm) cells. Trm can be divided into CD69+CD103- or CD69+CD103+, the latter more prevalent in epidermis with 
potent effector functions(36,37). Our transcriptomic analysis of CCR10+ CD8+ T cells revealed striking resemblance to Trm: high expression of IL7R (CD127), CD69, ITGAE (CD103) and low expression of KLRG1 and CX3CR1(32,36,37). Furthermore, transcriptomic analysis revealed high expression of $C C R 8$, which has recently been linked to a skin-resident CD8+ T cell population(32). Recent work in a murine model tracked memory precursor CD8+ T cell clones from circulation and found that high expression of CCR10 was found in CD8+ T cells committed to a skin Trm fate(38). Strictly speaking the CCR10+ CD8+ T cells we characterized in PBMCs should not be termed Trm, since Trm are per definition non-circulating(39). Nonetheless, this dichotomy may be too simplistic, at least when it comes to CD4 T cells: CLA+CD4 Trm can exit skin, re-enter the circulation and occupy distant skin sites(34). Our data fit well into this recent concept of circulating Trm and specifically adds strength to the case for circulating human $\operatorname{CD} 8+\operatorname{Trm}(40,41)$.

In terms of function, CCR10 has classically been attributed to T helper 2 and T helper 22 subsets of CD4 T cells(42). The CCR10+ CD8+ T cells we investigated indeed depicted Tc2/22-like function, but are distinct from Tc17 recently described in PsA synovial fluid(27), including the fact that the Tc17 expressed high levels of granzymes. CCR10 has also been described as common marker on regulatory CD4 T cells(43). Loss of CCR10 in murine models results in loss of regulatory $T$ cells in the skin, which enhances IL-17A and TNFa production at the cost of IL10 production $(24,44,45)$. This indicates CCR10 is important for the regulatory function of tissue resident T cells in the murine non-inflamed skin $(24,45)$. Overall, the transcriptomic profile and functional assays performed in our study indicate these cells could have an important regulatory function in human skin.

There are different proposed mechanisms by which CD8+ T cells can induce a suppressive function(46). The exact mechanisms by which these cells induced suppressive function lies beyond the scope of our current study. The potential role for immune-checkpoints including DNAM1-TIGIT axis warrant further investigation, since we noted phenotypic disturbances in the PsA group. The balance of regulation versus inflammation could also be secondary to environmental cues that skew cell plasticity and cytokine profile, as shown for CD4 T cells and innate lymphoid cells $(47,48)$. Consistent with the latter concept is that we noted RORC and IFNAR1, amongst other genes, to differentiate the CCR10+ CD8+ T cells from PsA versus Pso/HC. While RORC is critical to the pathogenic effects of innate immune cells in spondyloarthritis(49), our transcriptomic analysis within the PsA group remains exploratory and deserves confirmation in a larger group of patients.

The potential novel role of CD8+ Trm in PsA, we argue should be placed in context of the more established role for CD8+ Trm in psoriasis(33,40,50,51). Clinically unaffected skin from psoriasis patients has perturbations in keratinocytes and 
Trm populations, suggested to poise the skin for an excessive inflammatory response(51). Also, after psoriasis lesions have clinically resolved there is longterm persistence of epidermal CD8+ Trm with IL-17A producing capacities $(40,50)$. Of further interest is that the effector function CD8+ Trm in non-lesional is related to disease duration(33).

The study methods have certain limitations, which includes the fact that our study was cross-sectional in design and that we did not include non-spondyloarthritis patients (e.g. rheumatoid arthritis or gout). Although we adhered to a standardized protocol for flow cytometry(14), viability staining was only performed in the functional and transcriptomic analysis. Also, upon identification of CCR10 as discriminatory marker between PsA and psoriasis within CD8+CD45RO+ T cells, for further experiments we made the practical choice to exclude CD45RO as marker in the gating which may have resulted in the inclusion of some naïve CD8+ T cells. Importantly, it will be required for these results to be validated in an independent cohort of patients, regardless of any subtle modifications to the flow-cytometric gating strategy employed.

One of the open questions that remains unanswered at this point is if the CCR10+ CD8+ T cells are themselves pathogenic in PsA (e.g. contributing to production of soluble factors affecting distant musculoskeletal sites) or instead should be seen as the flag of disturbed cutaneous homeostasis that is principally driven by other cells, such as keratinocytes or other stromal cells.

Taken together, our study indicates PsA is marked by alterations in circulating, skin-derived, regulatory CD8+ Trm. These data support that events occurring in the skin might drive development of arthritis in patients with psoriasis.

Acknowledgements: We would like to thank the patients for participating in the study, the clinical study team (Anne Karien Marijnissen, Anneloes van Loo, Karin Schrijvers and Joke Nijdeken) and the flow core facility of the Center for Translational Immunology. We would like to thank Jonas Kuipers for his scientific input. 


\section{References}

1. Veale DJ, Fearon U. The pathogenesis of psoriatic arthritis. Lancet (London, England). 2018;391(10136):2273-84.

2. Ritchlin CT, Colbert RA, Gladman DD. Psoriatic Arthritis. N EngIJ Med. 2017;376(10):957-70.

3. Sakkas LI, Bogdanos DP. Are psoriasis and psoriatic arthritis the same disease? The IL-23/IL17 axis data. Autoimmun Rev. 2017 Jan;16(1):10-5.

4. Boehncke W-H. Psoriasis and Psoriatic Arthritis: Flip Sides of the Coin? Acta Derm Venereol. 2016 May;96(4):436-41.

5. McGonagle D, Aydin SZ, Gül A, Mahr A, Direskeneli H. 'MHC-I-opathy'-unified concept for spondyloarthritis and Behçet disease. Nat Rev Rheumatol. 2015;11(12):731-40.

6. Sherlock JP, Joyce-Shaikh B, Turner SP, Chao C-C, Sathe M, Grein J, et al. IL-23 induces spondyloarthropathy by acting on ROR-yt+ CD3+CD4-CD8- entheseal resident $\mathrm{T}$ cells. Nat Med. 2012 Jul;18(7):1069-76.

7. Reinhardt A, Prinz I. Whodunit? The Contribution of Interleukin (IL)-17/IL-22-Producing y $\delta T$ Cells, a $\mathrm{\beta}$ T Cells, and Innate Lymphoid Cells to the Pathogenesis of Spondyloarthritis. Front Immunol. 2018;9:885.

8. Abji F, Pollock RA, Liang K, Chandran V, Gladman DD. Brief Report: CXCL10 Is a Possible Biomarker for the Development of Psoriatic Arthritis Among Patients With Psoriasis. Arthritis Rheumatol (Hoboken, NJ). 2016;68(12):2911-6.

9. Abji F, Pollock RA, Liang K, Chandran V, Gladman DD. Th17 gene expression in psoriatic arthritis synovial fluid and peripheral blood compared to osteoarthritis and cutaneous psoriasis. Clin Exp Rheumatol. 36(3):486-9.

10. Pollock RA, Abji F, Liang K, Chandran V, Pellett FJ, Virtanen C, et al. Gene expression differences between psoriasis patients with and without inflammatory arthritis. J Invest Dermatol. 2015 Feb;135(2):620-3.

11. Benham H, Norris P, Goodall J, Wechalekar MD, Fitzgerald O, Szentpetery A, et al. Th17 and Th22 cells in psoriatic arthritis and psoriasis. Arthritis Res Ther. 2013 Jan;15(5):R136.

12. Taylor W, Gladman D, Helliwell P, Marchesoni A, Mease P, Mielants H. Classification criteria for psoriatic arthritis: development of new criteria from a large international study. Arthritis Rheum. 2006 Aug;54(8):2665-73.

13. Rudwaleit M, van der Heijde D, Landewe R, Listing J, Akkoc N, Brandt J, et al. The development of Assessment of SpondyloArthritis international Society classification criteria for axial spondyloarthritis (part II): validation and final selection. Ann Rheum Dis. 2009 Jun 1;68(6):777-83.

14. Verhagen FH, Hiddingh S, Rijken R, Pandit A, Leijten E, Olde Nordkamp M, et al. HighDimensional Profiling Reveals Heterogeneity of the Th17 Subset and Its Association With Systemic Immunomodulatory Treatment in Non-infectious Uveitis. Front Immunol. 2018 Oct 31;9:2519.

15. Wehrens EJ, Mijnheer G, Duurland CL, Klein M, Meerding J, van Loosdregt J, et al. Functional human regulatory $T$ cells fail to control autoimmune inflammation due to PKB/c-akt hyperactivation in effector cells. Blood. 2011 Sep 29;118(13):3538-48. 


\section{CHAPTER 6}

16. Cunningham F, Amode MR, Barrell D, Beal K, Billis K, Brent S, et al. Ensembl 2015. Nucleic Acids Res. 2015 Jan 28;43(D1):D662-9.

17. Dobin A, Davis CA, Schlesinger F, Drenkow J, Zaleski C, Jha S, et al. STAR: ultrafast universal RNA-seq aligner. Bioinformatics. 2013 Jan;29(1):15-21.

18. Anders S, Pyl PT, Huber W. HTSeq--a Python framework to work with high-throughput sequencing data. Bioinformatics. 2015 Jan 15;31(2):166-9.

19. Love MI, Huber W, Anders S. Moderated estimation of fold change and dispersion for RNAseq data with DESeq2. Genome Biol. 2014 Dec 5;15(12):550.

20. Xia J, Wishart DS. Using MetaboAnalyst 3.0 for Comprehensive Metabolomics Data Analysis. Curr Protoc Bioinforma. 2016 Sep;55(1):14.10.1-14.10.91.

21. Oliveros JC. Venny. An interactive tool for comparing lists with Venn's diagrams.

22. Sigmundsdottir H, Pan J, Debes GF, Alt C, Habtezion A, Soler D, et al. DCs metabolize sunlightinduced vitamin D3 to "program" T cell attraction to the epidermal chemokine CCL27. Nat Immunol. 2007 Mar;8(3):285-93.

23. Homey B, Alenius $H$, Müller A, Soto H, Bowman EP, Yuan W, et al. CCL27-CCR10 interactions regulate T cell-mediated skin inflammation. Nat Med. 2002 Feb;8(2):157-65.

24. Xia M, Hu S, Fu Y, Jin W, Yi Q, Matsui Y, et al. CCR10 regulates balanced maintenance and function of resident regulatory and effector $T$ cells to promote immune homeostasis in the skin. J Allergy Clin Immunol. 2014 Sep;134(3):634-644.e10.

25. Menon B, Gullick NJ, Walter GJ, Rajasekhar M, Garrood T, Evans HG, et al. Interleukin$17+C D 8+T$ cells are enriched in the joints of patients with psoriatic arthritis and correlate with disease activity and joint damage progression. Arthritis Rheumatol (Hoboken, NJ). 2014 May;66(5):1272-81.

26. Bowes J, Budu-Aggrey A, Huffmeier U, Uebe S, Steel K, Hebert HL, et al. Dense genotyping of immune-related susceptibility loci reveals new insights into the genetics of psoriatic arthritis. Nat Commun. 2015 Feb 5;6:6046.

27. Steel KJA, Srenathan U, Ridley M, Durham LE, Wu S-Y, Ryan SE, et al. Polyfunctional, Proinflammatory, Tissue-Resident Memory Phenotype and Function of Synovial Interleukin-17A+CD8+ T Cells in Psoriatic Arthritis. Arthritis Rheumatol (Hoboken, NJ). 2020 Mar;72(3):435-47.

28. Jones SM, Dixey J, Hall ND, McHugh NJ. Expression of the cutaneous lymphocyte antigen and its counter-receptor E-selectin in the skin and joints of patients with psoriatic arthritis. $\mathrm{Br} J$ Rheumatol. 1997 Jul;36(7):748-57.

29. Pitzalis C, Cauli A, Pipitone N, Smith C, Barker J, Marchesoni A, et al. Cutaneous lymphocyte antigen-positive $\mathrm{T}$ lymphocytes preferentially migrate to the skin but not to the joint in psoriatic arthritis. Arthritis Rheum. 1996 Jan;39(1):137-45.

30. Viguier M, Richette $\mathrm{P}$, Aubin F, Beylot-Barry M, Lahfa M, Bedane C, et al. Onset of psoriatic arthritis in patients treated with efalizumab for moderate to severe psoriasis. Arthritis Rheum. 2008 Jun;58(6):1796-802.

31. Dubash S, Marianayagam T, Tinazzi I, Al-Araimi T, Pagnoux C, Weizman A V, et al. Emergence of severe spondyloarthropathy-related entheseal pathology following successful vedolizumab therapy for inflammatory bowel disease. Rheumatology (Oxford). 2019;58(6):963-8. 
32. McCully ML, Ladell K, Andrews R, Jones RE, Miners KL, Roger L, et al. CCR8 Expression Defines Tissue-Resident Memory T Cells in Human Skin. J Immunol. 2018 Feb 2;ji1701377.

33. Vo S, Watanabe R, Koguchi-Yoshioka H, Matsumura Y, Ishitsuka Y, Nakamura Y, et al. CD8 resident memory $T$ cells with interleukin 17A-producing potential are accumulated in disease-naiive nonlesional sites of psoriasis possibly in correlation with disease duration. $\mathrm{Br} \mathrm{J}$ Dermatol. 2019 Aug 29;181(2):410-2.

34. Klicznik MM, Morawski PA, Höllbacher B, Varkhande SR, Motley SJ, Kuri-Cervantes L, et al. Human CD4 + CD103 + cutaneous resident memory T cells are found in the circulation of healthy individuals. Sci Immunol. 2019 Jul 5;4(37):eaav8995.

35. Romero P, Zippelius A, Kurth I, Pittet MJ, Touvrey C, lancu EM, et al. Four functionally distinct populations of human effector-memory CD8+ T lymphocytes. J Immunol. 2007 Apr 1;178(7):4112-9.

36. Watanabe R, Gehad A, Yang C, Scott LL, Teague JE, Schlapbach C, et al. Human skin is protected by four functionally and phenotypically discrete populations of resident and recirculating memory T cells. Sci Transl Med. 2015 Mar 18;7(279):279ra39.

37. Mackay LK, Rahimpour A, Ma JZ, Collins N, Stock AT, Hafon M-L, et al. The developmental pathway for CD103(+)CD8+ tissue-resident memory T cells of skin. Nat Immunol. 2013 Dec;14(12):1294-301.

38. Kok L, Dijkgraaf FE, Urbanus J, Bresser K, Vredevoogd DW, Cardoso RF, et al. A committed tissue-resident memory $T$ cell precursor within the circulating CD8+ effector T cell pool. J Exp Med. 2020 Oct 5;217(10).

39. Morris SE, Farber DL, Yates AJ. Tissue-Resident Memory T Cells in Mice and Humans: Towards a Quantitative Ecology. J Immunol. 2019 Nov 15;203(10):2561-9.

40. Cheuk S, Schlums H, Gallais Sérézal I, Martini E, Chiang SC, Marquardt N, et al. CD49a Expression Defines Tissue-Resident CD8 + T Cells Poised for Cytotoxic Function in Human Skin. Immunity. 2017 Feb;46(2):287-300.

41. Khalil S, Bardawil T, Kurban M, Abbas O. Tissue-resident memory T cells in the skin. Inflamm Res. 2020 Mar 27;69(3):245-54.

42. Trifari S, Kaplan CD, Tran EH, Crellin NK, Spits $H$. Identification of a human helper $T$ cell population that has abundant production of interleukin 22 and is distinct from $T(H)-17, T(H) 1$ and T(H)2 cells. Nat Immunol. 2009 Aug; 10(8):864-71.

43. Eksteen B, Miles A, Curbishley SM, Tselepis C, Grant AJ, Walker LSK, et al. Epithelial Inflammation Is Associated with CCL28 Production and the Recruitment of Regulatory T Cells Expressing CCR10. J Immunol. 2006 Jul 1;177(1):593-603.

44. Fu Y, Yang J, Xiong N. Cutting Edge: Skin CCR10+ CD8+ T Cells Support Resident Regulatory T Cells through the B7.2/Receptor Axis To Regulate Local Immune Homeostasis and Response. J Immunol. 2016;196(12):4859-64.

45. Li C, Xu M, Coyne J, Wang W-B, Devila M, Wang Y, et al. Psoriasis-associated impairment of CCL27/CCR10-derived regulation leads to IL-17A/IL-22-producing skin T cell over-activation. J Allergy Clin Immunol. 2020 Jun; 
46. Xu Z, Ho S, Chang C-C, Zhang Q-Y, Vasilescu E-R, Vlad G, et al. Molecular and Cellular Characterization of Human CD8 T Suppressor Cells. Front Immunol. 2016 Nov 30;7.

47. Bernink JH, Ohne Y, Teunissen MBM, Wang J, Wu J, Krabbendam L, et al. c-Kit-positive ILC2s exhibit an ILC3-like signature that may contribute to IL-17-mediated pathologies. Nat Immunol. 2019;20(8):992-1003.

48. Zielinski CE, Mele F, Aschenbrenner D, Jarrossay D, Ronchi F, Gattorno M, et al. Pathogeninduced human TH17 cells produce IFN-y or IL-10 and are regulated by IL-1 $\beta$. Nature. 2012 Apr 26;484(7395):514-8.

49. Venken K, Jacques P, Mortier C, Labadia ME, Decruy T, Coudenys J, et al. RORyt inhibition selectively targets IL-17 producing iNKT and $\gamma \delta-T$ cells enriched in Spondyloarthritis patients. Nat Commun. 2019;10(1):9.

50. Cheuk S, Wikén M, Blomqvist L, Nylén S, Talme T, Ståhle M, et al. Epidermal Th22 and Tc17 Cells Form a Localized Disease Memory in Clinically Healed Psoriasis. J Immunol. 2014 Apr 1;192(7):3111-20.

51. Gallais Sérézal I, Hoffer E, Ignatov B, Martini E, Zitti B, Ehrström M, et al. A skewed pool of resident $T$ cells triggers psoriasis-associated tissue responses in never-lesional skin from patients with psoriasis. J Allergy Clin Immunol. 2019 Apr;143(4):1444-54. 


\section{Supplementary Material}

\section{Supplementary Tables}

- Table S1 - Demographic and clinical characteristics

- Table S2 - List of antibodies

\section{Supplementary Figures}

- Figure S1 - Gating strategy used for dendritic cell panel

- Figure S2 - Gating strategy used for T cell surface panel

- Figure S3 - Gating strategy used for T regulatory panel

- Figure S4 - Gating strategy used for T cell intracellular panel

- Figure S5 - Gating strategy used for flow cytometry cell sorting

- Figure S6 - CCR10+ CD8+ T cells are effector memory, enriched for CCR6

- Figure S7 - Gating strategy used for skin and SFMC

- Figure S8 - CD103 expression by CD8+ T cells per location

- Figure $\mathbf{S 9}$ - Transcriptome of CCR10+ CD8+ T cells

- Figure $\mathbf{S 1 0}$ - Transcriptome of CCR10+ CD8+ T cells specifically in PsA

- Figure S11 - CCR10+ CD8+ T cells lack granzyme B or perforin and co-express Foxp3 
CHAPTER 6

Table S1

\begin{tabular}{|c|c|c|c|c|}
\hline & $\mathrm{HC}$ & Pso & PsA & AxSpA \\
\hline Subjects & 23 & 21 & 21 & 16 \\
\hline Age (years) & $43 \pm 2.3$ & $42 \pm 2.5$ & $42 \pm 2.4$ & $49 \pm 3.4$ \\
\hline Female & $9(39 \%)$ & $10(48 \%)$ & $7(33 \%)$ & $5(31 \%)$ \\
\hline \multicolumn{5}{|l|}{ Disease dur. (years) } \\
\hline Pso & . & $11(3.9-23)$ & $14(8.6-22)$ & . \\
\hline PsA & . & . & $0.9(0.25-5.7)$ & . \\
\hline $\mathrm{AxSpA}$ & . & . & . & $11(7.9-23)$ \\
\hline \multicolumn{5}{|l|}{ Disease activity } \\
\hline PASI & . & $6.5 \pm 1.4$ & $5.9 \pm 1.2$ & . \\
\hline SJC (66) & . & . & $6.5 \pm 1.2$ & 0 \\
\hline TJC (68) & . & . & $6.2 \pm 1.0$ & 0 \\
\hline Dactylitis present & . & . & $2(9.5 \%)$ & 0 \\
\hline Enthesitis count & . & . & $0.55 \pm 0.18$ & $0.13 \pm 0.13$ \\
\hline $\mathrm{CRP}(\mathrm{mg} / \mathrm{L})$ & . & $1.6(0.93-5.5)$ & $3.1(2-7)$ & $3.9(1.6-6.2)$ \\
\hline $\operatorname{ESR}(\mathrm{mm} / \mathrm{h})$ & . & $5(2-7.8)$ & $10(3-16)$ & $5(2.3-12)$ \\
\hline BASDAI & . & . & . & $3.5 \pm 0.59$ \\
\hline \multicolumn{5}{|l|}{ Treatment } \\
\hline current b/cDMARD & . & $0(0 \%)$ & $0(0 \%)^{*}$ & $0(0 \%)$ \\
\hline past b/cDMARD & . & $2(9.5 \%)$ & $4(19 \%)$ & $2(13 \%)$ \\
\hline
\end{tabular}

\section{Table S1. Demographic and clinical characteristics}

The demographic and clinical characteristics of the patients that were used for the four PBMC immunophenotyping panels. Data presented as mean \pm SEM, median (IQR), or number (\%). There were no significant differences between Pso and PSA for the variables listed.

Abbreviations: Psoriasis (Pso); Psoriatic Arthritis(PsA); Axial spondyloarthritis (AxSpA); psoriasis area severity index (PASI); swollen joint count (SJC); tender joint count (TJC); C-reactive protein (CRP); erythrocyte sedimentation rate (ESR); Bath Ankylosing Spondylitis Disease Activity Index (BASDAI); disease-modifying antirheumatic drug (DMARD). *One patient with PsA received treatment with oral prednisone. 
Table S2. List of antibodies used

\begin{tabular}{|c|c|c|c|}
\hline marker & label & clone & company \\
\hline 7-AAD & PercP & & $\mathrm{BD}$ \\
\hline CCR10 & PE & 314305 & R\&D Systems \\
\hline CCR10 & APC & 314305 & R\&D Systems \\
\hline CCR4 & FITC & 205410 & $R \& D$ \\
\hline CCR4 & PE & $1 \mathrm{G} 1$ & BD \\
\hline CCR4 & BV605 & $1 \mathrm{G} 1$ & BD Horizon \\
\hline CCR6 & PE & $11 \mathrm{~A} 9$ & BD \\
\hline CD103 & $\mathrm{PE}$ & Ber-АСТ8 & BD \\
\hline CD11C & PE-CF594 & B-ly6 & BD Horizon \\
\hline CD123 & FITC & $7 G 3$ & $\mathrm{BD}$ \\
\hline CD127 & AF647 & HCD127 & Sony Biotechnology \\
\hline CD127 & BV421 & HIL-7R-M21 & BD Horizon \\
\hline CD14 & FITC & TÜK4 & Miltenyi \\
\hline CD14 & eFluor780 & 61D3 & eBioscience \\
\hline CD141 & BV711 & $1 \mathrm{~A} 4$ & BD Horizon \\
\hline CD16 & BV510 & $3 \mathrm{G} 8$ & BD Horizon \\
\hline CD161 & BV510 & DX12 & BD Horizon \\
\hline CD169 & PE & $7-239$ & eBioscience \\
\hline CD19 & AF700 & HIB19 & eBioscience \\
\hline CD1c & APC & AD5-8E7 & Miltenyi \\
\hline CD25 & PE-Cy7 & M-A251 & $\mathrm{BD}$ \\
\hline CD25 & $\mathrm{PE}$ & $2 \mathrm{~A} 3$ & $\mathrm{BD}$ \\
\hline CD27 & eFluor780 & L128 & eBioscience \\
\hline CD27 & BV510 & L128 & BD Horizon \\
\hline CD3 & PE-Cy7 & UCHT1 & Biolegend \\
\hline CD3 & AF700 & UCHT1 & BioLegend \\
\hline CD303 & PE-Cy7 & $201 \mathrm{~A}$ & BioLegend \\
\hline CD4 & APC-eF780 & RPA-T4 & eBioscience \\
\hline CD4 & BV711 & RPA-T4 & Sony Biotechnology \\
\hline CD4 & PerCP-CY5.5 & SK3 & $\mathrm{BD}$ \\
\hline CD4 & eFluor780 & RPA-T4 & eBioscience \\
\hline CD45 & PacOrange & HI30 & Life Technologies \\
\hline CD45 & BV711 & HI30 & Biolegend \\
\hline CD45RO & BV711 & UCHL1 & BioLegend \\
\hline CD56 & AF700 & B159 & $\mathrm{BD}$ \\
\hline
\end{tabular}


CHAPTER 6

\begin{tabular}{llll}
\hline marker & label & clone & company \\
\hline CD56 & PE-CF594 & B159 & BD \\
CD8 & V500 & RPA-T8 & BD \\
CD8 & PE-Cy7 & SK1 & BD \\
CLA & PB & HECA-452 & Biolegend \\
CXCR3 & FITC & G025H7 & BioLegend \\
CXCR5 & BV421 & RF8B2 & BD \\
DNAM1 & APC & 11A8 & Biolegend \\
FoxP3 & APC & PCH101 & eBioscience \\
gammadelta-TCR & FITC & IMMU510 & Beckman Coulter \\
granzyme B & FITC & GB11 & BD \\
HLA-DR & BV421 & L243 & BioLegend \\
IFN-y & PerCP-CY5.5 & 4S.B3 & eBioscience \\
IL-10 & PE & JES3-19F1 & BD \\
IL-10 & APC & JES3-19F1 & BD \\
IL-13 & FITC & B-P6 & Serotec \\
IL-17A & FITC & EBio64DEC17 & eBioscience \\
IL-21 & PE & 3A3-N2.1 & BD \\
IL-22 & PE-Cy7 & 22URTI & eBioscience \\
IL-22 & APC & IL22JOP & eBioscience \\
IL-4 & BV711 & MP4-25D2 & BD \\
IL-9 & PE-CF594 & MH9A3 & BD Horizon \\
Integrin b7 & BV421 & FIB504 & BD \\
Perforin & PE & 27-35 & BD \\
TIGIT & BV421 & MBSA43 & eBioscience \\
TNF-a & EFluor780 & MAb11 & BD Horizon \\
Viability & eF506 & & ebioscience \\
Viability & & ebioscience \\
\hline & & \\
\hline
\end{tabular}


Figure S1. Gating strategy used for dendritic cell panel

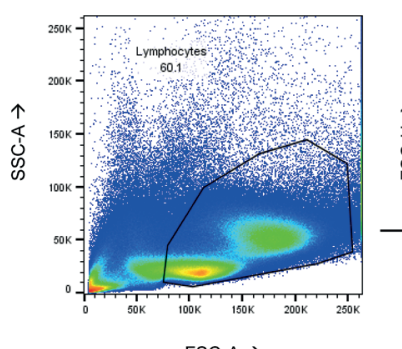

FSC-A $\rightarrow$
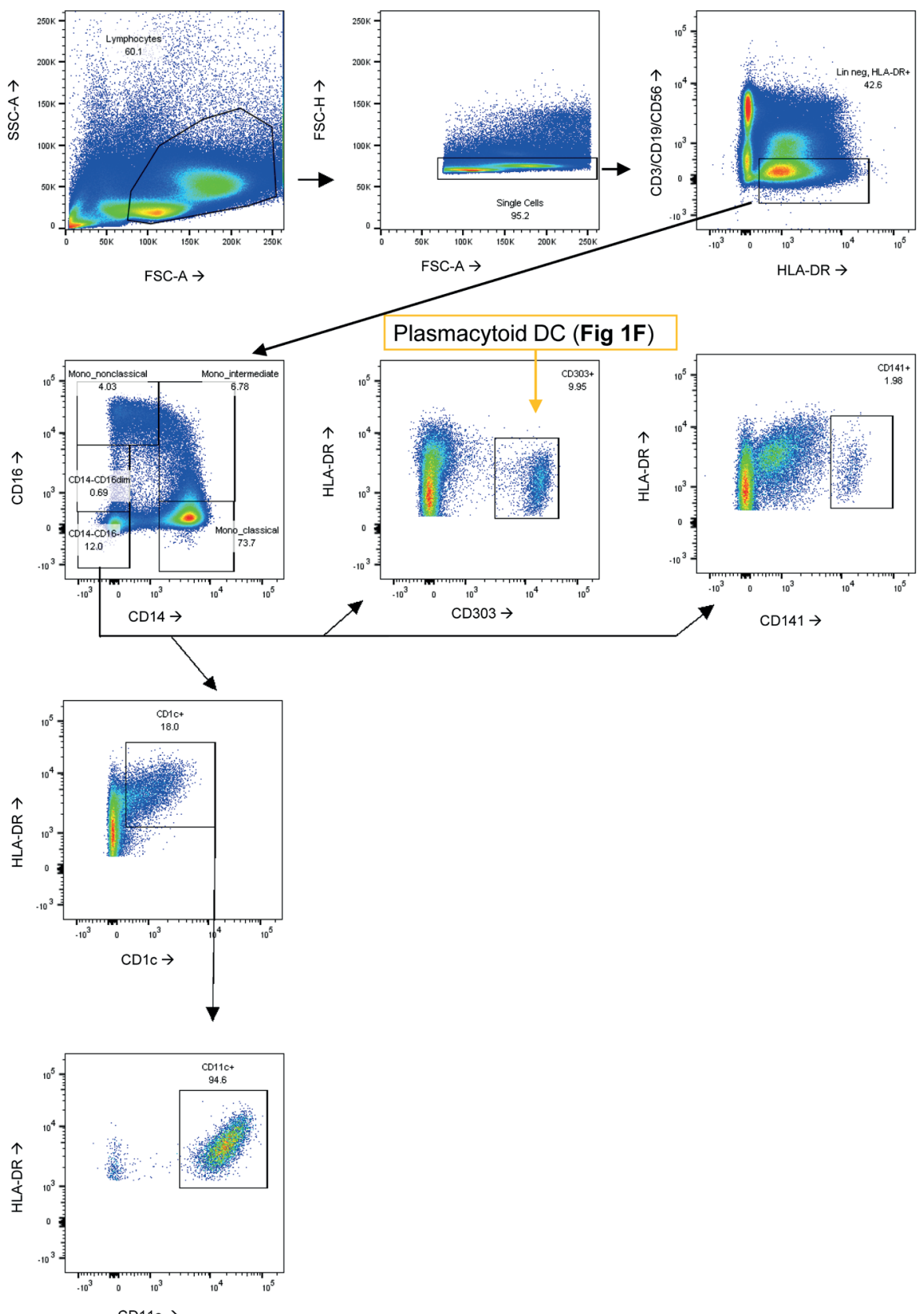

$\mathrm{CD} 11 \mathrm{c} \rightarrow$ 
Figure S2. Gating strategy used for T cell surface panel
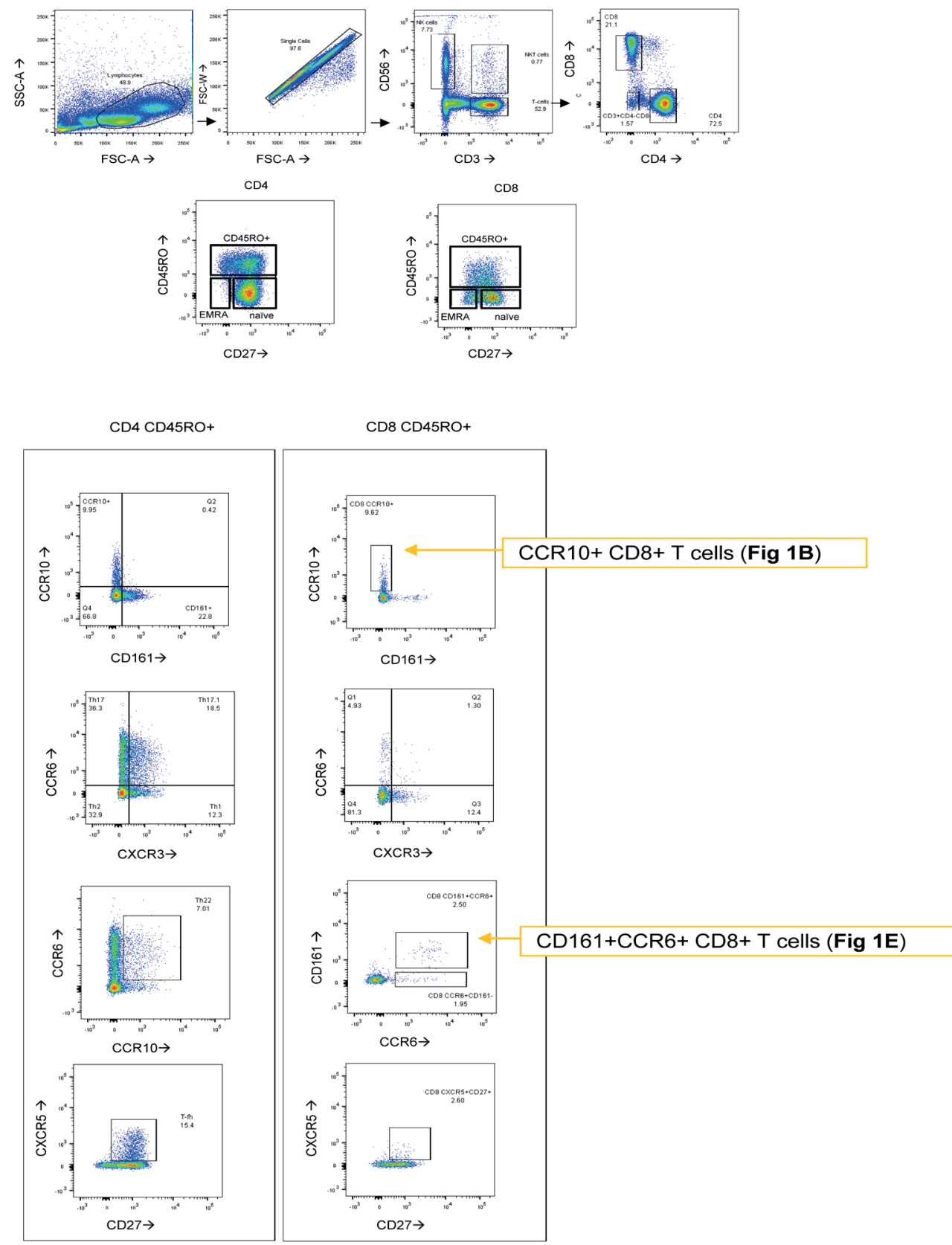
Figure S3. Gating strategy used for T regulatory panel

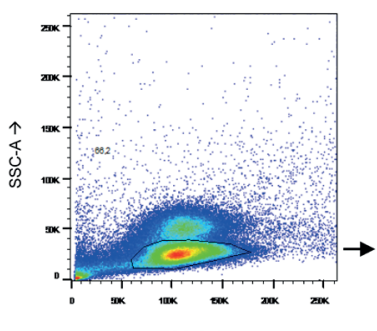

FSC-A $\rightarrow$

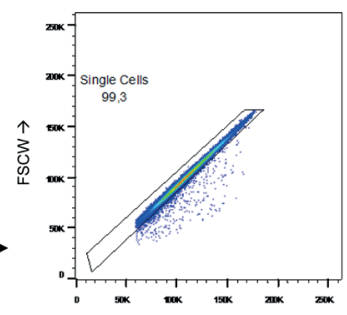

FSC-A $\rightarrow$
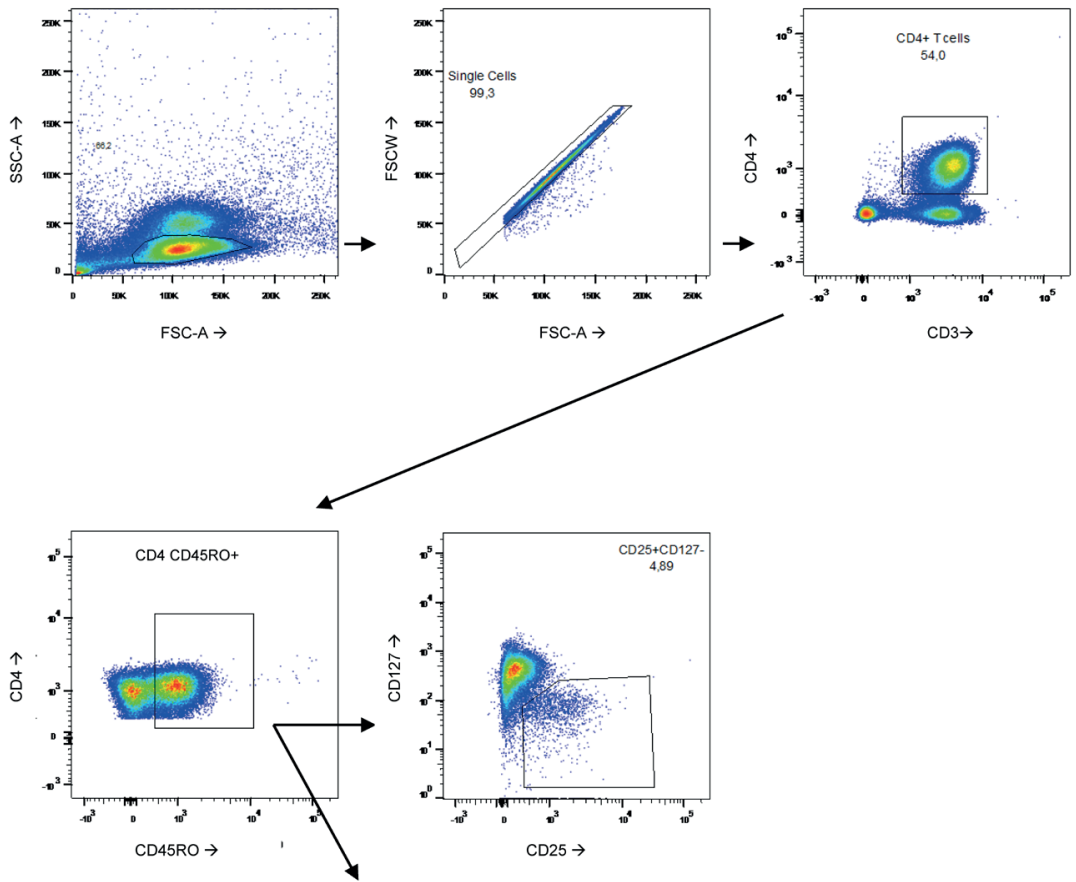

Regulatory CD4+ T cells (Fig 1D)

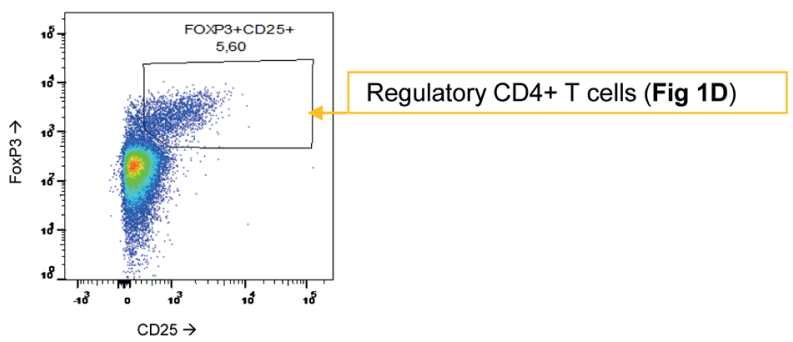


Figure S4. Gating strategy used for T cell intracellular panel
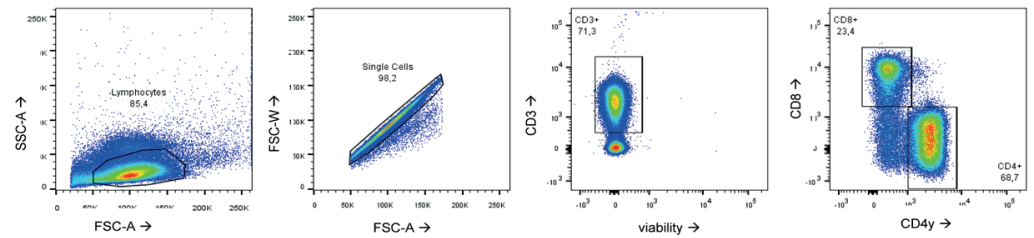

CD4 $\mathrm{T}$ cells
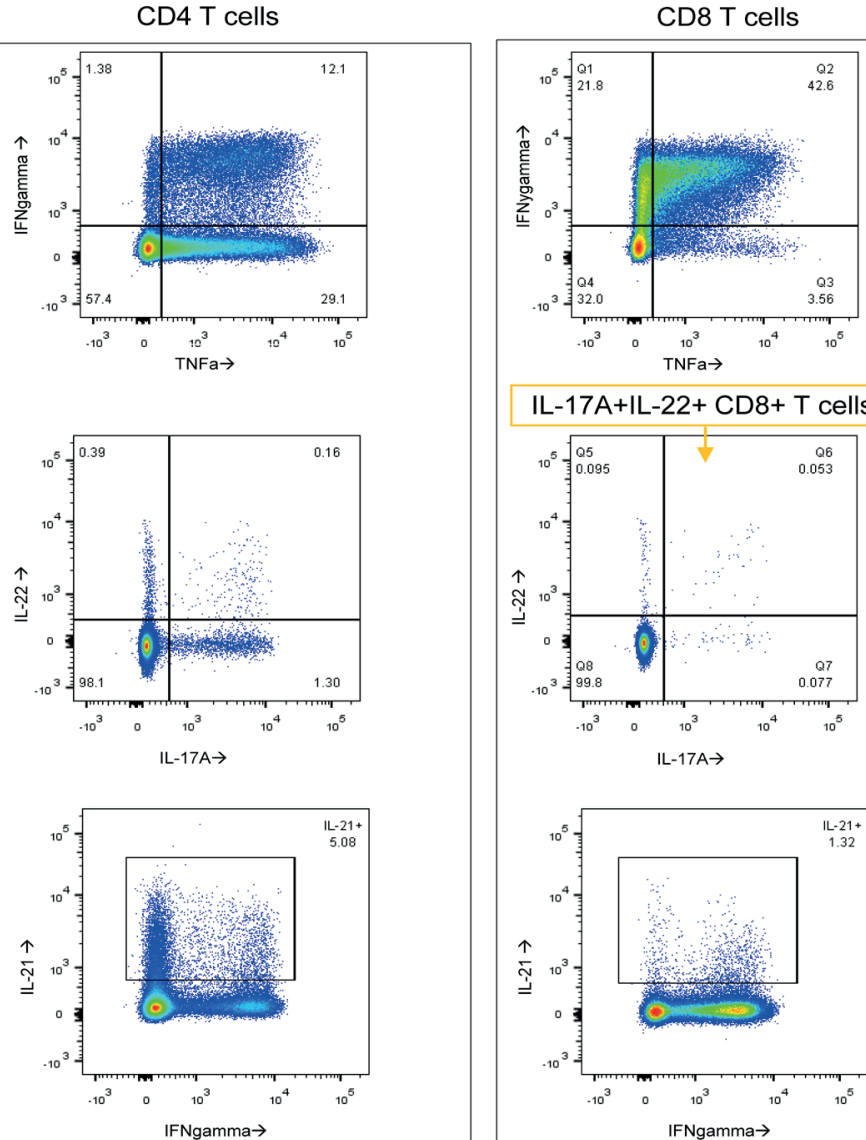

\section{IL-17A+IL-22+ CD8+ T cells (Fig 1C)}
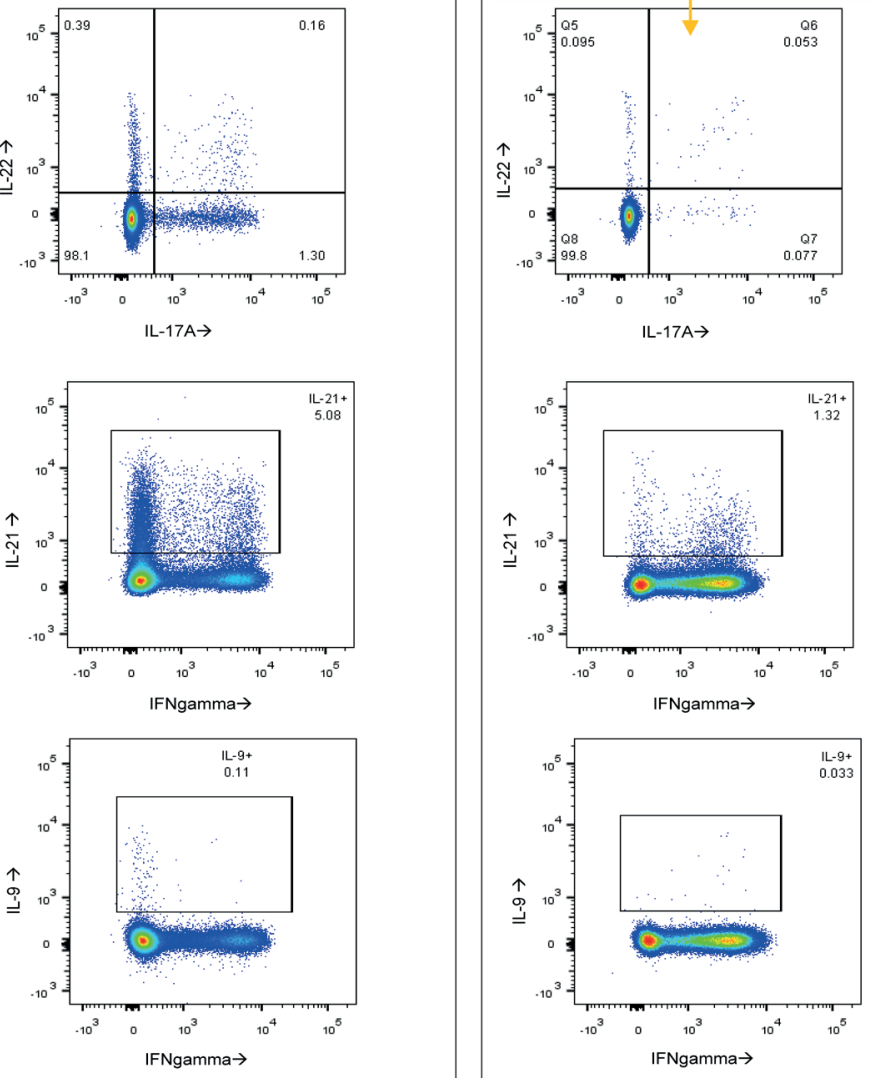
Figure S5. Gating strategy used for flow cytometry cell sorting

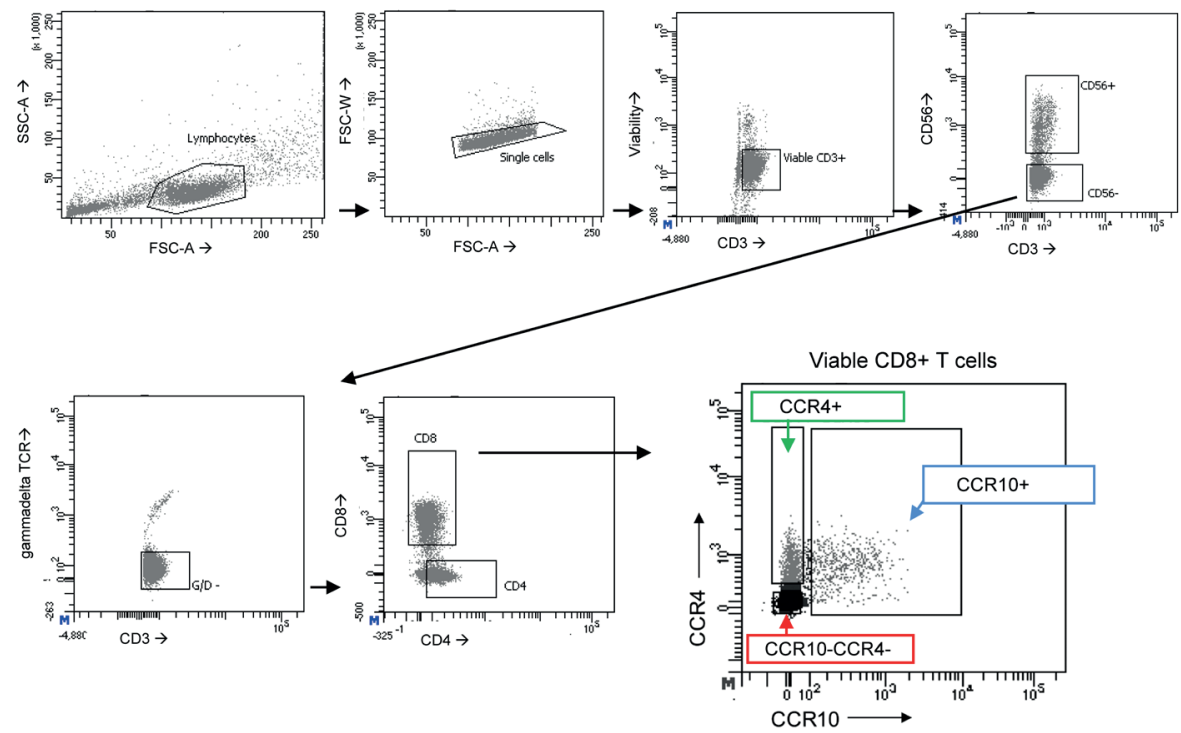

Figure S5. Gating strategy used for flow cytometry cell sorting. Gating strategy used for flow cytometric cell sorting of CD8+ T cell subsets which were used for transcriptomic analysis and suppression assays. PBMCs were sorted based on scatter, single, viable, CD3+CD56- $\delta$-CD8+ and then three subsets were sorted: CCR10+ (regardless of CCR4 co-expression), CCR4+ (strictly CCR10-) and CCR10-CCR4-. 
Figure S6. CCR10+ CD8 T cells are typically central memory and effector memory

A

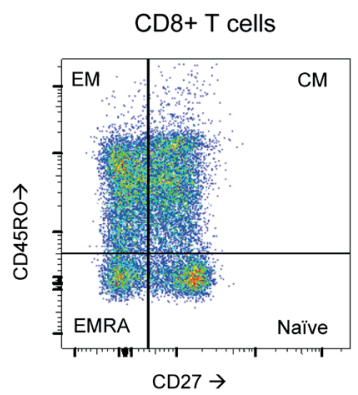

C

\section{CCR10+}

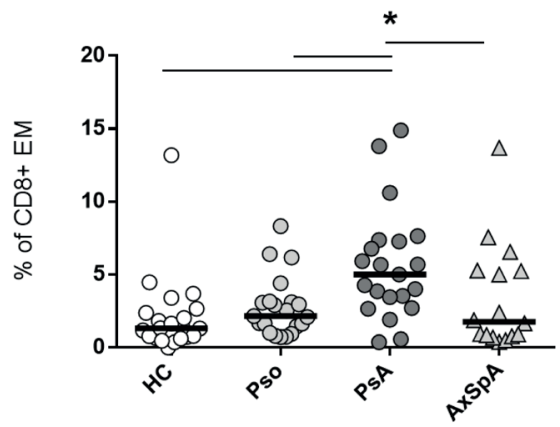

B

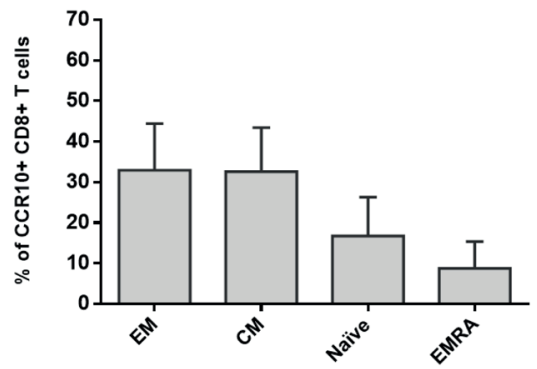

D

\section{CCR10+}

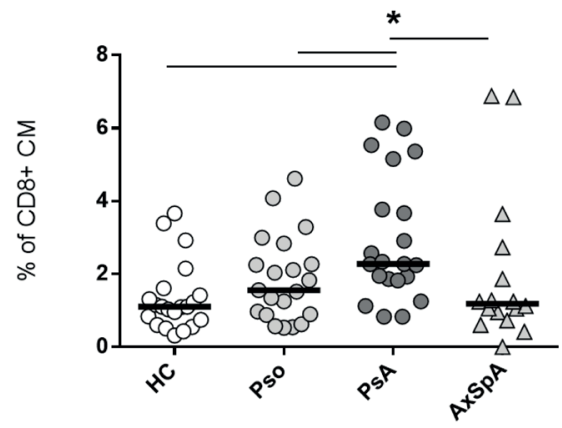

Figure S6. CCR10+ CD8 T cells are typically central memory or effector memory

(A) Following the gating strategy of Fig S2 to identify CD8+ T cells, the CD45RO vs CD27 quadrant gate is applied to distinguish CD8+ T cell subsets: naïve, central memory (CM), effector memory (EM), or effector memory cells re-expressing CD45RA (EMRA).

(B) Based on all CCR10 + CD8+ T cells, the majority of these cells are subsequently classified as EM or CM, although some naïve and EMRA are included when directly gating on CCR10+ CD8 T cells. Graphs depict median+IQR, from pooled data from patients and $\mathrm{HC}$.

(C-D) Figure 1B displayed the CCR10 expression as \% of all memory CD8 T cells (regardless of CD27). These plots show CCR10 expression specifically as \% of EM CD8+ T cell subsets (C) or as \% of CM CD8+ T cells (D). *The frequency of CCR10 expression was significantly higher in PSA as compared to HC, Pso and AS ( $p<0.05)$. 
Figure S7. Gating strategy used for skin and SFMC
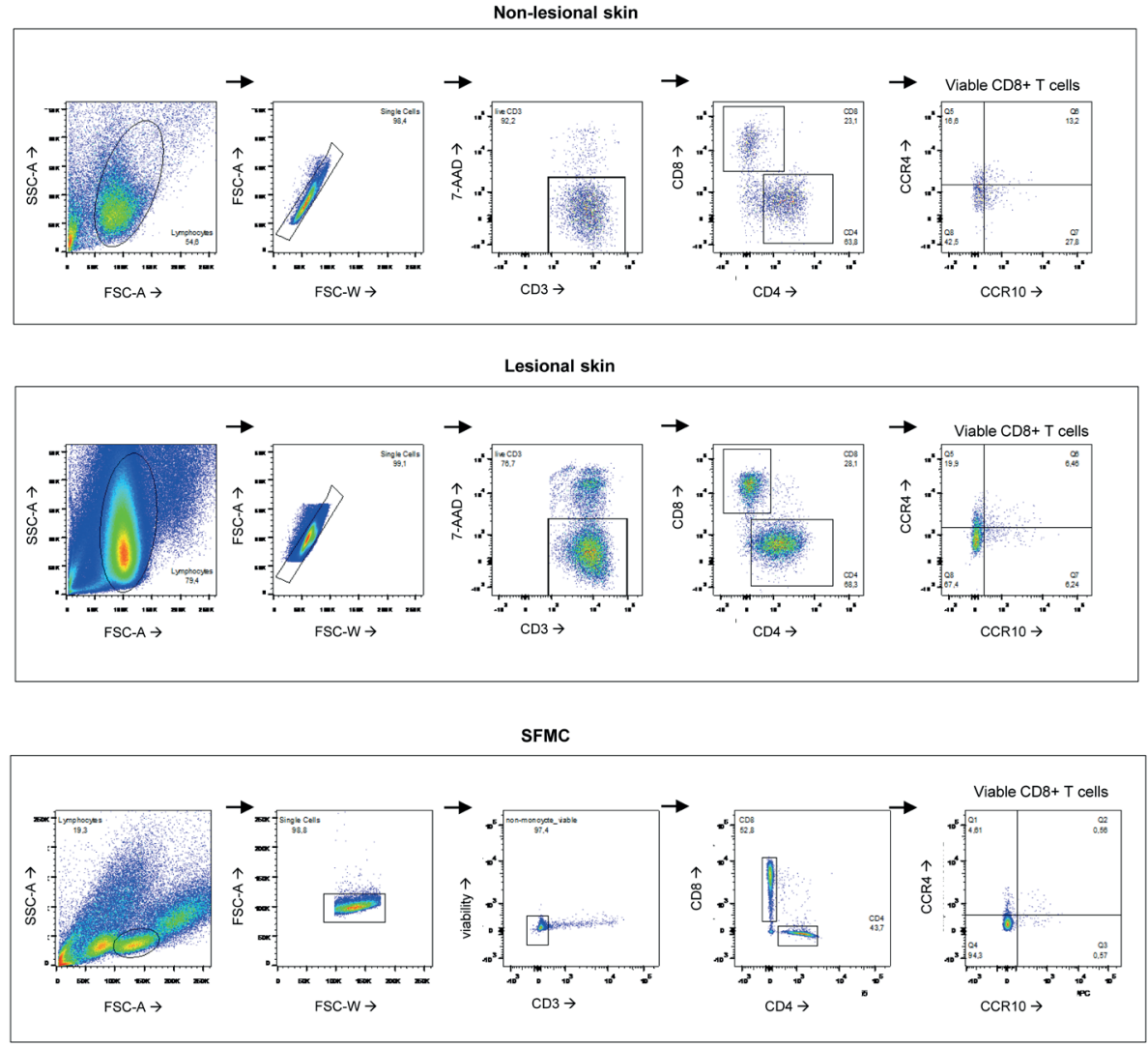

Control (CCR10 FMO)

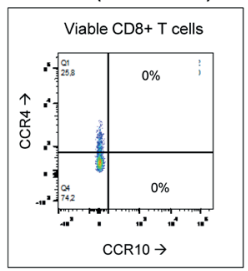

Figure S7. Gating strategy used for skin and SFMC

SFMC = synovial fluid mononuclear cells

$\mathrm{FMO}=$ fluorescence minus one for CCR10, as control for manual gating 
Figure S8. CD103 expression by CD8 T cells per location

\section{CD8+ T cells co-expressing CD103}

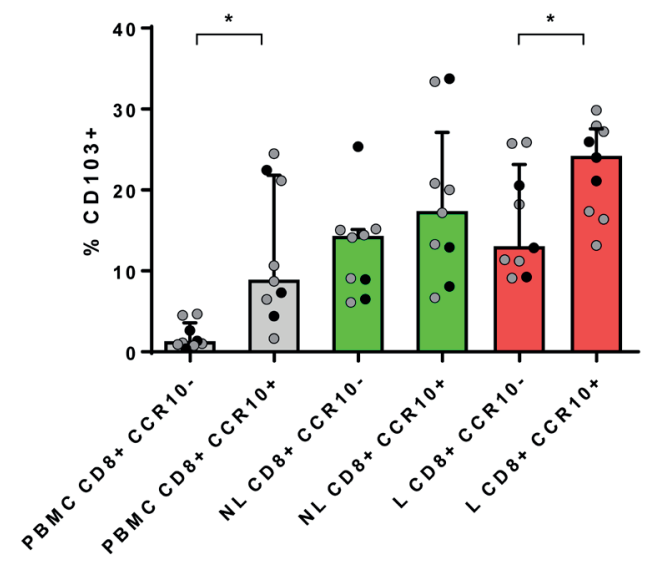

Figure S8. CD103 expression by CD8+ T cells per location

CD103 expression in viable CD8+ T cells (either CCR10+ or CCR10-) per compartment.

SFMC = synovial fluid mononuclear cells

$\mathrm{PBMC}=$ peripheral blood mononuclear cells

$\mathrm{NL}=$ non-lesional skin

$\mathrm{L}=$ lesional skin 
Figure S9. Transcriptome of CCR10+ CD8 T cells

\section{Selection of genes, from top 100 DEGs comparing CCR10+ vs CCR10-CCR4-}

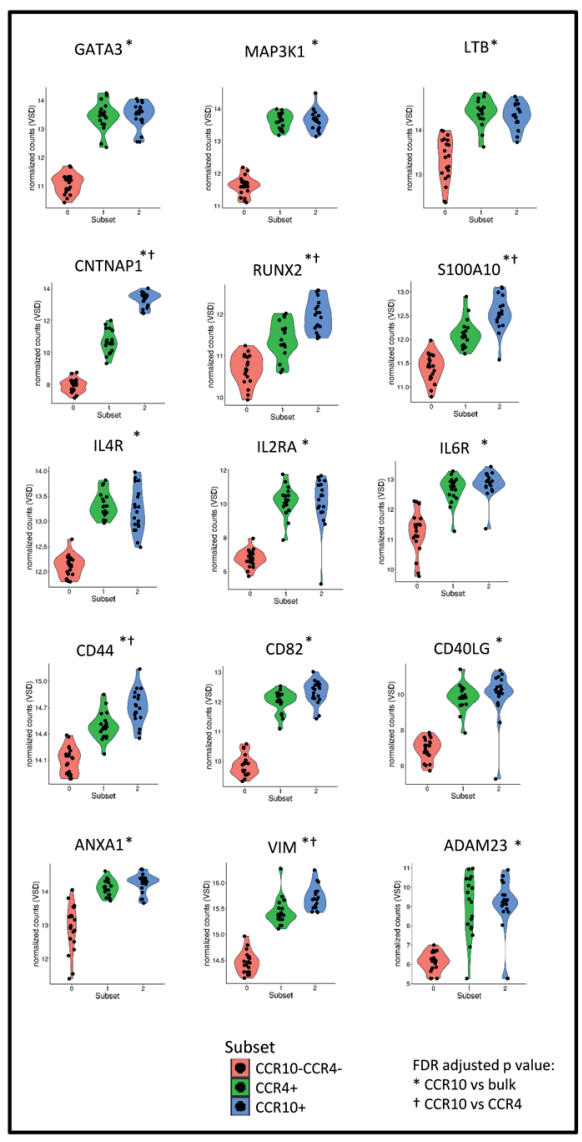

DEGs comparing CD8+ T cell subsets

(FDR adjusted p-value $<0.05$ )

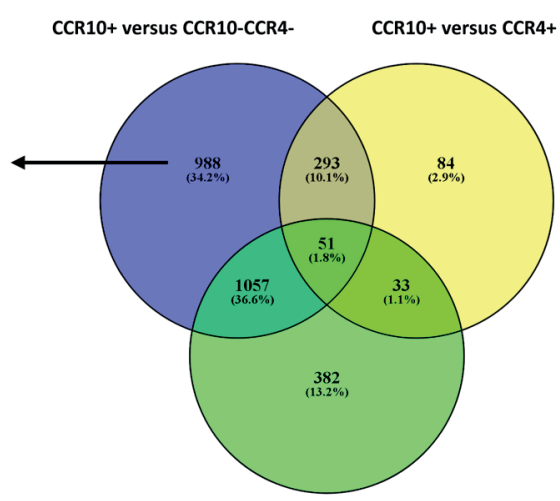

CCR4+ versus CCR10-CCR4-

Figure S9. Transcriptome of CCR10+ CD8+ cells

Top left: Violin plots of selection of the top 100 DEGs between CCR10+ and bulk.

Top right: Venn diagram of DEGs comparing the different subsets (selected on HGCN-coding only). 
Figure S10. Transcriptome of CCR10+ CD8 T cells specifically in PSA
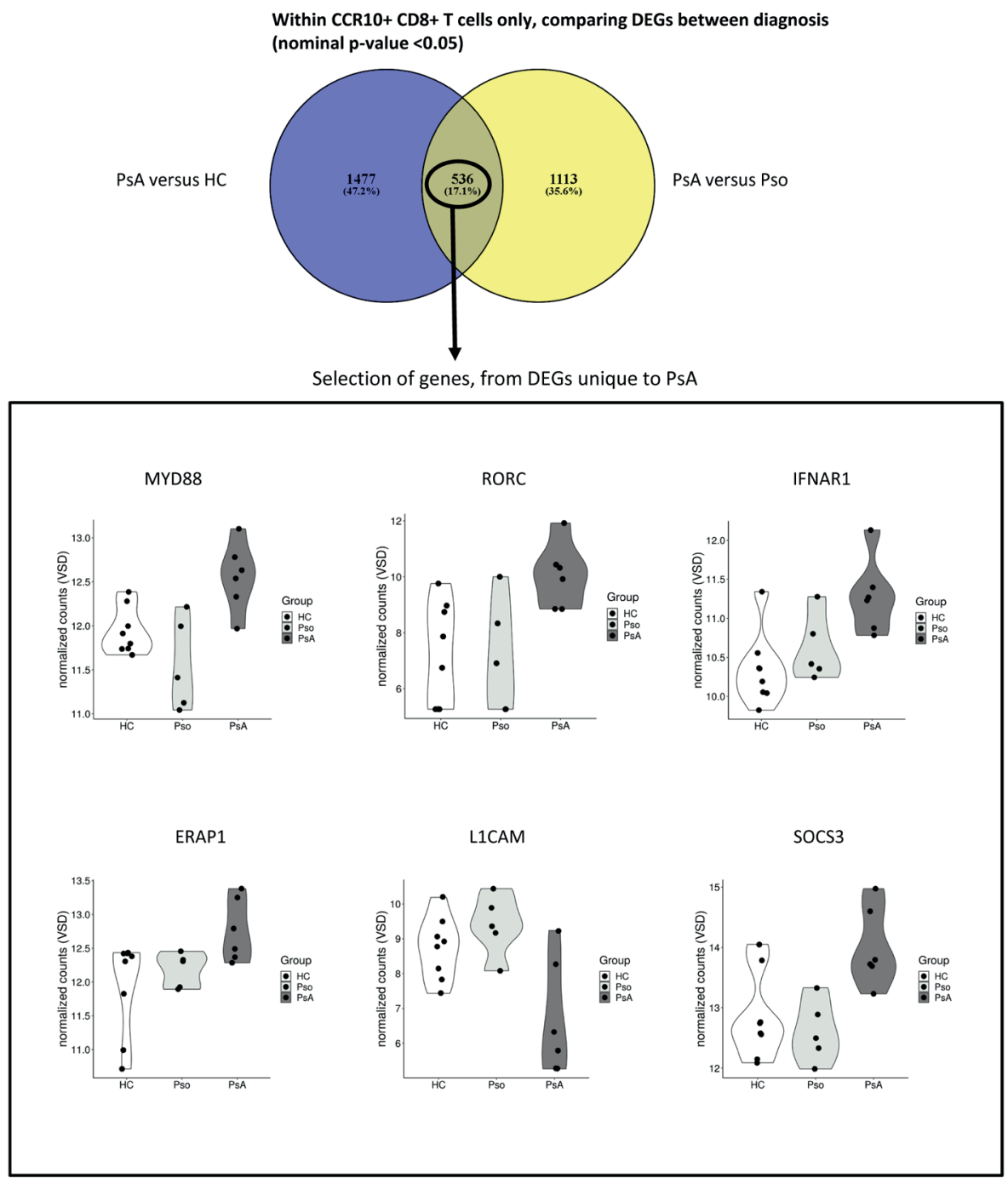

Figure S10. Transcriptome of CCR10+ CD8+ cells specifically in PSA

Top: Venn diagram of DEGs (nominal $p<0.05$ ) comparing the CCR10+ subset between patient groups (selected on HGCN-coding only).

Bottom: Selection of violin plots depicting DEGs (nominal $p<0.05$ ) unique to the CCR10+ CD8 + T cells subset in PsA vs Pso/HC. 
Figure S11. CCR10+ CD8+ T cells lack granzyme B or perforin and co-express FoxP3

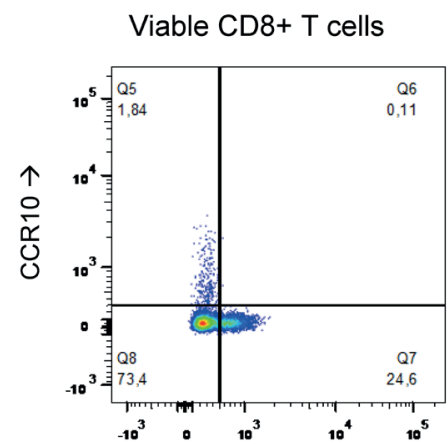

Granzyme B $\rightarrow$
Viable CD8+ T cells

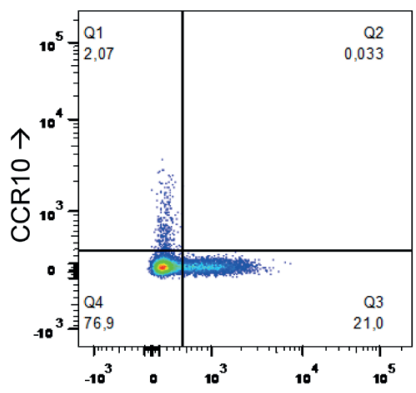

Perforin $\rightarrow$

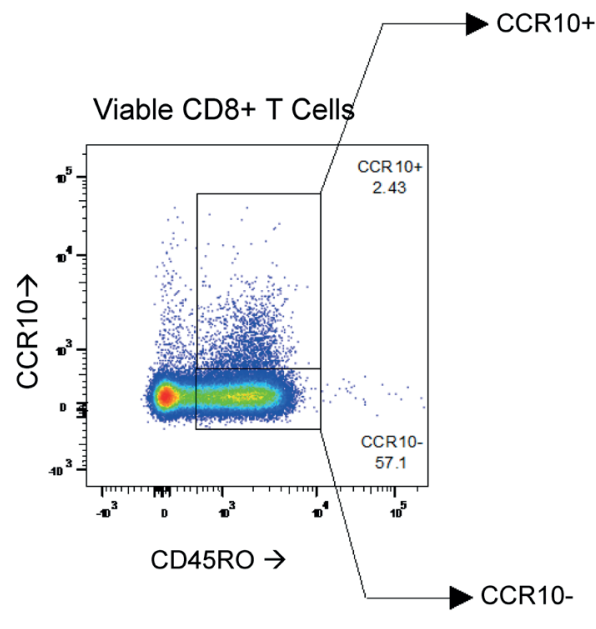

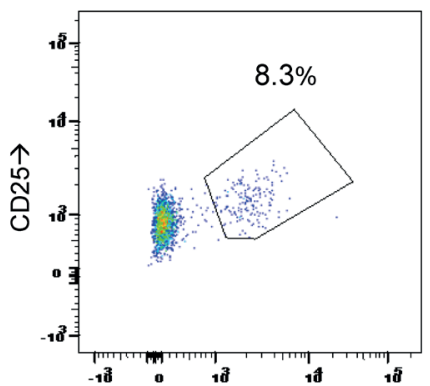

Foxp3 $\rightarrow$

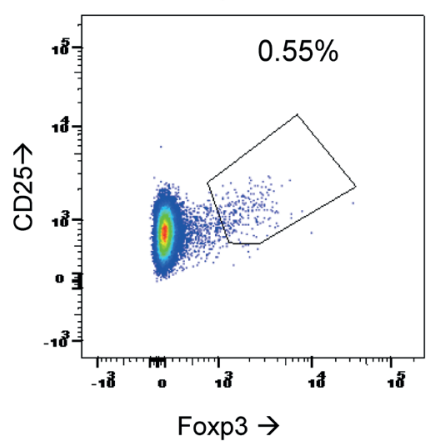

Figure S11. CCR10+ CD8+ T cells lack granzyme B or perforin and co-express FoxP3

Top: Upon re-stimulation, CCR10+ CD8+ T cells lacked granzyme B or perforin co-expression. Representative sample of $n=3$.

Bottom: The Foxp3 ${ }^{\text {high }} \mathrm{CD} 25+$ phenotype is enriched in CCR10+ CD8+ T cells compared to CCR10- CD8+ T cells. Representative sample of $n=10$. 


$$
7
$$




\section{CHAPTER 7}

\section{Broad proteomic screen reveals shared serum proteomic signature in patients with psoriatic arthritis and psoriasis without arthritis}

Authors:

Leijten EFA ${ }^{1,2} *$, Tao $W^{1,2} *$, Pouw $\mathrm{JN}^{1,2}$, van Kempen $\mathrm{TS}^{1,2}$, Olde Nordkamp $\mathrm{M}^{1,2}$, Balak DMW ${ }^{4}$, Tekstra J1', Muñoz-Elías EJ ${ }^{5}$, DePrimo SE ${ }^{5}$, Drylewicz J2 , Pandit $A^{1,2}$, Boes $\mathrm{M}^{2,3}$, Radstake TRDJ ${ }^{1,2}$

*authors contributed equally

Affiliations:

1. Department of Rheumatology and Clinical Immunology, UMC Utrecht, Utrecht, Netherlands 2. Center for Translational Immunology, UMC Utrecht, Utrecht, Netherlands 3. Department of Pediatrics, UMC Utrecht, Utrecht, Netherlands 4. Department of Dermatology, UMC Utrecht, Utrecht, Netherlands 5. Immunology Biomarkers, Janssen Research \& Development LLC, San Diego, CA, USA

Published in:

Rheumatology (Oxford). 2020 Aug. Online ahead of print doi: 10.1093/rheumatology/keaa405 


\section{ABSTRACT}

Objective: To identify novel serum proteins involved in the pathogenesis of PsA as compared to healthy controls, psoriasis (Pso) and AS, and to explore which proteins best correlated to major clinical features of the disease.

Methods: A high-throughput serum biomarker platform (Olink) was used to assess the level of 951 unique proteins in serum of patients with PsA $(n=20), \operatorname{Pso}(n=18)$ and AS $(n=19)$ as well as healthy controls $(H C, n=20)$. Pso and PsA were matched for Psoriasis Area and Severity Index (PASI) and other clinical parameters.

Results: We found 68 differentially expressed proteins (DEPs) in PsA as compared to HC. Of those DEPs, 48 proteins (71\%) were also dysregulated in Pso and/or AS. Strikingly, there were no DEPs when comparing PsA to Pso directly. On the contrary, hierarchical cluster analysis and multidimensional scaling revealed that $\mathrm{HC}$ clustered distinctly from all patients, and that PsA and Pso grouped together. The number of swollen joints (SJC) had the strongest positive correlation to ICAM$1(r=0.81, p<0.001)$ and CCL18 $(r=0.76, p<0.001)$. PASI score was best correlated to PI3 $(r=0.54, p<0.001)$ and IL-17 receptor $A(r=-0.51, p<0.01)$. There were more proteins correlated to PASI score when analyzing Pso and PSA patients separately, as compared to analyzing Pso and PsA patients pooled together.

Conclusions: PsA and Pso patients share a serum proteomic signature, which supports the concept of a single psoriatic spectrum of disease. Future studies should target skin and synovial tissues to uncover differences in local factors driving arthritis development in Pso. 


\section{INTRODUCTION}

Psoriasis (Pso) is a common auto-immune disease causing excessive scaling, redness, and itching of skin at prototypical sites of the body. Approximately twenty percent of patients with Pso will at some point in their life develop psoriatic arthritis (PsA) (1). A clinical diagnosis of PsA is typically made in a patient with Pso or psoriatic nail disease with concomitant arthritis. PsA is clinically heterogeneous and other manifestations include those of the spondyloarthritis spectrum, such as enthesitis, dactylitis, and spondyloarthritis. Adding to this heterogeneity is that in approximately $15 \%$ of the cases of PsA, arthritis manifests prior to Pso (1). Both cutaneous and rheumatic manifestations of Pso negatively impact quality of life and should be treated appropriately (2).

Tremendous advances have been made in the treatment options available for Pso. The current and emerging therapeutics can almost completely reverse skin inflammation in a majority of patients, but their capacity at halting arthritis is less impressive (3). This discrepancy is well-illustrated by examining current gold standard of trial outcome measures: a 90\% percent improvement for Pso disease severity (Psoriasis Area and Severity Index, PASI90), compared with a 20\% improvement for arthritis severity (ACR20). Numerous factors could explain the trailing treatment response in arthritis, including drug bioavailability, the cellular target and cellular turnover at the target tissue, as well as (still unidentified) differences in tissue-specific drivers of pathogenesis (4-6).

It is unknown if the immunologic drivers in Pso patients versus PsA patients are different $(7,8)$. This raises the question whether these diseases are part of the same spectrum or distinct entities $(8,9)$. Pso is one of the strongest known clinical risk factors for the development of arthritis, thus providing a unique opportunity to better understand arthritis development and improve treatment. It has historically been difficult to identify early PsA in Pso patients in daily clinical practice and there are currently no serum diagnostic biomarkers used in care. This impedes clarification of the presence or absence of a window-of-opportunity for treating early PsA. To overcome these important open questions, Pso and PsA should be studied head-to-head to uncover potential differences in pathogenesis that could serve as therapeutic targets, as well as identify possible biomarkers to be used in early diagnosis.

Genetic studies reveal vast overlap between Pso and PsA, in which the few differences found were variants related to chromatin marks on a subset of $\mathrm{T}$ lymphocytes, CD8 T cells, and to variants in the IL-23 receptor $(10,11)$. In comparative studies from peripheral blood mononuclear cells, Pso patients with PsA have higher expression of genes associated with the interferon signature in their monocytes 
$(12,13)$, and their T cells more readily produce IL-2 and IL-22 upon re-stimulation $(14,15)$. Recent work has also shown that patients with PsA have higher levels of auto-antibodies directed against two previously identified putative auto-antigens of Pso, namely (carbamylated) LL37 and ADAMTSL5 $(16,17)$. So far, serum-based biomarker studies revealed elevated levels of high-sensitivity C-reactive protein, pro-inflammatory cytokines (e.g. IL-6, IL-33, TNF-a), adipokines and changes in markers of bone/cartilage damage in the Pso patients with PSA (18-27).

Overall, there is a scarcity of head-to-head serum biomarker comparisons in welldefined cohorts of Pso and PsA. The current study measured serum biomarkers in the early stage of PSA as compared to Pso matched for skin disease severity. We used a novel high-throughput proteomic platform capable of screening over 950 proteins in a small volume of serum. Previously, this technology proved valuable in providing new mechanistic insights into the pathogenesis of immune-mediated diseases of skin $(28,29)$, but results have not yet been reported in patients with rheumatic disease. The goal was to determine whether this biomarker platform could identify novel serum protein disturbances in PSA as compared to HC, Pso and AS (non-psoriatic reference group), and to specify which proteins best reflected major skin and joint manifestations.

\section{METHODS}

\section{Study design}

This study was performed at the University Medical Centre Utrecht (UMCU) and conducted in compliance with the Helsinki principles. Ethical approval was obtained from the institutional review board and all patients signed written informed consent before participation. Clinical parameters and serum samples were collected from a cohort of patients with Pso, PsA and AS as part of larger prospective observational study performed at the outpatient clinic of the Department of Rheumatology and Clinical Immunology.

For this study 79 patients were recruited. The Pso cohort (Pso, n=20) included patients with a dermatologist-confirmed diagnosis of Pso in whom concomitant PsA was clinically excluded by a rheumatologist (in training). Patients with psoriatic arthritis (PsA, $n=20$ ) fulfilled CIASsification of Psoriatic ARthritis (CASPAR) criteria (30). Patients with a clinical diagnosis of AS $(n=19)$, all without a history of Pso, were included as a non-psoriatic reference group. Serum samples were collected from healthy controls $(\mathrm{HC}, \mathrm{n}=20)$ from the UMCU. 


\section{Serum proteomic analysis}

Serum samples were collected, centrifuged at $1700 \mathrm{~g}$ for $10 \mathrm{~min}$ at $4^{\circ} \mathrm{C}$ and stored directly at $-80^{\circ} \mathrm{C}$. Frozen serum aliquots were shipped on dry ice to the Olink Facility (Uppsala, Sweden) without prior thawing and measured according to manufacturer's instructions as previously published (31). The Olink highthroughput proteomic platform employs a proximity extension-assay technology, in which oligonucleotide-labeled antibody pairs bind to a protein target. DNA reporter molecules bind to these antibodies, and are amplified to provide relative protein concentrations. One serum aliquot of $250 \mu \mathrm{l}$ was used to run 11 different Olink platform 'panels' encompassing 1012 proteins, some of which were run in more than one panel (panels: CARDIOMETABOLIC, CARDIOVASCULAR II, CARDIOVASCULAR III, CELL REGULATION, DEVELOPMENT, IMMUNE RESPONSE, INFLAMMATION, METABOLISM, NEUROLOGY, ONCOLOGY II, and ORGAN DAMAGE). Only data that passed OLINK internal quality control (QC) were used for analysis,. We removed samples entirely if they did not pass Olink internal quality control in $>80 \%$ of the data. We removed proteins entirely if they were below the limit of assay detection in $>40 \%$ of the samples. Some proteins were measured in multiple panels, in which case the protein data with the fewest missing values after quality control was used for analysis.

\section{Statistical approach}

For analysis of clinical characteristics, contingency analysis of two groups were performed using Chi-squared tests for categorical variables, and independent samples T-tests or Mann-Whitney $U$ tests for continuous variables. Contingency analysis of more than two groups were conducted with one-way independent ANOVA or Kruskal-Wallis for continuous variables, and with Chi square test for categorical variables. Spearman's rank correlation was used to correlate disease activity parameters to protein levels. Unless otherwise stated, a P-value of $<0.05$ was considered statistically significant.

The statistical analysis of proteomic data was performed on protein data received by OLINK without further normalization (quantile normalization did not impact the overall results, data not shown). OLINK protein data is expressed as an arbitrary unit (Normalized Protein expression, 'NPX') representing the relative protein concentration based on a log2 scale (i.e. absolute protein quantity cannot be compared across different proteins). Protein levels were compared between groups based on the likelihood ratio test and considered statistically significant at an FDR-corrected P-value of $<0.05$, referred to as differentially expressed proteins (DEPs). Analysis was performed to compare two groups (e.g. HC vs PsA) or to compare multiple groups (HC, Pso, PsA, AS), as specified in the text. Hierarchical 


\section{CHAPTER 7}

cluster analysis was based on Ward's method to create heatmaps (R pheatmap package, version 1.0.12). Classical multidimensional scaling (MDS) was performed with $\mathrm{R}$ built-in 'stats' package (cmdscale function), using the Euclidean distance matrix between samples based on protein data. The hierarchical cluster analysis and multidimensional scaling were performed using DEPs between groups based on a nominal P-value $<0.05$. The protein data shown in figures of hierarchical cluster analysis underwent Z-score normalization for the sake of visualization in heatmaps. Venn diagrams were modified from web-based BioVenn tool (32). Reactome pathway and Kyoto Encyclopedia of Genes and Genomes (KEGG) pathway enrichment analysis for DEPs was performed based on hypergeometric test using ReactomePA package (version 1.28.0) and clusterProfiler package (version 3.12.0), respectively. Statistical analysis was performed in R (version 3.6) and SPSS (version 25, SPSS Inc., Chicago IL, USA).

\section{RESULTS}

\section{Cohort description}

Clinical characteristics of the study participants are shown in Table 1. The Pso and PsA groups were matched for age, gender, and PASI score. The PsA cohort was recruited early after disease onset, typically with less than one year of disease duration. Except for two patients with PsA, none of the study participants were being treated with DMARDs. Following quality control (see methods), a total of 951 unique proteins and 77 samples (18 Pso, 20 PsA, 19 AS, 20 HC) were retained for further analysis.

Table 1. Baseline characteristics

\begin{tabular}{lcccc}
\hline & $\begin{array}{c}\mathbf{H C} \\
(\mathbf{N}=\mathbf{2 0})\end{array}$ & $\begin{array}{c}\text { Pso } \\
(\mathbf{N}=\mathbf{1 8})\end{array}$ & $\begin{array}{c}\text { PsA } \\
(\mathbf{N}=\mathbf{2 0})\end{array}$ & $\begin{array}{c}\text { AS } \\
(\mathbf{N}=\mathbf{1 9})\end{array}$ \\
\hline Age (years) & $43 \pm 13$ & $37 \pm 15$ & $41 \pm 9$ & $40 \pm 12$ \\
Female, n (\%) & $7(35)$ & $7(39)$ & $7(35)$ & $5(26)$ \\
BMI (kg/m2) & - & $29.9 \pm 7.9$ & $27.7 \pm 4.5$ & $24.2 \pm 3.4^{*}$ \\
Smoker, n (\%) & - & $6(40)$ & $7(35)$ & $2(11)$ \\
Disease duration (years) & - & $12.4(6.1-18.6)$ & $20.0(7.2-31.4)$ & - \\
- Psoriasis & - & - & $0.7(0.1-8.3)$ & - \\
- Psoriatic arthritis & - & - & - & $5.6(0.4-13.4)$ \\
- Ankylosing spondylitis & - & $1(6)^{*}$ & $11(55)$ & $11(58)$ \\
NSAID use, n (\%) & - & $0(0)$ & $2(10)$ & $0(0)$ \\
DMARD use, n (\%) & & & & \\
\hline
\end{tabular}




\begin{tabular}{|c|c|c|c|c|}
\hline & $\begin{array}{c}\mathrm{HC} \\
(\mathrm{N}=20)\end{array}$ & $\begin{array}{c}\text { Pso } \\
(\mathrm{N}=18)\end{array}$ & $\begin{array}{c}\mathrm{PsA} \\
(\mathrm{N}=20)\end{array}$ & $\begin{array}{c}\text { AS } \\
(N=19)\end{array}$ \\
\hline CRP (mg/L) & - & $2.8(1-6)$ & $2.8(2-4)$ & $3.2(1-7)$ \\
\hline ESR (mm/hour) & - & $5(2-8)$ & $5(2-13)$ & $5(3-14)$ \\
\hline \multicolumn{5}{|l|}{ Psoriasis indices } \\
\hline - PASI & - & $2.7(2-7)$ & $3.0(1-6)$ & - \\
\hline - Nail involvement, $\mathrm{n}(\%)$ & - & $9(56.3)$ & $13(72)$ & - \\
\hline - Vulgaris type only, n (\%) & - & $12(67)$ & $14(78)$ & - \\
\hline \multicolumn{5}{|l|}{ SpA manifestations } \\
\hline - Swollen joint count, of 76 & - & - & $3(1-9)^{*}$ & $0(0-0)$ \\
\hline - Tender joint count, of 78 & - & - & $3(1-10)^{*}$ & $0(0-0)$ \\
\hline - Dactylitis ever, n (\%) & - & - & $7(35)^{*}$ & $0(0)$ \\
\hline - Enthesitis, n (\%) & - & - & $9(47)^{\star}$ & $3(16)$ \\
\hline - Inflammatory back pain, n (\%) & - & - & $3(15)^{\star}$ & $19(100)$ \\
\hline - BASDAI & - & - & - & $4.2(2-5)$ \\
\hline
\end{tabular}

*Significant (P value $<0.05)$. Presented data are from time of baseline visit, unless otherwise indicated. Categorical data are presented with frequencies (\%) and continuous data are shown as mean \pm standard deviation (normally distributed variables) or median (interquartile range)(non-normally distributed variables). Abbreviations: AS: ankylosing spondylitis; BMI: body mass index; CRP: C-reactive protein; BASDAI: Bath ankylosing spondylitis disease activity index (range 0-10); DMARD: disease modifying antirheumatic drug use (past three months); ESR: erythrocyte sedimentation rate; HC: healthy control, NSAID use: non-steroidal anti-inflammatory drug use daily on stable dose; PASI: psoriasis area and severity index (range 0-72); PsA: psoriatic arthritis, Pso: psoriasis; SpA: spondyloarthritis.

\section{Major proteins changes in PsA serum compared to HC serum}

We first set out to specifically compare the serum of PsA to HC and found 68 differentially expressed proteins (DEPS)(FDR-corrected $\mathrm{P}<0.05)$ (supplementary Table S1). Most of the top DEPs between PsA and HC have not previously been implicated in the pathogenesis of PsA, which included proteins such as ANXA1, ADAM 23, and VIM (supplementary Fig. S1A). Hierarchical cluster analysis revealed that the serum proteomic profile of PsA patients could be clearly distinguished from the serum proteomic profile of HC (supplementary Fig. S1B). 


\section{Common and unique protein disturbances in serum of PsA}

We first examined whether those serum proteins changes were unique to PsA, or if they were also dysregulated in Pso and/or AS. Of the 68 DEPs between PsA and HC, 48 proteins (71\%) were also dysregulated in Pso and/or AS (Fig. 1A). The most significant DEPs between the groups were proteins that all had higher serum levels in patient groups as compared to HC (Fig. 1B). This list again included the proteins ANXA1, VIM and TOP2B. In total 20 proteins (29\%) were dysregulated in PsA as compared to $\mathrm{HC}$, which were not dysregulated in AS or Pso as compared to $\mathrm{HC}$ (Fig. 1C). This list included proteins ADAM 23, Neurogenic locus notch homologue protein 3 (Notch 3), and SLITRK6. Interestingly, many of the proteins in this list were lower in serum of PsA as compared to HC.

We next compared patient groups directly. Importantly, there were no DEPs when directly comparing PsA to Pso based on FDR-corrected $p<0.05$. An exploratory analysis (based on nominal P-value) comparing PsA to Pso can be found in supplementary Fig. S2A and B. We found that CLEC4A and SOD1 were the only proteins significantly different between patient groups, being elevated in AS (supplementary Fig. S3). Some specific proteins that have previously been implicated in the pathogenesis of these disease are displayed in supplementary Fig. S4. The list of DEPs can be found in Sup Tables 1-4. Taken together, we identified 20 proteins uniquely dysregulated in PsA, while the majority of proteins disturbances were also dysregulated in Pso and/or AS. 


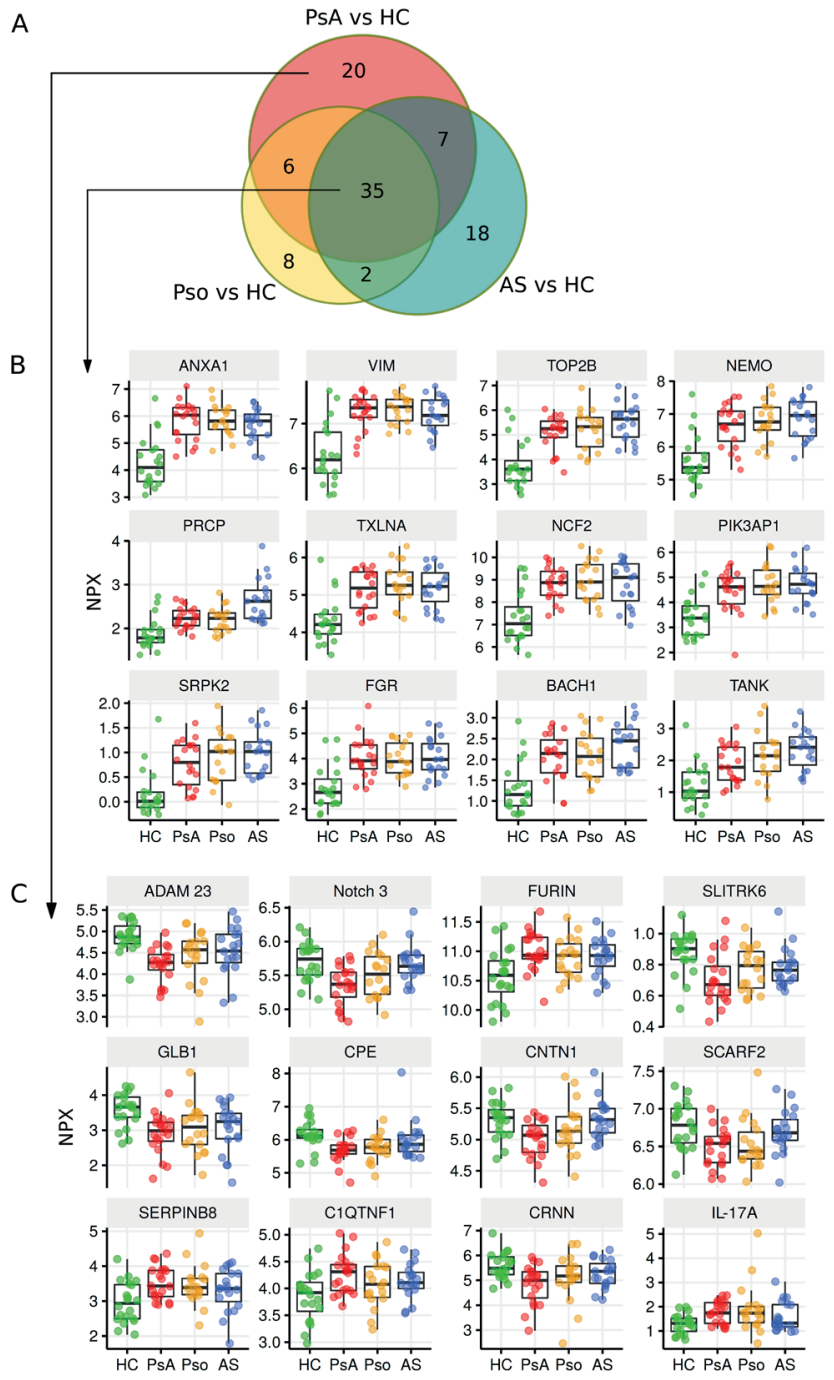

Fig. 1. Common and unique protein disturbances in serum of PsA (A) Overlap in DEPs between patient groups vs HC. This Venn diagram shows the number of DEPs between each patient group as compared with HC. For example, there were 68 DEPs when comparing PsA with HC, of which 35 proteins were also differentially expressed when comparing Pso with $\mathrm{HC}$ or comparing AS with HC. Results are based on FDRcorrected $P$-value $<0.05$. (B) Common DEPs in all patient groups. Thirty-five proteins were differentially expressed in all patient groups as compared with HC. The 12 most significant proteins are displayed as boxplots (DEPs with the lowest FDR-corrected $P$-value). (C) DEPs only found in PsA vs HC. Twenty proteins were differentially expressed in PSA compared with $\mathrm{HC}$, but not dysregulated in other patient groups compared with HC. The 12 most significant proteins are displayed in boxplots (DEPs with the lowest FDR-corrected P-value). HC: healthy control; Pso: psoriasis; NPX: Normalized Protein eXpression; DEPs: differentially expressed proteins; FDR: false discovery rate. 


\section{Overall serum proteomic signature is similar in PsA and Pso}

Hierarchical cluster analysis showed that most patients, regardless of diagnosis, clustered separately from HC. The serum proteomic profile of PsA patients grouped closer to the Pso patients than to the AS patients (Fig. 2A). Using an alternative method of analyzing the data, namely multidimensional scaling (MDS) analysis, we also found that $\mathrm{HC}$ grouped separately from patients, and that PSA and Pso grouped close together (Fig. 2B). Finally, pathway enrichment analysis on the sets of DEPs between patient groups versus HC similarly revealed that very similar pathways were enriched in PsA and Pso (supplementary Fig. S5).

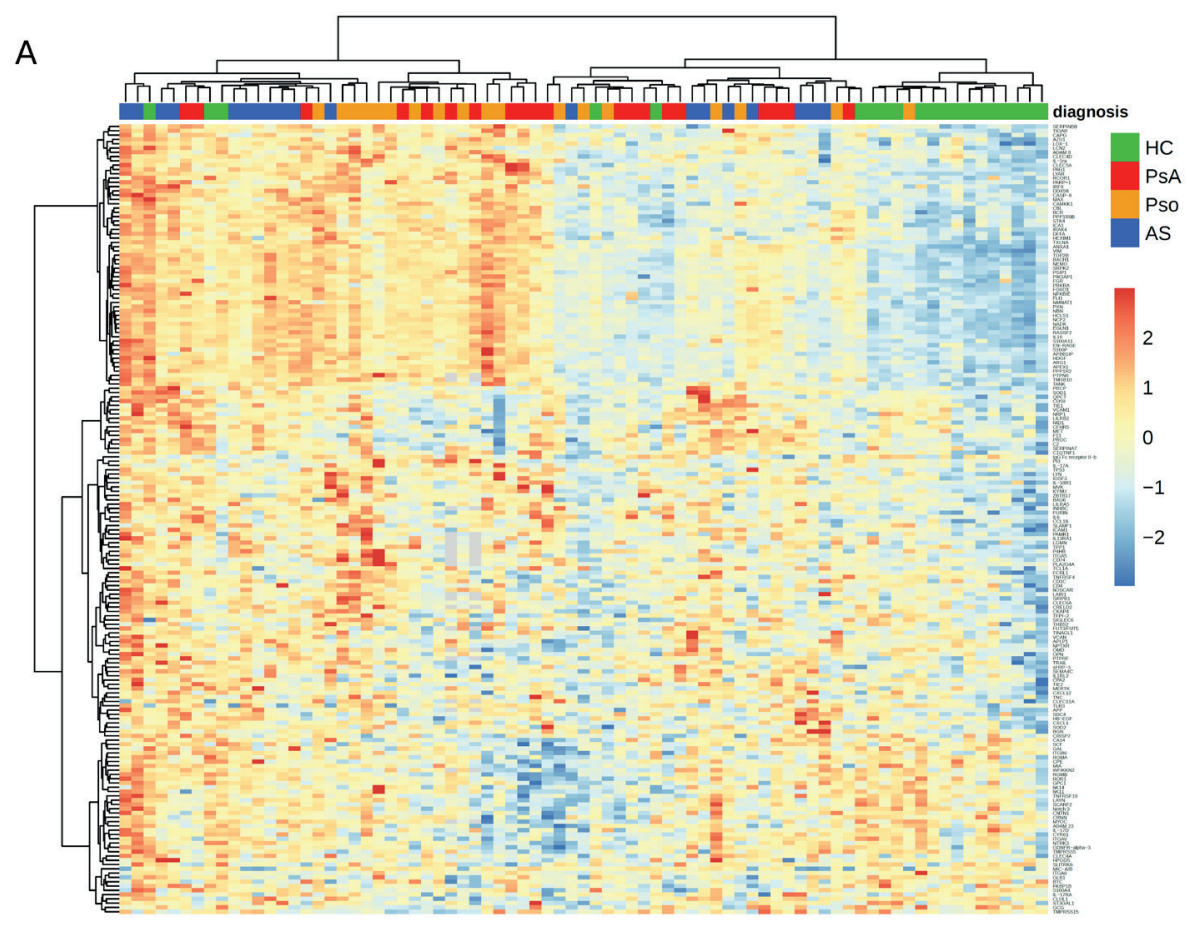


B

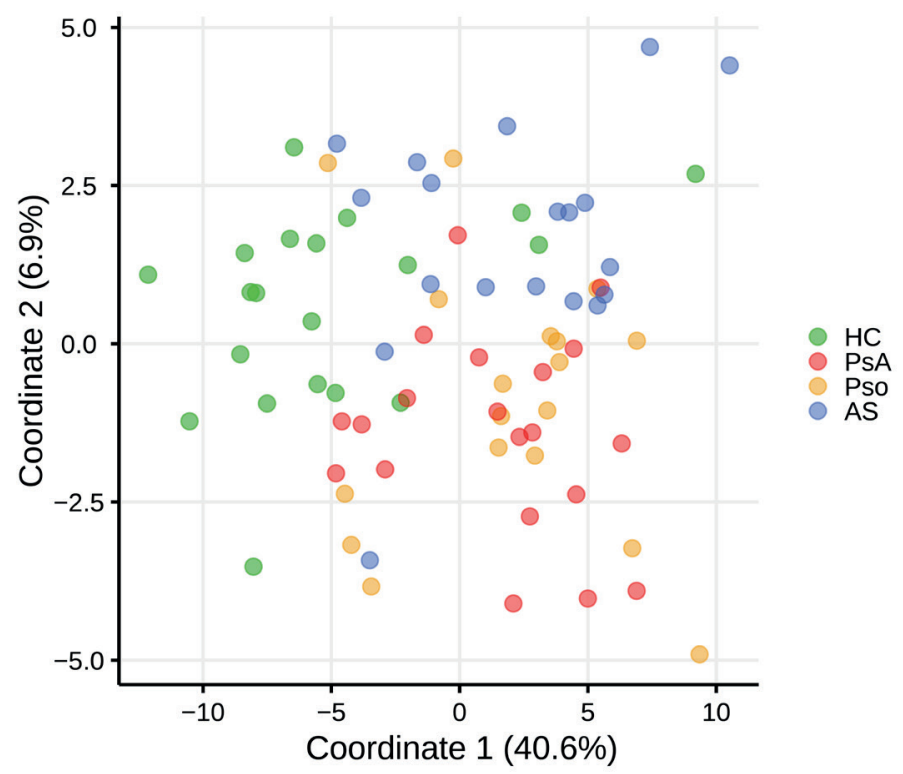

Fig. 2 Overall serum proteomic signature is similar in PsA and Pso (A) Serum in Pso and PsA overlap based on hierarchical clustering analysis. Hierarchical clustering shown in the heatmap reveals HC cluster separately from the different patient groups. The clustering also reveals that most Pso and PsA patients cluster separately from AS patients. This analysis is based on DEPs with nominal $P$-value $<0.05$ when comparing all groups. (B) Serum in Pso and PSA overlap based on MDS. The MDS plot reveals that HC cluster separately from the different patient groups. Similar to the hierarchical clustering, Pso and PSA tend to cluster separately from AS. This analysis is based on DEPs with nominal $P$-value $<0.05$ when comparing all groups. HC: healthy control; Pso: psoriasis; DEPs: differentially expressed proteins; MDS: multidimensional scaling.

\section{Proteins reflecting joint and skin disease activity}

We next examined which serum proteomic changes best reflected the major disease manifestations with respect to joint and skin disease activity in patients with PsA and Pso. The number of swollen joints (SJC) had the strongest positive correlation to Intracellular adhesion molecule 1 (ICAM-1; $r=0.81, p<0.001$ ), C-C motif chemokine 18 (CCL18; $r=0.76, \mathrm{P}<0.001$ ) and dipeptidyl peptidase 4 (DPP4; $r=$ $0.75, \mathrm{P}<0.001)$, whereas swollen joint count had the strongest negative correlation to VEGFD ( $r=-0.73, P<0.001)$ (Fig. 3). 


\section{CHAPTER 7}

When PsA and Pso patients were considered as one group (data pooled together), PASI scores had the strongest correlation to the proteins PI3 $(r=0.54, P<0.001)$, IL17 receptor $A(r=-0.51, P<0.01)$, MMP-1 $(r=0.47, P=0.01)$ and SERPINB8 $(r=0.46$, $P<0.01)$. Surprisingly, there were more proteins that correlated to PASI score when analyzing the Pso and PsA cohorts separately as compared to analyzing the Pso and PsA patients pooled together (Fig. 4). PASI score was correlated to Gal-4 ( $r=$ $-0.72, P<0.001)$ and IGFBPL1 $(r=-0.65, P<0.01)$ but only in patients with PSA. PASI score was correlated to PD-L2 $(r=0.68, P<0.01)$ and MSR1 $(r=0.67, P<0.01)$ but only in patients with Pso (Fig. 4).
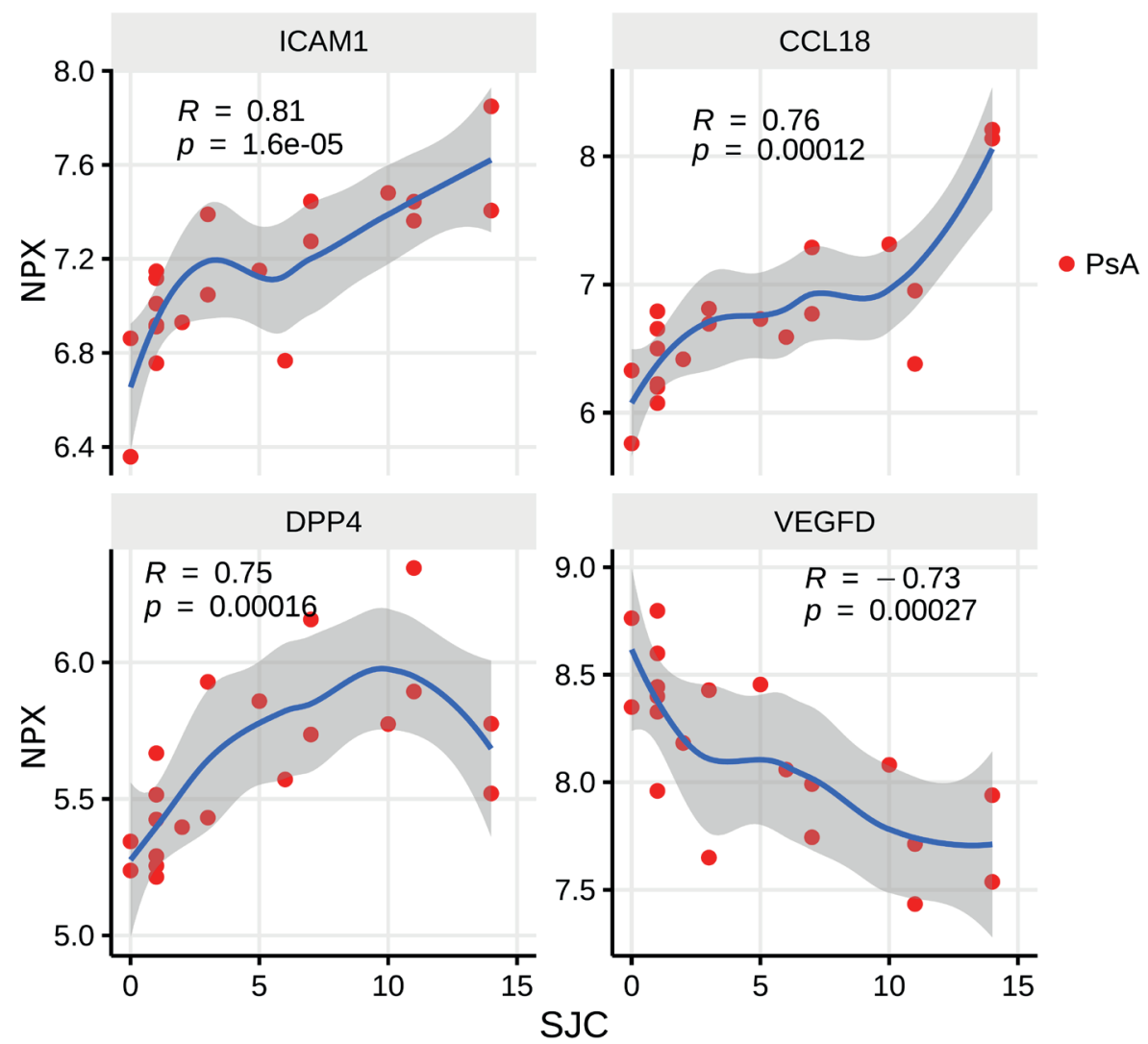

Fig. 3 Top proteins related to arthritis activity. The swollen joint count (SJC) vs relative protein levels of ICAM-1, CCL18, DPP4 and VEGFD. Spearman's rank correlation (R) and P-value in PSA are displayed in the figure. Locally estimated scatterplot smoothing (loess) curve is shown. NPX: Normalized Protein eXpression; ICAM-1: Intracellular adhesion molecule 1; CCL18: CC motif chemokine 18; DPP4: Dipeptidyl peptidase 4 . 

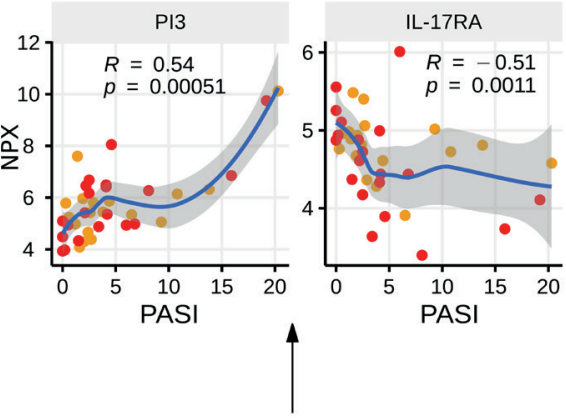

PAS
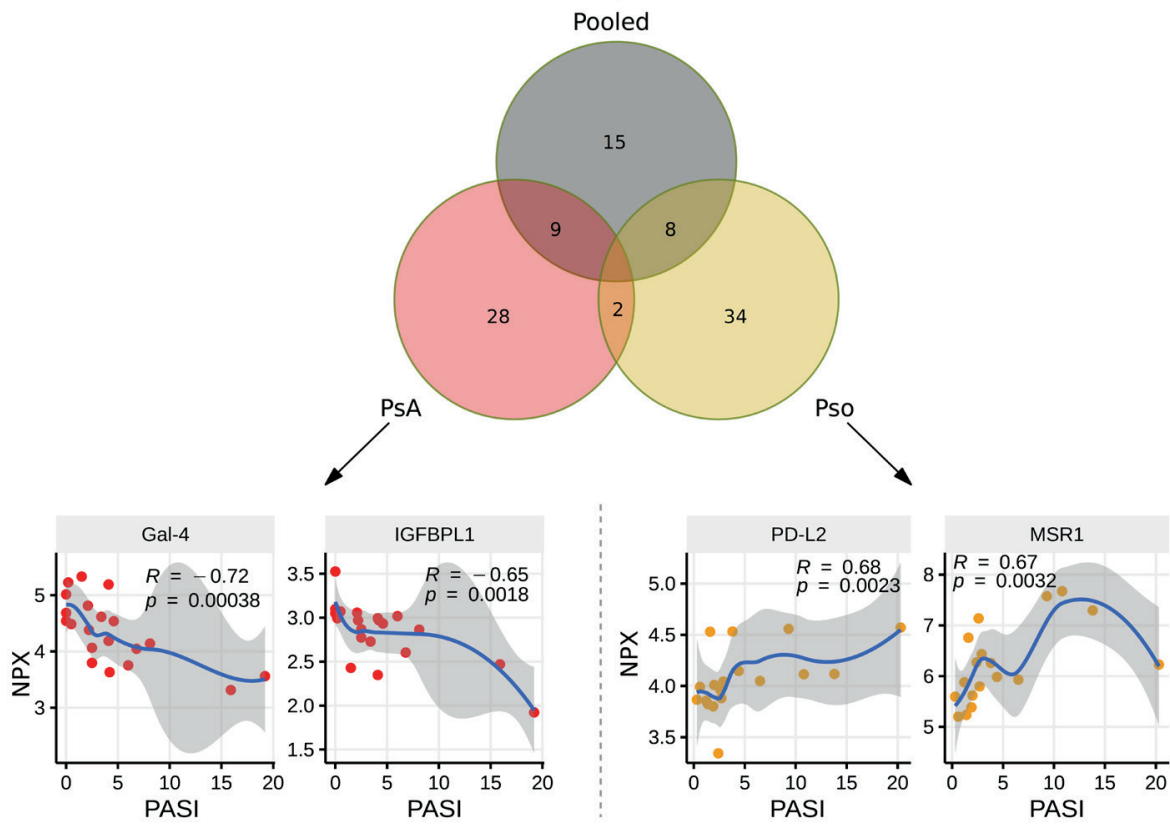

Fig. 4 Top proteins related to Pso activity. The Venn diagram shows the total number of proteins that significantly correlate to PASI score (nominal $P$-value $<0.05$ ). The analysis was performed when pooling PsA and Pso patients (grey circle), taking PsA patients only (red circle) or taking Pso patients only (orange circle). The PASI was correlated to relative protein levels. Spearman's rank correlation (R) and $P$-value are shown in the figure. Locally estimated scatterplot smoothing (loess) curve is shown. Pso: psoriasis; PASI: Psoriasis Area and Severity Index; NPX: Normalized Protein eXpression; PI3: Elafin; IL-17RA: IL-17 receptor A; Gal-4: Galectin-4; IGFBPL1: Insulin-like growth factor-binding protein-like 1; PD-L2: Programmed cell death 1 ligand 2; MSR1: Macrophage scavenger receptor types I and II. 


\section{DISCUSSION}

This study found large proteomic disturbances in the serum of patients with PsA and revealed that the strongest proteomic changes occurred in novel proteins not yet linked to the pathogenesis of PsA. Importantly, the majority of protein changes in serum of patients with PsA were similarly disturbed in patients with Pso in whom PsA was excluded. From over 950 proteins screened, we were able to narrow down specific proteins of interest correlating to the major clinical manifestations of these diseases.

This is one of few head-to-head serum proteomic comparisons in a wellcharacterized cohort of patients with PsA and Pso. Our PsA cohort consisted of patients with early disease onset and was carefully matched to have similar clinical characteristics (including PASI score) to the Pso patients. From a clinical perspective, our results indicate that none of the evaluated serum proteins (singularly) is a likely candidate for a simple diagnostic biomarker capable of discriminating early PsA from Pso.In other words, a simple blood test to differentiate PsA from Pso may not be a feasible goal for daily clinical practice, at least not based on the proteins we evaluated. Instead, our results primarily contribute to the understanding of the pathogenesis of PSA-which includes specifying potential drug targets. From a pathophysiological perspective, our data support the ' 2 phenotypes of 1 disease' hypothesis $(8,9)$.

Our study adds important insight into the question as to which type of tissue samples is best suited to unravel the pathogenesis of PsA. PsA and Pso fall within a spectrum of diseases with shared genetic background and presumably shared immunologic drivers. From a clinician point of view, however, they are distinct: some patients develop (poly)arthritis which requires specific clinical intervention. Therefore, there must be specific drivers (local and/or systemic) within this overlapping psoriatic spectrum that enable the development of overt arthritis manifestations. Our broad analysis reveals that PsA and Pso are extremely difficult to discriminate based on serum proteomic changes, underscoring that other sites of the body, such as synovial tissue, should be an important target of future research. It will still be important to find methods of incorporating appropriate control groups, ideally Pso patients in whom PsA is excluded by a rheumatologist, even when studying tissue sites such as synovial tissue. Surprisingly, we found that many serum proteins were related to PASI score when dichotomizing the analysis for Pso only and PsA only. This may indicate there are different primary drivers of cutaneous inflammation and/or secondary systemic responses upon inflammation occurring in PSA compared to Pso. A comparison of the skin in PSA compared to Pso as tissue site has only been addressed in a small number studies and therefore warrants specific tissue comparisons $(33,34)$. 
We here identified specific proteins strongly associated with joint disease activity. ICAM-1 is a molecule important for trans-endothelial migration of leucocytes via interaction with LFA-1. ICAM-1 has previously been identified in the pathogenesis of Pso and PsA $(35,36)$. In rheumatoid arthritis (RA) synovial tissue it was shown that ICAM-1 expression marked a specific myeloid synovial tissue phenotype (37). Interestingly, previous attempts to target LFA-1 with monoclonal antibodies for the treatment of Pso lead to the new onset arthritis in many patients enrolling in the trials (38), supporting the notion that the balance of leucocyte extravasation mediated by ICAM-1 could be important in arthritis development. VEGFD is one of the members of the endothelial growth factors involved in angiogenesis and lymphangiogenesis in cancer, and while this specific family member has not been described in rheumatic disease (39), VEGF has been implicated in the pathogenesis of arthritis (40). Considering that we performed a broad, unbiased serologic screening our data again highlight the importance of angiogenesis in PsA, which is in agreement with existing histologic data in PsA showing increased angiogenesis to be an important feature of PsA synovial tissue $(7,35,41)$. Two additional proteins were strongly correlated to arthritis activity: CCL18 and DPP4. DPP4 (dipeptidyl peptidase 4) is currently a target for type 2 diabetes mellitus with still unclear role of DPP4 in development of arthritis (42). CCL18 is expressed by endothelial cells in the synovial tissue of RA and has been identified as disease activity marker in RA and other diseases (43).

A strength of our study is the broad set of protein panels we have measured. We hence observed that the strongest protein disturbances were not well-known cytokines and chemokines, but rather proteins not previously implicated in the pathogenesis of rheumatic disease, including ADAM 23 and Notch 3 . ADAM23 is a non-proteolytic member of the "A disintegrin and metalloproteases" (ADAM) family known for high expression in brain and role in neuronal differentiation, but also shown to inhibit cell adhesion and cell migration in cancer cells, possible via interaction with integrin av $\beta 3(44,45)$. Neurogenic locus notch homolog protein 3 (Notch 3 ) has very broad functions, and is aberrantly expression in psoriatic skin and was shown to modulate T-helper cell phenotypes function $(46,47)$. Our patient cohorts have an expected overlapping pathogenic spectrum (Pso, PsA, AS). Future studies should consider including other rheumatic diseases with more distinct clinical features and pathogenesis (e.g. gout and osteoarthritis) in order to further address the specificity of the protein changes. While the protein disturbances were not specific to PsA, this per se does not preclude their importance in pathogenesis or their role as potential therapeutic target: many of the current therapeutics (e.g. TNF-alpha-inhibitors) are effective across a range of distinct clinical entities considered to be driven by different pathways. 
Some of the more familiar proteins changes included IL- 6 and IL-17A, which are known drug targets for rheumatologic diseases. Studies in RA highlight that serum levels of cytokines are unlikely to predict clinical response to monoclonal antibodies targeting that respective cytokine $(48,49)$. Nevertheless, we detected elevated levels of IL- 6 in PSA and also found a positive correlation between IL- 6 levels and joint disease activity measures, which supports current efforts examining IL-6 as a potential therapeutic target for patients with PsA.

Our study was designed to recruit PsA patients without DMARDs and early after disease onset resulting in PSA patients with mostly oligoarthritis. The serum proteomic results best represent the oligoarthritis pattern in PsA, but our cohort does not represent the entire spectrum of PsA patients, i.e. those with very severe polyarticular disease. Our choice to avoid patients with DMARDs is underscored by recent data using the same proteomic platform in Pso patients confirming that most proteins undergo vast changes upon initiation of immunomodulatory drugs (29).

A limitation of the current study is the relatively small cohort size, which means that we may have underestimated the number of proteins that are different between Pso and PsA groups due to stringent FDR-correction. Realistically, it is challenging to include large numbers of patients in basic science studies with very severe disease that are not (yet) treated with immunomodulatory drugs. Clearly, it will be necessary to (i) replicate the major protein disturbances identified by our screening and (ii) determine if the proteins are downstream biomarkers of the disease or directly involved in the pathogenesis. Functional validation will be necessary to determine which of these specific factors or combination of factors contributes to the pathogenesis of PSA.

To overcome some of the aforementioned challenges we recommend that, similar to sharing gene expression data, these proteomic datasets can be publicly shared (e.g. repositories). Firstly, this provides additional scientific transparency of the results. Secondly, by sharing datasets the proteins can be compared across diseases (determine specificity) and allow for rapid validation and identification of those proteins worth pursuing for in-vitro experiments. These collaborative efforts should maximize the yield of costly scientific endeavors, whilst ensuring acknowledgement of data in a competitive scientific landscape.

In summary, we have identified novel serum protein disturbances in PSA and furthermore establish that both Pso patients and PsA patients with oligoarthritis have an overall shared serum proteomic signature. 


\section{Acknowledgements}

We would like to thank the patients for participating in the study. We would like to thank the clinical study team (Nienke Kleinrensink, Nanette Vincken, Anne Karien Marijnissen, Anneloes van Loo, Karin Schrijvers and Joke Nijdeken). W.T. was supported by the China Scholarship Council (CSC) No. 201606300050.

Funding: This study was in part funded by Janssen. This study was in part funded by Health Holland, Top Sector Life Sciences \& Health.

Disclosure statement: T.R. received consultancy fees from Jansen in 2016 and 2017 on topics that were unrelated to the content of this manuscript. T.R. is currently an employee of AbbVie, with no conflicts of interest regarding the work of this manuscript. D.B. received consultancy fees from Janssen in 2018 and 2019 on topics that were unrelated to the content of this manuscript. Ernesto Munoz-Elias and Samuel DePrimo are Janssen R\&D LLC employees. The other authors have declared no conflicts of interest. 


\section{References}

1. Ritchlin CT, Colbert RA, Gladman DD. Psoriatic Arthritis. N Eng/J Med 2017;376:957-970.

2. Kavanaugh A, Gottlieb A, Morita A, Merola JF, Lin C-Y, Birt J, et al. The contribution of joint and skin improvements to the health-related quality of life of patients with psoriatic arthritis: a post hoc analysis of two randomised controlled studies. Ann Rheum Dis 2019.

3. Furue $\mathrm{K}$, Ito T, Furue M. Differential efficacy of biologic treatments targeting the TNF-a/IL-23/ IL-17 axis in psoriasis and psoriatic arthritis. Cytokine 2018;111:182-188.

4. Belasco J, Louie JS, Gulati N, Wei N, Nograles K, Fuentes-Duculan J, et al. Comparative genomic profiling of synovium versus skin lesions in psoriatic arthritis. Arthritis Rheumatol 2015;67:934944.

5. Krueger JG, Kirkham B, Ritchlin CT. Basic and Translational Science: A Report from the GRAPPA 2016 Annual Meeting.J Rheumatol 2017;44:679-683.

6. Ospelt C, Frank-Bertoncelj M. Why location matters - site-specific factors in rheumatic diseases. Nat Rev Rheumatol 2017;13:433-442.

7. Veale DJ, Fearon U. The pathogenesis of psoriatic arthritis. Lancet (London, England) 2018;391:2273-2284.

8. Boehncke W-H. Psoriasis and Psoriatic Arthritis: Flip Sides of the Coin? Acta Derm Venereol 2016;96:436-41.

9. Sakkas LI, Bogdanos DP. Are psoriasis and psoriatic arthritis the same disease? The IL-23/IL17 axis data. Autoimmun Rev 2017;16:10-15.

10. Bowes J, Budu-Aggrey A, Huffmeier U, Uebe S, Steel K, Hebert HL, et al. Dense genotyping of immune-related susceptibility loci reveals new insights into the genetics of psoriatic arthritis. Nat Commun 2015;6:6046.

11. Stuart PE, Nair RP, Tsoi LC, Tejasvi T, Das S, Kang HM, et al. Genome-wide Association Analysis of Psoriatic Arthritis and Cutaneous Psoriasis Reveals Differences in Their Genetic Architecture. Am J Hum Genet 2015;97:816-836.

12. Pollock RA, Abji F, Liang K, Chandran V, Pellett FJ, Virtanen C, et al. Gene expression differences between psoriasis patients with and without inflammatory arthritis. I Invest Dermatol 2015;135:620-623.

13. Abji F, Pollock RA, Liang K, Chandran V, Gladman DD. Brief Report: CXCL10 Is a Possible Biomarker for the Development of Psoriatic Arthritis Among Patients With Psoriasis. Arthritis Rheumatol (Hoboken, NJ) 2016;68:2911-2916.

14. Capsoni F, Molteni S, Raeli L, Diani M, Altomare a., Garavaglia M, et al. Differential expression of interleukin-2 by anti-CD3-stimulated peripheral blood mononuclear cells in patients with psoriatic arthritis and patients with cutaneous psoriasis. Clin Exp Dermatol 2014;39:385-390.

15. Benham H, Norris P, Goodall J, Wechalekar MD, Fitzgerald O, Szentpetery A, et al. Th17 and Th22 cells in psoriatic arthritis and psoriasis. Arthritis Res Ther 2013;15:R136.

16. Yuan Y, Qiu J, Lin Z-T, Li W, Haley C, Mui UN, et al. Identification of Novel Autoantibodies Associated With Psoriatic Arthritis. Arthritis Rheumatol (Hoboken, NJ) 2019;71:941-951. 
17. Frasca L, Palazzo R, Chimenti MS, Alivernini S, Tolusso B, Bui L, et al. Anti-LL37 Antibodies Are Present in Psoriatic Arthritis (PsA) Patients: New Biomarkers in PsA. Front Immunol 2018;9:1936.

18. Chandran V, Cook RJ, Edwin J, Shen H, Pellett FJ, Shanmugarajah S, et al. Soluble biomarkers differentiate patients with psoriatic arthritis from those with psoriasis without arthritis. Rheumatology 2010;49:1399-1405.

19. Alenius G-M, Eriksson C, Rantapää Dahlqvist S. Interleukin-6 and soluble interleukin-2 receptor alpha-markers of inflammation in patients with psoriatic arthritis? Clin Exp Rheumatol 27:120-3.

20. Xue Y, Jiang L, Cheng Q, Chen H, Yu Y, Lin Y, et al. Adipokines in Psoriatic Arthritis Patients: The Correlations with Osteoclast Precursors and Bone Erosions. PLoS One 2012;7:1-11.

21. Li J, Liu L, Rui W, Li X, Xuan D, Zheng S, et al. New Interleukins in Psoriasis and Psoriatic Arthritis Patients: The Possible Roles of Interleukin-33 to Interleukin-38 in Disease Activities and Bone Erosions. Dermatology 2017.

22. Dalbeth N, Pool B, Smith T, Callon KE, Lobo M, Taylor WJ, et al. Circulating mediators of bone remodeling in psoriatic arthritis: implications for disordered osteoclastogenesis and bone erosion. Arthritis Res Ther 2010;12:R164.

23. Bartosińska J, Michalak-Stoma A, Juszkiewicz-Borowiec M, Kowal M, Chodorowska G. The Assessment of Selected Bone and Cartilage Biomarkers in Psoriatic Patients from Poland. Mediators Inflamm 2015;2015:194535.

24. Cubillos S, Krieg N, Norgauer J. Effect of Vitamin D on Peripheral Blood Mononuclear Cells from Patients with Psoriasis Vulgaris and Psoriatic Arthritis. Heymann D, ed. PLoS One 2016;11:e0153094.

25. Amin TE, ElFar NN, Ghaly NR, Hekal MM, Hassan AM, Elsaadany HM. Serum level of receptor activator of nuclear factor kappa-B ligand in patients with psoriasis. Int J Dermatol 2016;55:e227-33.

26. Kim DS, Shin D, Lee MS, Kim HJ, Kim DY, Kim SM, et al. Assessments of neutrophil to lymphocyte ratio and platelet to lymphocyte ratio in Korean patients with psoriasis vulgaris and psoriatic arthritis. J Dermatol 2016;43:305-10.

27. Mitsui A, Tada Y, Takahashi T, Shibata S, Kamata M, Miyagaki T, et al. Serum IL-33 levels are increased in patients with psoriasis. Clin Exp Dermatol 2016;41:183-9.

28. Brunner PM, Suárez-Fariñas M, He H, Malik K, Wen H-C, Gonzalez J, et al. The atopic dermatitis blood signature is characterized by increases in inflammatory and cardiovascular risk proteins. Sci Rep 2017;7:8707.

29. Kim J, Tomalin L, Lee J, Fitz LJ, Berstein G, Correa-da Rosa J, et al. Reduction of Inflammatory and Cardiovascular Proteins in the Blood of Patients with Psoriasis: Differential Responses between Tofacitinib and Etanercept after 4 Weeks of Treatment. J Invest Dermatol 2018;138:273-281.

30. Taylor W, Gladman D, Helliwell P, Marchesoni A, Mease P, Mielants H. Classification criteria for psoriatic arthritis: development of new criteria from a large international study. Arthritis Rheum 2006;54:2665-73. 


\section{CHAPTER 7}

31. Assarsson E, Lundberg M, Holmquist G, Björkesten J, Thorsen SB, Ekman D, et al. Homogenous 96-plex PEA immunoassay exhibiting high sensitivity, specificity, and excellent scalability. PLoS One 2014;9:e95192.

32. Hulsen T, Vlieg J de, Alkema W. BioVenn - a web application for the comparison and visualization of biological lists using area-proportional Venn diagrams. BMC Genomics 2008;9:488.

33. Veale DJ, Barnes L, Rogers S, FitzGerald O. Immunohistochemical markers for arthritis in psoriasis. Ann Rheum Dis 1994;53:450-4.

34. Šahmatova $L$, Sügis $E$, Šunina $M$, Hermann $H$, Prans $E$, Pihlap $M$, et al. Signs of innate immune activation and premature immunosenescence in psoriasis patients. Sci Rep 2017;7:7553.

35. Veale D, Yanni G, Rogers S, Barnes L, Bresnihan B, Fitzgerald O. Reduced synovial membrane macrophage numbers, ELAM-1 expression, and lining layer hyperplasia in psoriatic arthritis as compared with rheumatoid arthritis. Arthritis Rheum 1993;36:893-900.

36. Veale $\mathrm{D}$, Rogers $\mathrm{S}$, Fitzgerald $\mathrm{O}$. Immunolocalization of adhesion molecules in psoriatic arthritis, psoriatic and normal skin. BrJ Dermatol 1995;132:32-8.

37. Dennis G, Holweg CTJ, Kummerfeld SK, Choy DF, Setiadi AF, Hackney JA, et al. Synovial phenotypes in rheumatoid arthritis correlate with response to biologic therapeutics. Arthritis Res Ther 2014;16:R90.

38. Viguier M, Richette P, Aubin F, Beylot-Barry M, Lahfa M, Bedane C, et al. Onset of psoriatic arthritis in patients treated with efalizumab for moderate to severe psoriasis. Arthritis Rheum 2008;58:1796-802.

39. Stacker SA, Achen MG. Emerging Roles for VEGF-D in Human Disease. Biomolecules 2018;8.

40. Balogh E, Biniecka M, Fearon U, Veale DJ, Szekanecz Z. Angiogenesis in Inflammatory Arthritis. Isr Med Assoc J 2019;5:345-352.

41. Kruithof E, Baeten D, Rycke L De, Vandooren B, Foell D, Roth J, et al. Synovial histopathology of psoriatic arthritis, both oligo- and polyarticular, resembles spondyloarthropathy more than it does rheumatoid arthritis. Arthritis Res Ther 2005;7:R569-80.

42. Kim SC, Schneeweiss S, Glynn RJ, Doherty M, Goldfine AB, Solomon DH. Dipeptidyl peptidase-4 inhibitors in type 2 diabetes may reduce the risk of autoimmune diseases: a population-based cohort study. Ann Rheum Dis 2015;74:1968-75.

43. Lieshout AWT van, Fransen J, Flendrie M, Eijsbouts AMM, Hoogen FHJ van den, Riel PLCM van, et al. Circulating levels of the chemokine CCL18 but not CXCL16 are elevated and correlate with disease activity in rheumatoid arthritis. Ann Rheum Dis 2007;66:1334-8.

44. Hsia H-E, Tüshaus J, Brummer T, Zheng Y, Scilabra SD, Lichtenthaler SF. Functions of "A disintegrin and metalloproteases (ADAMs)" in the mammalian nervous system. Cell Mol Life Sci 2019;76:3055-3081.

45. Ota M, Mochizuki S, Shimoda M, Abe H, Miyamae $\mathrm{Y}$, Ishii K, et al. ADAM23 is downregulated in side population and suppresses lung metastasis of lung carcinoma cells. Cancer Sci 2016;107:433-43.

46. Ota T, Takekoshi S, Takagi T, Kitatani $\mathrm{K}$, Toriumi $\mathrm{K}$, Kojima $\mathrm{T}$, et al. Notch signaling may be involved in the abnormal differentiation of epidermal keratinocytes in psoriasis. Acta Histochem Cytochem 2014;47:175-83. 
47. Jiao Z, Wang W, Xu H, Wang S, Guo M, Chen Y, et al. Engagement of activated Notch signalling in collagen II-specific T helper type 1 (Th1)- and Th17-type expansion involving Notch3 and Delta-like1. Clin Exp Immunol 2011;164:66-71.

48. Cuppen BVJ, Welsing PMJ, Sprengers JJ, Bijlsma JWJ, Marijnissen ACA, Laar JM van, et al. Personalized biological treatment for rheumatoid arthritis: a systematic review with a focus on clinical applicability. Rheumatology (Oxford) 2016;55:826-39.

49. Romão VC, Fonseca JE. Major Challenges in Rheumatology: Will We Ever Treat Smarter, Instead of Just Harder? Front Med 2019;6:144. 


\section{Supplementary figures}

A
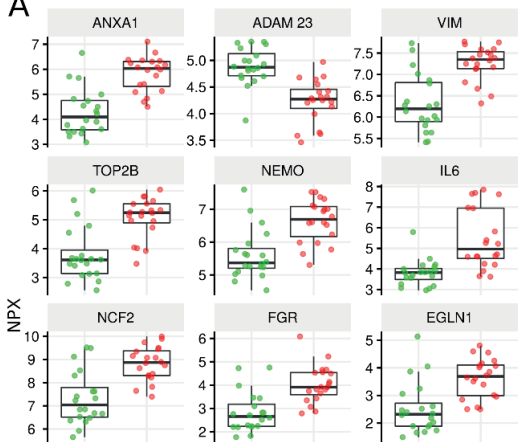

(6.)

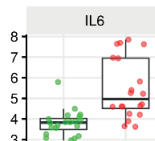

EGLN1
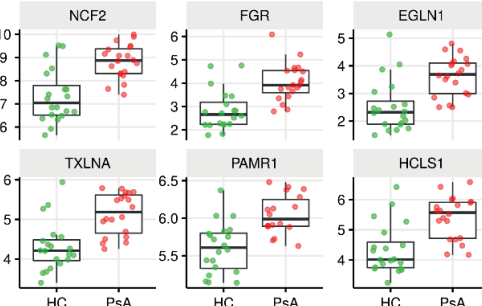

PAMR1

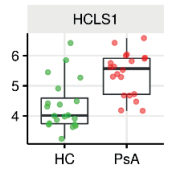

B

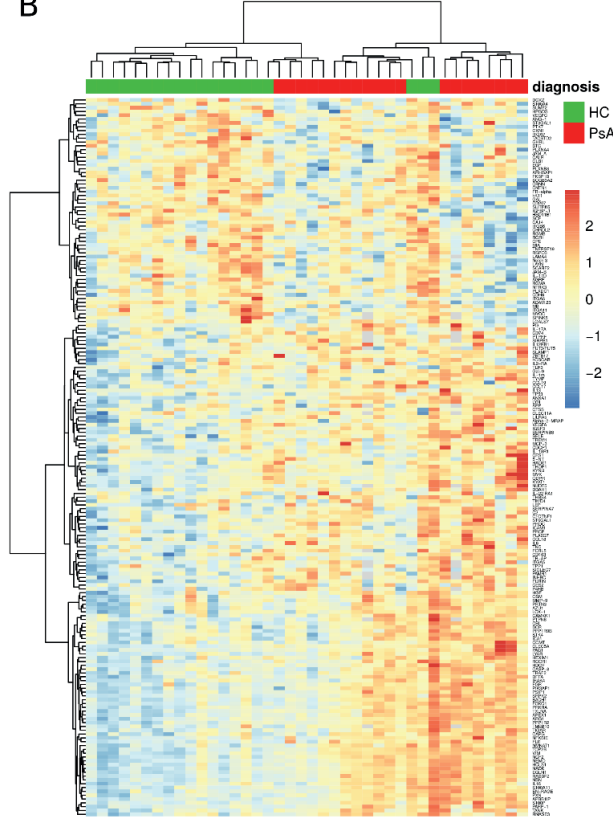

\section{Supplementary Figure S1: Top proteins differentiating PsA from HC}

1A. Top proteins differentiating PSA from HC. Boxplots show the twelve most significant proteins differentiating PsA from HC (DEPs with the lowest FDR-corrected P value).

1B. Hierarchical clustering differentiates HC from PsA. Hierarchical clustering is shown based on all of the DEPs when comparing PSA to HC (nominal P value $<0.05$ ).

Abbreviations: healthy control (HC); psoriatic arthritis (PsA); Normalized Protein expression (NPX); differentially expressed proteins (DEPs); Annexin A1 (ANXA1); Disintegrin and metalloproteinase domaincontaining protein 23 (ADAM 23); Vimentin (VIM); Neutrophil cytosol factor 2 (NCF2); DNA topoisomerase 2-beta (TOP2B); NF-kappa-B essential modulator (NEMO); Interleukin-6 (IL6); Tyrosine-protein kinase Fgr (FGR); Alpha-taxilin (TXLNA); Inactive serine protease PAMR1 (PAMR1); Hematopoietic lineage cell-specific protein (HCLS1); EgI nine homolog 1 (EGLN1) 
A

CPA2

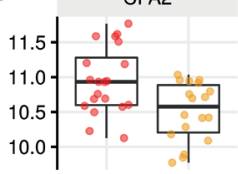

$\mathrm{CD} 48$
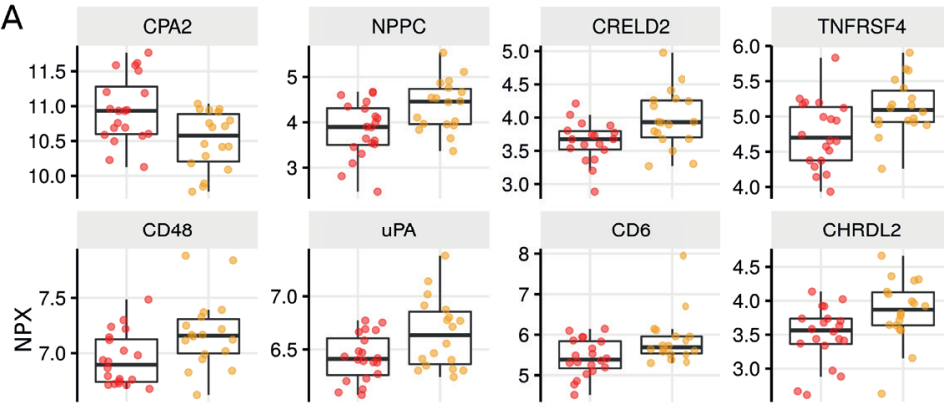

UPA

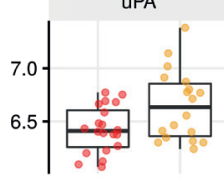

CD6

CHRDL2

FCRL1

FCRL6
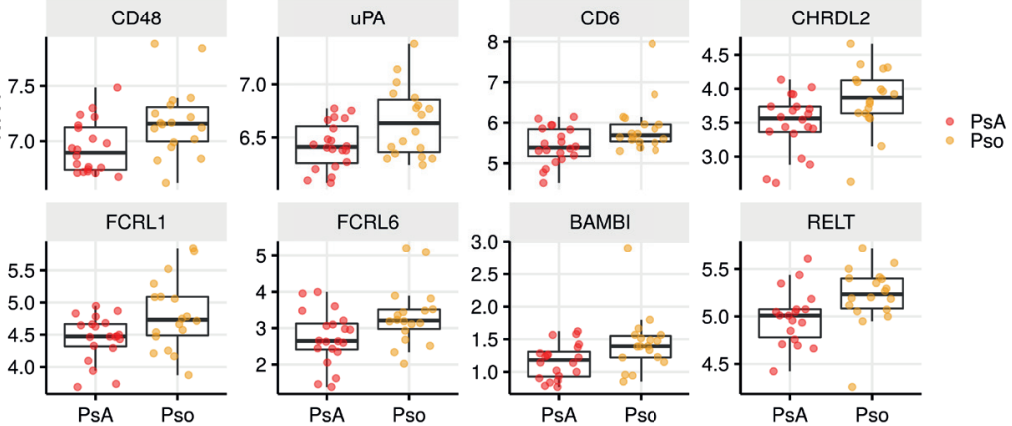

B
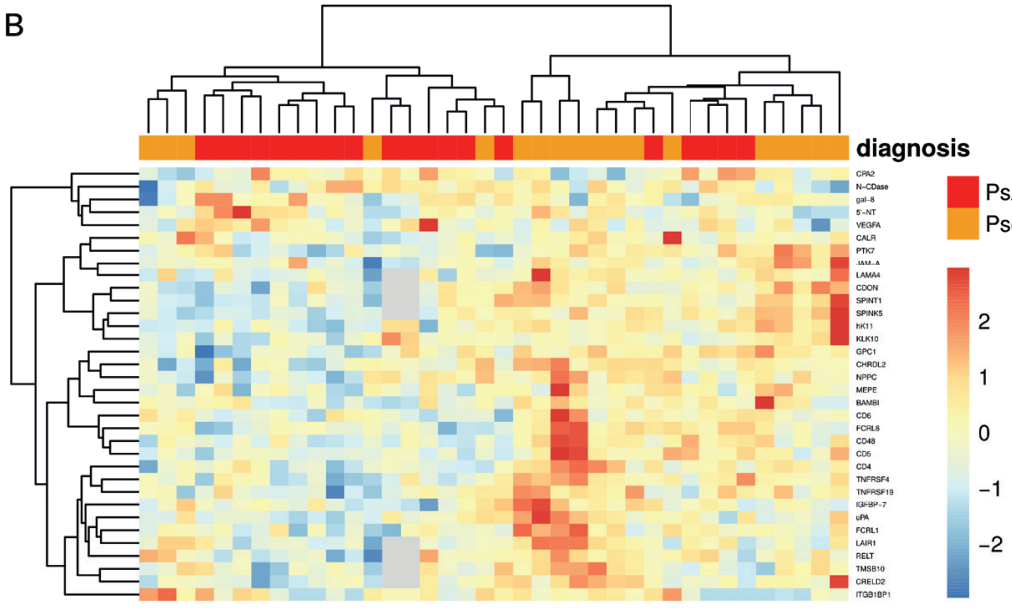

\section{Supplementary Figure S2: Top proteins differentiating PsA from Pso}

2A. Differentially expressed proteins (DEPs) between PsA and Pso. This is an exploratory analysis, showing differentially expressed proteins (DEPs) between PSA and Pso based on nominal P value $<0.05$. The boxplots show the twelve most significant proteins differentiating PSA from HC. 2B. Hierarchical clustering between PsA and Pso. Hierarchical clustering shown in the heatmap shows moderate potential to differentiate PsA from Pso. This analysis is based on DEPs with nominal P value $<0.05$ when comparing PsA to Pso.

Abbreviations: psoriatic arthritis (PsA); Psoriasis (Pso); Normalized Protein expression (NPX); differentially expressed proteins (DEPs); Carboxypeptidase A2 (CPA2); C-type natriuretic peptide (NPPC); Cysteine-rich with EGF-like domain protein 2 (CRELD2); Tumor necrosis factor receptor superfamily member 4 (TNFRSF4 ); CD48 antigen (CD48); Urokinase-type plasminogen activator (UPA); T cell surface glycoprotein CD6 isoform (CD6); Chordin-like protein 2 (CHRDL2); Fc receptor-like protein 1 (FCRL1); Fc receptor-like protein 6 (FCRL6); BMP and activin membrane-bound inhibitor homolog (BAMBI); Tumor necrosis factor receptor superfamily member 19L (RELT) 

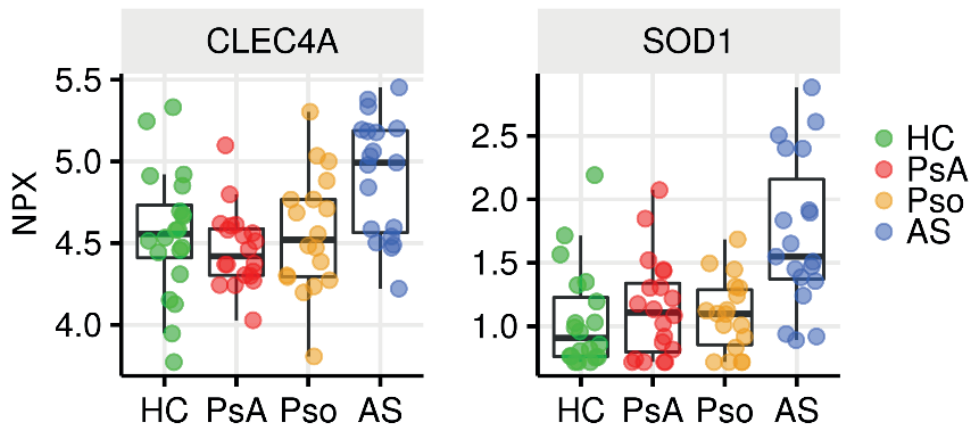

\section{Supplementary Figure S3: CLEC4A and SOD1 elevated in AS.}

CLEC4A was significantly elevated in AS as compared to PSA (FDR-corrected, $p<0.05$ ). SOD1 was significantly elevated in AS as compared to Pso and in AS as compared to HC (FDR-corrected, $\mathrm{p}<0.05$ ).

Abbreviations: healthy control (HC); psoriatic arthritis (PsA); Psoriasis (Pso); Ankylosing spondylitis (AS); Normalized Protein eXpression (NPX); differentially expressed proteins (DEPs); C-type lectin domain family 4 member A (CLEC4A); Superoxide dismutase [Cu-Zn] (SOD1)

A
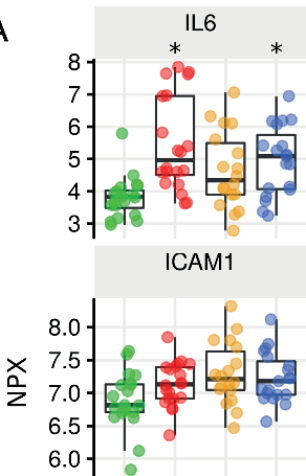

IL13RA1

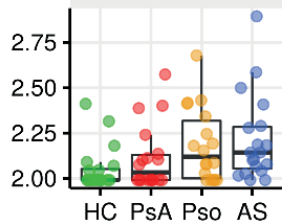

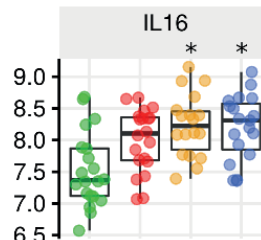

TIE1

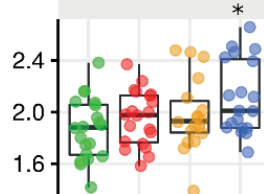

IL-18R1

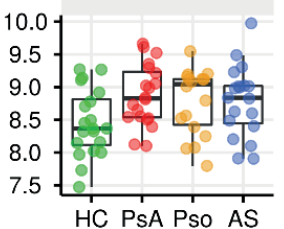

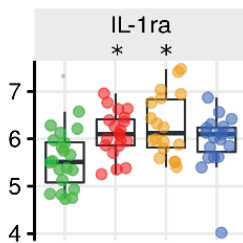

IL-17A

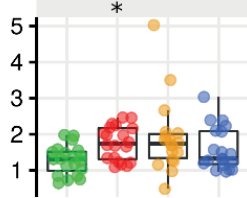

IL-17RA

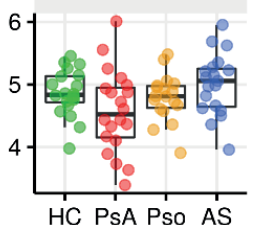

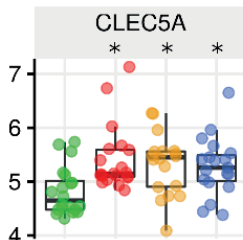

IL-17D

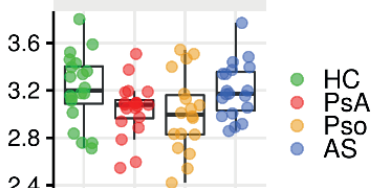

IL13

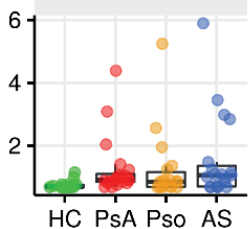


B
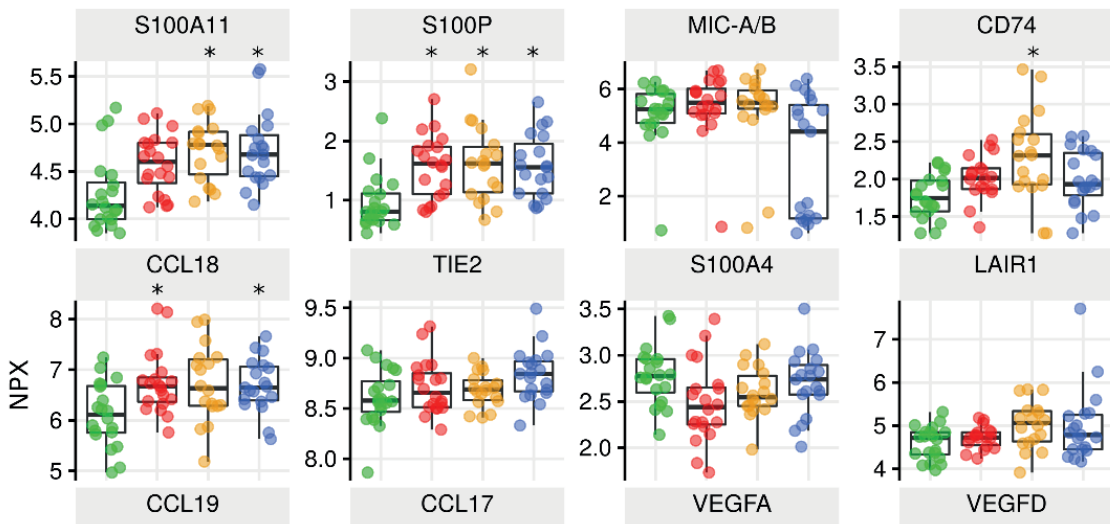

S100A4

LAIR1
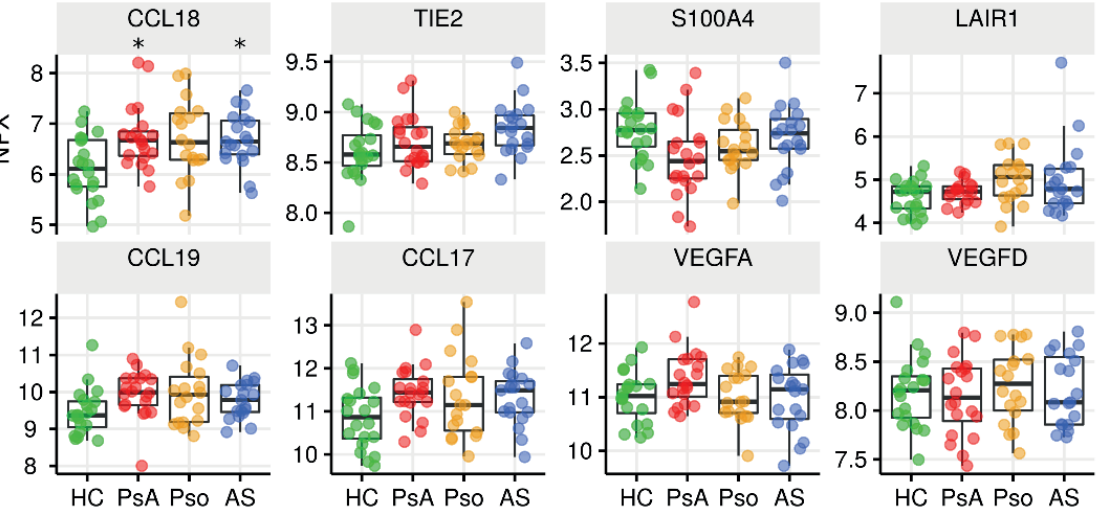

Supplementary Figure S4: Proteins previously implicated in PsA pathogenesis.

A list of literature based proteins of interest is displayed. An asterisk (*) indicates significant difference between $\mathrm{HC}$ and the respective patient group (FDR-corrected, $\mathrm{p}<0.05)$. healthy control $(\mathrm{HC})$; psoriatic arthritis (PsA); Psoriasis (Pso); Ankylosing spondylitis (AS); Normalized Protein eXpression (NPX); differentially expressed proteins (DEPs); Interleukin-6 (IL6); Pro-interleukin-16 (IL16); Interleukin-1 receptor antagonist protein (IL-1ra); C-type lectin domain family 5 member A (CLEC5A); Intercellular adhesion molecule 1 (ICAM1); Tyrosine-protein kinase receptor Tie-1 (TIE1); Interleukin-17A (IL-17A); Interleukin-17D (IL-17D); Interleukin-13 receptor subunit alpha-1 (IL13RA1); Interleukin-18 receptor 1 (IL-18R1); Interleukin-17 receptor A (IL-17RA); Interleukin-13 (IL-13); Protein S100-A11 (S100A11); Protein S100-P (S100P); MHC class I polypeptide-related sequence A/B (MIC-A/B); HLA class II histocompatibility antigen gamma chain (CD74); C-C motif chemokine 18 (CCL18); Angiopoietin-1 receptor (TIE2); Protein S100-A4 (S100A4); Leukocyte-associated immunoglobulin-like receptor 1 (LAIR1); C-C motif chemokine 19 (CCL19); C-C motif chemokine 17 (CCL17); Vascular endothelial growth factor A (VEGF-A); Vascular endothelial growth factor $D$ (VEGFD) 
A

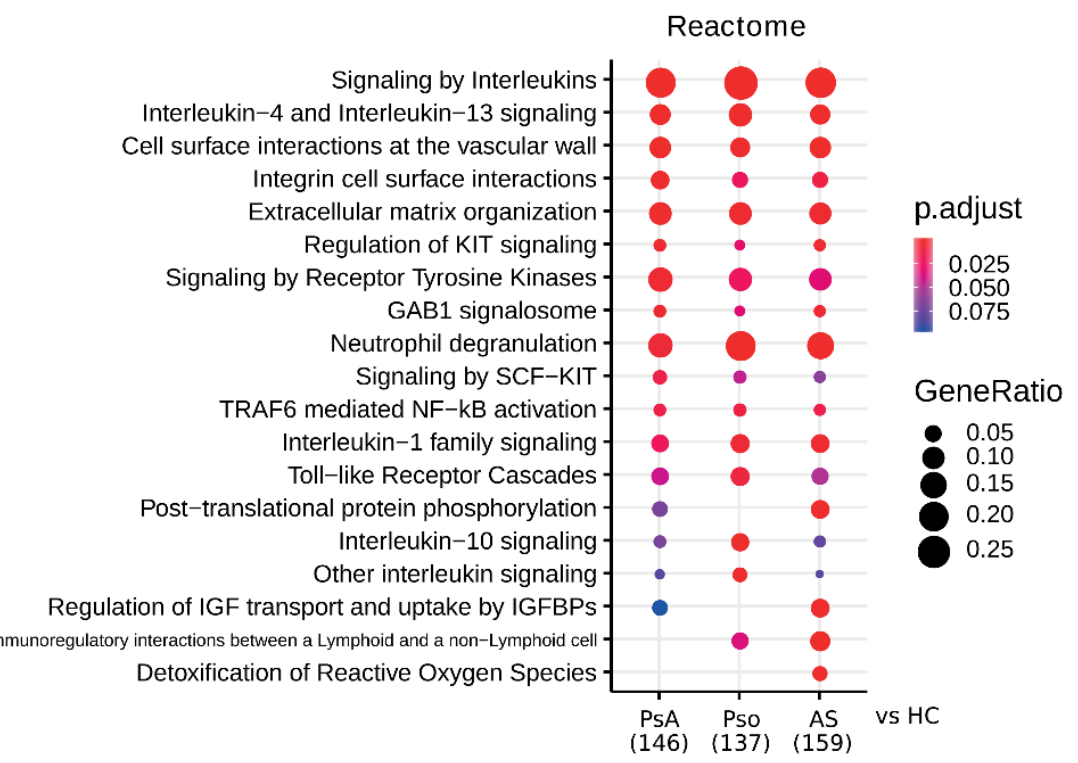

$\mathrm{B}$

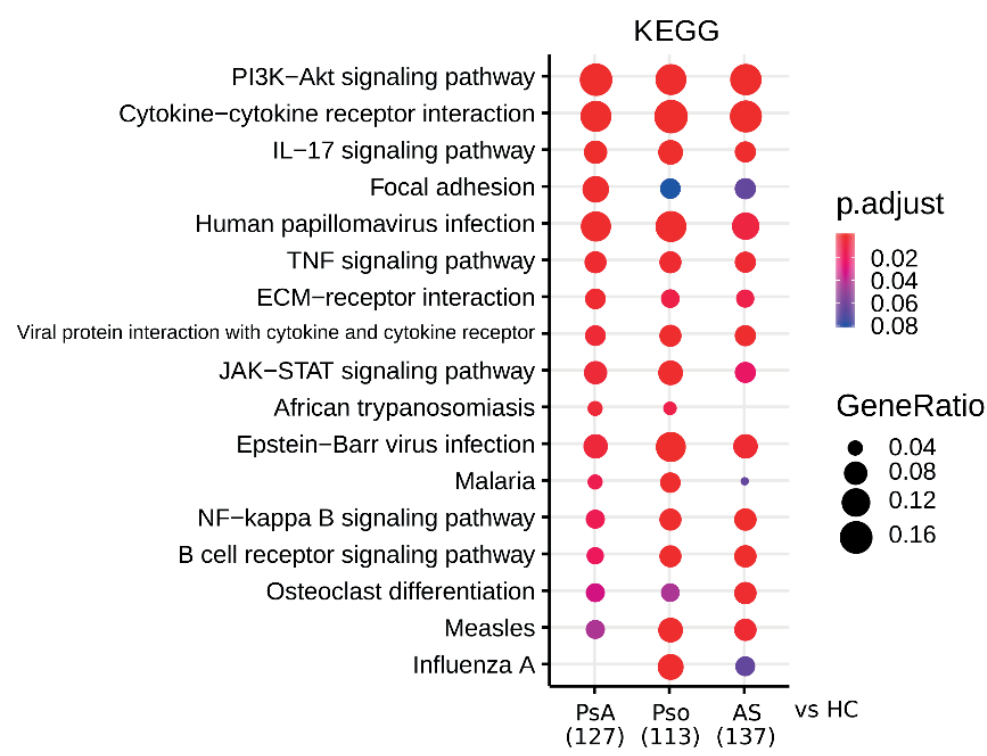

Sup Fig 5: Pso and PsA overlap based on pathway enrichment analysis.

Pathway enrichment analysis was performed based on the differentially expressed proteins (DEPs) with nominal $\mathrm{P}$ value $<0.05$ when comparing $\mathrm{HC}$ versus each patient groups.

Abbreviations: healthy control (HC); psoriatic arthritis (PsA); Psoriasis (Pso); Ankylosing spondylitis (AS); Kyoto Encyclopedia of Genes and Genomes (KEGG); 


\section{Supplementary Tables}

Supplementary Table S1: List of the 68 differentially expressed proteins when comparing PsA to HC.

\begin{tabular}{|c|c|c|c|c|c|}
\hline Olink ID & Protein & groupmean.HC & groupmean.PsA & $\log 2 \mathrm{FC}$ & $P$ value \\
\hline P04083 & ANXA1 & 4.293 & 5.823 & 0.440 & $3.70 \mathrm{E}-06$ \\
\hline O75077 & ADAM 23 & 4.893 & 4.233 & -0.209 & $1.64 \mathrm{E}-05$ \\
\hline P08670 & VIM & 6.314 & 7.257 & 0.201 & $3.22 \mathrm{E}-05$ \\
\hline P19878 & NCF2 & 7.323 & 8.821 & 0.268 & $1.91 \mathrm{E}-04$ \\
\hline Q02880 & TOP2B & 3.806 & 5.062 & 0.411 & 3.81E-04 \\
\hline Q9Y6K9 & NEMO & 5.608 & 6.643 & 0.244 & $4.38 \mathrm{E}-04$ \\
\hline P05231 & IL6 & 3.807 & 5.460 & 0.520 & $4.38 \mathrm{E}-04$ \\
\hline P09769 & FGR & 2.833 & 4.038 & 0.511 & 4.65E-04 \\
\hline P40222 & TXLNA & 4.307 & 5.142 & 0.256 & 4.65E-04 \\
\hline Q6UXH9 & PAMR1 & 5.604 & 6.048 & 0.110 & $6.42 \mathrm{E}-04$ \\
\hline P14317 & HCLS1 & 4.351 & 5.406 & 0.313 & $1.31 \mathrm{E}-03$ \\
\hline Q9GZT9 & EGLN1 & 2.521 & 3.634 & 0.528 & $1.80 \mathrm{E}-03$ \\
\hline P50749 & RASSF2 & 2.770 & 3.981 & 0.523 & 2.37E-03 \\
\hline P78362 & SRPK2 & 0.163 & 0.773 & 2.246 & 2.37E-03 \\
\hline O95544 & NADK & 5.290 & 6.379 & 0.270 & 2.37E-03 \\
\hline 000221 & NFKBIE & 1.990 & 2.584 & 0.376 & $2.40 \mathrm{E}-03$ \\
\hline Q6ZUJ8 & PIK3AP1 & 3.414 & 4.456 & 0.384 & $2.56 \mathrm{E}-03$ \\
\hline P16278 & GLB1 & 3.578 & 2.902 & -0.302 & 2.57E-03 \\
\hline Q7Z5R6 & APBB1IP & 4.147 & 4.459 & 0.105 & $2.90 \mathrm{E}-03$ \\
\hline 014867 & $\mathrm{BACH} 1$ & 1.311 & 2.060 & 0.651 & $3.01 \mathrm{E}-03$ \\
\hline O75475 & PSIP1 & 2.842 & 3.738 & 0.395 & 3.03E-03 \\
\hline Q01543 & FLI1 & 1.312 & 2.119 & 0.692 & $3.21 \mathrm{E}-03$ \\
\hline Q9UM47 & Notch 3 & 5.705 & 5.346 & -0.094 & $3.52 \mathrm{E}-03$ \\
\hline P27695 & APEX1 & 2.094 & 3.134 & 0.582 & $3.54 \mathrm{E}-03$ \\
\hline Q9NX58 & LYAR & 0.264 & 0.849 & 1.684 & 3.55E-03 \\
\hline P25815 & $\mathrm{S} 100 \mathrm{P}$ & 0.954 & 1.561 & 0.711 & $3.70 \mathrm{E}-03$ \\
\hline P42785 & PRCP & 1.881 & 2.237 & 0.250 & $6.31 \mathrm{E}-03$ \\
\hline P55103 & INHBC & 3.727 & 4.294 & 0.204 & $6.31 \mathrm{E}-03$ \\
\hline P49023 & PXN & 3.712 & 4.650 & 0.325 & $6.96 \mathrm{E}-03$ \\
\hline Q9H5Y7 & SLITRK6 & 0.880 & 0.705 & -0.319 & $8.26 \mathrm{E}-03$ \\
\hline Q9NWQ8 & PAG1 & 1.826 & 2.362 & 0.371 & $8.26 \mathrm{E}-03$ \\
\hline Q9HAN9 & NMNAT1 & 5.797 & 6.869 & 0.245 & $8.28 \mathrm{E}-03$ \\
\hline O75569 & PRKRA & 1.031 & 1.523 & 0.562 & $8.74 \mathrm{E}-03$ \\
\hline 060934 & NBN & 3.092 & 4.012 & 0.376 & 9.05E-03 \\
\hline
\end{tabular}




\begin{tabular}{|c|c|c|c|c|c|}
\hline Olink ID & Protein & groupmean.HC & groupmean.PsA & $\log 2 \mathrm{FC}$ & P value \\
\hline Q9UBG3 & CRNN & 5.582 & 4.820 & -0.212 & $9.05 \mathrm{E}-03$ \\
\hline P80511 & EN-RAGE & 5.541 & 6.540 & 0.239 & $9.25 \mathrm{E}-03$ \\
\hline P18510 & IL-1ra & 5.531 & 6.108 & 0.143 & $9.41 \mathrm{E}-03$ \\
\hline Q9NY25 & CLEC5A & 4.818 & 5.409 & 0.167 & $9.50 \mathrm{E}-03$ \\
\hline P16870 & CPE & 6.145 & 5.693 & -0.110 & $1.03 \mathrm{E}-02$ \\
\hline P05089 & ARG1 & 1.620 & 2.454 & 0.599 & $1.05 \mathrm{E}-02$ \\
\hline P07948 & LYN & 2.812 & 3.069 & 0.126 & $1.09 \mathrm{E}-02$ \\
\hline P22466 & GAL & 6.654 & 5.867 & -0.182 & $1.22 \mathrm{E}-02$ \\
\hline Q16552 & IL-17A & 1.286 & 1.756 & 0.450 & 1.40E-02 \\
\hline P09958 & FURIN & 10.576 & 11.001 & 0.057 & $1.69 \mathrm{E}-02$ \\
\hline Q12860 & CNTN1 & 5.318 & 5.007 & -0.087 & $1.72 \mathrm{E}-02$ \\
\hline P06681 & C2 & 5.631 & 5.994 & 0.090 & $1.84 \mathrm{E}-02$ \\
\hline P50452 & SERPINB8 & 2.955 & 3.512 & 0.249 & $1.84 \mathrm{E}-02$ \\
\hline P41236 & PPP1R2 & 2.523 & 3.017 & 0.258 & $1.90 \mathrm{E}-02$ \\
\hline Q92844 & TANK & 1.212 & 1.874 & 0.629 & 1.93E-02 \\
\hline A6NI73 & LILRA5 & 4.470 & 4.793 & 0.100 & $2.10 \mathrm{E}-02$ \\
\hline Q96GP6 & SCARF2 & 6.776 & 6.486 & -0.063 & $2.13 \mathrm{E}-02$ \\
\hline P22681 & CBL & 1.591 & 2.281 & 0.520 & $2.18 \mathrm{E}-02$ \\
\hline Q9BXJ1 & C1QTNF1 & 3.849 & 4.272 & 0.150 & $2.60 \mathrm{E}-02$ \\
\hline P55774 & CCL18 & 6.114 & 6.741 & 0.141 & $2.64 \mathrm{E}-02$ \\
\hline Q9NS68 & TNFRSF19 & 5.204 & 4.881 & -0.093 & $3.16 \mathrm{E}-02$ \\
\hline P11274 & $B C R$ & 2.487 & 3.070 & 0.303 & $3.16 \mathrm{E}-02$ \\
\hline Q99972 & MYOC & 6.212 & 5.695 & -0.125 & $3.20 \mathrm{E}-02$ \\
\hline P63313 & TMSB10 & 2.962 & 3.540 & 0.257 & $3.20 \mathrm{E}-02$ \\
\hline Q13105 & ZBTB17 & 2.547 & 2.952 & 0.213 & $3.20 \mathrm{E}-02$ \\
\hline P24821 & TNC & 2.369 & 2.925 & 0.304 & $3.25 \mathrm{E}-02$ \\
\hline P06756 & ITGAV & 5.405 & 5.222 & -0.050 & $3.25 \mathrm{E}-02$ \\
\hline 000241 & SIRPB1 & 3.887 & 4.279 & 0.139 & $3.37 \mathrm{E}-02$ \\
\hline P08648 & ITGA5 & 3.214 & 3.508 & 0.126 & $3.37 \mathrm{E}-02$ \\
\hline 000273 & DFFA & 3.796 & 4.216 & 0.151 & $3.92 \mathrm{E}-02$ \\
\hline Q6UX15 & LAYN & 5.742 & 5.424 & -0.082 & $3.94 \mathrm{E}-02$ \\
\hline Q12778 & FOXO1 & 1.708 & 2.139 & 0.325 & $3.95 \mathrm{E}-02$ \\
\hline Q16674 & MIA & 11.533 & 11.290 & -0.031 & 4.45E-02 \\
\hline P18564 & ITGB6 & 2.708 & 2.362 & -0.198 & 4.45E-02 \\
\hline
\end{tabular}

$\log 2 \mathrm{FC}=\log _{2}$ fold change. $\mathrm{P}$ value shown is the FDR-corrected $\mathrm{P}$ value. 
Supplementary Table S2: List of the 51 differentially expressed proteins when comparing Pso to HC.

\begin{tabular}{|c|c|c|c|c|c|}
\hline Olink ID & Protein & groupmean.HC & groupmean.Pso & $\log 2 F C$ & P value \\
\hline P04083 & ANXA1 & 4.293 & 5.853 & 0.447 & $8.61 \mathrm{E}-07$ \\
\hline P08670 & VIM & 6.314 & 7.321 & 0.213 & 3.96E-06 \\
\hline P40222 & TXLNA & 4.307 & 5.324 & 0.306 & $3.65 \mathrm{E}-05$ \\
\hline Q9Y6K9 & NEMO & 5.608 & 6.779 & 0.274 & $3.85 \mathrm{E}-05$ \\
\hline Q6ZUJ8 & PIK3AP1 & 3.414 & 4.742 & 0.474 & 3.94E-05 \\
\hline P19878 & NCF2 & 7.323 & 8.985 & 0.295 & $1.34 \mathrm{E}-04$ \\
\hline Q02880 & TOP2B & 3.806 & 5.208 & 0.453 & $1.89 \mathrm{E}-04$ \\
\hline P63313 & TMSB10 & 2.962 & 3.917 & 0.403 & 2.83E-04 \\
\hline P09769 & FGR & 2.833 & 4.001 & 0.498 & 2.83E-04 \\
\hline P07948 & LYN & 2.812 & 3.171 & 0.173 & 4.51E-04 \\
\hline P78362 & SRPK2 & 0.163 & 0.885 & 2.441 & $5.38 \mathrm{E}-04$ \\
\hline P14317 & HCLS1 & 4.351 & 5.415 & 0.315 & 1.19E-03 \\
\hline Q01543 & FLI1 & 1.312 & 2.211 & 0.753 & $1.29 \mathrm{E}-03$ \\
\hline 000221 & NFKBIE & 1.990 & 2.557 & 0.361 & $1.94 \mathrm{E}-03$ \\
\hline P50749 & RASSF2 & 2.770 & 4.083 & 0.560 & $1.94 \mathrm{E}-03$ \\
\hline 075475 & PSIP1 & 2.842 & 3.765 & 0.406 & $2.12 \mathrm{E}-03$ \\
\hline Q9NWQ8 & PAG1 & 1.826 & 2.355 & 0.367 & $2.12 \mathrm{E}-03$ \\
\hline Q92844 & TANK & 1.212 & 2.159 & 0.833 & $2.23 \mathrm{E}-03$ \\
\hline Q12778 & FOX01 & 1.708 & 2.301 & 0.429 & $2.48 \mathrm{E}-03$ \\
\hline 095544 & NADK & 5.290 & 6.422 & 0.280 & $2.51 \mathrm{E}-03$ \\
\hline 014867 & $\mathrm{BACH} 1$ & 1.311 & 2.098 & 0.678 & $2.51 \mathrm{E}-03$ \\
\hline Q14005 & IL16 & 7.529 & 8.236 & 0.129 & $2.84 \mathrm{E}-03$ \\
\hline Q7Z5R6 & APBB1IP & 4.147 & 4.515 & 0.123 & $2.84 \mathrm{E}-03$ \\
\hline Q9GZT9 & EGLN1 & 2.521 & 3.698 & 0.553 & $3.19 \mathrm{E}-03$ \\
\hline P29350 & PTPN6 & 1.262 & 1.679 & 0.411 & 3.37E-03 \\
\hline P31949 & S100A11 & 4.265 & 4.717 & 0.146 & 3.37E-03 \\
\hline P11274 & $\mathrm{BCR}$ & 2.487 & 3.229 & 0.377 & 4.53E-03 \\
\hline P18510 & IL-1 ra & 5.531 & 6.284 & 0.184 & 4.53E-03 \\
\hline Q9NX58 & LYAR & 0.264 & 0.773 & 1.549 & $5.91 \mathrm{E}-03$ \\
\hline P25815 & S100P & 0.954 & 1.620 & 0.764 & $6.18 \mathrm{E}-03$ \\
\hline P01275 & GCG & 4.337 & 3.107 & -0.481 & $6.32 \mathrm{E}-03$ \\
\hline P49023 & PXN & 3.712 & 4.741 & 0.353 & $6.32 \mathrm{E}-03$ \\
\hline P41236 & PPP1R2 & 2.523 & 3.160 & 0.325 & $7.48 \mathrm{E}-03$ \\
\hline P04233 & CD74 & 1.768 & 2.307 & 0.384 & $9.37 \mathrm{E}-03$ \\
\hline P04637 & TP53 & 1.746 & 2.056 & 0.236 & 9.37E-03 \\
\hline
\end{tabular}




\begin{tabular}{llllll}
\hline Olink ID & Protein & groupmean.HC & groupmean.Pso & log2FC & P value \\
\hline P08648 & ITGA5 & 3.214 & 3.695 & 0.201 & $1.04 \mathrm{E}-02$ \\
P27695 & APEX1 & 2.094 & 3.141 & 0.585 & $1.05 \mathrm{E}-02$ \\
O60934 & NBN & 3.092 & 4.041 & 0.386 & $1.12 \mathrm{E}-02$ \\
Q8N5S9 & CAMKK1 & 0.015 & 0.356 & 4.614 & $1.71 \mathrm{E}-02$ \\
Q9HAN9 & NMNAT1 & 5.797 & 6.888 & 0.249 & $1.73 \mathrm{E}-02$ \\
P22466 & GAL & 6.654 & 5.792 & -0.200 & $1.73 \mathrm{E}-02$ \\
O75569 & PRKRA & 1.031 & 1.536 & 0.575 & $1.75 \mathrm{E}-02$ \\
P55103 & INHBC & 3.727 & 4.260 & 0.193 & $1.94 \mathrm{E}-02$ \\
O00241 & SIRPB1 & 3.887 & 4.412 & 0.183 & $1.94 \mathrm{E}-02$ \\
Q9NQ88 & TIGAR & 1.474 & 1.890 & 0.358 & $2.71 \mathrm{E}-02$ \\
P22681 & CBL & 1.591 & 2.292 & 0.527 & $3.63 \mathrm{E}-02$ \\
Q9NY25 & CLEC5A & 4.818 & 5.323 & 0.144 & $4.21 \mathrm{E}-02$ \\
Q00978 & IRF9 & 2.083 & 2.521 & 0.276 & $4.63 \mathrm{E}-02$ \\
P80511 & EN-RAGE & 5.541 & 6.410 & 0.210 & $4.63 \mathrm{E}-02$ \\
P42785 & PRCP & 1.881 & 2.200 & 0.226 & $4.63 \mathrm{E}-02$ \\
P78380 & LOX-1 & 9.070 & 9.601 & 0.082 & $4.76 \mathrm{E}-02$ \\
\hline
\end{tabular}

$\log 2 F C=\log _{2}$ fold change. $P$ value shown is the FDR-corrected $P$ value. 
Supplementary Table S3 List of the 62 differentially expressed proteins when comparing AS to HC.

\begin{tabular}{|c|c|c|c|c|c|}
\hline Olink ID & Protein & groupmean.HC & groupmean.AS & $\log 2 \mathrm{FC}$ & P value \\
\hline Q02880 & TOP2B & 3.806 & 5.518 & 0.536 & $6.66 \mathrm{E}-07$ \\
\hline P78362 & SRPK2 & 0.163 & 0.995 & 2.609 & 4.75E-06 \\
\hline Q9Y6K9 & NEMO & 5.608 & 6.850 & 0.288 & 4.94E-06 \\
\hline P42785 & PRCP & 1.881 & 2.637 & 0.488 & 5.75E-06 \\
\hline P04083 & ANXA1 & 4.293 & 5.677 & 0.403 & $8.23 E-06$ \\
\hline Q6ZUJ8 & PIK3AP1 & 3.414 & 4.709 & 0.464 & 8.96E-06 \\
\hline 014867 & $\mathrm{BACH} 1$ & 1.311 & 2.318 & 0.822 & 1.07E-05 \\
\hline Q92844 & TANK & 1.212 & 2.319 & 0.936 & $1.08 \mathrm{E}-05$ \\
\hline P08670 & VIM & 6.314 & 7.227 & 0.195 & $3.34 \mathrm{E}-05$ \\
\hline P40222 & TXLNA & 4.307 & 5.185 & 0.268 & 1.77E-04 \\
\hline 000221 & NFKBIE & 1.990 & 2.604 & 0.388 & $2.25 \mathrm{E}-04$ \\
\hline P09769 & FGR & 2.833 & 4.038 & 0.511 & $2.81 \mathrm{E}-04$ \\
\hline Q9GZT9 & EGLN1 & 2.521 & 3.802 & 0.593 & 2.81E-04 \\
\hline 075569 & PRKRA & 1.031 & 1.676 & 0.701 & 3.11E-04 \\
\hline 075475 & PSIP1 & 2.842 & 3.901 & 0.457 & 3.11E-04 \\
\hline Q12778 & FOX01 & 1.708 & 2.381 & 0.479 & 3.16E-04 \\
\hline P27695 & APEX1 & 2.094 & 3.305 & 0.659 & 4.20E-04 \\
\hline Q9NX58 & LYAR & 0.264 & 0.922 & 1.803 & 4.35E-04 \\
\hline Q01543 & FLI1 & 1.312 & 2.295 & 0.807 & $6.00 \mathrm{E}-04$ \\
\hline P19878 & NCF2 & 7.323 & 8.804 & 0.266 & 7.83E-04 \\
\hline P14317 & HCLS1 & 4.351 & 5.398 & 0.311 & $9.84 \mathrm{E}-04$ \\
\hline P50749 & RASSF2 & 2.770 & 4.060 & 0.552 & $9.84 \mathrm{E}-04$ \\
\hline P00441 & SOD1 & 1.050 & 1.727 & 0.717 & 1.20E-03 \\
\hline 095544 & NADK & 5.290 & 6.481 & 0.293 & $1.40 \mathrm{E}-03$ \\
\hline 060934 & NBN & 3.092 & 4.175 & 0.433 & 1.60E-03 \\
\hline P05231 & IL6 & 3.807 & 4.948 & 0.378 & $1.74 \mathrm{E}-03$ \\
\hline P80511 & EN-RAGE & 5.541 & 6.800 & 0.295 & 1.95E-03 \\
\hline P08648 & ITGA5 & 3.214 & 3.600 & 0.163 & $2.28 \mathrm{E}-03$ \\
\hline Q9NWQ8 & PAG1 & 1.826 & 2.361 & 0.371 & $2.89 \mathrm{E}-03$ \\
\hline P14543 & NID1 & 4.491 & 4.913 & 0.130 & $2.89 \mathrm{E}-03$ \\
\hline Q96SB3 & PPP1R9B & 2.954 & 3.721 & 0.333 & 2.89E-03 \\
\hline P25815 & S100P & 0.954 & 1.571 & 0.720 & 2.89E-03 \\
\hline P49023 & PXN & 3.712 & 4.752 & 0.356 & 3.42E-03 \\
\hline P11274 & $\mathrm{BCR}$ & 2.487 & 3.215 & 0.370 & 3.78E-03 \\
\hline P05067 & APP & 4.349 & 4.963 & 0.190 & 4.44E-03 \\
\hline
\end{tabular}




\begin{tabular}{llllll}
\hline Olink ID & Protein & groupmean.HC & groupmean.AS & log2FC & P value \\
\hline Q14005 & IL16 & 7.529 & 8.197 & 0.123 & $4.49 \mathrm{E}-03$ \\
P31949 & S100A11 & 4.265 & 4.726 & 0.148 & $4.78 \mathrm{E}-03$ \\
P06681 & C2 & 5.631 & 5.992 & 0.090 & $4.93 \mathrm{E}-03$ \\
P51693 & APLP1 & 6.241 & 6.934 & 0.152 & $4.93 \mathrm{E}-03$ \\
P41236 & PPP1R2 & 2.523 & 3.160 & 0.325 & $5.24 \mathrm{E}-03$ \\
Q9NWZ3 & IRAK4 & 0.826 & 1.428 & 0.791 & $6.12 \mathrm{E}-03$ \\
Q9C0C4 & SEMA4C & 1.238 & 1.454 & 0.232 & $8.08 \mathrm{E}-03$ \\
A6NI73 & LILRA5 & 4.470 & 4.957 & 0.149 & $8.08 \mathrm{E}-03$ \\
Q92765 & SFRP-3 & 2.651 & 3.238 & 0.288 & $8.08 \mathrm{E}-03$ \\
P21217 & FUT3/FUT5 & 3.249 & 3.689 & 0.183 & $8.44 \mathrm{E}-03$ \\
Q05084 & ICA1 & 1.417 & 1.907 & 0.428 & $8.90 \mathrm{E}-03$ \\
P10586 & PTPRF & 4.692 & 5.054 & 0.107 & $1.10 \mathrm{E}-02$ \\
Q07065 & CKAP4 & 5.060 & 5.383 & 0.089 & $1.19 \mathrm{E}-02$ \\
Q9HAN9 & NMNAT1 & 5.797 & 6.901 & 0.252 & $1.43 \mathrm{E}-02$ \\
O00273 & DFFA & 3.796 & 4.329 & 0.189 & $1.56 \mathrm{E}-02$ \\
Q8N423 & LILRB2 & 3.527 & 3.910 & 0.149 & $1.65 \mathrm{E}-02$ \\
P04179 & SOD2 & 9.964 & 10.154 & 0.027 & $2.22 \mathrm{E}-02$ \\
P05089 & ARG1 & 1.620 & 2.460 & 0.603 & $2.44 \mathrm{E}-02$ \\
P63313 & TMSB10 & 2.962 & 3.586 & 0.276 & $2.91 \mathrm{E}-02$ \\
P05543 & SERPINA7 & 4.643 & 5.073 & 0.128 & $3.34 \mathrm{E}-02$ \\
P55774 & CCL18 & 6.114 & 6.689 & 0.130 & $3.65 \mathrm{E}-02$ \\
P07948 & LYN & 2.812 & 3.056 & 0.120 & $4.04 \mathrm{E}-02$ \\
Q16769 & QPCT & 2.632 & 2.997 & 0.187 & $4.31 \mathrm{E}-02$ \\
P19320 & VCAM1 & 4.817 & 5.191 & 0.108 & $4.39 \mathrm{E}-02$ \\
Q6UXH9 & PAMR1 & 5.604 & 5.922 & 0.079 & $4.80 \mathrm{E}-02$ \\
P35590 & TIE1 & 1.868 & 2.117 & 0.181 & $4.81 \mathrm{E}-02$ \\
Q9NY25 & CLEC5A & 4.818 & 5.282 & 0.133 & $4.91 \mathrm{E}-02$ \\
\hline & & & &
\end{tabular}

$\log 2 F C=\log _{2}$ fold change. $P$ value shown is the FDR-corrected $P$ value. 
Supplementary Table S4 List of the $\mathbf{3 4}$ differentially expressed proteins when comparing PsA to Pso.

\begin{tabular}{|c|c|c|c|c|c|}
\hline Olink ID & Protein & groupmean.PsA & groupmean.Pso & $\log 2 F C$ & P value \\
\hline P48052 & CPA2 & 10.949 & 10.507 & -0.059 & $2.51 \mathrm{E}-03$ \\
\hline P23582 & NPPC & 3.824 & 4.370 & 0.193 & $4.22 \mathrm{E}-03$ \\
\hline Q6UXH1 & CRELD2 & 3.639 & 3.980 & 0.129 & 7.21E-03 \\
\hline P43489 & TNFRSF4 & 4.755 & 5.135 & 0.111 & 7.47E-03 \\
\hline P09326 & CD48 & 6.943 & 7.180 & 0.049 & $8.55 \mathrm{E}-03$ \\
\hline P00749 & UPA & 6.425 & 6.652 & 0.050 & 1.16E-02 \\
\hline Q8WWJ7 & CD6 & 5.430 & 5.869 & 0.112 & $1.21 \mathrm{E}-02$ \\
\hline Q6WN34 & CHRDL2 & 3.487 & 3.848 & 0.142 & $1.31 \mathrm{E}-02$ \\
\hline Q96LA6 & FCRL1 & 4.447 & 4.816 & 0.115 & $1.34 \mathrm{E}-02$ \\
\hline Q6DN72 & FCRL6 & 2.730 & 3.342 & 0.292 & $1.56 \mathrm{E}-02$ \\
\hline Q13145 & BAMBI & 1.161 & 1.435 & 0.306 & $2.01 \mathrm{E}-02$ \\
\hline Q969Z4 & RELT & 4.990 & 5.219 & 0.065 & $2.40 \mathrm{E}-02$ \\
\hline Q9NR71 & N-CDase & 5.126 & 4.610 & -0.153 & $2.48 \mathrm{E}-02$ \\
\hline 043278 & SPINT1 & 2.629 & 2.868 & 0.125 & 2.64E-02 \\
\hline P06127 & CD5 & 4.668 & 4.930 & 0.079 & 2.77E-02 \\
\hline Q9Y624 & JAM-A & 6.207 & 6.510 & 0.069 & 3.37E-02 \\
\hline P01730 & CD4 & 5.888 & 6.071 & 0.044 & $3.46 \mathrm{E}-02$ \\
\hline Q9NS68 & TNFRSF19 & 4.881 & 5.130 & 0.072 & $3.55 \mathrm{E}-02$ \\
\hline Q6GTX8 & LAIR1 & 4.722 & 5.013 & 0.086 & $3.81 \mathrm{E}-02$ \\
\hline Q13308 & PTK7 & 1.912 & 2.132 & 0.157 & $3.89 \mathrm{E}-02$ \\
\hline O00214 & gal-8 & 8.220 & 7.904 & -0.057 & $4.01 \mathrm{E}-02$ \\
\hline Q4KMG0 & CDON & 4.470 & 4.741 & 0.085 & 4.05E-02 \\
\hline Q16363 & LAMA4 & 4.434 & 4.660 & 0.072 & $4.22 \mathrm{E}-02$ \\
\hline P15692 & VEGFA & 11.354 & 11.023 & -0.043 & 4.25E-02 \\
\hline P21589 & 5'-NT & 11.868 & 11.547 & -0.040 & 4.36E-02 \\
\hline P63313 & TMSB10 & 3.540 & 3.917 & 0.146 & 4.45E-02 \\
\hline P27797 & CALR & 0.198 & 0.393 & 0.990 & 4.57E-02 \\
\hline Q16270 & IGFBP-7 & 8.473 & 8.686 & 0.036 & 4.63E-02 \\
\hline Q9NQ76 & MEPE & 6.704 & 7.021 & 0.067 & 4.64E-02 \\
\hline 014713 & ITGB1BP1 & 0.121 & 0.236 & 0.963 & $4.66 \mathrm{E}-02$ \\
\hline Q9NQ38 & SPINK5 & 1.318 & 1.551 & 0.235 & $4.68 \mathrm{E}-02$ \\
\hline P35052 & GPC1 & 6.115 & 6.306 & 0.044 & 4.94E-02 \\
\hline 043240 & KLK10 & 3.473 & 3.932 & 0.179 & 4.97E-02 \\
\hline Q9UBX7 & hK11 & 7.677 & 7.981 & 0.056 & 4.98E-02 \\
\hline
\end{tabular}

Analysis based on nominal $\mathrm{P}$ value $<0.05 . \log 2 \mathrm{FC}=\log 2$ fold change. $\mathrm{P}$ value shown is the nominal $\mathrm{P}$ value. 


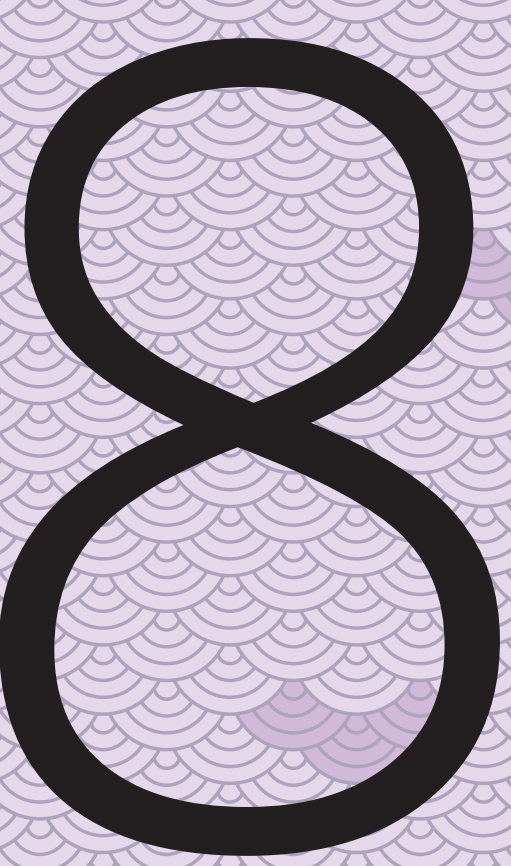




\section{CHAPTER 8}

\section{Limits of traditional evidence-based medicine methodologies exemplified by the novel era in psoriatic arthritis drug development}

Authors:

E.F. Leijten ${ }^{1,2}$, T.R. Radstake ${ }^{1,2}$, I.B. McInnes ${ }^{3}$, J.W. Jacobs ${ }^{1}$

Affiliations

1. Department of Rheumatology and Clinical Immunology, University Medical Center Utrecht, Utrecht, The Netherlands

2. Laboratory of Translational Immunology, University Medical Center Utrecht, Utrecht, The Netherlands

3. Institute of Infection, Immunity and Inflammation, College of Medical, Veterinary and Life Sciences, University of Glasgow, Glasgow, UK

Published in:

Expert Review of Clinical Immunology. 2019 May; 15(5):441-444. doi: 10.1080/1744666X.2019.1580144 


\section{Treating psoriatic arthritis today: the elephant in the room}

An array of new and expensive drugs recently entered the market for psoriatic arthritis (PsA), and several soon will follow, joining the already rich armamentarium(1,2). As a consequence, clinicians will be left with an elephant in the room-'this psoriatic arthritis patient has failed to adequately respond to conventional DMARDs, so which drug (class) should I now prescribe or add?'. Currently, not a single novel drug (biological or targeted synthetic DMARD) has been proven in a head-to-head randomized controlled trial (RCT) superior or inferior to TNF-inhibitors for treating arthritis in patients with PSA.

We argue here that the current method of performing and reporting trial data probably may not be able to answer the aforementioned simple, yet clinically important question. Understanding the caveats of current trials is necessary if we want to improve future clinical decision-making in diseases for which many drug options are available $(3,4)$. For the sake of critical appraisal, here the arthritis 'domain' is primarily discussed and the role of conventional DMARDs is left aside; while also applicable to the whole spondyloarthritis group and other rheumatic diseases, PsA particularly exemplifies the issues raised.

\section{Too many drugs for head-to-head trials}

This first issue originates from the traditional evidence-based medicine approach that seeks head-to-head RCTs to support clinical decisions. In ankylosing spondylitis (AS), for example, both anti-IL-17A and anti-TNF therapies are efficacious and headto-head studies are underway. Although novel drug classes are being tested for AS, at present for AS only two pivotal biological drug classes require comparison(5). And psoriatic skin disease appears to respond well to some biologicals and less so to others, which makes it easier to interpret RCT data and make the choice of the best drug for a patient with predominant cutaneous manifestations (2). For PsA, head-to-head trials are more challenging as we probably will have ten (or more) new drugs soon approved by regulatory agencies (Figure 1)(1). Performing only one or two head-to-head trials (some are underway) is insufficient to provide guidance for clinical decision making given this growing set of options. The design of a single, all-informative '10-drug' head-to-head trial would be overwhelmed by methodological and practical issues. For instance, a massive patient population would need to be included, leveraged on multinational collaborations notwithstanding potential conflicting (pharmaceutical) party interests.

One approach to address the issue above is to indirectly compare the efficacy of drugs from different RCTs using methods that are based on the reported group data, such as network meta-analyses(6). However, it would be more informative 
to analyse individual participant data in such a meta-analysis, thus accounting for the spectrum of responses to the drug from the different trials(7). It is common practice for biological data to be shared upon publication, but publicly sharing raw clinical trial data is unfortunately rare even though this data could provide lowcost insight into optimal treatment strategies for individual patients(8). Another important method of indirectly comparing multiple drugs can be accomplished by setting up large registers, which have the benefit of being able to capture longterm safety data and increase external validity when it comes to efficacy in daily clinical practice(9-11).

\section{Current targets}

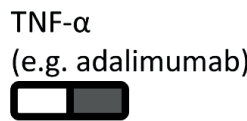

IL-17A

(e.g. secukinumab)

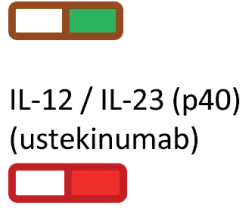

JAK-1 / JAK-3

(tofacitinib)

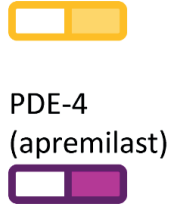

CD80/86

(abatacept)

\section{Emerging targets}

Bispecific cytokine
inhibitors

IL-17F ; IL-17C ;

IL-17 receptor

Research
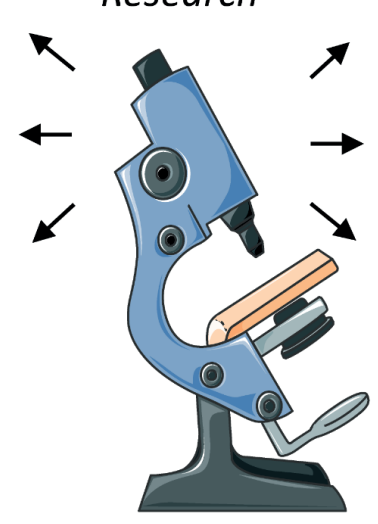

JAK-1

IL-23 (p19) ;

IL-23 receptor

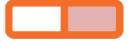

JAK-1

TYK2

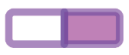

???

Figure 1. Current and emerging drug targets in PsA. Overview of the current therapeutic targets and emerging drug targets for treating arthritis in PSA upon failure to conventional DMARD(s). PDE-4: phosphodiesterase 4, JAK: Janus kinase, TYK2: tyrosine kinase 2. 


\section{Small group differences between active treatments}

Secondly, RCTs are typically powered to identify group differences between placebo and the experimental treatment arms. Biological and other novel drug trials typically yield results of $20-30 \%$ more patients achieving ACR20 response compared to the placebo $\operatorname{arm}(1,2,12)$. Differences in effectiveness between two active treatment arms would be smaller, especially for the outcome arthritis. The typical result from an RCT is displayed by an example in Figure 2; similar data will continue to appear as more drugs enter the market. To detect smaller group differences with respect to improvement in arthritis, the numbers of included patients in the treatment arms need to be much bigger. Indeed, current RCTs with active comparator arms are not powered to detect differences between active treatment arms $(12,13)$. Then again, would small differences in group efficacy data lead to an actual treatment preference in clinical practice and/or (inter)national treatment recommendations? As always, improvements in efficacy will need to be weighed against other factors, such as (long-term) safety, tolerability and cost. Future trials should always compare novel treatments at least to the existing, standard of care treatment ('active comparator') to provide an indication of the added clinical relevance of the novel drug(4). Furthermore, experts should achieve consensus on one specific standard of care drug recommended to be taken along in all future RCTs. This would allow trial data to be reported also as difference versus the 'established' active comparator and greatly enhance RCT data comparisons. 


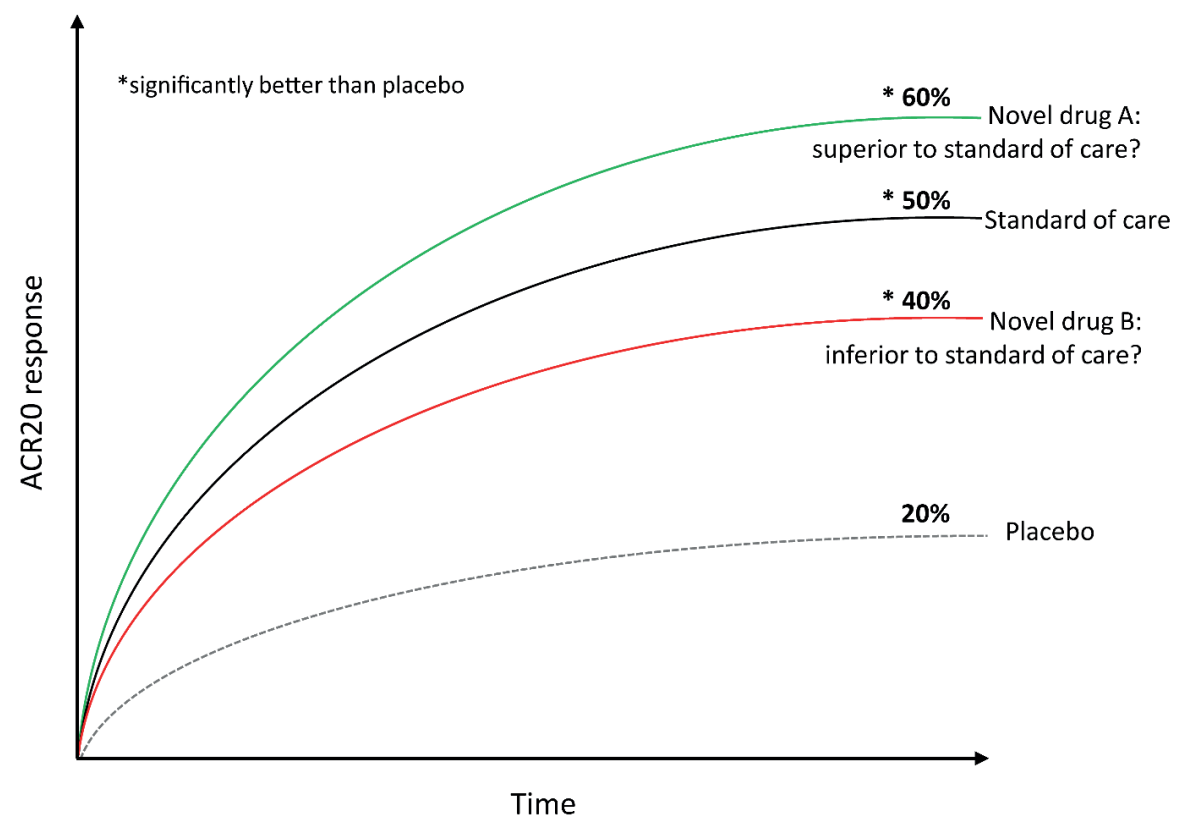

Figure 2. Typical results from a four-arm placebo-controlled RCT. Which drug to prescribe to a patient with active PSA not responding to the current DMARD? For an answer, we look at a (fictitious) RCT with rather sophisticated design and typical results. Probably it will display that all active treatment arms do better than placebo with small differences in efficacy between the active treatment arms. To ascertain whether the differences between active treatment arms are statistically significant, many patients should have been recruited for this trial. Furthermore, this trial only gives answers on the group level, it cannot answer the question for which specific patients novel drug A or novel drug B would have beneficial effects. 


\section{Shortage of strategic trials and issues regarding heterogeneity and outcome measures}

Lastly, future RCTs in PsA with random allocation to different drugs, each with a distinct mode of action, will not inform us about which patient would respond to any drug ('overlapping drug response'), which patient would respond to one specific drug ('selective drug response'), or which patient would fail to respond to any of the drugs (Figure 3$)(3,4)$. We currently include individuals meeting certain classification criteria in RCTs, yet these individuals may not share the specific aberration of molecular mechanisms being targeted by different drugs, and PsA might be a heterogeneous disease. Most patients included in current trials have polyarticular disease, hindering generalisation of study results to patients with mono/oligoarticular disease or manifestations other than arthritis. Rather than lumping all PsA patients into a single trial, future trials should consider enrolling different subsets of PsA patients (e.g. predominant axial disease) with the goal of specifically treating that main manifestation (e.g. outcome measure focussed on axial disease). Probably, with smaller numbers of patients, relevant trial results can be obtained. Such trial-design would enable more personalized choices of therapeutics to be made in daily practice(14).

Currently, most RCTs on PSA use ACR20 response as their primary outcomeborrowed from RA but never validated for PSA-and consensus on a new primary outcome for RCTs in PSA has been slow to form(15). PsA is a disease with marked clinical heterogeneity which contributes to the challenge of choosing a single, overarching outcome measure for all future PsA trials. Recent advanced high-throughput and multi-dimensional analyses have shown that molecular 'fingerprints' / circulating biomarkers have a potential to predict treatment responses for both psoriasis and arthritis (16-18). A great challenge however will be to prove that treatment-algorithms based on those molecular fingerprints are superior to standard of care (i.e. trial-and-error approaches)(19). Oncology can provide methodological guidance; oncologists now often choose drugs based on the individual patients' molecular signature (20-22). Considering the diversity of treatment options targeting different aspects of the disease, PsA is ideally suited to be leader in the rheumatic field when it comes to embracing novel trial designs. In summary, we should allocate time, effort and expertise to modify conventional trial design with the goal to improve the treatment strategy for patients with PsA and other diseases, for which many drug options are available. Coming to terms with the limitations of our current approach of evidence-based medicine for these diseases is the first step in this process. 
Scenario 1: Overlapping drug response

not responding to any of the drugs

responding to novel drug $\mathrm{A}$

responding to standard of care

responding to novel drug $B$

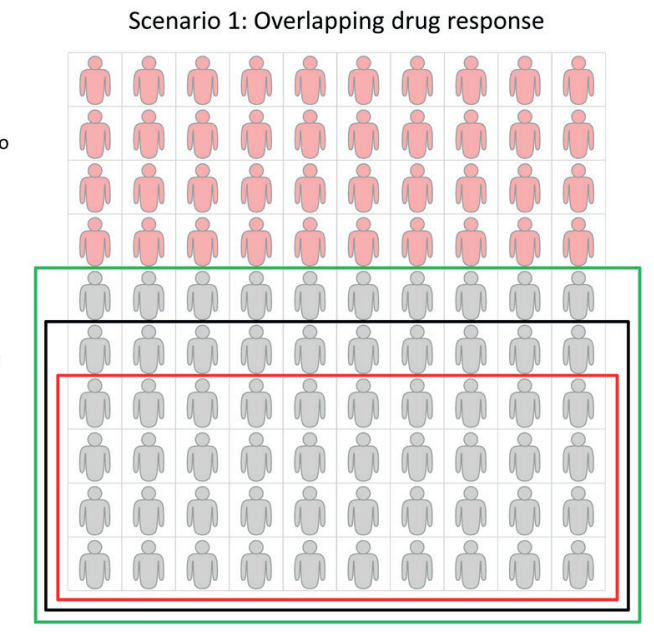

Scenario 2: Selective drug response

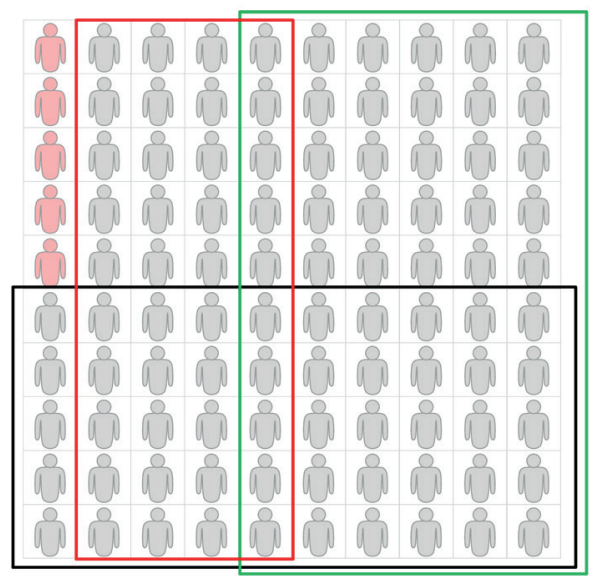

Figure 3. Hypothetical scenarios of drug response. Based on the fictitious RCT results from Figure 2 we illustrate two possible scenarios. Left: The first scenario is that an incremental increase in patients responded to the drugs, mimicking the group efficacy data. All patients who responded to treatment with novel drug B (red area) also responded to standard of care (black area) and novel drug A (green area). This scenario is based on the assumption of 'overlapping drug response' in which patients are either adequately responsive or non-responsive to most drugs. If this scenario is reality, based strictly on efficacy, there would be no place for the standard of care drug nor for novel drug B, as all patients should be treated with novel drug A. Right: The second scenario depicts 'selective drug response', i.e. that each drug targeting a distinct disease pathway elicits a differential drug response in different individuals, based on heterogeneity of and within the disease. If this second scenario is reality, then (1) novel drug B is the most effective treatment for a subgroup of patients, regardless of possible inferior efficacy at the group level and (2) the number of patients who do not respond to any of the three drugs is smaller, compared to in the first scenario. 


\section{References}

1. Elalouf O, Chandran V. Novel Therapeutics in Psoriatic Arthritis. What Is in the Pipeline? Curr Rheumatol Rep. 2018 May;20(7):36.

2. Furue $K$, Ito $T$, Furue M. Differential efficacy of biologic treatments targeting the TNF-a/IL-23/ IL-17 axis in psoriasis and psoriatic arthritis. Cytokine. 2018 Aug;111:182-8.

3. Woodcock J, LaVange LM. Master Protocols to Study Multiple Therapies, Multiple Diseases, or Both. N Engl J Med. 2017;377(1):62-70.

4. Ogdie A, Coates L. The Changing Face of Clinical Trials in Psoriatic Arthritis. Curr Rheumatol Rep. 2017;19(4):21.

5. Poddubnyy $\mathrm{D}$, Sieper J. What is the best treatment target in axial spondyloarthritis: tumour necrosis factor a, interleukin 17, or both? Rheumatology (Oxford). 2017 Oct;

6. Kawalec P, Holko P, Moćko P, Pilc A. Comparative effectiveness of abatacept, apremilast, secukinumab and ustekinumab treatment of psoriatic arthritis: a systematic review and network meta-analysis. Rheumatol Int. 2017;0(0):0.

7. Nash P, Mclnnes IB, Mease PJ, Thom H, Hunger M, Karabis A, et al. Secukinumab Versus Adalimumab for Psoriatic Arthritis: Comparative Effectiveness up to 48 Weeks Using a Matching-Adjusted Indirect Comparison. Rheumatol Ther. 2018 Jun;5(1):99-122.

8. Ohmann C, Banzi R, Canham S, Battaglia S, Matei M, Ariyo C, et al. Sharing and reuse of individual participant data from clinical trials: principles and recommendations. BMJ Open. 2017;7(12):e018647.

9. Frisell T, Dehlin M, Di Giuseppe D, Feltelius N, Turesson C, Askling J, et al. Comparative effectiveness of abatacept, rituximab, tocilizumab and TNFi biologics in RA: results from the nationwide Swedish register. Rheumatology (Oxford). 2019 Jan;

10. Nikiphorou E, Buch MH, Hyrich KL. Biologics registers in RA: methodological aspects, current role and future applications. Nat Rev Rheumatol. 2017 Aug;13(8):503-10.

11. Gladman DD, Coates LC, Jadon DR, Tillett W, Mease PJ, Vis M. The Benefits and Challenges of Setting Up a Longitudinal Psoriatic Arthritis Database. J Rheumatol Suppl. 2018 Jun;94:26-9.

12. Mease P, Hall S, FitzGerald O, van der Heijde D, Merola JF, Avila-Zapata F, et al. Tofacitinib or Adalimumab versus Placebo for Psoriatic Arthritis. N Engl J Med. 2017;377(16):1537-50.

13. Mease PJ, van der Heijde D, Ritchlin CT, Okada M, Cuchacovich RS, Shuler CL, et al. Ixekizumab, an interleukin-17A specific monoclonal antibody, for the treatment of biologic-naive patients with active psoriatic arthritis: results from the 24-week randomised, double-blind, placebocontrolled and active (adalimumab)-controlled period of t. Ann Rheum Dis. 2017 Jan;76(1): $79-87$.

14. Lubrano E, Perrotta FM. Psoriatic arthritis: is it time to treat-to-target or target to treat? Clin Rheumatol. 2017 Dec;36(12):2633-5.

15. Smolen JS, Schöls M, Braun J, Dougados M, FitzGerald O, Gladman DD, et al. Treating axial spondyloarthritis and peripheral spondyloarthritis, especially psoriatic arthritis, to target: 2017 update of recommendations by an international task force. Ann Rheum Dis. 2018;77(1):3-17. 
16. Kim J, Tomalin L, Lee J, Fitz LJ, Berstein G, Correa-da Rosa J, et al. Reduction of Inflammatory and Cardiovascular Proteins in the Blood of Patients with Psoriasis: Differential Responses between Tofacitinib and Etanercept after 4 Weeks of Treatment. J Invest Dermatol. 2018 Feb;138(2):273-81.

17. Miyagawa I, Nakayamada S, Nakano K, Kubo S, Iwata S, Miyazaki Y, et al. Precision medicine using different biological DMARDs based on characteristic phenotypes of peripheral Thelper cells in psoriatic arthritis. Rheumatology (Oxford). 2018 Apr;

18. Teitsma XM, Jacobs JWG, Mokry M, Borm MEA, Pethö-Schramm A, van Laar JM, et al. Identification of differential co-expressed gene networks in early rheumatoid arthritis achieving sustained drug-free remission after treatment with a tocilizumab-based or methotrexate-based strategy. Arthritis Res Ther. 2017 Jul;19(1):170.

19. Leijten EFA. A precision medicine trial in psoriatic arthritis-first jump in the deep end. Rheumatology (Oxford). 2019 Feb 1;58(2):189-90.

20. Herbst RS, Gandara DR, Hirsch FR, Redman MW, LeBlanc M, Mack PC, et al. Lung Master Protocol (Lung-MAP) - A biomarker-driven protocol for accelerating development of therapies for squamous cell lung cancer: SWOG S1400. Clin Cancer Res. 2015;21(7):1514-24.

21. Beckman RA, Antonijevic Z, Kalamegham R, Chen C. Adaptive Design for a Confirmatory Basket Trial in Multiple Tumor Types Based on a Putative Predictive Biomarker. Clin Pharmacol Ther. 2016;100(6):617-25.

22. Hainsworth JD, Meric-Bernstam F, Swanton C, Hurwitz H, Spigel DR, Sweeney C, et al. Targeted Therapy for Advanced Solid Tumors on the Basis of Molecular Profiles: Results From MyPathway, an Open-Label, Phase Ila Multiple Basket Study. J Clin Oncol. 2018 Feb;36(6):536-42. 


$$
9
$$




\section{CHAPTER 9}

General Discussion 
The distinction between psoriasis and psoriatic arthritis (PsA) is the main theme of this thesis. Towards the better identification of the current and future clinical challenges, I have structured the discussion in two parts; in Part 1 of the discussion I revisit specific aspects of the psoriatic disease pathogenesis and place insights from our work and recent work from others in the light of the existing literature. In Part 2 of the discussion I tackle broader questions and put forward overarching premises, again based on work from this thesis, including the pathophysiological insights gained. I conclude each section with questions identified in this thesis that future research endeavors should focus on.

\section{Part 1: Revisiting the pathogenesis of the psoriatic spectrum.}

A main goal of this thesis was to examine specific immunological drivers found in patients with psoriatic arthritis (PsA) as compared to psoriasis without PsA. From a methodological perspective we took the cleanest possible approach: by excluding patients using immunomodulatory drugs that could confound our results and by comparing PSA to psoriasis patients in which concomitant PsA was explicitly excluded. This approach meant we dichotomized the patients: psoriasis versus psoriatic arthritis. The presence or absence of scientific classification criteria determined this dichotomy, with the aim of uncovering unique pathways found in those patients that have concomitant rheumatic disease.

A conceptual overview of the pathogenesis of psoriasis and psoriatic arthritis is portrayed in Figure 1 (page 187). This concept is derived from both randomized controlled-trials studies, but also from studies that examine tissues specifically, and as always does not encompass the full spectrum of all cell-types involved in the pathogenesis. I will here highlight the current concept of the disease pathogenesis to include specific aspects of the work presented in the thesis and show how these data are positioned within the broader context of the disease model.

\section{A. Genetic and environmental background}

It is well-established that both psoriasis and PsA share the majority of genetic predisposition. The strongest genetic association is that of HLA C*0602, found in both Psoriasis and PsA(1). Other MHC-1 molecules or variants are associated with PSA: HLA-A*0201 is associated with PSA, HLA-B27 is specifically associated with PsA (particularly axial disease)(2,3). In addition, specific amino acid variants in the HLA-B allele have been associated with PsA(4). Outside of the MHC-1 region, major genetic susceptibility loci are shared between Psoriasis and PSA, including variants in IL23R, ERAP1, IL12B, TRAF3IP2, TYK2 and NOS2(4,5). However, similar to specific 
variants predisposing to PSA in the MHC-I region, there are non-MHC-I region variants found specifically in PSA: IL23R and TNFAIP3 and PTPN22 and NFKBIA associated with PsA $(4,6,7)$. Also, there is a single-nucleotide polymorphism at chromosome 5q31 associated with PsA. Interestingly, PsA specific risk variants that were identified may serve as epigenetic markers in $\mathrm{CD}^{+}$memory $\mathrm{T}$ cells, thereby providing a theoretical background for the role of CD8 T cells in the disease. However, it is important to remember that genetic susceptibility identified by genome-wide association studies does not necessarily translate directly to gene expression data, while moreover expression level might also be cell-specific and stimulation-dependent(8).

Both cutaneous and rheumatic manifestations can be triggered by local tissue damage, known as the Koebner phenomenon and the deep Koebner phenomenon, respectively(9-12). Clinical cases in which PsA or psoriasis are triggered by damage enforced this idea $(10,13)$ and repeated mechanical stress in an arthritisprone murine model also support this concept $(14,15)$. The microbiome was also implicated as potential environmental contributor, as skin and gut dysbiosis were observed in patients with psoriasis and PsA $(16,17)$. The skin microbiome can also alter local resident $T$ cell function(18) and the skin microbiome is skewed in psoriasis, potentially altering secondary immune cell composition/function including the induction of IL-17A, IL-17F and IL-22 and GATA3 transcripts(17). The interaction between different microbes and resident immune cells in the skin is complex and far from being elucidated, but has the capacity to modify innate and adaptive immune responses(19). Perhaps other environmental factors, such as dietary components, indirectly impact immune cell homeostasis(20). In summary, psoriasis and PsA share a genetic background that provide environmental triggers to incite an aberrant immune response (Figure 1, box A).

\section{B. Primary cutaneous events}

Overall, both the adaptive and innate immune response contribute to the pathogenesis of psoriasis(10,21). As depicted in the figure, major phenotypic, transcriptomic and functional factors are conserved across multiple adaptive and innate lymphocytes $(22,23)$. In the skin the pathogenic events are thought to first arise from damage, upon which keratinocytes release antimicrobial peptides (AMPs), including $\mathbf{S 1 0 0}$ proteins, B-defensin and cathelicidin(24). Specifically anti-microbial peptides such as cathelicidins (i.e., LL-37) has been found to form complexes with self-DNA, which can activate the innate immune response by triggering Toll-Like Receptors on plasmacytoid dendritic cells that thereby release type 1 interferon (IFN)(25,26). As a consequence of expression of such an induced powerful IFN signature, which is characteristic for Psoriasis as well as PsA, the stage is set for dendritic cells to mature and skew $\mathrm{T}$ cell response to type 1 or 
type 17 cell polarization $(27,28)$. During the development of psoriatic plaques, the presence of the IFN signature coincides with an influx of pathogenic T cells $(24,26)$. $T$ cells have historically been deemed critical immune cells in the pathogenesis of psoriasis, a concept that was fueled by their marked influx in the skin, and the knowledge that T-cell directed therapy (ciclosporine) is an effective treatment. Indeed, their importance is still re-affirmed by recent studies(29-33). Interest in the IFN signature associated IL-23-IL-17 pathway has soared over recent years, which could overshadow the original point of view that psoriasis is driven by T-helper 1 cells (Th1) $(21,24)$. Psoriatic skin contains both CD8 and CD4 T cells that produce large amounts of the classically denominated Type 1 cytokines: tumor necrosis factor-alpha (TNF-a) and IFN-gamma(34,35).

More recent insights, most notably the discovery of IL-23-IL-17 axis adds a layer of complexity to the original Th1/Th2 balance(32). As subsequently will be discussed, the third type of cells are now clearly established as important mediators of pathogenesis in psoriasis and PsA. For the narrative that follows, I denote the relevant cell types Type 1 or Type 17-the former as predominant IFN-gamma producer and the latter as capable of producing IL-17 (Figure 1, box B). There are of course exceptions and nuances to this conceptual simplification, which will be explained at a later stage.

Many of effector functions that at earlier stage were attributed to T cells of the adaptive immune system, are now also known to be fulfilled by an array of recently discovered innate-type lymphocytes (Chapter 5)(36). Specifically, an array of lymphocytes lacking the classical alpha-beta T-cell receptors capable of producing IL-17 have been and continue to be discovered(22,37,38). For example, in murine models of psoriasis, gamma-delta $T$ cells produce a large fraction of the disease-associated IL-17, although their human counterparts may contribute less to the overall type 17 response $(39,40)$. Other cells are also known to produce IL-17, including mast cells and neutrophils and innate lymphocytes (ILCS). Work by Villanova et al. and Teunissen et al. described their discovery of ILCs in the skin, with skewing toward type 17 response and expressing the natural cytotoxicity receptor NKp44(41,42). More recent investigations into the role of ILCs in skin immune homeostasis also yielded insight that ILCs express RORC-the master transcriptional factor conserved across type 17 cells $(22,43)$.

One question that I have tried to address, is which of the different cellular subset of the type 1 and/or type 17 category is the principle culprit in psoriasis and PsA? The answer is that it is complicated. For example, first dermal T cells and subsequently epidermal T cells start proliferating during human psoriasis development, with a cascade that can be initiated solely by resident T cells in a TNF-a dependent manner (35). Murine models do help in providing mechanistic insight, but clarify 
a part of the immune dysregulation observed rather than the full complexity of the human disease. Different models have identified distinct on/off switches necessary for mimicking psoriatic-like phenotype $(26,36)$

Taken together, the psoriatic immunopathology highlights how a diverse array of immune cells-adaptive and innate-can have convergent, down-stream effects on the tissue and stromal cells in which these cells are positioned.

\section{Primary synovio-entheseal events}

Moving forward from recent knowledge on the pathogenesis of skin, we revisit the pathology in the joint, which encompasses both synovium, entheses, bone and cartilage. The inflammatory trigger is deemed to start at the entheses first, followed by synovitis $(44,45)$. Clearly, from an experimental point of view, the joint is more difficult to study than the skin because the tissue is less accessible, and our knowledge about the local pathogenesis is therefore more limited. Nonetheless, specific studies together did build an overall notion of the local factors that drive the arthritis phenotype. The histopathology of synovitis has been studied in PsA, often using RA as comparison, and consists of synovial lining hyperplasia, neoangiogenesis and immune cell infiltrates including macrophages, neutrophils and lymphocytes(46). Sometimes the synovial fluid and synovial tissue are considered interchangeable "compartments" although specific cellular subsets can be found in the one compartment, but not per se the other compartment. For example: B-cells are not detected in synovial fluid yet are clearly present in the synovial tissue(47).

In PsA, specific anatomic sites prone to high mechanical stress are commonly affected by enthesitis and support the notion that local damage could spark an inflammatory cascade of the enthesis. Different lymphoid, myeloid and stromal cells have been found to be necessary to drive murine models of PsA and/or spondyloarthritis, with a consistent role for the entheses as primary starting point $(14,15,22,48-50)$. However, the driving factors upstream of the type 1 and type 17 cell presence and response is uncertain in the human setting. Myeloid dendritic cells have recently been found in entheseal tissue capable of producing IL-23, potentially driving further development, maturation and/or triggering of type 17 cells. Moreover, these myeloid dendritic cells could produce CCL20, the chemoattract for CCR6 that is typically expressed by type 17 cells(51) (Figure 1, box C).

Similar to the skin, IL-17 production in the synovial fluid and tissue traced back to distinct immune cell subsets including CD4 and CD8 T cells(52-56). Also, entheseal tissue was found to harbour resident $T$ cells that can produce both TNF and IL-17(57). GM-CSF is another chemokine/cytokine likely involved in the local pathogenesis of the disease and can be produced by different cells(58). 
Interestingly, in the synovial tissue and fluid T cells are not easily classified as purely Type 1 or Type 17(47,58,59). Our conventional phenotyping studies on stimulated peripheral blood lymphocytes (Chapter 6) confirm that any dichotomy ("Th17 cells") is an oversimplification, i.e. cells are rarely "sole" producer of one, single cytokine. In support, T cells recently described in synovial tissue are indeed "polyfunctional" clearly surpassing any classical distinction, e.g. type 17 cells co-producing granzymes (!) (55). Perhaps the chronic inflammation in the synovium has skewed these cells. Either way, these findings are in apparent disagreement with the Type 17 CD8 T cells we describe in Chapter 6. However, in agreement with the Type 17 CD8 T cells we describe as derived from skin, recently identified $T$ cells in the entheses also retain a more homogenous pattern: i.e. type 17 cells co-express a more regulatory transcriptional profile, clearly distinct from the Type 1 functionality(57).

The synovial fluid contains numerous cell types, with a predominance for CD8 T cells. Clonal expansion of CD8 T cells has been described in synovial fluid(60). More recently, Penkava et al. detailed TCR sequencing and transcriptomic analysis of synovial tissue and synovial fluid CD8 T cells revealed clonally expanded CD8 T cells, which exhibit high expression of HLA-DR and CXCR3(47). These cytotoxic CD8 T cells expressed high levels of granzymes (simplified as Type 1 cells in Figure 1) are thus in sharp contrast to the CCR10+ CD8 T cells we described in circulation that were traced back to a cutaneous origin (Chapter 6). The CCR10+ CD8 T cells we described have inherent homeostatic function, with Tc17/Tc22-like characteristics and devoid of cytotoxicity markers(61). The cells described by Penkava et al. from the synovial fluid/tissue do closely mimic a recently described cytotoxic, resident CD8 T cell population from epidermal origin (CD49a+) (31). Curiously, this specific CD49a+ CD8 T cells from the epidermis was not found in the psoriasis patients, but rather the vitiligo patients(!). In summary, T cells in the synovial fluid and tissue -as compared to T cells in enthesis and skin-appear to have more overlapping Type 1 and Type 17 functionality.

As compared to the more heterogeneous population of $\mathrm{T}$ cells in the synovial tissue/fluid, the innate lymphocytes retain a cleaner type 1 versus type 17 profile. The discovery of ILC3 in synovial fluid of PSA was of particular interest since ILC3 were not enhanced in RA synovial fluid (Chapter 4 ). These results are striking because the synovial fluid in arthritis results in an "inflammatory soup", at which point it may be hard to decipher which components are primarily responsible for the disease and which components are most likely a secondary effect. Our data underscore possible mechanistic sequelae in PsA whereby a Type 17 response is reflected by innate lymphoid cells, which somewhat offsets the more dominant type 1 response as perpetuated by conventional T cells described above. We were one of the first to 
describe the presence of ILC3 in PSA synovial fluid, which in the meantime has been confirmed by other groups, although the functional profile differs with respect to functional production of IL-22, GM-CSF and/or IL-17(58,62-65).

Considering the scarcity of ILC in terms of absolute number of cells (both in both skin and synovial fluid) an obvious question arises-to what extent do these cells actually contribute to the disease? Similar to the question tackled in the section on cutaneous events, this question is difficult to answer. We suspect their role should be placed in the greater context of the immunologic processes. It has been shown that ILC3-independent of conventional T cells_could induce psoriasis in healthy human skin xenotransplants, at least supporting the notion that these cells are capable of pathogenicity(66). Overall, the role for innate lymphoid cells in PsA is probably most predominant during the early stages of the disease, preceding the adaptive immune response, with an eventual function that convergences with other Type 17 cells at a later stage.

Taken together, these findings underscore (1) that across tissue sites an array of type 1 and type 17 cells with convergent pathways and broad similarities may be detected, but (2) that the individual strength of the type 1 or type 17 profile is not conserved across tissues even within the same disease. Accordingly, the cellular response may diverge across tissues within the same disease.

\section{Secondary effects on local and distant tissues}

Upon convergence of cell-overarching effector functions, the local tissue may respond differently depending on the primary site affected. Taking the skin as example, IL-17A makes keratinocytes respond by abnormal differentiation and hyperproliferation. In addition, it induces keratinocytes to produce CCL20, interleukin 6 (IL-6), antimicrobial peptides (e.g. LL-37, S100A7) and chemokines that attract neutrophils (e.g. CXCL1, CXCL8) [reviewed in (67)]. Neutrophils are typically found in the dermis and can form ("Munro's") micro-abscesses. TNF-a also induces CCL20 production by keratinocytes, which is the chemoattractant for CCR6 (i.e. prototypical Th17 bearing receptor) (68). Instead, IFN-gamma induces keratinocyte production of ICAM1 and CXCL10 (amongst other ligands of the CXCR3 receptor), which prototypically recruit type 1 function on T cells(69). During this secondary phase, the the type 1 and type 17 responses are not mutually exclusive on the local tissue: combined stimulation of TNF and IL-17 have known synergistic effects(70). Furthermore, type 1 interferon and also IFN-gamma (type 2 interferon) can further amplify the IL-23-IL-17 axis(71). Together, the secondary effects may alter the composition and function of local immune cells in the tissue. Clinically-unaffected psoriatic skin harbors a modified gene transcriptional signature-which includes elevated transcripts of IL-22, type 1 interferon, CXCL10, 
IL-17 and different AMPs(72). Cytokines are important in driving immune cells from unaffected psoriatic skin to incite an aberrant response(35) and it has been shown that after psoriasis is clinically resolved, the local skin still contains a resident population of T cells which can produce IL-17A(30,73). In summary, the type 1 and type 3 response can induce a synergistic, self-amplifying feedback loop on the local tissue and local immune cells (Figure 1 box D).

However, can the local tissue factors also cause "cross-tissue contamination"? Or are the skin and joint disease completely independent of each other? Much less is known about possible secondary effects on distant tissue sites. It is known that the same $T$ cell clones that reside in clinically-resolved psoriasis skin are also detected (to a lesser degree) in non-lesional skin-indicating secondary trafficking to distant sites that have not been phenotypically affected is plausible(73,74). A small population of clonally expanded CD8 T cells has also recently been traced across synovial tissue, synovial fluid and blood compartment(47). Murine models suggest that circulating factors such as cytokines could have an effect on distant tissue sites, raising the question whether primary events occurring at the skin could trigger distant effects in the synovium or entheses. From a methodological perspective the head-to-head comparison of blood samples from patients with PsA and psoriasis (Chapters 6-7) provides insight into the question whether cross-contamination can theoretically occur. Simplistically, if there are messages (cells, soluble factors, auto-antibodies, etc.) being sent to distant sites this must travel via the circulating compartment.

Overall, in our work we noted very large differences in the circulating compartment between psoriasis and PsA as compared to healthy controls. In contrast, the circulating compartment between psoriasis and PsA showed major overlap and only detailed, sometimes even exploratory, analysis was needed to find any difference at all (Chapter 6-7). This is noteworthy not only from a diagnostic/ biomarker perspective, but also from a pathophysiological perspective. In our cohorts, the patients with psoriasis and PsA had similar level of psoriasis skin disease severity, whereas the patients with PsA had concomitant active arthritis. So how to interpret this? Does this mean that the pathology at the synovio-entheseal site itself explains very little variance in the circulating immune compartment? And does this exclude the possibility of cross-tissue contamination?

In the literature there was to date a relatively scarcity of head-to-head studies comparing immune cells or circulating factors in psoriasis to PsA(75-78). Benham et al. found enhanced IL-22 secretion in stimulated PBMCs in PSA(54) and work from the group of Dafna Gladman has consistently reported CXCL10 as being elevated in patients with PSA as compared to psoriasis, even before the onset of PSA development (79-81). Specific auto-antibodies have been discovered in the context of psoriasis, including to LL37, a disintegrin and metalloprotease domain containing 
thrombospondin type 1 motif-like 5 (ADAMTSL5), with higher levels in PsA. However, the pathogenic role of these auto-antibodies remains unknown(82-84).

In our work, one of the key distinguishing features was the finding of an enhanced levels of skin-tropic CD8 T cells in PsA as compared to psoriasis. These cells are inherently tolerogenic and reside in the skin, and we postulate that local factors and genetic background in patients with PsA could induce greater number of these cells and skew them away from a regulatory towards more pro-inflammatory (i.e. Tc17-like) function. We theorize that these cells could either option (i) directly contribute to the pathogenesis via soluble factors that incite local/distant effects or option (ii) rather be seen as a flag/marker for disturbances in skin homeostasis.

In support of option (i): murine models underscore the concept that soluble mediators can be upstream events driving spondyloarthritis phenotype $(49,85)$. Of specific relevance to the concept of cross-tissue contamination, Chen et al recently described a murine model in which keratinocyte-specific overproduction of IL-23 could induce secondary distant effects, resulting in synovial hyperplasia, dactylitis and enthesitis(86). Theoretically soluble factors could therefore migrate to distant tissue sites and modify stromal cell and resident immune cell interaction. However, when we revisit our own proteomic data in circulation they do not support the concept that soluble factors have a strong distinguishing signature between psoriasis and PsA. Clearly some caution should be warranted, because our investigation was cross-sectional which may not reflect subtle, cumulative differences in the soluble factors that may impose a burden over time. The model by Chen et al surprisingly did not detect an enhanced level of IL-23 in the circulation of these mice. Rather, they found a strong upregulation of IL-22 and in a later phase upregulated CXCL10 in the circulation. In support of the aforementioned option (ii): the strong upregulation of IL-22 in the circulation did not itself contribute to driving the pathogenesis, but was rather deemed protective(86,87)(!).

Overall, this is an interesting, and largely unexplored intersection between the skin and joint disease that deserves future investigation. When comparing psoriasis to PsA, both possibilities_direct pathogenic role versus flag/marker for homeostatic disturbance-should be kept in mind. Lastly, it is important that any potential distinguishing features (cell subset, auto-antibodies, soluble factors, etc.) be replicated in independent cohorts of patients. 
In summary, psoriasis and PsA have predominantly shared genetic background and overlapping immunologic signature, confirming their denomination as falling within a spectrum of one single disease $(9,88)$. Local tissue factors induce a primary inflammatory response, followed by a persistent inflammatory environment that hosts innate and adaptive immune cells with overlapping functional effects. The local inflammatory process of the tissue is capable of determining the phenotype: where psoriasis and/or arthritis can occur in an independent fashion both from clinical and pathophysiological point of view. In the circulating compartment we detected only subtle changes between patients with psoriasis compared to PsA, indicating that if cross-tissue contamination occurs, this is a chronic and subtle process not readily detectible by cross-sectional screening. Future work should examine if the inflammatory response at the primary tissue site is capable of priming distant tissue sites for a secondary inflammatory response, in order to better understand and better treat PsA. 


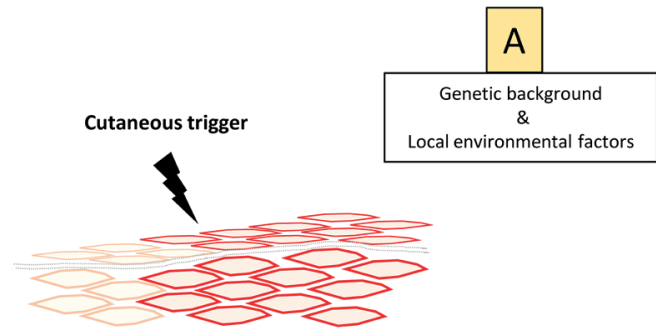

Synovio-entheseal trigger

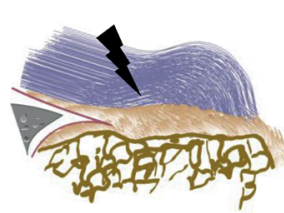

\section{B}
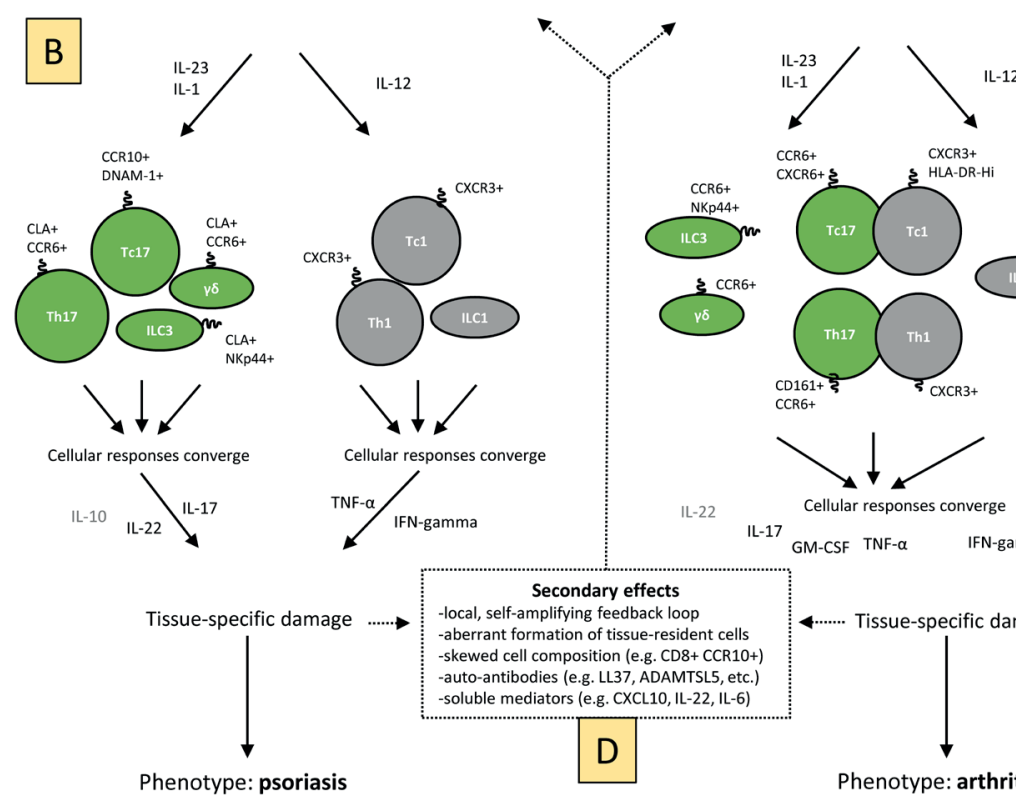

CCR6+

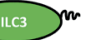

\& CCR6+

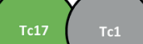

Tc1

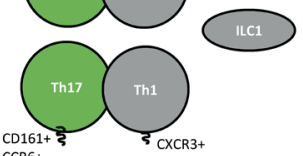

CCR6+

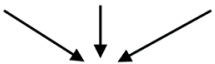

Cellular responses converge Granzymes

GM-CSF TNF- $\alpha \quad$ IFN-gamma

4...... Tissue-specific damage

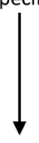

Phenotype: arthritis

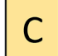

\section{Legend}

Type 1 Type 17

Adaptive $\bigcirc \bigcirc$

Innate 00

Figure 1. Based on the genetic background-largely shared in psoriasis and psoriatic arthritis-local environmental factors and tissue damage induce a primary immune response. This is followed by a persistent inflammatory environment that hosts innate and adaptive immune cells with overlapping functional effects, simplified as Type 1 or Type 17. The figure shows that this pattern is more heterogeneous on the rheumatic disease side. Each local inflammatory environment will be mimicked by a local phenotype, which can occur in an independent fashion of any other tissue site that is affected. The primary tissue site affected can incite (i) again effects on the primary site affected, creating a selfamplifying inflammatory loop and (ii) theoretically, though largely unexplored, have distant effects on secondary tissue sites which we have termed "cross-tissue contamination". 


\section{Part 2: Lessons learned}

\section{How to identify PsA in the larger pool of psoriasis patients?}

PsA is commonly undiagnosed in the larger pool of psoriasis patients $(89,90)$. Our results from Chapter 2 affirm the prevalent clinical description of PsA: that there were no straight-forward clinical parameters that discriminate which patients have PsA in the larger pool of patients with psoriasis. For example, C-reactive protein and erythrocyte sedimentation rate were not elevated in patients with PsA compared to psoriasis. Also, there was no major difference in psoriasis skin disease severity.

Instead, screening for PsA in psoriasis patients attending the dermatology clinics might be assisted by use of the simple psoriasis epidemiology screening tool (PEST) questionnaire. While this method is not a perfect diagnostic tool, it provides the treating dermatologist with a user-friendly tool that can be readily applied in clinical practice. Past and current work suggested that 3 out of 5 questions positive on this PEST questionnaire is deemed the optimal cutoff for identifying PsA(91-93), but we recommend that the threshold be lowered to 2 out of 5 questions. Recent datasets also highlight that the sensitivity is strongly impacted: with a sensitivity of $81 \%$ using the cutoff of 2 versus sensitivity of $58 \%$ using the cutoff of 3(93). Clearly, lowering the threshold (always) reduces specificity while increases sensitivity of the test. We argue that if this screening tool be used in daily practice, the dermatologist would best appreciate a test with high sensitivity, in order to exclude PsA with greater confidence.

An additional consideration is that our test was performed in patients attending the dermatology clinic, whereas implementation of this test (with relatively low specificity) in a psoriasis population with low prevalence of PsA such as the primary care setting, would subsequently result in an unfavorable (costeffective) strategy $(94,95)$. Our work did not address the question whether early screening and identification of PsA results in better outcomes in the long run. Circumstantial evidence indicates late referral is associated with a diminished health outcome(96). At the same time, this does not mean that early referral or early intensified treatment regimens per se improve the outcome. Recent work from the TICOPA trial investigated the treatment using "tight control", i.e. rapidly escalating treatment when the treatment goal had not been attained. This approach did not result in fewer erosions-the gold standard to prove a window of opportunity-but did improve a number of other clinical outcomes $(97,98)$.

All of the available screening questionnaires are aptly described as "moderately accurate" as diagnostic tool(99). We would argue that beyond the "methodologically correct" use of the PEST questionnaire-dictating which patients should and which should not be referred based on the PEST score-the actual implementation of 
this questionnaire into daily practice provides a constant reminder to the treating dermatologist that (1) he or she should be aware of concomitant PsA and (2) that the patient is more than welcome to attend the rheumatologic clinic for an evaluation if PSA is suspected. While these two points may seem trivial, it is of utmost importance to have good collaboration and reciprocal willingness across medical specialties, considering that they often treat the same patient.

Recommendation: screening questionnaires for PsA should be implemented in care for psoriasis patients attending dermatology clinics

Open question: Is screening for PsA cost-effective and will this prevent structural damage (erosions)?

\section{How should we approach the treatment of PsA?}

Why is the aforementioned cross-disciplinary collaboration between dermatologist and rheumatologist so relevant for patients with psoriasis and PsA? Firstly, the dermatological and rheumatological treatment strategies can be remarkably dissimilar, which has both a scientific merit as well as should be seen within historical context (Introduction to this thesis). The scientific merit is based on the fact that certain treatment options are more effective for certain disease manifestations(100). However, in daily clinical practice there is also a strong historical basis for the divergent treatment regimens employed by dermatologist and rheumatologist. The distinction between the rheumatological treatment regimen and the dermatological treatment regimen becomes more apparent by examining the data presented in Chapter 3. For example, sulfasalazine had been prescribed as a first line treatment for patients with PsA in our rheumatology department, whereas this drug is never prescribed by a dermatologist since this has no proven effect on improving psoriatic skin disease. Vice versa, none of the patients from our rheumatology department were treated with fumaric acidwhich is a drug often prescribed by the dermatologist, but has historically never been part of the arsenal of drugs being prescribed by the rheumatologist.

However, over the last years the dermatological versus rheumatological divergence in treatment regimen across specialties has narrowed. Certain medications are now recommended to be preferentially initiated for treating skin manifestations whereas other preferentially initiated for treating rheumatic manifestations. There are major campaigns for awareness-to rheumatologists that psoriasis is an important factor in determining quality of life-and to dermatologists to be aware of concomitant PSA. The domain-based treatment approach has also been adopted by multiple treatment guidelines/recommendations(101-103). 
The data presented in Chapter 3 provide additional evidence for treatment recommendations/guidelines for PsA, to support the choice of methotrexate (particularly in those with relevant skin disease) in patients with PsA suffering arthritis(101-103). The preference for methotrexate over other conventional disease modifying anti-rheumatic drugs is pronouncedly stated in the European League Against Rheumatism guidelines, with the nuance that this is partly based on "consensual expert opinion", and our data from daily clinical practice support this recommendation. Our results indicate that methotrexate had much longer monotherapy drug retention rates than sulfasalazine, which we deemed an indirect parameter for efficacy.

However, in the individual patient with PsA it will be important for dermatologist and rheumatologist to make the best choice of treatment when both psoriasis and arthritis are active-not simply focusing on one domain. An infrastructure is needed to provide rapid and easy cross-talk between dermatologist and rheumatologist, aimed at reciprocal willingness to confer on the best treatment choice and matters of daily practicality (Who prescribes the drug? Who follows the patients? etc.). The two aforementioned points are needed and the implementation of a screening questionnaire provides a solid starting point to streamline treatment of the individual patient.

Recommendation: major phenotypic manifestation should drive the choice of therapy, with methotrexate as preferred option over sulfasalazine for treating arthritis.

Open question: which major biological and non-biological factors contribute to treatment deemed as successful?

\section{Which insights are gained for personalized medicine?}

With advances in our understanding of the disease pathogenesis of PsA, it is tempting to envision precision medicine in the near future, particularly considering the large number of different therapeutics on the market(104). In Chapter $\mathbf{8}$ we critically evaluate trial design in the field and make recommendations for improvements. Moreover, we discuss the concept of a selective drug response, which is an important example of what falls under the term personalized medicine. Considering the array of treatment options, can we predict which drug would be most effective for the individual patient? This questions entails both clinical and biological considerations. 
Firstly, response or non-response to treatment is not solely a biological phenomenon reflected by an outcome measure or disease activity scale(105). An array of factors will eventually determine whether a patient ends up in remission of the disease and whether patient and physician deem the treatment a success(106). Some clear examples of this are seen in Chapter 3: multiple different factors contribute to drug-cessation. One of these was inefficacy, but side-effects were responsible for nearly one-third of "treatment failure" in daily clinical practice. Thus identifying molecular fingerprints that predict side-effects and long-term drug-retention may be equally important as identifying the molecular fingerprint for the reduction of a disease activity measure.

Secondly, there are methodological considerations with respect to the concept of personalized medicine. For the sake of focus, take the search for a molecular fingerprint to predict treatment response (i.e. reduction in disease activity measure). In PsA, a recent study tested the proof of the principle comparing different biological agents, in which specific immune cell subsets predicted better/ worse treatment response $(107,108)$. However, predictive biomarker studies are ideally done on baseline samples embedded within the setting of randomized controlled trials (RCTs). Previous work done in our center indicate that clinical factors are still the main predictors of treatment response in RA(109). Continued pioneering work in the dermatological field_combining the strength of RCTs with high-throughput technologies-indicates that biomarkers can aid in predicting response to different treatment options with respect to psoriasis(110-112), but it remains to be determined if the same holds true for rheumatic manifestations.

Thirdly, there is the biological consideration to take into account: how specific are these drugs in fact? From a black-and-white point of view monoclonal antibodies indeed target completely different molecules. For example, if we take an RCT comparing for example antibodies to IL-17A and TNF-a, perform molecular fingerprinting, then theoretically we are comparing two "modes of action". However, as discussed in the section on the pathogenesis, the distinction between the cells that produce these molecules, the downstream pathways they activate, and the self-amplifying loop often converge $(111,112)$. Rightly so the term "polyfunctional" T cells better captures the function of T cells in PsA $(59,113)$.

In summary, there are clinical and biological considerations to take into account when striving for personalized medicine, which includes the concept that monoclonal antibodies targeting different cytokines should not per se be considered as different "modes of action". 
Does this mean the glass is half empty with respect to precision medicine? No, instead we can apply the knowledge on clinical and pathogenic heterogeneity to optimize future study design. As argued throughout the general discussion: psoriasis and PsA are part of a spectrum of diseases with common pathogenic drivers, which means we need to let go of the categorical approach of studying the disease.

From a clinical point of view, this would mean that a search for predictors of therapy response should first focus on predicting response to the individual disease domain, rather than a multidomain outcome for PsA. For example, it would be more logical to first uncover predictors of response to psoriatic skin disease (PASI), as compared to predictors of complete remission in multiple domains containing psoriasis, enthesitis and arthritis. This follows the principle that local tissue factors primarily determine the phenotype and that common factors shared across tissues may show an increased/decreased dominance per tissue site. In support, paired skin versus synovial analysis found that IL-17 has higher expression in skin, while TNF-a /IFN-gamma are more prominent in synovium(114,115). Moreover, recent transcriptomic analysis of paired skin and synovial tissue also confirms the notion of tissue specific transcriptomic profiles: divulging that some features show overlap across tissue (e.g. TNF-a), while other features do not consistently overlap across tissues (e.g. IL-23)(116). Looking to the future, studies collecting paired tissue samples from multiple sites should be specifically stimulated to help uncover novel treatment avenues-for the treatment response to arthritis still lags far behind the treatment response to psoriasis. Tissue collection remains exceptionally challenging, particularly when it comes to the synovio-entheseal site (which only a limited number of research groups bravely study(117)) and the fact that many of the affected joints (e.g. distal interphalangeal joints) are not readily accessible to study. One of the open questions is if more readily-accessible tissue sites or other cellular models can be studied as proxy for the disease process and/ or treatment response.

Recommendation: main phenotypic manifestations should drive study participant selection for both clinical and basic research questions, without the need to dichotomize PsA versus psoriasis.

Open question: which tissue is the most relevant to study and can more readily approachable tissues serve as biomarker to study disease pathogenesis and treatment response? 


\section{Concluding remarks: clinical necessity and patience}

Research questions should always be driven by clinical necessity. The patient perspective has a key role and there is a continued need for clinicians who treat these patients in daily clinical practice to help translate clinical necessity into more formal research questions / hypothesis. Dialogue between patient, clinician and non-clinical scientist is essential to come to the core of the research questions being addressed. A basic level of understanding each other's "language" is needed for this to be successful. Once the research question is formulated, there is a prominent role for the research team to devise the best strategy of addressing the question. At times it can be deemed that the researcher (fundamental, statistical, bioinformatician, etc.) is "far from the bed", but it is a shared responsibility of the research team to reduce that distance. Examples of doing this have been undertaken in the past in our laboratory: organizing "lab days" for patients and study participants is a good example of how we can bridge this gap in a positive manner. Further endeavors such as presenting basic scientific data to clinicians and to patient-organizations is another way to challenge the research team in a positive manner to translate their findings to a broader public ear. We need to continually strive to optimize such collaboration.

Lastly is the acceptance of time as core criterium for high-quality research. Goals should always be made to attain results at the long-term. This is not dissimilar to the daily clinical practice in treating patients with rheumatic disease-a short course of corticosteroids is rarely the best approach-we need to invest in the long run because, in the end, that will provide the best balance between efficacy versus side-effects. And yes this will sometimes be dissatisfying to the research team, patients, managers and funding-agencies, but accepting time as core criterium for high-quality research will pay off in the end. From our own personal experience we have from scratch written study protocols, recruited patients, performed indepth clinical and biological analysis and have many years later starting to provide meaningful results, with the ultimate goal of further improving patient care. 


\section{References}

1. Okada Y, Han B, Tsoi LC, Stuart PE, Ellinghaus E, Tejasvi T, et al. Fine Mapping Major Histocompatibility Complex Associations in Psoriasis and Its Clinical Subtypes. Am J Hum Genet. 2014 Aug;95(2):162-72.

2. Brown MA, Xu H, Li Z. Genetics and the axial spondyloarthritis spectrum. Rheumatology. 2020 Oct 1;59(Supplement_4):iv58-66.

3. Feld J, Ye JY, Chandran V, Inman RD, Haroon N, Cook R, et al. Is axial psoriatic arthritis distinct from ankylosing spondylitis with and without concomitant psoriasis? Rheumatology. 2020 Jun 1;59(6):1340-6.

4. Bowes J, Budu-Aggrey A, Huffmeier U, Uebe S, Steel K, Hebert HL, et al. Dense genotyping of immune-related susceptibility loci reveals new insights into the genetics of psoriatic arthritis. Nat Commun. 2015 Feb 5;6:6046.

5. Stuart PE, Nair RP, Tsoi LC, Tejasvi T, Das S, Kang HM, et al. Genome-wide Association Analysis of Psoriatic Arthritis and Cutaneous Psoriasis Reveals Differences in Their Genetic Architecture. Am J Hum Genet. 2015;97(6):816-36.

6. Bowes J, Loehr S, Budu-Aggrey A, Uebe S, Bruce IN, Feletar M, et al. PTPN22 is associated with susceptibility to psoriatic arthritis but not psoriasis: evidence for a further PsA-specific risk locus. Ann Rheum Dis. 2015 Oct; 74(10):1882-5.

7. Zhao Q, Sun $Y$, Fu X, Wang Z, Yu G, Yue Z, et al. Identification of a Single Nucleotide Polymorphism in NFKBIA with Different Effects on Psoriatic Arthritis and Cutaneous Psoriasis in China. Acta Derm Venereol. 2018;

8. Shi C, Rattray M, Barton A, Bowes J, Orozco G. Using functional genomics to advance the understanding of psoriatic arthritis. Rheumatology. 2020 Nov 1;59(11):3137-46.

9. Boehncke W-H. Psoriasis and Psoriatic Arthritis: Flip Sides of the Coin? Acta Derm Venereol. 2016 May;96(4):436-41.

10. Boehncke W-H, Schön MP. Psoriasis. Lancet. 2015 Sep;386(9997):983-94.

11. Ritchlin CT, Colbert RA, Gladman DD. Psoriatic Arthritis. N Engl J Med. 2017;376(10):957-70.

12. Veale DJ, Fearon U. The pathogenesis of psoriatic arthritis. Lancet (London, England). 2018;391(10136):2273-84.

13. Hsieh J, Kadavath S, Efthimiou P. Can traumatic injury trigger psoriatic arthritis? A review of the literature. Clin Rheumatol. 2013 Nov 19;

14. Jacques P, Lambrecht S, Verheugen E, Pauwels E, Kollias G, Armaka M, et al. Proof of concept: enthesitis and new bone formation in spondyloarthritis are driven by mechanical strain and stromal cells. Ann Rheum Dis. 2014 Feb;73(2):437-45.

15. Cambré I, Gaublomme D, Burssens A, Jacques P, Schryvers N, De Muynck A, et al. Mechanical strain determines the site-specific localization of inflammation and tissue damage in arthritis. Nat Commun. 2018 Dec 5;9(1):4613.

16. Scher JU, Ubeda C, Artacho A, Attur M, Isaac S, Reddy SM, et al. Decreased Bacterial Diversity Characterizes the Altered Gut Microbiota in Patients With Psoriatic Arthritis, Resembling Dysbiosis in Inflammatory Bowel Disease. Arthritis Rheumatol. 2015 Jan;67(1):128-39. 
17. Chang H-W, Yan D, Singh R, Liu J, Lu X, Ucmak D, et al. Alteration of the cutaneous microbiome in psoriasis and potential role in Th17 polarization. Microbiome. 2018 Dec 5;6(1):154.

18. Naik S, Bouladoux N, Wilhelm C, Molloy MJ, Salcedo R, Kastenmuller W, et al. Compartmentalized Control of Skin Immunity by Resident Commensals. Science (80- ). 2012 Aug 31;337(6098):1115-9.

19. Belkaid $\mathrm{Y}$, Tamoutounour $\mathrm{S}$. The influence of skin microorganisms on cutaneous immunity. Nat Rev Immunol. 2016 Jun 27;16(6):353-66.

20. Shi Z, Wu X, Yu S, Huynh M, Jena PK, Nguyen M, et al. Short-Term Exposure to a Western Diet Induces Psoriasiform Dermatitis by Promoting Accumulation of IL-17A-Producing $\gamma \delta$ T Cells. J Invest Dermatol. 2020 Sep;140(9):1815-23.

21. Lowes M a, Suárez-Fariñas M, Krueger JG. Immunology of psoriasis. Annu Rev Immunol. 2014 Jan;32:227-55.

22. Venken $\mathrm{K}$, Jacques $\mathrm{P}$, Mortier C, Labadia ME, Decruy T, Coudenys J, et al. RORyt inhibition selectively targets IL-17 producing iNKT and $\gamma \delta-T$ cells enriched in Spondyloarthritis patients. Nat Commun. 2019;10(1):9.

23. Gracey E, Hromadová D, Lim M, Qaiyum Z, Zeng M, Yao Y, et al. TYK2 inhibition reduces type 3 immunity and modifies disease progression in murine spondyloarthritis. J Clin Invest. 2020 Mar 9;130(4):1863-78.

24. Nestle FO, Kaplan DH, Barker J. Psoriasis. N Engl J Med. 2009 Jul 30;361(5):496-509.

25. Lande R, Gregorio J, Facchinetti V, Chatterjee B, Wang Y-H, Homey B, et al. Plasmacytoid dendritic cells sense self-DNA coupled with antimicrobial peptide. Nature. 2007 Oct 4;449(7162):564-9.

26. Nestle FO, Conrad C, Tun-Kyi A, Homey B, Gombert M, Boyman O, et al. Plasmacytoid predendritic cells initiate psoriasis through interferon-alpha production. J Exp Med. $2005 \mathrm{Jul}$ 4;202(1):135-43.

27. Mylonas A, Conrad C. Psoriasis: Classical vs. Paradoxical. The Yin-Yang of TNF and Type I Interferon. Front Immunol. 2018 Nov 28;9.

28. Santini SM, Lapenta C, Donati S, Spadaro F, Belardelli F, Ferrantini M. Interferon-a-conditioned human monocytes combine a Th1-orienting attitude with the induction of autologous Th17 responses: role of IL-23 and IL-12. PLoS One. 2011 Jan;6(2):e17364.

29. Stockenhuber K, Hegazy AN, West NR, llott NE, Stockenhuber A, Bullers SJ, et al. Foxp3+ T reg cells control psoriasiform inflammation by restraining an IFN-I-driven CD8+ T cell response. J Exp Med. 2018 Aug 6;215(8):1987-98.

30. Cheuk S, Wikén M, Blomqvist L, Nylén S, Talme T, Ståhle M, et al. Epidermal Th22 and Tc17 Cells Form a Localized Disease Memory in Clinically Healed Psoriasis. J Immunol. 2014 Apr 1;192(7):3111-20.

31. Cheuk S, Schlums H, Gallais Sérézal I, Martini E, Chiang SC, Marquardt N, et al. CD49a Expression Defines Tissue-Resident CD8 + T Cells Poised for Cytotoxic Function in Human Skin. Immunity. 2017 Feb;46(2):287-300.

32. Teunissen MBM, Bos JD, Koomen CW, de Waal Malefyt R, Wierenga EA. Interleukin-17 and Interferon-y Synergize in the Enhancement of Proinflammatory Cytokine Production by Human Keratinocytes. J Invest Dermatol. 1998 Oct;111(4):645-9. 


\section{CHAPTER 9}

33. Lowes MA, Kikuchi T, Fuentes-Duculan J, Cardinale I, Zaba LC, Haider AS, et al. Psoriasis Vulgaris Lesions Contain Discrete Populations of Th1 and Th17 T Cells. J Invest Dermatol. 2008 May;128(5):1207-11.

34. Austin LM, Ozawa M, Kikuchi T, Walters IB, Krueger JG. The Majority of Epidermal T Cells in Psoriasis Vulgaris Lesions can Produce Type 1 Cytokines, Interferon-y, Interleukin-2, and Tumor Necrosis Factor-a, Defining TC1 (Cytotoxic T Lymphocyte) and TH1 Effector Populations:1 a Type 1 Differentiation Bias is a. J Invest Dermatol. 1999 Nov;113(5):752-9.

35. Boyman O, Hefti HP, Conrad C, Nickoloff BJ, Suter M, Nestle FO. Spontaneous Development of Psoriasis in a New Animal Model Shows an Essential Role for Resident T Cells and Tumor Necrosis Factor-a. J Exp Med. 2004 Mar 1;199(5):731-6.

36. Pantelyushin $\mathrm{S}$, Haak S, Ingold B, Kulig P, Heppner FL, Alexander a, et al. psoriasiform plaque formation in mice Brief report Ror $\mathrm{y} t+$ innate lymphocytes and $\mathrm{y} \delta \mathrm{T}$ cells initiate psoriasiform plaque formation in mice. 2012;122(6):2252-6.

37. Keijsers RRMC, Joosten I, van Erp PE., Koenen HJP., van de Kerkhof PCM. Cellular sources of il-17: a paradigm shift? Exp Dermatol. 2014 Jul;n/a-n/a.

38. Sato Y, Ogawa E, Okuyama R. Role of Innate Immune Cells in Psoriasis. Int J Mol Sci. 2020 Sep 9;21(18):6604.

39. Laggner U, Di Meglio P, Perera GK, Hundhausen C, Lacy KE, Ali N, et al. Identification of a Novel Proinflammatory Human Skin-Homing Vy 9 V 2 T Cell Subset with a Potential Role in Psoriasis. J Immunol. 2011 Sep 1;187(5):2783-93.

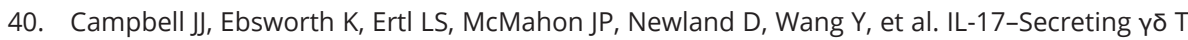
Cells Are Completely Dependent upon CCR6 for Homing to Inflamed Skin. J Immunol. 2017 Nov 1;199(9):3129-36.

41. Villanova F, Flutter B, Tosi I, Grys K, Sreeneebus H, Perera GK, et al. Characterization of innate lymphoid cells in human skin and blood demonstrates increase of NKp44+ ILC3 in psoriasis. J Invest Dermatol. 2014 Apr;134(4):984-91.

42. Teunissen MBM, Munneke JM, Bernink JH, Spuls PI, Res PCM, Te Velde A, et al. Composition of Innate Lymphoid Cell Subsets in the Human Skin: Enrichment of NCR(+) ILC3 in Lesional Skin and Blood of Psoriasis Patients. J Invest Dermatol. 2014;13.

43. Brüggen M-C, Bauer WM, Reininger B, Clim E, Captarencu C, Steiner GE, et al. In Situ Mapping of Innate Lymphoid Cells in Human Skin: Evidence for Remarkable Differences between Normal and Inflamed Skin. J Invest Dermatol. 2016 Dec;136(12):2396-405.

44. McGonagle D, Ash Z, Dickie L, McDermott M, Aydin SZ. The early phase of psoriatic arthritis. Ann Rheum Dis. 2011 Mar;70 Suppl 1(Suppl 1):i71-6.

45. McGonagle D, Gibbon W, Emery P. Classification of inflammatory arthritis by enthesitis. Lancet. 1998 Oct;352(9134):1137-40.

46. Celis R, Cuervo A, Ramírez J, Cañete JD. Psoriatic Synovitis: Singularity and Potential Clinical Implications. Front Med. 2019 Feb 11;6.

47. Penkava F, Velasco-Herrera MDC, Young MD, Yager N, Nwosu LN, Pratt AG, et al. Single-cell sequencing reveals clonal expansions of pro-inflammatory synovial CD8 T cells expressing tissue-homing receptors in psoriatic arthritis. Nat Commun. 2020 Dec 21;11(1):4767. 
48. Frankenberger M, Hofer TPJ, Marei A, Dayyani F, Schewe S, Strasser C, et al. Transcript profiling of CD16-positive monocytes reveals a unique molecular fingerprint. Eur J Immunol. 2012 Apr;42(4):957-74.

49. Reinhardt A, Prinz I. Whodunit? The Contribution of Interleukin (IL)-17/IL-22-Producing $ү \delta ~ T$ Cells, a $\beta$ T Cells, and Innate Lymphoid Cells to the Pathogenesis of Spondyloarthritis. Front Immunol. 2018;9:885.

50. Cuthbert RJ, Watad A, Fragkakis EM, Dunsmuir R, Loughenbury P, Khan A, et al. Evidence that tissue resident human enthesis y $\delta \mathrm{T}$-cells can produce IL-17A independently of IL-23R transcript expression. Ann Rheum Dis. 2019 Nov;78(11):1559-65.

51. Bridgewood C, Watad A, Russell T, Palmer TM, Marzo-Ortega H, Khan A, et al. Identification of myeloid cells in the human enthesis as the main source of local IL-23 production. Ann Rheum Dis. 2019 Jul;78(7):929-33.

52. Menon B, Gullick NJ, Walter GJ, Rajasekhar M, Garrood T, Evans HG, et al. Interleukin$17+C D 8+T$ cells are enriched in the joints of patients with psoriatic arthritis and correlate with disease activity and joint damage progression. Arthritis Rheumatol (Hoboken, NJ). 2014 May;66(5):1272-81.

53. Noordenbos T, Yeremenko N, Gofita I, van de Sande M, Tak PP, Caňete JD, et al. Interleukin-17positive mast cells contribute to synovial inflammation in spondylarthritis. Arthritis Rheum. 2012 Jan;64(1):99-109.

54. Benham H, Norris P, Goodall J, Wechalekar MD, Fitzgerald O, Szentpetery A, et al. Th17 and Th22 cells in psoriatic arthritis and psoriasis. Arthritis Res Ther. 2013 Jan;15(5):R136.

55. Steel KJA, Srenathan U, Ridley M, Durham LE, Wu S-Y, Ryan SE, et al. Polyfunctional, Proinflammatory, Tissue-Resident Memory Phenotype and Function of Synovial Interleukin-17A+CD8+ T Cells in Psoriatic Arthritis. Arthritis Rheumatol (Hoboken, NJ). 2020 Mar;72(3):435-47.

56. Xu X, Davelaar N, Mus A-M, Asmawidjaja PS, Hazes JMW, Baeten DLP, et al. IL-17A is produced by synovial fluid CD4+ but not CD8+ T cells after TCR activation and regulates different inflammatory mediators compared to TNF in a synovitis model of psoriatic arthritis. Arthritis Rheumatol. 2020 Apr 3;

57. Watad A, Rowe H, Russell T, Zhou Q, Anderson LK, Khan A, et al. Normal human enthesis harbours conventional CD4+ and CD8+ T cells with regulatory features and inducible IL-17A and TNF expression. Ann Rheum Dis. 2020 Aug;79(8):1044-54.

58. Al-Mossawi MH, Chen L, Fang H, Ridley A, de Wit J, Yager N, et al. Unique transcriptome signatures and GM-CSF expression in lymphocytes from patients with spondyloarthritis. Nat Commun. 2017 Dec 15;8(1):1510.

59. Wade SM, Canavan M, McGarry T, Low C, Wade SC, Mullan RH, et al. Association of synovial tissue polyfunctional T-cells with DAPSA in psoriatic arthritis. Ann Rheum Dis. 2019 Mar;78(3):350-4.

60. Curran SA, FitzGerald OM, Costello PJ, Selby JM, Kane DJ, Bresnihan B, et al. Nucleotide Sequencing of Psoriatic Arthritis Tissue before and during Methotrexate Administration Reveals a Complex Inflammatory T Cell Infiltrate with Very Few Clones Exhibiting Features That Suggest They Drive the Inflammatory Process by Recognizing Aut. J Immunol. 2004 Feb 1;172(3):1935-44. 


\section{CHAPTER 9}

61. Menon B, Gullick NJ, Walter GJ, Rajasekhar M, Garrood T, Evans HG, et al. Interleukin-17+CD8+ T Cells Are Enriched in the Joints of Patients With Psoriatic Arthritis and Correlate With Disease Activity and Joint Damage Progression. Arthritis Rheumatol. 2014 May;66(5):1272-81.

62. Ciccia F, Guggino G, Rizzo A, Saieva L, Peralta S, Giardina A, et al. Type 3 innate lymphoid cells producing IL-17 and IL-22 are expanded in the gut, in the peripheral blood, synovial fluid and bone marrow of patients with ankylosing spondylitis. Ann Rheum Dis. 2015 Sep;74(9):1739-47.

63. Blijdorp ICJ, Menegatti S, Mens LJJ, Sande MGH, Chen S, Hreggvidsdottir HS, et al. Expansion of Interleukin-22- and Granulocyte-Macrophage Colony-Stimulating Factor-Expressing, but Not Interleukin-17A-Expressing, Group 3 Innate Lymphoid Cells in the Inflamed Joints of Patients With Spondyloarthritis. Arthritis Rheumatol. 2019 Mar 15;71(3):392-402.

64. Cuthbert RJ, Fragkakis EM, Dunsmuir R, Li Z, Coles M, Marzo-Ortega H, et al. Brief Report: Group 3 Innate Lymphoid Cells in Human Enthesis. Arthritis Rheumatol. 2017 Sep;69(9):1816-22.

65. Soare A, Weber S, Maul L, Rauber S, Gheorghiu AM, Luber M, et al. Cutting Edge: Homeostasis of Innate Lymphoid Cells Is Imbalanced in Psoriatic Arthritis. J Immunol. 2018 Feb 15;200(4):1249-54.

66. Keren A, Shemer A, Ginzburg A, Ullmann Y, Schrum AG, Paus R, et al. Innate lymphoid cells 3 induce psoriasis in xenotransplanted healthy human skin. J Allergy Clin Immunol. 2018 Jul;142(1):305-308.e6.

67. Furue M, Furue K, Tsuji G, Nakahara T. Interleukin-17A and Keratinocytes in Psoriasis. Int J Mol Sci. 2020 Feb 13;21(4):1275.

68. Lowes MA, Suárez-Fariñas M, Krueger JG. Immunology of Psoriasis. Annu Rev Immunol. 2014 Mar 21;32(1):227-55.

69. Nograles KE, Zaba LC, Guttman-Yassky E, Fuentes-Duculan J, Suárez-Fariñas M, Cardinale I, et al. Th17 cytokines interleukin (IL)-17 and IL-22 modulate distinct inflammatory and keratinocyte-response pathways. Br J Dermatol. 2008 Aug;

70. Chiricozzi A, Guttman-Yassky E, Suárez-Fariñas M, Nograles KE, Tian S, Cardinale I, et al. Integrative Responses to IL-17 and TNF-a in Human Keratinocytes Account for Key Inflammatory Pathogenic Circuits in Psoriasis. J Invest Dermatol. 2011 Mar;131(3):677-87.

71. Dai H, Adamopoulos IE. Psoriatic arthritis under the influence of IFNy. Clin Immunol. 2020 Sep;218:108513.

72. Chiricozzi A, Suárez-Fariñas M, Fuentes-Duculan J, Cueto I, Li K, Tian S, et al. Increased expression of interleukin-17 pathway genes in nonlesional skin of moderate-to-severe psoriasis vulgaris. Br J Dermatol. 2016 Jan 11;174(1):136-45.

73. Matos TR, O'Malley JT, Lowry EL, Hamm D, Kirsch IR, Robins HS, et al. Clinically resolved psoriatic lesions contain psoriasis-specific IL-17-producing a $\mathrm{T}$ cell clones. J Clin Invest. 2017 Sep 25;127(11):4031-41.

74. Jiang $X$, Clark RA, Liu L, Wagers AJ, Fuhlbrigge RC, Kupper TS. Skin infection generates nonmigratory memory CD8+ TRM cells providing global skin immunity. Nature. 2012 Mar 29;483(7388):227-31.

75. Abji F, Pollock RA, Liang K, Chandran V, Gladman DD. Th17 gene expression in psoriatic arthritis synovial fluid and peripheral blood compared to osteoarthritis and cutaneous psoriasis. Clin Exp Rheumatol. 36(3):486-9. 
76. Benham H, Norris P, Goodall J, Wechalekar MD, FitzGerald O, Szentpetery A, et al. Th17 and Th22 cells in psoriatic arthritis and psoriasis. Arthritis Res Ther. 2013;15(5):R136.

77. Capsoni F, Molteni S, Raeli L, Diani M, Altomare A, Garavaglia M, et al. Differential expression of interleukin-2 by anti-CD3-stimulated peripheral blood mononuclear cells in patients with psoriatic arthritis and patients with cutaneous psoriasis. 2014;(II):385-90.

78. Raimondo A, Lembo S, Di Caprio R, Donnarumma G, Monfrecola G, Balato N, et al. Psoriatic cutaneous inflammation promotes human monocyte differentiation into active osteoclasts, facilitating bone damage. Eur J Immunol. 2017 Jun;47(6):1062-74.

79. Pollock RA, Abji F, Liang K, Chandran V, Pellett FJ, Virtanen C, et al. Gene expression differences between psoriasis patients with and without inflammatory arthritis. J Invest Dermatol. 2015 Feb;135(2):620-3.

80. Abji F, Pollock RA, Liang K, Chandran V, Gladman DD. Brief Report: CXCL10 Is a Possible Biomarker for the Development of Psoriatic Arthritis Among Patients With Psoriasis. Arthritis Rheumatol (Hoboken, NJ). 2016;68(12):2911-6.

81. Abji F, Lee K, Math M, Pollock RA, Machhar R, Cook RJ, et al. Declining levels of serum CXCL10 over time are associated with new onset of psoriatic arthritis in patients with psoriasis: A new biomarker? Br J Dermatol. 2020 Feb 9;

82. Pouw JN, Leijten EFA, Laar JM, Boes M. Revisiting B cell tolerance and autoantibodies in seropositive and seronegative autoimmune rheumatic disease (AIRD). Clin Exp Immunol. 2020 Nov 15;cei.13542.

83. Frasca L, Palazzo R, Chimenti MS, Alivernini S, Tolusso B, Bui L, et al. Anti-LL37 Antibodies Are Present in Psoriatic Arthritis (PsA) Patients: New Biomarkers in PsA. Front Immunol. 2018;9:1936.

84. Yuan Y, Qiu J, Lin Z-T, Li W, Haley C, Mui UN, et al. Identification of Novel Autoantibodies Associated With Psoriatic Arthritis. Arthritis Rheumatol (Hoboken, NJ). 2019 Jun;71(6):941-51.

85. Sherlock JP, Joyce-Shaikh B, Turner SP, Chao C-C, Sathe M, Grein J, et al. IL-23 induces spondyloarthropathy by acting on ROR-yt+ CD3+CD4-CD8- entheseal resident $\mathrm{T}$ cells. Nat Med. 2012 Jul;18(7):1069-76.

86. Chen L, Deshpande M, Grisotto M, Smaldini P, Garcia R, He Z, et al. Skin expression of IL23 drives the development of psoriasis and psoriatic arthritis in mice. Sci Rep. 2020 Dec 19;10(1):8259.

87. Dudakov JA, Hanash AM, van den Brink MRM. Interleukin-22: Immunobiology and Pathology. Annu Rev Immunol. 2015 Mar 21;33(1):747-85.

88. Sakkas LI, Bogdanos DP. Are psoriasis and psoriatic arthritis the same disease? The IL-23/IL17 axis data. Autoimmun Rev. 2017 Jan;16(1):10-5.

89. Reich K, Krüger K, Mössner R, Augustin M. Epidemiology and clinical pattern of psoriatic arthritis in Germany: a prospective interdisciplinary epidemiological study of 1511 patients with plaque-type psoriasis. Br J Dermatol. 2009 May;160(5):1040-7.

90. Villani AP, Rouzaud M, Sevrain M, Barnetche T, Paul C, Richard M-A, et al. Prevalence of undiagnosed psoriatic arthritis among psoriasis patients: Systematic review and metaanalysis. J Am Acad Dermatol. 2015 Aug;73(2):242-8. 


\section{CHAPTER 9}

91. Coates LC, Aslam T, Al Balushi F, Burden a D, Burden-Teh E, Burden-The E, et al. Comparison of three screening tools to detect psoriatic arthritis in patients with psoriasis (CONTEST study). Br J Dermatol. 2013 Apr;168(4):802-7.

92. Coates LC, Savage L, Waxman R, Moverley AR, Worthington S, Helliwell PS. Comparison of screening questionnaires to identify psoriatic arthritis in a primary-care population: a crosssectional study. Br J Dermatol. 2016 Sep;175(3):542-8.

93. Mease PJ, Palmer JB, Hur P, Strober BE, Lebwohl M, Karki C, et al. Utilization of the validated Psoriasis Epidemiology Screening Tool to identify signs and symptoms of psoriatic arthritis among those with psoriasis: a cross-sectional analysis from the US-based Corrona Psoriasis Registry. J Eur Acad Dermatology Venereol. 2019 May 5;33(5):886-92.

94. Karreman MC, Weel AEAM, van der Ven M, Vis M, Tchetverikov I, Nijsten TEC, et al. Prevalence of Psoriatic Arthritis in Primary Care Patients With Psoriasis. Arthritis Rheumatol. 2016 Apr;68(4):924-31.

95. Karreman MC, Weel AEAM, van der Ven $M$, Vis $M$, Tchetverikov I, Nijsten TEC, et al. Performance of screening tools for psoriatic arthritis: a cross-sectional study in primary care. Rheumatology. 2016 Dec 24;kew410.

96. Haroon M, Gallagher P, Fitzgerald O. Diagnostic delay of more than 6 months contributes to poor radiographic and functional outcome in psoriatic arthritis. 2014;1-6.

97. Coates LC, Mahmood F, Freeston J, Emery P, Conaghan PG, Helliwell PS. Long-term followup of patients in the TIght COntrol of inflammation in early Psoriatic Arthritis (TICOPA) trial. Rheumatology. 2020 Apr 1;59(4):807-10.

98. Coates LC, Moverley AR, McParland L, Brown S, Navarro-Coy N, O'Dwyer JL, et al. Effect of tight control of inflammation in early psoriatic arthritis (TICOPA): a UK multicentre, openlabel, randomised controlled trial. Lancet. 2015 Dec;386(10012):2489-98.

99. Iragorri N, Hazlewood G, Manns B, Danthurebandara V, Spackman E. Psoriatic arthritis screening: a systematic review and meta-analysis. Rheumatology. 2019 Apr 1;58(4):692-707.

100. Boutet M-A, Nerviani A, Gallo Afflitto G, Pitzalis C. Role of the IL-23/IL-17 Axis in Psoriasis and Psoriatic Arthritis: The Clinical Importance of Its Divergence in Skin and Joints. Int J Mol Sci. 2018 Feb 9;19(2):530.

101. Gossec L, Baraliakos X, Kerschbaumer A, de Wit M, McInnes I, Dougados M, et al. EULAR recommendations for the management of psoriatic arthritis with pharmacological therapies: 2019 update. Ann Rheum Dis. 2020 Jun 20;79(6):700-12.

102. Ogdie A, Coates LC, Gladman DD. Treatment guidelines in psoriatic arthritis. Rheumatology. 2020 Mar 1;59(Supplement_1):i37-46.

103. Coates LC, Kavanaugh A, Mease PJ, Soriano ER, Laura Acosta-Felquer M, Armstrong AW, et al. Group for Research and Assessment of Psoriasis and Psoriatic Arthritis 2015 Treatment Recommendations for Psoriatic Arthritis. Arthritis Rheumatol. 2016 Mar;n/a-n/a.

104. Litman T. Personalized medicine-concepts, technologies, and applications in inflammatory skin diseases. APMIS. 2019 May 24;127(5):386-424.

105. Nagy G, Roodenrijs NM, Welsing PM, Kedves M, Hamar A, van der Goes MC, et al. EULAR definition of difficult-to-treat rheumatoid arthritis. Ann Rheum Dis. 2021 Jan;80(1):31-5. 
106. Roodenrijs NMT, de Hair MJH, van der Goes MC, Jacobs JWG, Welsing PMJ, van der Heijde D, et al. Characteristics of difficult-to-treat rheumatoid arthritis: results of an international survey. Ann Rheum Dis. 2018 Dec;77(12):1705-9.

107. Leijten EFA. A precision medicine trial in psoriatic arthritis-first jump in the deep end. Rheumatology (Oxford). 2019 Feb 1;58(2):189-90.

108. Miyagawa I, Nakayamada S, Nakano K, Kubo S, Iwata S, Miyazaki Y, et al. Precision medicine using different biological DMARDs based on characteristic phenotypes of peripheral T helper cells in psoriatic arthritis. Rheumatology. 2019 Feb 1;58(2):336-44.

109. Teitsma XM, Jacobs JWG, de Jong PHP, Hazes JMW, Weel AEAM, Welsing PMJ, et al. Adding baseline protein biomarkers to clinical predictors does not enhance prediction of treatment response to a methotrexate strategy in early rheumatoid arthritis. Ann Rheum Dis. 2019 Jan;78(1):142-4.

110. Tomalin LE, Kim J, Correa da Rosa J, Lee J, Fitz LJ, Berstein G, et al. Early Quantification of Systemic Inflammatory Proteins Predicts Long-Term Treatment Response to Tofacitinib and Etanercept. J Invest Dermatol. 2020 May;140(5):1026-34.

111. Brodmerkel C, Li K, Garcet S, Hayden K, Chiricozzi A, Novitskaya I, et al. Modulation of inflammatory gene transcripts in psoriasis vulgaris: Differences between ustekinumab and etanercept. J Allergy Clin Immunol. 2019 May;143(5):1965-9.

112. Visvanathan S, Baum P, Vinisko R, Schmid R, Flack M, Lalovic B, et al. Psoriatic skin molecular and histopathologic profiles after treatment with risankizumab versus ustekinumab. J Allergy Clin Immunol. 2019 Jun;143(6):2158-69.

113. Steel KJA, Srenathan U, Ridley M, Durham LE, Wu S, Ryan SE, et al. Polyfunctional, Proinflammatory, Tissue-Resident Memory Phenotype and Function of Synovial Interleukin$17 \mathrm{~A}+\langle\mathrm{scp}\rangle \mathrm{CD}</ \mathrm{scp}\rangle$ 8+ T Cells in Psoriatic Arthritis. Arthritis Rheumatol. $2020 \mathrm{Mar}$ 4;72(3):435-47.

114. Belasco J, Louie JS, Gulati N, Wei N, Nograles K, Fuentes-Duculan J, et al. Comparative genomic profiling of synovium versus skin lesions in psoriatic arthritis. Arthritis Rheumatol. 2015;67(4):934-44.

115. Chen S, Blijdorp IC, van Mens LJJ, Bowcutt R, Latuhihin TE, van de Sande MGH, et al. Interleukin 17A and IL-17F Expression and Functional Responses in Rheumatoid Arthritis and Peripheral Spondyloarthritis. J Rheumatol. 2020 Nov 1;47(11):1606-13.

116. Nerviani A, Boutet M-A, Tan WSG, Goldmann K, Purkayastha N, Lajtos TA, et al. IL-23 skin and joint profiling in psoriatic arthritis: novel perspectives in understanding clinical responses to IL-23 inhibitors. Ann Rheum Dis. 2020 Nov 26;annrheumdis-2020-218186.

117. Aydin SZ, Bridgewood C, Zabotti A, Girolimetto N, McGonagle D. The transition from enthesis physiological responses in health to aberrant responses that underpin spondyloarthritis mechanisms. Curr Opin Rheumatol. 2020 Nov 20;33(1):64-73. 


\section{LIST OF PUBLICATIONS}

1. Leijten EFA, van Kempen TS, Boes M, Michels-van Amelsfort JMR, Hijnen D, Hartgring SAY, et al. Brief Report: Enrichment of Activated Group 3 Innate Lymphoid Cells in Psoriatic Arthritis Synovial Fluid. Arthritis Rheumatol. 2015 Oct;67(10):2673-8.

2. van Kempen TS, Wenink MH, Leijten EFA, Radstake TRDJ, Boes M. Perception of self: distinguishing autoimmunity from autoinflammation. Nat Rev Rheumatol. 2015 Aug 12;11(8):483-92.

3. Wenink MH, Leijten EFA, Cupedo T, Radstake TRDJ. Review: Innate Lymphoid Cells: Sparking Inflammatory Rheumatic Disease? Arthritis Rheumatol (Hoboken, NJ). 2017 May 26;69(5): 885-97.

4. Leijten EFA, Sigurdsson V, Wenink MH, Radstake TRDJ. Screening for psoriatic arthritis using the Psoriasis Epidemiology Screening Tool questionnaire: examining the optimal cut-off. $\mathrm{Br} J$ Dermatol. 2017 May;176(5):1357-9.

5. Affandi AJ, Silva-Cardoso SC, Garcia S, Leijten EFA, van Kempen TS, Marut W, et al. CXCL4 is a novel inducer of human Th17 cells and correlates with IL-17 and IL-22 in psoriatic arthritis. Eur J Immunol. 2018 Mar;48(3):522-31.

6. Leijten EFA, Radstake TRDJ, Reedquist KA. Editorial: Lessons Learned From a "Failed" Clinical Trial. Arthritis Rheumatol (Hoboken, NJ). 2018;70(9):1364-5.

7. Verhagen FH, Hiddingh S, Rijken R, Pandit A, Leijten E, Olde Nordkamp M, et al. HighDimensional Profiling Reveals Heterogeneity of the Th17 Subset and Its Association With Systemic Immunomodulatory Treatment in Non-infectious Uveitis. Front Immunol. 2018 Oct 31;9:2519.

8. Pouw JN, Leijten EFA, Tekstra J, Balak DMW, Radstake TRDJ. [Spectrum of psoriatic conditions]. Ned Tijdschr Geneeskd. 2019 Jul 29;163.

9. Blokland SLM, van den Hoogen LL, Leijten EFA, Hartgring SAY, Fritsch R, Kruize AA, et al. Increased expression of Fas on group 2 and 3 innate lymphoid cells is associated with an interferon signature in systemic lupus erythematosus and Sjögren's syndrome. Rheumatology (Oxford). 2019 Apr 8;

10. Leijten EFA. A precision medicine trial in psoriatic arthritis-first jump in the deep end. Rheumatology (Oxford). 2019;58(2):189-90.

11. Leijten EF, Radstake TR, McInnes IB, Jacobs JW. Limits of traditional evidence-based medicine methodologies exemplified by the novel era in psoriatic arthritis drug development. Expert Rev Clin Immunol. 2019 May 4;15(5):441-4.

12. Laban KG, Kalmann R, Bekker CPJ, Hiddingh S, van der Veen RLP, Eenhorst CAE, Genders S, Mourtis M, Verhagen F, Leijten E, Haitke, a S, de Groot M, et al. A pan-inflammatory microRNAcluster is associated with orbital non-Hodgkin lymphoma and idiopathic orbital inflammation. Eur J Immunol. 2020 Jan;50(1):86-96. 
13. van Kempen TS, Leijten EFA, Lindenbergh MFS, Nordkamp MO, Driessen C, Lebbink R-J, et al. Impaired proteolysis by SPPL2a causes CD74 fragment accumulation that can be recognized by anti-CD74 autoantibodies in human ankylosing spondylitis. Eur J Immunol. 2020 Mar 21;

14. Pouw J, Leijten E, Radstake T, Boes M. Emerging molecular biomarkers for predicting therapy response in psoriatic arthritis: A review of literature. Clin Immunol. 2020 Feb;211:108318.

15. Pouw JN, Leijten EFA, Laar JM, Boes M. Revisiting B cell tolerance and autoantibodies in seropositive and seronegative autoimmune rheumatic disease (AIRD). Clin Exp Immunol. 2020 Nov 15;cei.13542.

16. Kleinrensink NJ, Foppen W, Ten Katen I, van der Veen PH, de Klerk B, Diepstraten SCE, Radstake T, Lafeber F, De Jong P, Leijten E. Comparison of the Heel Enthesitis MRI Scoring System (HEMRIS) with clinical enthesitis and local metabolic activity on PET-CT. RMD open. 2020;6(3).

17. Jacobs ME, Pouw JN, Welsing P, Radstake TRDJ, Leijten EFA. First-line csDMARD monotherapy drug retention in psoriatic arthritis: methotrexate outperforms sulfasalazine. Rheumatology (Oxford). 2020 Aug 14;

20. Leijten E, Tao W, Pouw J, van Kempen T, Olde Nordkamp M, Balak D, et al. Broad proteomic screen reveals shared serum proteomic signature in patients with psoriatic arthritis and psoriasis without arthritis. Rheumatology (Oxford). 2020 Aug 13;

19. Jacobs ME, Pouw JN, Olde Nordkamp MA, Radstake TRDJ, Leijten EFA, Boes M. DNAM1 and TIGIT balance the T cell response, with low T cell TIGIT expression corresponding to inflammation in psoriatic disease. Immunother Adv. 2021 Jan 1;1(1)

20. Leijten EF, van Kempen TS, Olde Nordkamp MA, Pouw JN, Kleinrensink NJ, Vincken NL, et al. Tissue-resident memory CD8+ T cells from skin differentiate psoriatic arthritis from psoriasis. Arthritis Rheumatol (Hoboken, NJ). 2021 Jan 16; 


\section{NEDERLANDSE SAMENVATTING}

\section{Psoriasis en artritis psoriatica: voorbij de dichotomie}

De belangrijkste onderzoeksvragen van dit proefschrift zijn:

- Hoe kunnen we screenen op artritis psoriatica en hoe verloopt de behandeling van artritis psoriatica in de dagelijkse praktijk? (Hoofdstuk 2-3)

- Waarom ontwikkelen sommige patiënten met psoriasis wel artritis psoriatica, en andere niet? (Hoofdstuk 4-7)

- Wat zijn de belangrijkste uitdagingen voor toekomstig onderzoek naar psoriasis en artritis psoriatica? (Hoofdstuk 8)

Psoriasis is een auto-immuunaandoening die gepaard gaat met schilferende, rode, jeukende plekken op de huid. Veelvoorkomende lokaties zijn de strekzijde van de ellebogen, de knieën, de genitaliën en de behaarde hoofdhuid. Soms zijn er ook afwijkingen aan de nagels. Psoriasis komt relatief vaak voor: bij zo'n twee procent van bevolking. Een aanzienlijk deel van de psoriasispatiënten ontwikkelt ook reumatische klachten. Als een patiënt met psoriasis ook gewrichtsontstekingen (artritis) ontwikkelt kan er sprake zijn van artritis psoriatica (APs). De reumatische uitingen van APs beperken zich niet tot artritis. Ook ontstekingen van peesaanhechtingen (enthesitis), een ontsteking van een gehele vinger of teen (dactylitis) en ontsteking van het bekkengewricht (sacro-iliitis) worden soms gezien. APs heeft overeenkomsten met spondylitis ankylopoetica (voorheen: ziekte van Bechterew). Er is geen duidelijke relatie tussen de ernst van de psoriasis en de ernst van de reumatische klachten. Sommige patiënten hebben zeer ernstige psoriasis maar krijgen nooit last van hun gewrichten. Er zijn ook mensen die nauwelijks psoriasis hebben maar juist wel veel reumatische klachten. Soms reageert de psoriasis goed op een behandeling maar de artritis minder goed. Zowel psoriasis als APs kunnen een nadelig effect hebben op de kwaliteit van leven.

Een belangrijke vraag die dit proefschrift tracht te beantwoorden is: waarom ontwikkelen sommige patiënten met psoriasis wel APs, en andere niet? Als we beter begrijpen welke biologische processen aan de ziekte ten grondslag liggen, kunnen we in de toekomst ook een betere behandeling bieden. Onze insteek was: als we deze twee groepen vergelijken, kunnen we dan ontdekken of er specifieke ziekteprocessen gaande zijn in APs? 
In de introductie laten we zien dat het al langer bekend is dat psoriasis vaker dan je op basis van toeval zou verwachten gepaard gaat met reumatische klachten. Halverwege de twintigste eeuw werd steeds duidelijker dat APs een ander ziektebeeld is dan de klassieke vorm van reuma (reumatoïde artritis (RA)). Deze inzichten zijn deels te danken aan ontwikkelingen op het gebied van de genetica en deels aan klinisch onderzoek. Sommige specifieke ziektebeelden (onder andere uveïtis en colitis) bleken namelijk veel vaker voor te komen bij patiënten met psoriasis. Waar mensen met APs aan het begin van de eenentwintigste eeuw nog zo goed als dezelfde behandeling kregen als mensen met RA, was bij de start van dit promotieonderzoek al wel bekend dat de onderliggende oorzaak verschillend was. Dit proefschrift verdiept zich verder in de verschillen tussen psoriasis en APs. Hoe vaak komt APs voor bij patiënten met psoriasis? In hoofdstuk twee van dit proefschrift staat die vraag centraal. In samenwerking met de afdeling Dermatologie werden patiënten met psoriasis, ongeacht de aan- of afwezigheid van gewrichtsklachten, uitgenodigd voor een beoordeling op de polikliniek reumatologie. Twintig tot dertig procent van de psoriasispatiënten bleek ook APs te hebben. Opvallend was dat de ernst van de psoriasis bij patiënten met en zonder APs vergelijkbaar was. Ook de ontstekingswaarden in het bloed verschilden niet noemenswaardig. We keken in hoeverre een in het buitenland al gangbare, maar in Nederland nog weinig gebruikte vragenlijst toegepast kon worden om patiënten met APs te identificeren. Het bleek dat het gebruik van deze vragenlijst ("Psoriasis Epidemiology Screening Tool") de dermatoloog kan helpen te bepalen bij welke psoriasispatiënt een verwijzing naar de reumatoloog zinvol is. Deze vragenlijst wordt in het UMC Utrecht nu standaard gebruikt: een mooi voorbeeld van op de patiënt afgestemde, wetenschappelijk gefundeerde samenwerking tussen verschillende disciplines binnen het ziekenhuis.

De effecten van frequentere en eerdere verwijzingen naar de reumatoloog zullen nog moeten worden onderzocht. Wellicht helpt deze nieuwe praktijk schade aan gewrichten te voorkomen. Ook het effect op de kwaliteit van leven van patiënten en kosteneffectiviteit moeten verder in kaart worden gebracht.

Een belangrijke stap in de behandeling van APs bestaat uit het starten van conventional disease-modifying antirheumatic drugs (cDMARDs). Deze behandelstrategie is deels gebaseerd op de wetenschap dat cDMARDs een belangrijke bijdrage hebben geleverd aan het voorkómen van gewrichtsschade bij patiënten met RA. Maar hoe ziet de behandeling van een patiënt met APs er in de dagelijkse praktijk uit? Dat wordt beschreven in hoofdstuk drie. We keken hoe lang een eerste CDMARD als monotherapie wordt voorgeschreven. Uit dit onderzoek bleek dat patiënten met APs hun eerste cDMARD gemiddeld ruim tweeënhalf jaar als monotherapie gebruiken. Binnen de groep cDMARDs werd methotrexaat gemiddeld langduriger voorgeschreven dan sulfasalazine. 
Deze bevindingen ondersteunen de aanbeveling om methotrexaat voor te schrijven voor de behandeling van artritis bij APs. Bovendien onderstreept onze studie het feit dat patiënten niet alleen stoppen omdat een geneesmiddel ineffectief is: een groot deel van de patiënten stopt vanwege bijwerkingen. Deze kennis moet worden meegenomen in toekomstig onderzoek naar een behandeling op maat. We zullen niet alleen de processen moeten bestuderen die voorspellen of een geneesmiddel effectief is op het biologische niveau, maar ook andere factoren, zoals bijwerkingen, die bepalen of een geneesmiddel voor de individuele patiënt goed werkt.

Psoriasis en APs zijn aandoeningen van het immuunsysteem. In hoofdstuk vier en vijf hebben we gericht onderzoek gedaan naar één soort immuuncel: de innate lymphoid cell (ILC). In tegenstelling tot de meest bekende en veelvoorkomende witte bloedcel, de T-lymfocyt, horen de veel zeldzamere ILCs bij het aangeboren afweersysteem. Omdat ILCs, anders dan de T-lymfocyt, geen specifieke indringers hoeven te herkennen kunnen ze relatief snel zorgen voor een afweerreactie. Ondanks deze verschillen kunnen ILCs cytokines (ontstekingseiwitten) produceren die ook door T-lymfocyten worden gemaakt: bijvoorbeeld tumor-necrose-factoralfa en interleukine-17. De laatste jaren zijn verschillende nieuwe soorten ILCS ontdekt. Vaak is van die cellen nog onbekend waar ze zich in het lichaam ophouden en bij welke ziektebeelden ze een rol spelen. Het is duidelijk dat ILCs aanwezig zijn in de huid en daar een bijdrage kunnen leveren aan het ontstaan van psoriasis. We kwamen er achter dat ILCs óók aanwezig zijn in gewrichtsvloeistof en dat een bepaald type ILC (ILC3) ook nog eens vaker voorkomt in de gewrichtsvloeistof van patiënten met APs. Deze bevindingen ondersteunen de hypothese dat specifieke cellen van het aangeboren afweersysteem een belangrijke rol kunnen spelen in het ontstaan van APs bij psoriasispatiënten.

Voor de volgende hoofdstukken vergeleken we immuuncellen (hoofdstuk zes) en eiwitten (hoofdstuk zeven) uit het bloed van patiënten met psoriasis en APs. In hoofdstuk zes hebben we een breed palet aan immuuncellen in het bloed vergeleken en kwamen we tot de ontdekking dat de afweercellen van patiënten met psoriasis en APs vergelijkbaar zijn. Het enige verschil tussen psoriasis en APs was dat een bepaalde T lymfocyt (CCR10+ CD8 T-lymfocyt) bij APs net iets vaker bleek voor te komen. De eerste gedachte was dat deze T-lymfocyt simpelweg een slechte cel was die de gewrichtsklachten van APs veroorzaakt. Maar uit ons onderzoek blijkt, dat deze cel niet voorkomt in de gewrichtsvloeistof maar vooral terug te vinden is in de huid. De cel was daarnaast juist in staat om ontstekingsprocessen te dempen. De vraag dringt zich op: is de CCR10+ CD8 T-lymfocyt onderdeel van een compensatiemechanisme in de huid dat het onderliggende ziekteproces van APs probeert tegen te gaan? Hoofdstuk zeven laat zien hoe we met een nieuwe techniek (Olink Platform) zo'n duizend eiwitten in heel kleine hoeveelheden bloed hebben gemeten. Opnieuw vergeleken we psoriasispatienten met en zonder APs. 
En wat bleek? Beide patiëntengroepen hadden een zeer vergelijkbaar eiwitprofiel. De kennis opgedaan uit deze twee hoofdstukken geeft twee inzichten. Ten eerste betekent het dat we op korte termijn nog geen simpele diagnostische bloedtest kunnen ontwikkelen om patiënten met (een vroege fase van) APs te identificeren binnen de grotere populatie van psoriasispatiënten. Onze onderzoeken sluiten niet uit dat er op weefselniveau wel verschillen zijn. Ten tweede zullen we de pathogenese van de ziekte op cel- en zelfs moleculair niveau in een andere richting moeten onderzoeken: in de aangedane weefsels, zoals huid en gewrichten.

In hoofdstuk acht bespreken we de ontwikkeling van nieuwe geneesmiddelen bij psoriasis en APs. Er zijn de laatste jaren veel nieuwe medicijnen ontwikkeld en waarschijnlijk komen er in de nabije toekomst nog meer op de markt. Behandelaars krijgen zo steeds meer mogelijkheden om de patiënt een passende, gepersonaliseerde therapie te bieden. Maar welke van de oudere of nieuwere geneesmiddelen kunnen we nu het beste voorschrijven en in welke volgorde? De klassieke geneesmiddelenstudie is een gerandomiseerd, dubbelblind placebogecontroleerd onderzoek. Deze opzet is echter minder geschikt om bovenstaande vraag te beantwoorden: APs is heterogeen én er zijn inmiddels teveel geneesmiddelen op de markt om ze stuk voor stuk met elkaar te vergelijken. Een van de oplossingen die we voorstellen is om altijd één geneesmiddel als maatstaf te nemen, als je nieuwe middelen in een vergelijkend onderzoek opneemt. Daarnaast is het belangrijk om de ruwe data van deze studies openbaar te stellen, zodat op een later tijdstip de geneesmiddelen uit verschillende onderzoeken beter met elkaar kunnen worden vergeleken. Daarnaast zal er meer moeten worden geïnvesteerd in het ontwikkelen en uitvoeren van studies met een innovatieve opzet, meer gericht op gepersonaliseerde zorg.

De discussie van dit proefschrift beschrijft de pathogenese van psoriasis en APs volgens de laatste inzichten. In de discussie wordt ook gesteld dat deze twee ziektebeelden minder dichotoom zijn dan bij aanvang van dit proefschrift werd verondersteld. Het tweede deel van de discussie sluit af met aanbevelingen voor toekomstig onderzoek om openstaande vragen te beantwoorden.

Daarbij moet onze aandacht gericht blijven op degene om wie wetenschappelijk onderzoek in de geneeskunde draait: de patiënt. Patiënten, artsen en nietklinisch werkende wetenschappers moeten actief samenwerken, zeker als het laboratoriumonderzoek betreft. Daarbij is tijd één van de belangrijkste voorwaarden voor succesvol wetenschappelijk onderzoek. We moeten durven investeren in doelen op de lange termijn. 


\section{DANKWOORD}

Ten eerste wil ik alle patiënten bedanken die hebben deelgenomen aan het onderzoek. Door jullie bijdrage komt een betere behandeling voor deze aandoeningen stapje voor stapje dichterbij. Bedankt voor jullie geduld en blijvende inzet.

Professor Radstake, beste Tim ik kan me heel goed herinneren dat professor Bijlsma me aan jou heeft geïntroduceerd. Ik mocht een keertje met je afspreken, je zat toen tijdelijk op een kamertje op D2 west en de afspraak verliep zoals die in de toekomst vaker zou verlopen: wat hectisch, ik snapte niet zo goed waar het onderzoek over zou gaan, maar je was superenthousiast en blij om mij als onderdeel van je PsA team te hebben. In de loop der jaren heb je me echt altijd ondersteund en tegelijkertijd flink uitgedaagd om heel veel nieuwe dingen te leren, die bij het inhoudelijke onderzoek horen maar ook bij alles wat daar overstijgend bij komt kijken. Ik kan je rebelse karakter waarderen en heb vaak genoten van je gemopper als er weer stoeiwerk was met de bureaucratische aspecten van onderzoek. Je hebt me laten zien dat je buiten de hokjes moet durven denken. Veel dank voor alles, deze tijd zal me altijd bijblijven.

Dr. Boes, beste Marianne ik zie je eigenlijk al jaren éénmaal per week voor onze onderzoeksmeeting. De meeting heeft diverse vormen gehad, met meer of minder mensen, en het blijft tot op de dag van vandaag een enorme eer om telkens van je te mogen leren. Ik heb de basisprincipes van het wetenschappelijke onderzoek van jou geleerd. De kern die me zal bijblijven (en die Tim ook met je deelt) is dat onderzoek doen alleen kan met een positieve insteek: het glas is half vol, anders doe je geen onderzoek. Met al je ervaring en expertise ben je nog steeds nieuwsgierig en weet je iedereen te enthousiasmeren voor het meest simpele experiment en zelfs als het experiment niet lukt. Belangrijker nog: je weet iedereen gerust te stellen als het even tegen zit. Dan zeg je: dat hoort er echt bij en het komt ook altijd goed. En dat klopt, dank. Samen met Tim vormde je naadloos één team al deze jaren. Een beter duo had ik niet kunnen wensen.

De basis van het PsA onderzoek in het UMC Utrecht bestond uit een relatief klein groepje mensen. Samen met Tessa en Michel hebben we enorm veel patiëntmateriaal opgewerkt gedurende meerdere jaren en altijd met een gemoedelijke sfeer. Dit proefschrift is voor een groot deel ook te danken aan jou Tessa, mijn lab buddy. Ik heb je tekening van een enthesis een blijvende plek gegeven in het figuur van de discussie. Michel ook jij hebt een enorm belangrijke rol gehad in mijn promotietraject. Je hebt veel rust, relativeringsvermogen en humor. We waren in de jaren dat Tessa ons had achtergelaten dan ook een hecht team. De enige uitdaging die we aan ons voorbij hebben laten gaan was het single- 
cel sorting in het WKZ: er zaten daar op een gegeven moment zoveel bovenaardse krachten in de weg (elektromagnetische velden, toe maar) dat we het toen hebben opgegeven. Mark, jij stond aan de basis van mijn promotietraject, en dat terwijl ik met blanco kennis het lab op kwam. Desondanks heb je me geholpen om niet al te lang daarna het ILC artikel te schrijven, waarbij ik zelf pas veel later tot de ontdekking kwam dat het een zeer belangrijke publicatie was voor het vakgebied. Ik heb veel respect voor je kennis en nieuwsgierigheid, waardoor je me altijd bleef uitdagen om de verdieping te zoeken. Veel dank en ik verheug me op de mogelijkheden om in de toekomst mooi onderzoek samen te kunnen doen.

In de loop der jaren is het PsA team steeds groter geworden, nu met Nanette, Juliette, Nienke, en Rianne in de basis. Dank dat jullie het zo enthousiast hebben overgenomen. Marleen bedankt voor de fijne samenwerking op het cSDMARD manuscript, het was heel plezierig jou te mogen begeleiden. Jingwen thank you for the nice collaboration. Juliette en Nienke dank voor jullie enthousiasme en welwillendheid om me altijd te helpen, ik ben vereerd jullie te mogen begeleiden. Een essentieel onderdeel van het PsA onderzoeksteam zijn de research nurses. Ik heb ongelofelijk veel respect voor hoe positief en vriendelijk jullie het dagelijkse werk doen. Patiënten zijn altijd positief over jullie waardoor ik met vol vertrouwen met jullie heb samengewerkt de laatste jaren. Dat maakt het als onderzoeker ontzettend fijn en motiverend. Heel erg veel dank Karin, Anneloes en Joke.

\begin{abstract}
Alsya it was nothing less than fate that I got the magic desk right next to yours. I was thankful for that seat every day I came to the office. You were and are ever calm, friendly and willing to drop everything to help others (beware: elk voordeel heb z'n nadeel). The work in this thesis does not have your name on it as co-author, but all aspects have your signature in there. Thank you so much. Luuk de beste wetenschappelijke discussies die ik tijdens mijn promotietijd voerde waren met jou tijdens de fietstocht naar huis. Je bent een gedisciplineerde en getalenteerde translationele onderzoeker en ik heb veel van je geleerd, dank.
\end{abstract}

Aridaman and Deepak you both know why I mention you together. What an adventure. From the airport straight to chicken, spinning tables designed for respectful interaction between guest and an ever-increasing number of bosses, big bosses, super bosses and super big bosses. Aridaman thanks for all your help on the work in this thesis and for your beautifully critical perspective, I look forward to continued collaboration. Deepak enorm bedankt voor je vriendelijkheid en betrokkenheid bij het delen van je dermatologische expertise met ons, hopelijk blijf je die delen (ik weet je te vinden). Sarita bedankt voor je humor en dat ik je altijd om advies mocht vragen als ik ergens mee vast liep, ik heb altijd veel vertrouwen gehad in je brede kennis en kunde. Chiara thanks for the wonderful collaboration we always had and hopefully we can continue working together at some point in 
the future; you are a very talented researcher and I am always honoured to work with you. Jonas our collaboration has been deemed by some (though not all) as the best collaboration in history for organizing the Radstake retreat in summerstyle tents in the cold and windy Ardennes. Jonas bedankt voor de kennis die je wist te delen en de grote lijnen die je overziet. Ik heb veel respect voor het feit dat je als basale wetenschapper ook vloeiend de taal van de arts(-onderzoeker) spreekt. Joel bedankt voor de altijd plezierige samenwerking. Kris bedankt voor de fijne samenwerking, ik heb veel van je geleerd. Floris bedankt voor je adviezen en de rol die je op je hebt genomen voor mij na het vertrek van Tim. Erik bedankt voor de fijne samenwerking op het CCR10 artikel, hopelijk overlappen onze paden vaker in de toekomst. Sam and Ernesto thank you for the pleasant collaboration and for sharing your scientific expertise with me.

Anne Karien ontzettend bedankt voor je hulp en betrokkenheid bij alle projecten. Zonder jou heerlijk nuchtere, soms strenge maar vooral pragmatische blik was dit onderzoek niet mogelijk geweest. Paco bedankt voor je bereidheid om me altijd te helpen, soms informeel en soms formeel. Ik waardeer dat je altijd open staat om je kennis te delen: niet zomaar een analyse uitvoeren, maar je wil het in alle rust uitleggen, stapsgewijs, zodat ik ook begrijp waarom we bepaalde keuzes maken. Hierdoor creëerde je altijd het gevoel dat we samen een onderdeel waren van één team, veel dank.

Professor Mclnnes, dear lain thank you for your enthusiasm on the concepts presented in the manuscript from Chapter 8, even when we ended up having to remove the quote from Johan Cruyff.

Bedankt Cornelis (micro-RNA isoleren is het engste dat ik ooit heb gedaan), Bea (thanks for helping out the PsA team when we were in need), Fleurieke (bedankt voor je precisie en plezierige samenwerking), Weiyang (thanks for the great and pleasant collaboration we always have, I wish you and your family the best), Sandra (thanks for your patience and support in the lab, it was much appreciated), Ana (thanks for your expertise and help). Bedankt Jorre, Sofie, Nadia, Andrea, Nila, Kamil, Pawel, Gerdien, Kim, Eefje, Annemieke, Fréderique, Sanne, Ralph, Rina, Marlot, Anneline, Maarten Hillen, Arno, Marion, Lotte, Francesca, Marthe en alle andere colleg a's die het leven gezellig hebben gemaakt tijdens mijn promotietraject. Thanks to Tiago, Wiola, Abhi, Marzia, Samu, Barbara, Elena, Mailli, Marta, Julia for making life at the lab so much fun. Diana bedankt voor je humor en hulp met alle logistieke dingen die ik niet kon overzien. Saskia, Yvonne, en Sigrid bedankt voor jullie ondersteuning altijd. Jeroen en Pien bedank voor jullie flow-expertise. 
Ik moet specifiek ook mijn opleiders bedanken, zonder jullie ondersteuning van mijn persoonlijke ontwikkeling had ik deze mooie levenservaring niet kunnen hebben. Hans Bijlsma bedankt dat je me destijds de ruimte gaf om een promotietraject pas te starten op het moment dat ik daar zelf ook aan toe was. Jaap bedankt voor je vertrouwen in mij de laatste jaren en je wijze adviezen, die blijven me bij. Evelien bedankt voor je flexibiliteit om me de ruimte te gunnen voor het onderzoek en het afronden van mijn proefschrift.

Jocea bedankt voor de begeleiding bij de start van mijn promotietraject. Ik was heel blij dat je me met je wijsheid kon helpen om het overzicht te bewaken van alle projecten die op me afkwamen. Janneke ik heb het geluk gehad dat je me wilde superviseren toen ik ook patiëntenzorg ging doen tijdens mijn promotietraject. Je hebt me veel van het vak geleerd en bent in de loop der jaren voor mij een belangrijk rolmodel geworden. Vigfús bedankt dat je altijd open stond voor het smeden van plannen. Zomaar even een samenwerking opzetten tussen dermatologie en reumatologie? Normaliter loop je vast in administratief of bureaucratisch gedoe, maar bij jou was dat echt anders. Je had altijd een positieve en pragmatische houding waardoor het onderzoek in dit proefschrift mogelijk was. Pim en Wouter het stukje beeldvorming zit niet in dit proefschrift maar ik heb de laatste jaren onze samenwerking als erg plezierig ervaren, en tijdens die periode veel geleerd van jullie rust, wetenschappelijke nuchterheid en inhoudelijke kennis, waarvoor dank. Hans Jacobs bedankt voor je bijdrage aan het proefschrift. Dank voor je precisie, degelijkheid en structuur. Ik heb ontzettend veel van je geleerd, met als mooiste les: wees geen lemming. Marlies, Erwin, en Jasper ook jullie hebben voor mij een belangrijke voorbeeldfunctie gehad, waarvoor dank. Alle collega AIOS bedankt voor jullie aanmoedigingen, dat was echt heel fijn. Hopelijk na de zomer hebben we allemaal meer tijd en mogelijkheden om elkaar op te zoeken.

Wouter, Faas, Douwe bedankt voor het goede voorbeeld. Verheug me op meer avonturen samen nu ik het proefschrift achter de rug heb. Wijkers bedankt voor de uitjes, relativeren in een trein is altijd goed. Maarten ik verheug me er zo ontzettend op dat jij een snoekbaars gaat vangen in de rivier, en ja, met een lekker zonnetje erbij. Gabriel ik verheug me er op meer te gaan (vlieg)vissen, dat is belangrijk in het leven. Roel bedankt voor je "bijdrage" aan de Nederlandse samenvatting. Michiel bedankt voor de jaarlijkse opfrisser met zon, strand en zee. Wim bedankt voor de fijne wetenschappelijke discussies, je houdt me scherp. Hopelijk nu meer tijd voor de kinderen om samen tijd door te brengen. Pap bedankt voor de discussies, je kritische blik en het bewijs dat een vlo luistert met z'n poten. Mam bedankt voor het altijd inspringen om te helpen met de kinderen. Koos en Willy bedankt voor het zo vaak inspringen met de kinderen. Wat een fijne Opa's en Oma's hebben Bess en Idris toch. Zonder jullie bijdrage hier in Utrecht was het niet mogelijk geweest om (af en toe) hard te werken. Sabine, Ard, Fenna, Bojan, Kilian, Rosa, en Wouter: bedankt voor al het meeleven, snel weer meer samen zijn. 
Idris en Bess jullie zijn de liefste kinderen ter wereld en wat zijn jullie lief geweest voor je papa en mama, ondanks dat we zo vaak in de avonden moesten werken aan ons proefschrift. Willemijn ik hou van je-het was niet gelukt zonder je geduld maar vooral liefde-dank je wel, dit proefschrift is ook van jou. En nu is het proefschrift eindelijk klaar! 


\section{CURRICULUM VITAE}

Emmerik Leijten was born $19^{\text {th }}$ of January 1984 in Miami, Florida. He went to elementary school in the Netherlands and attended high school in New Jersey, the United States. In 2002 he moved to Groningen to study Medicine at the Rijksuniversiteit Groningen and performed the last two years of his internships in Deventer. After finishing Medical School in 2008, he took courses on Medical Anthropology and Sociology at the University of Edinburgh in 2009 and worked as clinician on the Internal Medicine ward of the Diakonessenhuis in Paramaribo, Suriname in 2010. Afterwards he starting his training to become a rheumatologist at the UMC Utrecht, with training for Internal Medicine at the Diakonessenhuis in Utrecht. In 2013 he started his PhD at the Laboratory of Translational Medicine in the group of prof. dr. Radstake and dr. Boes. He performed translational research on psoriasis and psoriatic arthritis, striving to uncover clinical and immunological questions. He enjoys teaching medical students, with specific enthusiasm for the field of rheumatology. He has been an invited speaker at congresses, is member of the editorial board of a scientific journal and helps supervise medical students and PhD students in the research field of psoriatic arthritis. After leaving the lab, he continued training in the St. Antonius hospital and is now at the UMC Utrecht, planning to become a rheumatologist in the summer of 2021. Outside of work Emmerik very much enjoys football and (fly)fishing, but is happiest simply at home in Utrecht with Willemijn and their two lovely children Bess and Idris. 

